\title{
Aprofundando as noções de dependência e envelhecimento em distribuições de probabilidade bivariadas
}

Jayme Augusto Duarte Pereira Pinto

\author{
TESE APRESENTADA \\ $\mathrm{AO}$ \\ Instituto DE MATEMÁticA E EstatísticA \\ DA \\ Universidade DE SÃo PAUlo \\ PARA \\ OBTENÇÃO DO TÍTULO \\ $\mathrm{DE}$ \\ Doutor EM CiÊnCIAS
}

\begin{abstract}
Programa: Estatística
Orientador: Nikolai Kolev

Durante o desenvolvimento deste trabalho o autor recebeu auxílio financeiro do Banco Central do Brasil ao amparo do PPG
\end{abstract}

São Paulo, abril de 2014 


\section{Aprofundando as noções de dependência e envelhecimento em distribuições de probabilidade bivariadas}

Esta versão da tese contém as correções e alterações sugeridas pela Comissão Julgadora durante a defesa da versão original do trabalho, realizada em 21/03/2014. Uma cópia da versão original está disponível no Instituto de Matemática e Estatística da Universidade de São Paulo.

Comissão Julgadora:

- Prof. Dr. Nikolai Kolev (orientador) - IME-USP

- Prof. Dr. Francisco Louzada Neto - ICMC-USP

- Prof. Dr. Antônio Carlos Pedroso de Lima - IME-USP

- Prof. Dr. Cristiano Augusto Coelho Fernandes - PUC-Rio

- Prof. Dr. Narayanaswamy Balakrishnan - McMaster University-Canada 


\section{Deepening the notions of dependence and aging in bivariate probability distributions}

This version of the thesis contains the corrections and adjustments suggested by the Examining Committee during the defense of the original version of the work,

in $03 / 21 / 2014$. A copy of the original version is available at the Institute of Mathematics and Statistics at the University of São Paulo.

Examining Committee:

- Prof. Dr. Nikolai Kolev (advisor) - IME-USP

- Prof. Dr. Francisco Louzada Neto - ICMC-USP

- Prof. Dr. Antônio Carlos Pedroso de Lima - IME-USP

- Prof. Dr. Cristiano Augusto Coelho Fernandes - PUC-Rio

- Prof. Dr. Narayanaswamy Balakrishnan - McMaster University-Canada 


\section{Acknowledgements}

I thank my lovely parents, Aurea and Jayme, who always wanted the best for me. My wife Anna Marina, for her love, patience and ongoing faith on me. My thanks to my advisor Nikolai Kolev, for giving me the opportunity to write this thesis and providing me with excellent research conditions at University of São Paulo, including the opportunity to participate in several scientific conferences. I would like to thank Banco Central do Brasil that, under PPG, provided me the necessary financial support during the last four years. Last, but not least, my thanks to all colleagues of Unibacen, in particular to my friend José Valentim, always available to do his best to help me. 


\section{Resumo}

PINTO, J. A. D. P. Aprofundando as noções de dependência e envelhecimento em distribuições bivariadas de probabilidade. 2014. 174 f. Tese (Doutorado) - Instituto de Matemática e Estatística, Universidade de São Paulo, São Paulo, 2014.

A distribuição bivariada de Marshall-Olkin é estendida, relaxando-se a hipótese de choques exponencialmente distribuídos e assumindo-se dependência entre os choques individuais. Abordagem semelhante é considerada para sua versão dual. Representação por meio de cópula, propriedades probabilísticas e de confiabilidade assim como resultados em valores extremos são então obtidos. A propriedade de falta de memória bivariada é estendida assumindo-se uma função de dependência sem memória. Uma nova classe de distribuições caracterizada por essa propriedade estendida é introduzida. Correspondentes interpretações geométricas, procedimentos de construção, representação estocástica, relação com cópula de sobrevivência e propriedades de confiabilidade são derivadas.

Palavras-chave: Modelo de Marshall-Olkin bivariado, Modelo dual, Representação exponencial, Funçāo de dependência, Envelhecimento bivariado, Cópula, Cópula de sobrevivência, Ordem estocástica, Distribuição bivariada de valores extremos, Medida de Pickands, Função de dependência de Pickands, Taxa de falhas, Gradiente bivariado de riscos, Falta de memória bivariada, Vetor de vidas residuais, Caracterização. 


\section{Abstract}

PINTO, J. A. D. P. Deepening the notions of dependence and aging in bivariate probability distributions. 2014. 174 f. Tese (Doutorado) - Instituto de Matemática e Estatística, Universidade de São Paulo, São Paulo, 2014.

The bivariate Marshall-Olkin distribution is extended, relaxing the assumption of exponentially distributed shocks and assuming dependence between the individual shocks. Similar approach is considered for its dual version. Copula representation, probabilistic and reliability properties as well as extreme value results are obtained. The bivariate lack-of-memory property is extended assuming memoryless dependence function. A new class of distributions characterized by this extended property is introduced. Corresponding geometric interpretations, building procedures, stochastic representation, survival copula relation and reliability properties are derived.

Keywords: Bivariate Marshall-Olkin model, Dual model, Exponential representation, Dependence function, Bivariate aging, Copula, Survival copula, Stochastic order, Bivariate extreme value distribution, Pickands measure, Pickands dependence function, Failure rate, Bivariate hazard gradient, Bivariate lack-of-memory, Residual lifetime vector, Characterization. 


\section{Contents}

List of Abbreviations $\quad$ xi

List of Symbols $\quad$ xiii

List of Figures $\quad$ XV

List of Tables $\quad$ xvii

1 Introduction $\quad 1$

1.1 Objectives . . . . . . . . . . . . . . . . . . . . . 1

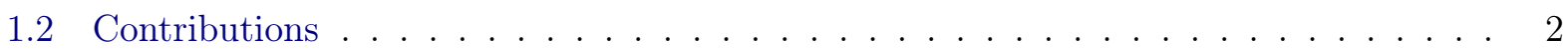

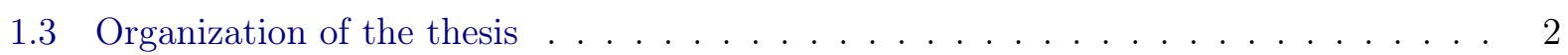

2 Bivariate Marshall-Olkin distribution and lack-of-memory property 5

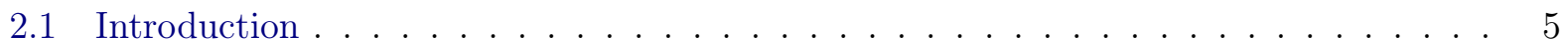

2.2 Gumbel distributions . . . . . . . . . . . . . . . . . . . . . 5

2.3 Bivariate Marshall-Olkin distribution . . . . . . . . . . . . . . . . . 6

2.3.1 Derivations of the Marshall-Olkin distribution . . . . . . . . . . . . . 6

2.3.2 Some probabilistic and statistical properties . . . . . . . . . . . 8

2.3.3 Generalizations of Marshall-Olkin distribution . . . . . . . . . . . . . . . 10

2.4 Alternative concepts of lack-of-memory property . . . . . . . . . . . . . . 10

2.4 .1 Binary operation approach . . . . . . . . . . . . . 10

2.4 .2 Other definitions . . . . . . . . . . . . . . . . . . . . . . 11

3 Extended Marshall-Olkin Model $\quad 15$

3.1 Introduction . . . . . . . . . . . . . . . . . . . . . 15

3.2 Extended Marshall-Olkin model and probabilistic properties . . . . . . . . . . . . . 16

3.2.1 Alternative representation for EMO model . . . . . . . . . . . . . . . . 16

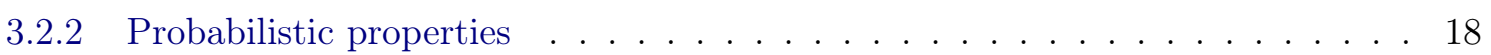

3.3 Survival copula representation and distributional property for residual lifetimes . . . 22

3.3.1 Survival copula of the EMO model . . . . . . . . . . . . . . . . . . 22

3.3.2 Distributional property for residual lifetimes . . . . . . . . . . . . . 26

3.4 Other survival copula representations . . . . . . . . . . . . . . . . . . . . . 27

3.4.1 Alternative survival copula representation . . . . . . . . . . . . . 27

3.4.2 Inverse survival copula representation . . . . . . . . . . . . . . . 28

3.5 An absolutely continuous version of EMO model . . . . . . . . . . . . . . . 31 
3.6 Reliability properties . . . . . . . . . . . . . . . . . . 34

3.6.1 Bivariate lack-of-memory property . . . . . . . . . . . . . . 34

3.6 .2 Weak bivariate increasing failure rate . . . . . . . . . . . . . . 37

3.6 .3 Bivariate increasing hazard rate . . . . . . . . . . . . . . . . . 40

3.7 Extreme value EMO models . . . . . . . . . . . . . . . . . . . . . . . . . 42

3.7.1 Univariate and bivariate extreme value distributions . . . . . . . . . . . 42

3.7.2 Pickands representation for bivariate extreme value copulas . . . . . . . . . . 44

3.7.3 Construction of extreme value EMO models . . . . . . . . . . . . . . . . . . . 44

3.8 Bayesian data analysis with EMO models . . . . . . . . . . . . . . . . . 51

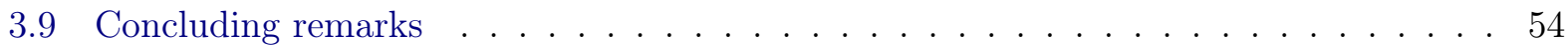

4 Dual extended Marshall-Olkin Model $\quad 57$

4.1 Introduction . . . . . . . . . . . . . . . . . . . . . . 57

4.2 Dual Extended Marshall-Olkin model and probabilistic properties . . . . . . . . . . 58

4.2.1 Model specification and basic probabilistic properties . . . . . . . . . . . . 58

4.2 .2 Copula of the d-EMO distribution . . . . . . . . . . . . . 63

4.2 .3 Preservation of the d-EMO distribution . . . . . . . . . . . . . . . 66

4.3 Stochastic order comparisons . . . . . . . . . . . . . . . 67

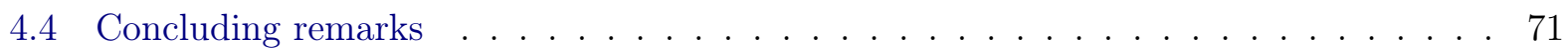

5 Bivariate Distributions with Linear Sum of Hazard Gradient Components $\quad 73$

5.1 Introduction . . . . . . . . . . . . . . . . . . . . . 73

5.2 Model formalization and characterization . . . . . . . . . . . . . . . . 74

5.2 .1 Model specification . . . . . . . . . . . . . . . . . . . 75

5.2 .2 A characterization . . . . . . . . . . . . . . . . . . . . . 79

5.2 .3 Restrictions on the marginal densities . . . . . . . . . . . . . . . . 82

5.2 .4 Geometric interpretations . . . . . . . . . . . . . . . . . 88

5.3 Construction of bivariate distributions belonging to the class $\mathcal{L}(\mathbf{x} ; \mathbf{a}) \ldots \ldots$. . . . . 89

5.3 .1 Bivariate distributions with independent marginals . . . . . . . . . . . . . 89

5.3.2 Bivariate distributions with given marginal failure rates . . . . . . . . . . . 90

5.3 .3 Closure properties of the class $\mathcal{L}(\mathbf{x} ; \mathbf{a}) \ldots \ldots \ldots \ldots \ldots$

5.4 Alternative representations and generalizations of the class $\mathcal{L}(\mathbf{x} ; \mathbf{a}) \ldots \ldots 7$

5.4 .1 Comparable representations . . . . . . . . . . . . . . . . 97

5.4 .2 Extensions . . . . . . . . . . . . . . . . . . . . . . . . . . . 99

5.5 Concluding remarks . . . . . . . . . . . . . . . . . . . 100

6 Linear Sibuya Type Bivariate Lack-of-memory Property 103

6.1 Introduction . . . . . . . . . . . . . . . . . . . . . 103

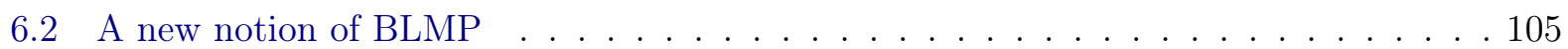

6.2 .1 Linear Sibuya-type BLMP . . . . . . . . . . . . . . . . . 105

6.2.2 Survival copula representations of the class $\mathcal{L}(\mathbf{x} ; \mathbf{a}) \ldots \ldots \ldots$. . . . . . 109

6.3 Complementary patrimony of LS-BLMP . . . . . . . . . . . . . . . 113

6.3.1 Closure properties . . . . . . . . . . . . . . . . . . . 113

6.3 .2 Reliability properties . . . . . . . . . . . . . . . . . . 114 


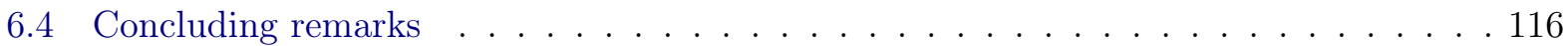

7 Sibuya Type Bivariate Lack-of-memory Property 119

7.1 Introduction . . . . . . . . . . . . . . . . . . . . . . . . 119

7.2 Weaker version of the linear Sibuya-BLMP . . . . . . . . . . . . . . 120

7.2 .1 A characterizing functional equation . . . . . . . . . . . . . . 120

7.2 .2 Related GMO models . . . . . . . . . . . . . . . . . . . . . . . 122

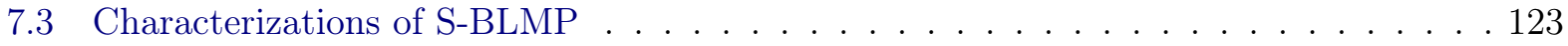

7.3.1 Definition and characterization of the extended class $\mathcal{L}(\mathbf{x} ; \mathbf{A}) \ldots 124$

7.3.2 Equivalence between the class $\mathcal{L}(\mathbf{x} ; \mathbf{A})$ and distributions possessing S-BLMP . 126

7.3.3 Stochastic representation . . . . . . . . . . . . . . . . . . 129

7.3.4 Characterization of absolutely continuous S-BLMP distributions . . . . . . 130

7.4 Generation of distributions possessing S-BLMP . . . . . . . . . . . . . . . . 132

7.4 .1 Closure properties . . . . . . . . . . . . . . . . 132

7.4 .2 Common building scheme . . . . . . . . . . . . . . . . . . . 133

7.5 Bivariate survival functions with non-aging survival copulas . . . . . . . . . 136

7.6 A stronger version of S-BLMP . . . . . . . . . . . . . . . . . . 138

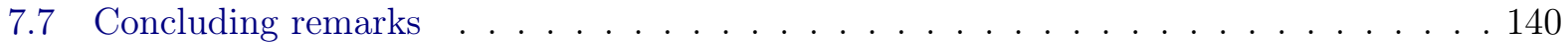

8 Conclusions 143

8.1 Concluding remarks . . . . . . . . . . . . . . . . . . . . . 143

8.2 Future research . . . . . . . . . . . . . . . . . . . . . . . . 144

8.3 Talks, technical reports and submitted papers . . . . . . . . . . . . . 145

$\begin{array}{lr}\text { Bibliography } & 147\end{array}$ 


\title{
List of Abbreviations
}

\author{
MO Marshall-Olkin \\ GMO Generalized Marshall-Olkin \\ EMO Extended Marshall-Olkin \\ d-EMO Dual Extended Marshall-Olkin \\ LMP Lack-of-memory Property \\ BLMP Bivariate Lack-of-memory Property \\ BLMP $_{1} \quad$ Bivariate Lack-of-memory Property Type I \\ $\mathrm{BLMP}_{2} \quad$ Bivariate Lack-of-memory Property Type II \\ LS-BLMP Linear Sibuya-type Bivariate Lack-of-memory Property \\ S-BLMP Sibuya-type Bivariate Lack-of-memory Property \\ NQD Negative Quadrant Dependent \\ PQD Positive Quadrant Dependent \\ IFR Increasing Failure Rate \\ DFR Decreasing Failure Rate \\ BIFR Bivariate Increasing Failure Rate \\ BDFR Bivariate Decreasing Failure Rate \\ BIHR Bivariate Increasing Hazard Rate \\ BDHR Bivariate Decreasing Hazard Rate
}




\title{
List of Symbols
}

\author{
$\min (a, b) \quad$ minimum between $a$ and $b$ \\ $\max (a, b) \quad$ maximum between $a$ and $b$ \\ $\sup \{$.$\} \quad supremum of the elements of the set \{$. \\ $\inf \{$.$\} \quad infimum of the elements of the set \{$. \\ $\mathbb{P}($.$) \quad probability of an event$ \\ $\mathbb{P}(. \mid$.$) \quad conditional probability$ \\ $\mathbb{E}($.$) \quad expectation of an event$ \\ $\mathbb{E}(. \mid$.$) \quad conditional expectation$ \\ $i$ \\ $T_{i}$ \\ $F_{T_{i}}($. \\ natural number that, depending on the context, equals 1,2 or 3 \\ continuous random variable \\ $S_{T_{i}}($.$) \quad survival function of T_{i}$ \\ $f_{T_{i}}($.$) \quad density function of T_{i}$ \\ $r_{T_{i}}($.$) \quad failure rate function of T_{i}$ \\ $H_{T_{i}}($.$) \quad cumulative failure rate function of T_{i}$ \\ $G_{i}($.$) \quad sum of cumulative failure rate functions of T_{i}$ and $T_{3}$ \\ $G_{i}^{-1}($.$) \quad inverse of the sum of cumulative failure rate functions of T_{i}$ and $T_{3}$ \\ $m_{T_{i}}($.$) \quad reversed failure rate function of T_{i}$ \\ $M_{T_{i}}($.$) \quad remaining reversed failure rate function of T_{i}$ \\ $T_{i, t} \quad$ residual lifetime random variable of $T_{i}$ at time $t$ \\ $T_{3(t)} \quad$ inactivity time random variable of $T_{3}$ at time $t$ \\ $F_{T_{1}, T_{2}}(.,$.$) \quad joint distribution function of T_{1}$ and $T_{2}$ \\ $S_{T_{1}, T_{2}}(.,$.$) \quad joint survival function of T_{1}$ and $T_{2}$ \\ $f_{T_{1}, T_{2}}(.,$.$) \quad joint density function of T_{1}$ and $T_{2}$ \\ $H_{T_{1}, T_{2}}(.,$.$) \quad dependence function for S_{T_{1}, T_{2}}(.,$. \\ $G(.,$.$) \quad alternative representation for the dependence function H_{T_{1}, T_{2}}(.,$. \\ $A_{T_{1}, T_{2}}(.,$.$) \quad dependence function for F_{T_{1}, T_{2}}(.,$. \\ $\bar{G}(.,$.$) \quad alternative representation for the dependence function A_{T_{1}, T_{2}}(.,$. \\ $r_{T_{1}, T_{2}}(.,$.$) \quad bivariate failure rate function of T_{1}$ and $T_{2}$ \\ $r_{i}(.,$.$) \quad conditional hazard rate function of T_{i}$ \\ $\mathbf{R}\left(x_{1}, x_{2}\right)(.,$.$) \quad hazard gradient vector$ \\ $r(.,$.$) \quad sum of the components of the hazard gradient vector$ \\ $m_{T_{1}, T_{2}}(.,$.$) \quad bivariate reversed failure rate function of T_{1}$ and $T_{2}$ \\ $m_{i}(.,$.$) \quad conditional reversed hazard rate function of T_{1}$ and $T_{2}$
}


$\bar{G}_{i}($.

$\bar{G}_{i}^{-1}($.

$\mathbf{T}_{12(t)}$

$X_{i}$

$F_{X_{i}}($.

$S_{X_{i}}($.

$f_{X_{i}}($.

$F_{X_{i}}^{-1}($.

$S_{X_{i}}^{-1}($.

$H_{X_{i}}($.

$\mathrm{X}$

$F_{X_{1}, X_{2}}(.,$.

$C_{X_{1}, X_{2}}(.,$.

$S_{X_{1}, X_{2}}(.,$.

$S_{X_{1}, X_{2}}^{a c}(.,$.

$S_{X_{1}, X_{2}}^{s i}(.,$.

$\bar{C}_{X_{1}, X_{2}}(.,$.

$\mathbf{X}_{\mathbf{t}}$

$\mathbf{X}_{(t)}$

$\mathbf{M}\left(x_{1}, x_{2}\right)(.,$.

$M_{i}(.,$.

$M(.,$.

$T_{i}(.,$.

$\operatorname{Cov}(.,$.

$\Omega$ sum of remaining reversed failure rate functions of $T_{i}$ and $T_{3}$

inverse of the sum of remaining reversed failure rate functions of $T_{i}$ and $T_{3}$ inactivity times vector of $\left(T_{1}, T_{2}\right)$ at time $t$

continuous random variable

distribution function of $X_{i}$

survival function of $X_{i}$

density function of $X_{i}$

inverse of the distribution function of $X_{i}$

inverse of the survival function of $X_{i}$

cumulative failure rate function of $X_{i}$

bivariate random vector $\left(X_{1}, X_{2}\right)$

joint distribution function of $\left(X_{1}, X_{2}\right)$

copula of $\left(X_{1}, X_{2}\right)$

joint survival function of $\left(X_{1}, X_{2}\right)$

absolutely continuous component of the joint survival function of $\left(X_{1}, X_{2}\right)$

singular component of the joint survival function of $\left(X_{1}, X_{2}\right)$

survival copula of $\left(X_{1}, X_{2}\right)$

bivariate random vector of residual lifetimes of $\left(X_{1}, X_{2}\right)$ at time $t$

bivariate random vector of inactivity times of $\left(X_{1}, X_{2}\right)$ at time $t$

mean residual lifetime vector

$\mathrm{i}^{\text {th }}$ component of the mean residual lifetime vector

product moment residual life function

$\mathrm{i}^{\text {th }}$ component of the vector valued reciprocal of the coordinate subtangent covariance between two random variables

set $\left\{\left(x_{1}, x_{2}\right) \in \mathbb{R}_{+}^{2} \mid x_{1}=x_{2}=x\right\}$ 


\section{List of Figures}

2.1 "Fatal shock" model in Marshall-Olkin distribution. . . . . . . . . . . . . . . . . 6

$3.1 \mathrm{MO}$, GMO and EMO models compared. . . . . . . . . . . . . . 17

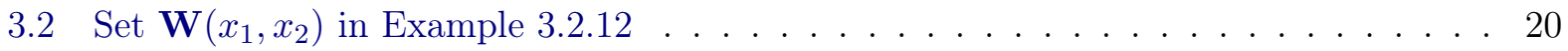

3.3 Dependence analysis of the EMO model in Example 3.3.5 . . . . . . . . . . . . 25

3.4 UEFA Champion's League data - Meintanis (2007) . . . . . . . . . . . . . . 52

4.1 "Fatal shock" model in dual generalized Marshall-Olkin distribution. . . . . . . . . . 57

4.2 Dual GMO and dual EMO models compared. . . . . . . . . . . . . . . . . . 59

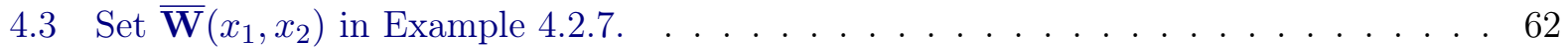

4.4 Dependence analysis of the d-EMO distribution in Example $4.2 .16 \ldots 6$

5.1 Paths of integration $\mathcal{C}_{1}$ and $\mathcal{C}_{2}$ when $x_{1} \geq x_{2} \geq 0 \ldots \ldots \ldots$

5.2 Geometric representation of the class $\mathcal{L}(\mathbf{x} ; \mathbf{a}) \ldots \ldots \ldots$

6.1 An interpretation for relation $(6.3) \ldots \ldots \ldots \ldots \ldots$

7.1 Equivalence relations for S-BLMP and class $\mathcal{L}(\mathbf{x} ; \mathbf{A}) \ldots \ldots \ldots \ldots$

7.2 The three BLMP concepts and corresponding examples of distributions . . . . . . . 141 


\section{List of Tables}

2.1 Examples of univariate survival functions under different binary operations . . . . . 11

2.2 Examples of bivariate survival functions under different binary operations . . . . . . 11

3.1 Absolutely continuous survival functions for lifetimes $\left(X_{1}, X_{2}\right) \ldots \ldots \ldots$

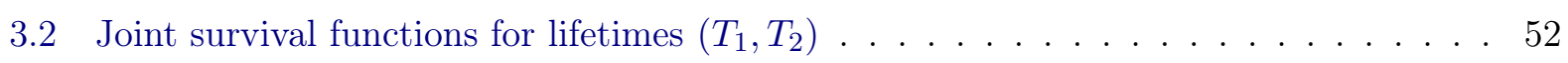

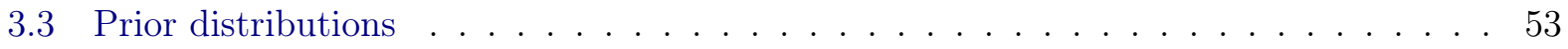

3.4 Model (M1) - Gumbel (1960) type I bivariate exponential . . . . . . . . . . . . . 53

3.5 Model (M2) - Block and Basu (1974) bivariate exponential . . . . . . . . . . . . . 53

3.6 Model (M3) - Marshall and Olkin (1967) bivariate exponential . . . . . . . . . . 53

3.7 Model (M4): EMO-1 (Gumbel (1960) type I for $\left.\left(T_{1}, T_{2}\right)\right) \ldots \ldots \ldots$

3.8 Model (M5) - EMO-2 (Block and Basu (1974) for $\left.\left(T_{1}, T_{2}\right)\right) \ldots \ldots \ldots \ldots$

3.9 Monte Carlo estimates for DIC . . . . . . . . . . . . . . . . . . 54

5.1 Basic expressions for independence case . . . . . . . . . . . . . . . . . 90

8.1 Conferences, workshops and talks . . . . . . . . . . . . . . . 145

8.2 Technical Reports at Institute of Mathematics and Statistics (USP) . . . . . . . 146 


\section{Chapter 1}

\section{Introduction}

A nonnegative continuous random variable $X$ follows the exponential distribution with parameter $\lambda>0$ if its density function is given by

$$
f_{X}(x)=\lambda \exp \{-\lambda x\}
$$

The exponential distribution plays a central role in Statistics, not only for its properties, but also for its applications and a thorough exposition on this probability distribution can be found in Chapter 1 of Galambos and Kotz (1978) and in Balakrishnan and Basu (1995).

Among the several properties of the exponential distribution, one particularly important for this thesis refers to the lack-of-memory. Denoting $S_{X}(x)=\mathbb{P}(X>x)$ the survival function of $X$, the lack-of-memory property is given by the functional equation

$$
S_{X}(x+y)=S_{X}(x) S_{X}(y)
$$

for all $x, y \geq 0$. In fact, if $X$ is a nonnegative continuous random variable, then $X$ follows an exponential distribution if and only if its survival function satisfies (1.2). In other words, among the continuous univariate distributions, the lack-of-memory property characterizes the exponential distribution.

The lack-of-memory property is fundamental for obtaining a particular bivariate exponential distribution, namely, the bivariate Marshall-Olkin distribution, see Marshall and Olkin (1967).

In this thesis, two different extensions will be obtained, both having the bivariate Marshall-Olkin model as the starting point. The first extension is directly concerned with the distribution and its dual version. The second one has to do with the generalization of the bivariate lack-of-memory property introduced in Marshall and Olkin (1967).

\section{$1.1 \quad$ Objectives}

The objective of this thesis is twofold. In the first part, consisting of Chapters 3 and 4 , we extend the Marshall-Olkin distribution and its dual version, by allowing dependence between the subjacent individual shocks, and study the properties of the resulting models. In the second part, covered on Chapters 5 to 7, we extend the bivariate lack-of-memory property introduced in Marshall and Olkin (1967), by requiring only a memoryless dependence function, and also study the properties of the 
class of distributions that satisfy this weaker property.

\subsection{Contributions}

The main contributions of this thesis are:

- In Chapter 3 we extend the Marshall-Olkin distribution, introduced in Marshall and Olkin (1967), relaxing the assumptions of exponentially distributed and independent individual shocks. For the proposed model we obtain the corresponding survival copula expression, an inverse copula representation, probabilistic properties, an absolutely continuous version and related aging properties, as well an extreme value result;

- In Chapter 4 we extend the dual generalized Marshall-Olkin distribution of Fang and Li (2013) relaxing the assumption of independence between individual shocks. For the obtained model we obtain the corresponding copula expression, probabilistic and stochastic order properties;

- In Chapter 5 we introduce a new class $\mathcal{L}(\mathbf{x} ; \mathbf{a})$ of nonnegative continuous distributions characterized by a linear form for the sum of the components of the hazard gradient vector. This new class contains all distributions which possess the bivariate lack-of-memory property, the Gumbel's type I bivariate exponential distribution and many others. These distributions exhibit a characteristic aging behaviour. We characterize the members of this new class and provide a methodology for obtaining them;

- In Chapter 6 we introduce a new type of bivariate lack-of-memory property, the linear Sibuya bivariate lack-of-memory property (LS-BLMP), weaker than the classical property introduced in Marshall and Olkin (1967). We require a memory-less dependence function and additionally impose a natural aging condition on marginals. This property characterizes the class $\mathcal{L}(\mathbf{x} ; \mathbf{a})$ introduced in Chapter 5 . Closure and reliability properties are obtained;

- In Chapter 7 we introduce the Sibuya bivariate lack-of-memory property (S-BLMP), a weaker form for LS-BLMP. We also introduce the class $\mathcal{L}(\mathbf{x} ; \mathbf{A})$, composed by nonnegative continuous distributions characterized by a separable form for the sum of the components of the hazard gradient vector, generalizing the class $\mathcal{L}(\mathbf{x} ; \mathbf{a})$. We show the equivalence between S-BLMP and $\mathcal{L}(\mathbf{x} ; \mathbf{A})$. Concerning the members of $\mathcal{L}(\mathbf{x} ; \mathbf{A})$, we provide a stochastic representation, characterize the absolutely continuous ones as well as propose a general building scheme. We also generalize the S-BLMP in a way that parallels the approach of Oakes (2005).

\subsection{Organization of the thesis}

In Chapter 2 we review the bivariate Marshall-Olkin distribution and the various definitions of lack-of-memory property. In Chapter 3 we extend the Marshall-Olkin distribution of Marshall and Olkin (1967) and in Chapter 4, the dual generalized Marshall-Olkin distribution of Fang and Li (2013). In Chapter 5 we introduce a new class of distributions, denoted by $\mathcal{L}(\mathbf{x} ; \mathbf{a})$ and in Chapter 6 we consider a new type of bivariate lack-of-memory property, the linear Sibuya bivariate lack-of-memory property (LS-BLMP). In Chapter 7 the Sibuya bivariate lack-of-memory property (S-BLMP) and 
a wider class of distributions $\mathcal{L}(\mathbf{x} ; \mathbf{A})$ are defined and studied. We also generalize the S-BLMP. Conclusions are presented in Chapter 8. 


\section{Chapter 2}

\section{Bivariate Marshall-Olkin distribution and lack-of-memory property}

\section{$2.1 \quad$ Introduction}

According to Galambos and Kotz (1978), multivariate versions of the exponential distribution took time to be introduced due to the late development of statistical models based on the univariate exponential distribution as well as the strong influence of the multivariate normal distribution on theoretical and applied Statistics. The earliest bivariate extensions of the exponential distribution were motivated by extreme value theory.

Differently from the normal distribution, there is not a unique bivariate exponential distribution. Bivariate exponential distributions should have marginals exponentially distributed, but this is not the rule and the distributions of Freund (1961), Friday and Patil (1977) and Arnold and Strauss (1988) are sometimes denominated bivariate extensions of the exponential distribution.

In this chapter we will consider the bivariate exponential distributions proposed by Gumbel (1960) and Marshall and Olkin (1967). For other bivariate (extensions of) exponential distributions, we refer the reader to Basu (1988), Kotz et al. (2000) and Balakrishnan and Lai (2009).

\subsection{Gumbel distributions}

Gumbel (1960) suggested three different bivariate exponential distributions, also known as Type I, II and III, with support on $\left\{\left(x_{1}, x_{2}\right) \mid x_{1}, x_{2} \geq 0\right\}$, and exponential marginals with unitary parameter. Denoting bivariate survival functions as $S_{X_{1}, X_{2}}\left(x_{1}, x_{2}\right)=\mathbb{P}\left(X_{1}>x_{1}, X_{2}>x_{2}\right)$, the distributions proposed in Gumbel (1960) are respectively given by

$$
\begin{gathered}
S_{X_{1}, X_{2}}\left(x_{1}, x_{2}\right)=\exp \left\{-x_{1}-x_{2}-\theta x_{1} x_{2}\right\}, \theta \in[0,1], \\
S_{X_{1}, X_{2}}\left(x_{1}, x_{2}\right)=\exp \left\{-x_{1}\right\}+\exp \left\{-x_{2}\right\} \\
+\left(1-\exp \left\{-x_{1}\right\}\right)\left(1-\exp \left\{-x_{2}\right\}\right)\left[1+\alpha \exp \left\{-x_{1}-x_{2}\right\}\right]-1, \alpha \in[-1,1], \\
S_{X_{1}, X_{2}}\left(x_{1}, x_{2}\right)=\exp \left\{-\left(x_{1}^{m}+x_{2}^{m}\right)^{\frac{1}{m}}\right\}, m \geq 1 .
\end{gathered}
$$


The Gumbel's Type I bivariate exponential distribution (2.1) has been differently characterized in the literature, for example, Johnson and Kotz (1975), Raja Rao et al. (1993), Nair and Nair (1988b), Nair and Nair (1988a), Roy (2002a) and Roy (2004). All these characterizations share a common aspect, a new definition for bivariate lack-of-memory property, see Section 2.4.

By its turn, the Gumbel's Type III bivariate exponential distribution (2.3) has applications in extreme value theory, see Example 3.7.18.

These bivariate distributions have more general versions, where the marginals are exponentially distributed, but the parameter is no longer unitary, see relations (3.12) and (5.3), for example, with corresponding survival copulas given by (3.42) and (3.17). In Balakrishnan and Lai (2009) and Kotz et al. (2000) one can find an extensive analysis of these distributions.

\subsection{Bivariate Marshall-Olkin distribution}

Marshall and Olkin (1967) introduced a bivariate exponential distribution with survival function given by

$$
S_{X_{1}, X_{2}}\left(x_{1}, x_{2}\right)=\exp \left\{-\lambda_{1} x_{1}-\lambda_{2} x_{2}-\lambda_{3} \max \left(x_{1}, x_{2}\right)\right\},
$$

for all $x_{1}, x_{2} \geq 0$ and $\lambda_{i}>0, i=1,2,3$. Four different approaches that can be used to obtain this distribution are shown in the sequel.

\subsubsection{Derivations of the Marshall-Olkin distribution}

\section{"Fatal shock" model}

Consider a two component system subject to three different shocks that, upon occurrence, destroy the hit component. The first shock only hits the first component, the same for the second shock, while the third one hits both components simultaneously, see Figure 2.1. For this reason, the first two shocks are known as individual shocks and the third one is denominated common shock.

The shocks are governed by independent Poisson processes, $Z_{1}\left(t, \lambda_{1}\right), Z_{2}\left(t, \lambda_{2}\right)$ and $Z_{3}\left(t, \lambda_{3}\right)$, where $Z_{i}, i=1,2$, denote the individual shocks, $Z_{3}$ the common shock and $\lambda_{i}, i=1,2,3$, the parameters of the corresponding Poisson processes.

Denoting by $X_{1}$ and $X_{2}$ the lifetimes of the two components, their joint survival function is given by the Marshall-Olkin distribution (2.4).

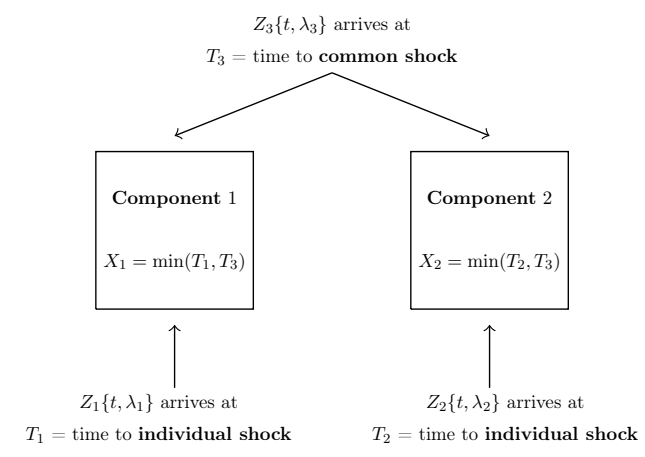

Figure 2.1: "Fatal shock" model in Marshall-Olkin distribution. 


\section{Non-fatal shock models}

In this second approach a two component system is still considered, but the shocks may (or may not) destroy the hit component, changing (or not) its state from 1 (working) for 0 (not working).

The state of the system is described by ordered pairs $(1,1),(0,1),(1,0)$ and $(0,0)$, where the first position refers to the state of the first component and the second position to the state of the second one. The shock $i, i=1,2$, can destroy component $i$ with probability $p_{i}$. The third shock can change the state of the system from $(1,1)$ to $(0,0),(0,1),(1,0)$ or $(1,1)$ with probabilities $p_{00}, p_{01}$, $p_{10}$ and $p_{11}$, respectively.

From this formulation it is possible to recover the Marshall-Olkin distribution (2.4), but not so directly as in the previous formulation. The "Fatal shock" model is a particular case, when $p_{1}=p_{2}=1$ and $p_{00}=1$.

\section{Residual lifetime independent of age}

In this third formulation, Marshall and Olkin (1967) initially tried to extend the univariate lack-of-memory property to the bivariate dimension considering the functional equation

$$
S_{X_{1}, X_{2}}\left(x_{1}+t_{1}, x_{2}+t_{2}\right)=S_{X_{1}, X_{2}}\left(x_{1}, x_{2}\right) S_{X_{1}, X_{2}}\left(t_{1}, t_{2}\right)
$$

for all $x_{1}, x_{2} \geq 0$ and $t_{1}, t_{2} \geq 0$.

Meanwhile, condition (2.5) is too strong and the only continuous solution is

$$
S_{X_{1}, X_{2}}\left(x_{1}, x_{2}\right)=\exp \left\{-b_{1} x_{1}-b_{2} x_{2}\right\}, b_{1}, b_{2}>0
$$

i.e., $X_{1}$ and $X_{2}$ are independent and exponentially distributed.

A weaker version, obtained letting $t_{1}=t_{2}=t$ in (2.5) was considered, resulting in the functional equation

$$
S_{X_{1}, X_{2}}\left(x_{1}+t, x_{2}+t\right)=S_{X_{1}, X_{2}}\left(x_{1}, x_{2}\right) S_{X_{1}, X_{2}}(t, t),
$$

for all $x_{1}, x_{2} \geq 0$ and $t \geq 0$. Relation (2.6) admits the probabilistic interpretation

$$
\mathbb{P}\left(X_{1}>x_{1}+t, X_{2}>x_{2}+t \mid x_{1}>t, x_{2}>t\right)=\mathbb{P}\left(X_{1}>x_{1}, X_{2}>x_{2}\right) .
$$

Remark 2.3.1. Relation (2.6), or its equivalent version (2.7), characterizes the earliest version of the bivariate lack-of-memory property. In fact, bivariate survival functions that satisfy (2.6), or (2.7), have the bivariate lack-of-memory property.

The continuous solution of the functional equation (2.6) is

$$
S_{X_{1}, X_{2}}\left(x_{1}, x_{2}\right)= \begin{cases}\exp \left\{-\theta x_{2}\right\} S_{X_{1}}\left(x_{1}-x_{2}\right), & \text { if } x_{1} \geq x_{2} \geq 0 \\ \exp \left\{-\theta x_{1}\right\} S_{X_{2}}\left(x_{2}-x_{1}\right), & \text { if } x_{2} \geq x_{1} \geq 0\end{cases}
$$

where $\theta>0$.

Imposing marginals exponentially distributed in (2.8) the Marshall-Olkin distribution (2.4) is obtained. One can consider other marginal distributions in relation (2.8), such as mixtures of exponential distributions. Meanwhile, restrictions on the marginals have to be considered so that (2.8) 
is a proper survival function, see Ghurye and Marshall (1984) and Kulkarni (2006).

Remark 2.3.2. Block (1977) characterized the lack-of-memory property based on a previous result in Block and Basu (1974): let $\left(X_{1}, X_{2}\right)$ be a bivariate nonnegative random vector with absolutely continuous marginal distributions and consider the random variables $X=\min \left(X_{1}, X_{2}\right)$ and $D=$ $X_{1}-X_{2}$. The lack-of-memory property holds true if and only if $X$ and $D$ are independent and $X$ is exponentially distributed.

\section{Stochastic representation}

Marshall and Olkin (1967) showed that the nonnegative continuous bivariate random vector $\left(X_{1}, X_{2}\right)$ follows the Marshall-Olkin distribution if and only if there exist random variables $T_{1}, T_{2}$ and $T_{3}$ independent and exponentially distributed such that

$$
\left(X_{1}, X_{2}\right)=\left(\min \left(T_{1}, T_{3}\right), \min \left(T_{2}, T_{3}\right)\right),
$$

providing a fourth characterization of this distribution. In Section 4 of Marshall and Olkin (1967) these different characterizations are used to obtain the multivariate version of the distribution.

Remark 2.3.3. Other characterizations of the Marshall-Olkin distribution (2.4) can be found in the literature, for example Obretenov (1985) and Roy and Mukherjee (1989).

\subsubsection{Some probabilistic and statistical properties}

\section{Distributional properties}

The Marshall-Olkin distribution can be decomposed into an absolutely continuous component, $S_{X_{1}, X_{2}}^{a c}\left(x_{1}, x_{2}\right)$, and a singular one, $S_{X_{1}, X_{2}}^{s i}\left(x_{1}, x_{2}\right)$, with support on the set

$$
\Omega=\left\{\left(x_{1}, x_{2}\right) \in \mathbb{R}_{+}^{2} \mid x_{1}=x_{2}=x\right\}
$$

Denoting $\alpha=\mathbb{P}\left(X_{1}=X_{2}\right)$, for the Marshall-Olkin distribution (2.4) we have

$$
\alpha=\mathbb{P}\left(T_{3}<\min \left(T_{1}, T_{2}\right)\right)=\frac{\lambda_{3}}{\lambda_{1}+\lambda_{2}+\lambda_{3}}>0 .
$$

The distribution admits the Lebesgue decomposition

$$
S_{X_{1}, X_{2}}\left(x_{1}, x_{2}\right)=(1-\alpha) S_{X_{1}, X_{2}}^{a c}\left(x_{1}, x_{2}\right)+\alpha S_{X_{1}, X_{2}}^{s i}\left(\max \left(x_{1}, x_{2}\right)\right),
$$

where

$$
S_{X_{1}, X_{2}}^{s i}\left(\max \left(x_{1}, x_{2}\right)\right)=\exp \left\{-\left(\lambda_{1}+\lambda_{2}+\lambda_{3}\right) \max \left(x_{1}, x_{2}\right)\right\} .
$$

The distribution of $X=\min \left(X_{1}, X_{2}\right)$ is exponential with parameter $\lambda=\lambda_{1}+\lambda_{2}+\lambda_{3}$. The correlation coefficient $\rho$ is such that $\rho=\alpha=\mathbb{P}\left(X_{1}=X_{2}\right)$ and is always nonnegative, i.e., $0 \leq \rho \leq 1$.

Moreover, we have

$$
\begin{aligned}
0 \leq S_{X_{1}, X_{2}}\left(x_{1}, x_{2}\right)-S_{X_{1}}\left(x_{1}\right) S_{X_{2}}\left(x_{2}\right) & =\exp \left\{-\lambda_{1} x_{1}-\lambda_{2} x_{2}-\lambda_{3} \max \left(x_{1}, x_{2}\right)\right\} \\
& \times\left(1-\exp \left\{-\lambda_{3} \min \left(x_{1}, x_{2}\right)\right\}\right),
\end{aligned}
$$


with implications on quadrant dependence properties, see Section 3.2.2.

\section{Survival copula representation}

Definition 2.3.4 (Nelsen (2006)). Copula is a function $C:[0,1]^{2} \rightarrow[0,1]$ with the following properties:

1. for all $u$ and $v$ in $[0,1], C(u, 0)=0=C(0, v), C(u, 1)=u$ and $C(1, v)=v$;

2. for all $u_{1}, u_{2}, v_{1}$ e $v_{2}$ in $[0,1]$ such that $u_{1} \leq u_{2}$ and $v_{1} \leq v_{2}$, we have $C\left(u_{2}, v_{2}\right)-C\left(u_{2}, v_{1}\right)-$ $C\left(u_{1}, v_{2}\right)+C\left(u_{1}, v_{1}\right) \geq 0$.

The survival copula $\bar{C}(u, v)$, corresponding to the copula $C(u, v)$, can be obtained from the relation

$$
\bar{C}(u, v)=u+v-1+C(1-u, 1-v) .
$$

Nelsen (2006) provides the expression of the Marshall-Olkin survival copula, also known as Generalized Cuadras-Augé, as

$$
\bar{C}_{\eta_{1}, \eta_{2}}(u, v)=\min \left(u^{1-\eta_{1}} v, u v^{1-\eta_{2}}\right)= \begin{cases}u^{1-\eta_{1}} v, & \text { if } \quad u^{\eta_{1}} \geq v_{2}^{\eta}, \\ u v^{1-\eta_{2}}, & \text { if } \quad u^{\eta_{1}} \leq v^{\eta_{2}}\end{cases}
$$

where $0<\eta_{1}, \eta_{2}<1,(u, v) \in[0,1]^{2}$. This survival copula can be obtained from (2.4) considering that $\bar{C}_{\eta_{1}, \eta_{2}}(u, v)=S_{X_{1}, X_{2}}\left(S_{X_{1}}^{-1}(u), S_{X_{2}}^{-1}(v)\right), S_{X_{1}}\left(x_{1}\right)=\exp \left\{-\left(\lambda_{1}+\lambda_{3}\right) x_{1}\right\}, S_{X_{2}}\left(x_{2}\right)=\exp \left\{-\left(\lambda_{2}+\right.\right.$ $\left.\left.\lambda_{3}\right) x_{2}\right\}, \eta_{1}=\frac{\lambda_{3}}{\lambda_{1}+\lambda_{3}}$ and $\eta_{2}=\frac{\lambda_{3}}{\lambda_{2}+\lambda_{3}}$.

\section{Parameter estimation}

Given an independent and identically distributed sample $\left(x_{1 j}, x_{2 j}\right), j=1, \ldots n$, of a MarshallOlkin distribution (2.4), consistent estimators for $\lambda_{i}, i=1,2,3$, were proposed by Arnold (1968).

Bemis et al. (1972) obtained the maximum likelihood estimators. Denoting $N_{k}, k=1,2$ the number of observations such that $x_{k j}>x_{(3-k) j}$, the estimators are unique whenever $N_{1}+N_{2}<n$ and $N_{1} N_{2}>0$. Bemis et al. (1972) also proposed a test for the symmetric Marshall-Olkin distribution, i.e., a test for $\lambda_{1}=\lambda_{2}$. Further, the authors obtained moment based estimators.

Proschan and Sullo (1976) introduced estimators based on the first iteration of the maximum of the log-likelihood function. Karlis (2003) obtained the maximum likelihood estimators via EM algorithm. In a related topic, Meintanis (2007) proposed a test of fit for Marshall-Olkin distributions.

Under the assumption of stochastic independence among the parameters, Bayesian inference procedures for non-identical marginals were developed in Shamseldin (1979) and for identical marginals in Shamseldin and Press (1984). Peña and Gupta (1990) obtained Bayesian estimators allowing for dependence among the parameters.

Balakrishnan and Lai (2009) and Kotz et al. (2000) discuss other aspects related with parameter estimation of Marshall-Olkin distribution as well as indicate further literature on this topic. 


\subsubsection{Generalizations of Marshall-Olkin distribution}

The assumption of independent and exponentially distributed shocks brings mathematical tractability and nice properties to the Marshall-Olkin distribution. However, in many practical situations, such assumptions may not hold true.

In Chapter 3 the Generalized Marshall-Olkin model of Li and Pellerey (2011) will be addressed. Such model, relaxing the assumption of exponentially distributed shocks, not only enlarges the field of applications, but also shares some properties with the Marshall-Olkin distribution.

In the same chapter, a new model will be introduced, the extended Marshall-Olkin model. In this model we relax the assumption of independence between the individual shocks in Li and Pellerey (2011). Following a similar approach, in Chapter 4 we will introduce the dual extended MarshallOlkin model, extending the dual generalized Marshall-Olkin distribution of Fang and Li (2013).

\subsection{Alternative concepts of lack-of-memory property}

The bivariate lack-of-memory property was introduced by Marshall and Olkin (1967) and all bivariate distributions with such a property have to satisfy the functional equation (2.6), or its equivalent version (2.7). As shown in this section, these equations became the starting point for generalizations and alternative formulations for bivariate lack-of-memory property.

\subsubsection{Binary operation approach}

Considering an associative binary operation $\oplus$, i.e., $(x \oplus y) \oplus z=x \oplus(y \oplus z)$, Muliere and Scarsini (1987) generalized the functional equation (2.7) as

$$
\mathbb{P}\left(X_{1}>x_{1} \oplus t, X_{2}>x_{2} \oplus t, \mid X_{1}>t, X_{2}>t\right)=\mathbb{P}\left(X_{1}>x_{1}, X_{2}>x_{2}\right)
$$

for all $x_{1}, x_{2} \geq 0$ and $t \geq 0$. The same binary operation $\oplus$ was additionally assumed to the marginal distributions

$$
\begin{aligned}
& \mathbb{P}\left(X_{1}>x_{1} \oplus t \mid X_{1}>t\right)=\mathbb{P}\left(X_{1}>x_{1}\right), \\
& \mathbb{P}\left(X_{2}>x_{2} \oplus t \mid X_{2}>t\right)=\mathbb{P}\left(X_{2}>x_{2}\right),
\end{aligned}
$$

for all $x_{1}, x_{2} \geq 0$ and $t \geq 0$.

If the binary operation $\oplus$ is reducible, i.e., $x \oplus y=x \oplus z$ or $y \oplus w=z \oplus w$ if and only if $z=y$, then the continuous solution of $(x \oplus y) \oplus z=x \oplus(y \oplus z)$ is given in Aczèl (1966): $x \oplus y=g^{-1}(g(x)+g(y))$, provided $x, y$ and $x \oplus y$ belong to the same interval and the function $g(x)$ is continuous and strictly monotone. The solution $g(x)$ is determined up to a multiplicative constant so that $g(x)$ can be considered strictly increasing. It is also assumed the existence of an identity element $e$ such that $x \oplus e=x$.

The marginal survival functions that solve (2.17) are given by

$$
S_{X_{i}}\left(x_{i}\right)=\exp \left\{-\lambda_{i} g\left(x_{i}\right)\right\}, \lambda_{i}>0, g^{-1}(0)<x_{i}<g^{-1}(\infty), i=1,2 .
$$

Different binary operations $\oplus$ generate different univariate distributions, as shown in Table 2.1. 
Table 2.1: Examples of univariate survival functions under different binary operations

\begin{tabular}{|c|c|c|}
\hline$x \oplus y$ & $S_{X_{i}}\left(x_{i}\right)$ & Survival function \\
\hline$x+y$ & $\exp \{-\lambda s\}$ & Exponential \\
$x * y$ & $s^{-\lambda}$ & Pareto \\
$\sqrt[\alpha]{\left(x^{\alpha}+y^{\alpha}\right)}, \alpha>0$ & $\exp \left\{-\lambda s^{\alpha}\right\}$ & Weibull \\
\hline
\end{tabular}

The corresponding joint survival functions, solutions of (2.16), are

$$
S_{X_{1}, X_{2}}\left(x_{1}, x_{2}\right)=\exp \left\{-\lambda_{1} g\left(x_{1}\right)-\lambda_{2} g\left(x_{2}\right)-\lambda_{3} g\left(\max \left(x_{1}, x_{2}\right)\right)\right\}, \lambda_{i}>0, i=1,2,3
$$

Similarly, different binary operation $\oplus$ generate different bivariate distributions, as shown in Table 2.2.

Table 2.2: Examples of bivariate survival functions under different binary operations

\begin{tabular}{|c|c|c|}
\hline$x \oplus y$ & $S_{X_{1}, X_{2}}\left(x_{1}, x_{2}\right)$ & Survival function \\
\hline$x+y$ & $\exp \left\{-\lambda_{1} x_{1}-\lambda_{2} x_{2}-\lambda_{3} \max \left(x_{1}, x_{2}\right)\right\}$ & Marshall-Olkin \\
$x * y$ & $x_{1}^{-\lambda_{1}} x_{2}^{-\lambda_{2}}\left[\max \left(x_{1}, x_{2}\right)\right]^{-\lambda_{3}}$ & Bivariate Pareto \\
$\sqrt[\alpha]{\left(x^{\alpha}+y^{\alpha}\right)}, \alpha>0$ & $\exp \left\{-\lambda_{1} x_{1}^{\alpha}-\lambda_{2} x_{2}^{\alpha}-\lambda_{3} \max \left(x_{1}^{\alpha}, x_{2}^{\alpha}\right)\right\}$ & Bivariate Weibull \\
\hline
\end{tabular}

These bivariate survival functions share several properties with Marshall-Olkin distribution: a singular component with support on $\Omega=\left\{\left(x_{1}, x_{2}\right) \in \mathbb{R}_{+}^{2} \mid x_{1}=x_{2}=x\right\} ; X_{1}, X_{2}$ and $X=$ $\min \left(X_{1}, X_{2}\right)$ share the same distribution, but with different parameters; the stochastic representation $(2.9)$ still holds true.

Muliere and Scarsini (1987) also considered different binary operations $\oplus_{1}$ and $\oplus_{2}$ in (2.7),

$$
\mathbb{P}\left(X_{1}>x_{1} \oplus_{1} t, X_{2}>x_{2} \oplus_{2} t \mid X_{1}>t, X_{2}>t\right)=\mathbb{P}\left(X_{1}>x_{1}, X_{2}>x_{2}\right)
$$

and imposed the same binary operations to the marginals,

$$
\begin{aligned}
& \mathbb{P}\left(X_{1}>x_{1} \oplus_{1} t \mid X_{1}>t\right)=\mathbb{P}\left(X_{1}>x_{1}\right), \\
& \mathbb{P}\left(X_{2}>x_{2} \oplus_{2} t \mid X_{2}>t\right)=\mathbb{P}\left(X_{2}>x_{2}\right),
\end{aligned}
$$

for all $x_{1}, x_{2} \geq 0$ and $t \geq 0$. In this more general formulation, all the bivariate survival functions obtained share the Marshall-Olkin survival copula (2.15).

\subsubsection{Other definitions}

Other definitions for the bivariate lack-of-memory property have been formulated. Raja Rao et al. (1993) introduced the setting the clock back to zero property. To emphasize the role of the vector parameter on the joint distribution, they write the joint survival function as $S_{X_{1}, X_{2}}\left(x_{1}, x_{2}, \theta\right)=$ $\mathbb{P}\left(X_{1}>x_{1}, X_{2}>x_{2} \mid \theta\right)$, where $\theta$ is the vector parameter. When $\theta$ belongs to a parameter space $\boldsymbol{\Theta}$, this defines a class of bivariate survival functions $\left\{S_{X_{1}, X_{2}}\left(x_{1}, x_{2}, \theta\right), x \geq 0, y \geq 0, \theta \in \boldsymbol{\Theta}\right\}$.

According to Raja Rao et al. (1993), a class of distributions has the setting the clock back to zero property if for each $\theta \in \mathbf{\Theta}, x_{0}>0$ and for all $x_{1}, x_{2} \geq 0$, the survival function satisfies the pair 
of equations

$$
\frac{S_{X_{1}, X_{2}}\left(x_{1}+x_{0}, x_{0}, \theta\right)}{S_{X_{1}, X_{2}}\left(x_{0}, x_{0}, \theta\right)}=S_{X_{1}, X_{2}}\left(x_{1}, x_{0}, \theta^{*}\right), \quad \text { and } \quad \frac{S_{X_{1}, X_{2}}\left(x_{0}, x_{2}+x_{0}, \theta\right)}{S_{X_{1}, X_{2}}\left(x_{0}, x_{0}, \theta\right)}=S_{X_{1}, X_{2}}\left(x_{0}, x_{2}, \theta^{* *}\right),
$$

where $\theta^{*}=\theta^{*}\left(x_{0}\right) \in \boldsymbol{\Theta}$ and $\theta^{* *}=\theta^{* *}\left(x_{0}\right) \in \boldsymbol{\Theta}$.

Some examples of bivariate distributions with this property are the Marshall-Olkin distribution (2.4), the Gumbel's Type I bivariate exponential distribution (2.1) and the bivariate Pareto distribution with survival function $S_{X_{1}, X_{2}}\left(x_{1}, x_{2}, \theta\right)=\left(1+\sigma_{1} x_{1}+\sigma_{2} x_{2}\right)^{-\alpha}$, where $x_{1}, x_{2} \geq 0$ and $\sigma_{1}, \sigma_{2},(\alpha-1)>0$.

Marshall and Olkin (1995) considered distributions with limited memory, where the lack-ofmemory property holds true for a subset of the support of the distribution. With this weaker condition several classes of distributions were obtained, for example, bivariate exponential distributions with independent exponential marginals, the Marshall-Olkin distribution (2.4), bivariate exponential distributions with exponential scaled minima and bivariate exponential distributions with exponential minima.

Nair and Nair (1988a) proposed a definition of bivariate lack-of-memory property considering the two conditional expectations

$$
M_{i}\left(x_{1}, x_{2}\right)=\mathbb{E}\left(X_{i}-x_{i} \mid X_{1}>x_{1}, X_{2}>x_{2}\right), i=1,2,
$$

and imposing

$$
M_{i}\left(x_{1}, x_{2}\right)=\mathbb{E}\left(X_{i} \mid X_{3-i}>x_{3-i}\right), i=1,2 .
$$

for all $x_{1}, x_{2}>0$, characterizing Gumbel's Type I bivariate exponential distribution (2.1) as the only absolutely continuous distribution with this property.

In Roy (2004), the definition of bivariate lack-of-memory property also considers the two conditional expectations in (2.18) but imposes

$$
S_{X_{1}, X_{2}}\left(x_{1}+y_{1}, x_{2}\right) S_{X_{1}, X_{2}}\left(0, x_{2}\right)=S_{X_{1}, X_{2}}\left(x_{1}, x_{2}\right) S_{X_{1}, X_{2}}\left(y_{1} \frac{M_{1}\left(0, x_{2}\right)}{M_{1}\left(x_{1}, x_{2}\right)}, x_{2}\right)
$$

and

$$
S_{X_{1}, X_{2}}\left(x_{1}, x_{2}+y_{2}\right) S_{X_{1}, X_{2}}\left(x_{1}, 0\right)=S_{X_{1}, X_{2}}\left(x_{1}, x_{2}\right) S_{X_{1}, X_{2}}\left(x_{1}, y_{2} \frac{M_{2}\left(x_{1}, 0\right)}{M_{2}\left(x_{1}, x_{2}\right)}\right)
$$

for all $x_{1}, y_{1}, x_{2}, y_{2}>0$.

As a result, in case of dependence between $X_{1}$ and $X_{2}$, Roy (2004) characterized the Gumbel's Type I bivariate exponential distribution (2.1), the bivariate Lomax distribution with survival function $S_{X_{1}, X_{2}}\left(x_{1}, x_{2}\right)=\left\{\left(1+a_{1} x_{1}+a_{2} x_{2}+\gamma x_{1} x_{2}\right)\right\}^{-c}$, where $x_{1}, x_{2}>0, a_{1}, a_{2}, c>0$ and $0 \leq \gamma \leq(c+1) a_{1} a_{2}$. The bivariate finite range distribution due to Roy and Gupta (1996) with survival function $S_{X_{1}, X_{2}}\left(x_{1}, x_{2}\right)=\left\{\left(1-p_{1} x_{1}-p_{2} x_{2}-\delta x_{1} x_{2}\right)\right\}^{q}$, where $0<x_{1}<\frac{1}{p_{1}}, 0<x_{2}<\frac{1-p_{1} x_{1}}{p_{2}+\delta x_{1}}$, $p_{1}, p_{2}>0, p=\frac{2 k^{2}}{1-k^{2}}, \frac{1}{\sqrt{3}} \leq k \leq 1$, and $q-1 \geq \frac{\delta}{p_{1} p_{2}} \geq-1$ was also characterized. Sankaran (1992) also characterized these distributions, see Remark 5.4.1.

In case of independence between $X_{1}$ and $X_{2}$, their univariate distributions follow either the exponential distribution, the Lomax distribution with survival function $S_{X}(x)=\left(1+\frac{x}{a}\right)^{-\alpha}, x \geq 0$ and $a,(\alpha-1)>0$, or the finite range distribution $S_{X}(x)=\left(1-\frac{x}{a}\right)^{\alpha}, x \geq 0$ and $a, \alpha>0$. In the Lomax distribution, the restriction on the parameter $(\alpha-1)>0$ is necessary for the existence of 
the first moment $\mathbb{E}(X)$.

Chukova and Dimitrov (1992) introduced the distributions having the almost lack-of-memory property, defined by the relation

$$
\mathbb{P}(X-b \geq x \mid X \geq b)=\mathbb{P}(X \geq x)
$$

for all $x>0$ and infinitely many values of $b$. This property was extended to the bivariate case in Dimitrov et al. (1994), considering

$$
\mathbb{P}\left(X_{1} \geq a_{1}+x_{1}, X_{2} \geq a_{2}+x_{2} \mid X_{1} \geq a_{1}, X_{2} \geq a_{2}\right)=\mathbb{P}\left(X_{1} \geq x_{1}, X_{2} \geq x_{2}\right),
$$

for all $x_{1}, x_{2}>0$ and infinitely many different nonnegative values of $a_{1}$ and $a_{2}$. The corresponding survival copula is given in Dimitrov and Kolev (2013).

Remark 2.4.1 (Other definitions). If $M_{i}\left(x_{1}, x_{2}\right)$ in (2.18) are locally constants, i.e., $M_{i}\left(x_{1}, x_{2}\right)$ does not depend on $x_{i}, i=1,2$, then the bivariate lack-of-memory property defined in Roy (2002a) is obtained. This extension, along with the definition in Johnson and Kotz (1975) will be considered in Chapter 5. By its turn, the definition in Ghurye (1987) will be addressed in Chapter 6.

Remark 2.4.2 (Reversed lack-of-memory property). According to Asha and John (2009), let X be a random variable with support $(a, b)$, where $a<0$ and $b \geq 0$. Then $X$ is said to have the reversed lack-of-memory property if $\mathbb{P}(X \leq x \mid X \leq x+t)=\mathbb{P}(X \leq 0 \mid X \leq t)$, for all $a<x \leq x+t \leq b$. The property means that given a component has maximum lifetime at most $x+t$ then the failure of the component at any instant before $x+t$, say $x$, depends only on the residual time $t$ left, rather than $x$. This property was extended to the bivariate case and then generalized in John (2011). 


\section{Chapter 3}

\section{Extended Marshall-Olkin Model}

\subsection{Introduction}

Let us consider a vector $\mathbf{X}=\left(X_{1}, X_{2}\right)$ of nonnegative continuous random variables with joint survival function $S_{X_{1}, X_{2}}\left(x_{1}, x_{2}\right)=\mathbb{P}\left(X_{1}>x_{1}, X_{2}>x_{2}\right)$. As introduced in Chapter 2, recall that the vector $\left(X_{1}, X_{2}\right)$ meets the Marshall-Olkin model (to be abbreviated MO hereafter) whenever it admits the stochastic representation (2.9), i.e.,

$$
\left(X_{1}, X_{2}\right)=\left[\min \left(T_{1}, T_{3}\right), \min \left(T_{2}, T_{3}\right)\right]
$$

where the random variables $T_{i}$ are independent and exponentially distributed with positive parameters $\lambda_{i}, i=1,2,3$, respectively. Moreover, the bivariate survival function of $\left(X_{1}, X_{2}\right)$ is given by (2.4), i.e.,

$$
S_{X_{1}, X_{2}}\left(x_{1}, x_{2}\right)=\exp \left\{-\lambda_{1} x_{1}-\lambda_{2} x_{2}-\lambda_{3} \max \left(x_{1}, x_{2}\right)\right\} .
$$

The MO construction has a variety of applications. In reliability theory the MO model can describe the lifetime of a system of two components operating in a random environment and subject to three independent sources of damage, in actuarial science it can model the survival function of a married couple in a contract of joint life with last-survivor annuity while in theory of credit risk it can describe the times to default of two counterparties subject to three independent economic or financial unfavorable events.

To overcome the restriction of shocks and marginals exponentially distributed assumed in Marshall and Olkin (1967), Li and Pellerey (2011) proposed the Generalized Marshall-Olkin distribution (to be abbreviated GMO hereafter) assuming only independence among the nonnegative continuous random variables $T_{1}, T_{2}$ and $T_{3}$ in (2.9).

Let the lifetimes $T_{i}$ have survival function $S_{T_{i}}\left(x_{i}\right)=\mathbb{P}\left(T_{i}>x_{i}\right)$, density $f_{T_{i}}\left(x_{i}\right)$, failure rate $r_{T_{i}}\left(x_{i}\right)=\frac{f_{T_{i}}\left(x_{i}\right)}{S_{T_{i}}\left(x_{i}\right)}$ and denote by $H_{T_{i}}(x)=\int_{0}^{x} r_{T_{i}}(t) \mathrm{d} t$ their right continuous cumulative failure rate functions satisfying

$$
H_{T_{i}}(0)=0, H_{T_{i}}(\infty)=\infty \text { and } H_{T_{i}}\left(x_{i}\right)<\infty
$$

for $x_{i}>0, i=1,2,3$. Li and Pellerey (2011) show that in this case

$$
\begin{aligned}
S_{X_{1}, X_{2}}\left(x_{1}, x_{2}\right) & =S_{T_{1}}\left(x_{1}\right) S_{T_{2}}\left(x_{2}\right) S_{T_{3}}\left(\max \left(x_{1}, x_{2}\right)\right) \\
& =\exp \left\{-H_{T_{1}}\left(x_{1}\right)-H_{T_{2}}\left(x_{2}\right)-H_{T_{3}}\left(\max \left(x_{1}, x_{2}\right)\right)\right\} .
\end{aligned}
$$


Despite bringing more flexibility and enlarging the range of applications of MO distribution, the independence assumption in GMO model may not be found in the following situations:

1. two electric machines operating in the same factory and sharing the same maintenance policy, both subject to blackouts;

2. two components in the structure of the same building sharing reverse load or reverse set of stresses, both subject to earthquakes;

3. two different species living in the same environment and competing for food, both subject to floods and other natural catastrophes.

In the preceding examples, one can clearly identify dependence between $T_{1}$ and $T_{2}$ : it is positive in case 1 and negative in cases 2 and 3. The construction of a model that incorporates dependence between the random variables $T_{1}$ and $T_{2}$ and preserves the stochastic representation (2.9) is the core subject of this chapter.

The rest of this chapter is organized as follows. In Section 3.2 we describe our proposal for a possible extension of the MO and GMO models (2.4) and (3.1), and provide its basic properties. In Section 3.3 we derive the copula expression for the extended MO distribution, give related examples and study the distribution of the residual lifetimes. In Section 3.4 we offer two alternative copula representations. In Section 3.5 we present an absolutely continuous version of the extended MO model. Reliability properties are considered in Section 3.6, extreme value properties are addressed in Section 3.7 and a Bayesian data analysis is performed in Section 3.8. We finish with several comments, including admissible related research and applied directions.

\subsection{Extended Marshall-Olkin model and probabilistic properties}

Let us consider a pair $\left(T_{1}, T_{2}\right)$ of nonnegative continuous random variables defined by their joint survival function

$$
S_{T_{1}, T_{2}}\left(x_{1}, x_{2}\right)=\mathbb{P}\left(T_{1}>x_{1}, T_{2}>x_{2}\right) \quad \text { having marginals } S_{T_{1}}\left(x_{1}\right) \text { and } S_{T_{2}}\left(x_{2}\right) .
$$

In addition, assume that the random variable $T_{3}$ with survival function $S_{T_{3}}(x)=\mathbb{P}\left(T_{3}>x\right)$ is independent of $T_{1}$ and $T_{2}$. Hence, following the MO construction (2.9) we obtain the extended MO model (to be abbreviated EMO hereafter)

$$
S_{X_{1}, X_{2}}\left(x_{1}, x_{2}\right)=\mathbb{P}\left(T_{1}>x_{1}, T_{2}>x_{2}, T_{3}>\max \left\{x_{1}, x_{2}\right\}\right)=S_{T_{1}, T_{2}}\left(x_{1}, x_{2}\right) S_{T_{3}}\left(\max \left\{x_{1}, x_{2}\right\}\right) .
$$

Observe that GMO model (3.1) is a particular case of the model (3.2) when $T_{1}$ and $T_{2}$ are independent. If in addition the random variables $T_{1}, T_{2}$ and $T_{3}$ are exponentially distributed with parameters $\lambda_{1}, \lambda_{2}$ and $\lambda_{3}$, respectively, one gets the classical bivariate MO distribution (2.4). Figure 3.1 illustrates the relationship among the three models.

\subsubsection{Alternative representation for EMO model}

In order to investigate the properties of the EMO model, the exponential representation of a bivariate survival function turns out to be useful. Recall that any bivariate survival function can 


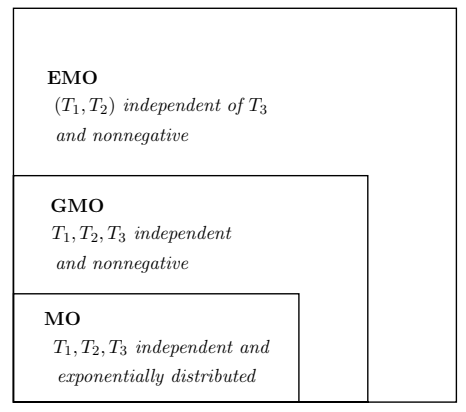

Figure 3.1: $M O, G M O$ and EMO models compared.

be represented by the relation

$$
S_{T_{1}, T_{2}}\left(x_{1}, x_{2}\right)=\exp \left\{-H_{T_{1}}\left(x_{1}\right)-H_{T_{2}}\left(x_{2}\right)+H_{T_{1}, T_{2}}\left(x_{1}, x_{2}\right)\right\}
$$

where the dependence function $H_{T_{1}, T_{2}}\left(x_{1}, x_{2}\right)$ is defined by

$$
H_{T_{1}, T_{2}}\left(x_{1}, x_{2}\right)=\ln \left\{\frac{S_{T_{1}, T_{2}}\left(x_{1}, x_{2}\right)}{S_{T_{1}}\left(x_{1}\right) S_{T_{2}}\left(x_{2}\right)}\right\}
$$

e.g., Finkelstein (2003). Representation (3.3) can be viewed as a generalization of the univariate exponential representation (i.e. $S_{T_{3}}(x)=\exp \left\{-H_{T_{3}}(x)\right\}$ for example), see Barlow and Proschan (1981).

It is worth noting that $H_{T_{1}, T_{2}}\left(0, x_{2}\right)=H_{T_{1}, T_{2}}\left(x_{1}, 0\right)=0$ for all $x_{1}, x_{2} \geq 0$. Moreover, $H_{T_{1}, T_{2}}\left(x_{1}, x_{2}\right)=$ 0 for all $x_{1}, x_{2} \geq 0$ characterizes independence between $T_{1}$ and $T_{2}$.

Under the above assumptions and notations, we define our Extended MO model in terms of cumulative failure rate and dependence functions as follows.

Definition 3.2.1. The Extended MO model is given by its survival function

$$
S_{X_{1}, X_{2}}\left(x_{1}, x_{2}\right)=\exp \left\{-H_{T_{1}}\left(x_{1}\right)-H_{T_{2}}\left(x_{2}\right)-H_{T_{3}}\left(\max \left(x_{1}, x_{2}\right)\right)+H_{T_{1}, T_{2}}\left(x_{1}, x_{2}\right)\right\} .
$$

Remark 3.2.2. Note that the only difference between GMO and EMO models is the presence of the dependence function $H_{T_{1}, T_{2}}\left(x_{1}, x_{2}\right)$ in (3.5), which has important features to understand the interaction between the random variables involved.

Remark 3.2.3. Whenever the joint distribution of the nonnegative random vector $\left(T_{1}, T_{2}\right)$ is absolutely continuous, with density function $f_{T_{1}, T_{2}}\left(x_{1}, x_{2}\right)$, following Finkelstein (2003), one can write

$$
H_{T_{1}, T_{2}}\left(x_{1}, x_{2}\right)=\int_{0}^{x_{1}} \int_{0}^{x_{2}}\left[r_{T_{1}, T_{2}}\left(y_{1}, y_{2}\right)-r_{1}\left(y_{1}, y_{2}\right) r_{2}\left(y_{1}, y_{2}\right)\right] \mathrm{d} y_{1} \mathrm{~d} y_{2}
$$

where $r_{T_{1}, T_{2}}\left(x_{1}, x_{2}\right)=\frac{f_{T_{1}, T_{2}}\left(x_{1}, x_{2}\right)}{S_{T_{1}, T_{2}}\left(x_{1}, x_{2}\right)}$ and $r_{i}\left(x_{1}, x_{2}\right)=-\frac{\partial}{\partial x_{i}} \ln \left[S_{T_{1}, T_{2}}\left(x_{1}, x_{2}\right)\right], i=1,2$, are, respectively, the bivariate failure rate, see Basu (1971), and the conditional hazard rates, see Johnson and Kotz (1975).

Remark 3.2.4. Denote $\Psi_{T_{1}, T_{2}}^{S}\left(x_{1}, x_{2}\right)=\frac{S_{T_{1}, T_{2}}\left(x_{1}, x_{2}\right)}{S_{T_{1}}\left(x_{1}\right) S_{T_{2}}\left(x_{2}\right)}$. Similarly to $H_{T_{1}, T_{2}}\left(x_{1}, x_{2}\right)$, the function $\Psi_{T_{1}, T_{2}}^{S}\left(x_{1}, x_{2}\right)$ characterizes the dependence structure between the random variables $T_{1}$ and $T_{2}$, see Remark 6.2.2. 
The next proposition shows two alternative representations for $\Psi_{T_{1}, T_{2}}^{S}\left(x_{1}, x_{2}\right)$.

Proposition 3.2.5. The function $\Psi_{T_{1}, T_{2}}^{S}\left(x_{1}, x_{2}\right)-1$ admits the following representations:

$$
\Psi_{T_{1}, T_{2}}^{S}\left(x_{1}, x_{2}\right)-1=\frac{\left[\mathbb{P}\left(T_{2}>x_{2} \mid T_{1}>x_{1}\right)-\mathbb{P}\left(T_{2}>x_{2} \mid T_{1} \leq x_{1}\right)\right]\left[1-\mathbb{P}\left(T_{1}>x_{1}\right)\right]}{\mathbb{P}\left(T_{2}>x_{2}\right)}
$$

and

$$
\Psi_{T_{1}, T_{2}}^{S}\left(x_{1}, x_{2}\right)-1=\frac{\left[\mathbb{P}\left(T_{1}>x_{1} \mid T_{2}>x_{2}\right)-\mathbb{P}\left(T_{1}>x_{1} \mid T_{2} \leq x_{2}\right)\right]\left[1-\mathbb{P}\left(T_{2}>x_{2}\right)\right]}{\mathbb{P}\left(T_{1}>x_{1}\right)} .
$$

Proof. Notice that $\mathbb{P}\left(T_{2}>x_{2}\right)$ can be written as

$$
\begin{aligned}
\mathbb{P}\left(T_{2}>x_{2}\right) & =\mathbb{P}\left(T_{1}>x_{1}, T_{2}>x_{2}\right)+\mathbb{P}\left(T_{1} \leq x_{1}, T_{2}>x_{2}\right) \\
& =\mathbb{P}\left(T_{1}>x_{1}, T_{2}>x_{2}\right)+\mathbb{P}\left(T_{2}>x_{2} \mid T_{1} \leq x_{1}\right) \mathbb{P}\left(T_{1} \leq x_{1}\right) .
\end{aligned}
$$

Multiplying both sides by $\mathbb{P}\left(T_{1}>x_{1}\right)$,

$$
\begin{aligned}
\mathbb{P}\left(T_{1}>x_{1}\right) \mathbb{P}\left(T_{2}>x_{2}\right) & =\mathbb{P}\left(T_{1}>x_{1}, T_{2}>x_{2}\right)\left[1-\mathbb{P}\left(T_{1} \leq x_{1}\right)\right] \\
& +\mathbb{P}\left(T_{2}>x_{2} \mid T_{1} \leq x_{1}\right) \mathbb{P}\left(T_{1} \leq x_{1}\right) \mathbb{P}\left(T_{1}>x_{1}\right) .
\end{aligned}
$$

The last expression can be also presented as

$$
\begin{aligned}
\mathbb{P}\left(T_{1}>x_{1}, T_{2}>x_{2}\right) & =\mathbb{P}\left(T_{1}>x_{1}\right) \mathbb{P}\left(T_{2}>x_{2}\right) \\
& +\left[\mathbb{P}\left(T_{2}>x_{2} \mid T_{1}>x_{1}\right)-\mathbb{P}\left(T_{2}>x_{2} \mid T_{1} \leq x_{1}\right)\right] \mathbb{P}\left(T_{1} \leq x_{1}\right) \mathbb{P}\left(T_{1}>x_{1}\right) .
\end{aligned}
$$

Similar result holds true considering $\mathbb{P}\left(T_{1}>x_{1}\right)$, finishing the proof.

Recall that a nonnegative random vector $\left(X_{1}, X_{2}\right)$ is positively quadrant dependent (PQD) if $\mathbb{P}\left(X_{1} \leq x_{1}, X_{2} \leq x_{2}\right) \geq \mathbb{P}\left(X_{1} \leq x_{1}\right) \mathbb{P}\left(X_{2} \leq x_{2}\right)$ for all $x_{1}, x_{2} \geq 0$, and negatively quadrant dependent $(\mathrm{NQD})$ when the last relation is valid with the inequality sign reversed, see Lehmann (1966).

Remark 3.2.6. From Proposition 3.2.5 it becomes clear the effect of a positive (or negative) quadrant dependence on the conditional probabilities $\mathbb{P}\left(T_{1}>x_{1} \mid T_{2}>x_{2}\right)$ and $\mathbb{P}\left(T_{1}>x_{1} \mid T_{2} \leq x_{2}\right)$. For example, let us suppose a positive quadrant dependence between $T_{1}$ and $T_{2}$. Then, for all $x_{1}, x_{2}$ we have $\mathbb{P}\left(T_{1}>x_{1} \mid T_{2} \leq x_{2}\right) \leq \mathbb{P}\left(T_{1}>x_{1}\right) \leq \mathbb{P}\left(T_{1}>x_{1} \mid T_{2}>x_{2}\right)$, what makes $\Psi^{S}\left(x_{1}, x_{2}\right)-1$ positive. Notice that the difference $\mathbb{P}\left(T_{1}>x_{1} \mid T_{2}>x_{2}\right)-\mathbb{P}\left(T_{1}>x_{1} \mid T_{2} \leq x_{2}\right)$ can be considered as a measure of local dependence.

\subsubsection{Probabilistic properties}

We will mention several probabilistic properties of the EMO model (3.5) formulated in Definition 3.2 .1 . 


\section{Positive and negative quadrant dependence}

The MO model (2.4) is always PQD, see relation (2.13), as well as the GMO model (3.1), see Li and Pellerey (2011). The EMO model (3.5) may be positively or negatively quadrant dependent, conditional on the distributional parameters involved. The following statement characterizes the NQD property of the EMO model.

Theorem 3.2.7. The vector $\left(X_{1}, X_{2}\right)$ following the EMO model is $N Q D$ if and only if

$$
S_{T_{1}, T_{2}}\left(x_{1}, x_{2}\right) \leq S_{T_{1}}\left(x_{1}\right) S_{T_{2}}\left(x_{2}\right) S_{T_{3}}\left(\min \left\{x_{1}, x_{2}\right\}\right)
$$

for all $x_{1}, x_{2} \geq 0$.

Proof. The necessary and sufficient condition given by relation (3.6) can be obtained from its equivalent inequality $S_{X_{1}, X_{2}}\left(x_{1}, x_{2}\right) \leq S_{X_{1}}\left(x_{1}\right) S_{X_{2}}\left(x_{2}\right)$

The following Corollary gives another version of the inequality (3.6) in terms of dependence function $H_{T_{1}, T_{2}}\left(x_{1}, x_{2}\right)$ given by (3.4) and the cumulative failure rate function $H_{T_{3}}(x)$.

Corollary 3.2.8. The vector $\left(X_{1}, X_{2}\right)$ following the EMO model is NQD if and only if

$$
H_{T_{1}, T_{2}}\left(x_{1}, x_{2}\right)+H_{T_{3}}\left(\min \left(x_{1}, x_{2}\right)\right) \leq 0
$$

for all $x_{1}, x_{2} \geq 0$.

Proof. Taking into account the exponential representation (3.5), one gets that the marginal survival functions have the following form in terms of cumulative failure rates: $S_{X_{i}}\left(x_{i}\right)=\exp \left\{-H_{T_{i}}\left(x_{i}\right)-\right.$ $\left.H_{T_{3}}\left(x_{i}\right)\right\}, i=1,2$. The vector $\left(X_{1}, X_{2}\right)$ is NQD if and only if $S_{X_{1}, X_{2}}\left(x_{1}, x_{2}\right) \leq S_{X_{1}}\left(x_{1}\right) S_{X_{2}}\left(x_{2}\right)$ for all $x_{1}, x_{2} \geq 0$. The last inequality is equivalent to

$$
\exp \left\{H_{T_{1}, T_{2}}\left(x_{1}, x_{2}\right)+H_{T_{3}}\left(\min \left(x_{1}, x_{2}\right)\right)\right\} \leq 1 .
$$

Taking logarithms on both sides we obtain the stated inequality.

Remark 3.2.9. Note that $X_{1}$ and $X_{2}$ defined by stochastic representation (2.9) are associated random variables since they are increasing functions of random variables $T_{1}, T_{2}$ and $T_{3}$. Hence, $X_{1}$ and $X_{2}$ are $P Q D$ in the MO and GMO models (2.4) and (3.1), according to Theorem 2.2 and Property $P_{3}$ given on page 30 in Barlow and Proschan (1981). Hence, the statement of Proposition 2.1 in Li and Pellerey (2011) follows without the need of obtaining the copula corresponding to the GMO distribution (3.1), nevertheless its usefulness. The same conclusion can not be handled for the EMO model since the vector $\left(T_{1}, T_{2}\right)$ may be NQD and not associated, therefore.

Before constructing an example of an EMO model which satisfies condition (3.6), we need the following result.

Theorem 3.2.10 (Nelsen (2006)). Let $C(u, v)$ be a copula. Then for every $(u, v) \in[0,1]^{2}$,

$$
\max (u+v-1,0) \leq C(u, v) \leq \min (u, v)
$$


Remark 3.2.11. Relation (3.7) is known as Fréchet-Hoeffding bounds inequality for copulas, since it holds true not only for bivariate copulas, but for bivariate distribution functions. The bounds in (3.7) are themselves copulas. The lower bound is commonly denoted by $W(u, v)=\max (u+v-1,0)$ whereas the upper bound, by $M(u, v)=\min (u, v)$. If the copula that joins two random variables $X_{1}$ and $X_{2}$ is $W(u, v)$, then the random variables are countermonotonic; but if the copula is $M(u, v)$, then $X_{1}$ and $X_{2}$ are comonotonic. Another important copula, given by $C(u, v)=u v$, is the independence copula, commonly used for performing quadrant dependence analyses, see Example 3.3.5.

Example 3.2.12. Let $T_{1}$ and $T_{2}$ be countermonotonic random variables, i.e., their joint distribution is given by the lower Fréchet bound, see Remark 3.2.11. Define

$$
\theta=\sup \left\{x \mid(x, x) \in \mathbf{W}\left(x_{1}, x_{2}\right)\right\}=\text { const }>0,
$$

where the set $\mathbf{W}\left(x_{1}, x_{2}\right) \subset[0, \infty)^{2}$ is given by

$$
\mathbf{W}\left(x_{1}, x_{2}\right)=\left\{\left(x_{1}, x_{2}\right) \in[0, \infty)^{2} \mid S_{T_{1}}\left(x_{1}\right)+S_{T_{2}}\left(x_{2}\right)>1\right\} .
$$

Define the distribution of $T_{3}$ as follows: $\mathbb{P}\left(T_{3}>\theta\right)=1$, i.e. the support of $T_{3}$ is the interval $(\theta, \infty)$. It is direct to check that specified distributions of $\left(T_{1}, T_{2}\right)$ and $T_{3}$ compose an EMO model which is $N Q D$.

Fixing the distributions, consider two unit exponential countermonotonic random variables $T_{1}$ and $T_{2}$. One may easy obtain that $\theta=\ln 2$ in this case, i.e. $\mathbb{P}\left(T_{3}>\ln 2\right)=1$. The dark area in Figure 3.2 represents the set $\mathbf{W}\left(x_{1}, x_{2}\right)$ for the specifications considered. We have

$$
S_{T_{1}, T_{2}}\left(x_{1}, x_{2}\right)<S_{T_{1}}\left(x_{1}\right) S_{T_{2}}\left(x_{2}\right) \quad \text { and } \quad S_{T_{3}}\left(\min \left(x_{1}, x_{2}\right)\right)=1
$$

if $\left(x_{1}, x_{2}\right) \in \mathbf{W}\left(x_{1}, x_{2}\right)$, i.e. relation (3.6) is fulfilled. Observe that $S_{T_{1}, T_{2}}\left(x_{1}, x_{2}\right)=0$ when $\left(x_{1}, x_{2}\right) \notin$ $\mathbf{W}\left(x_{1}, x_{2}\right)$. Therefore, the corresponding vector $\left(X_{1}, X_{2}\right)$ defined by (3.5) possesses the NQD property.

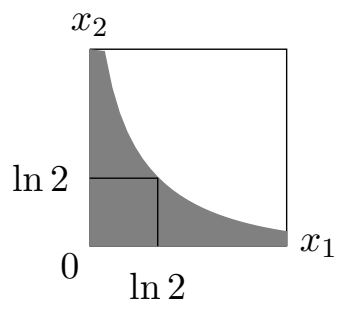

Figure 3.2: $\operatorname{Set} \mathbf{W}\left(x_{1}, x_{2}\right)$ in Example 3.2.12

Remark 3.2.13. It would be optimistic to expect in a real situation that NQD property of the vector $\left(X_{1}, X_{2}\right)$ would be valid for all observed pairs $\left(x_{1}^{*}, x_{2}^{*}\right)$ which should belong to the set $\mathbf{W}\left(x_{1}, x_{2}\right)$ defined in (3.8). In such cases, it is convenient to interpret "locally" the NQD property of $\left(X_{1}, X_{2}\right)$ when some pairs of observations are outside the set $\mathbf{W}\left(x_{1}, x_{2}\right)$, but the practical situation clearly indicates the use of NQD model. Example 3.3 .5 supports this conclusion in copula terms. 


\section{Symmetry, asymmetry and bounds for the joint survival function}

The MO model (2.4) may be exchangeable or not, depending on the parameters of the exponential distributions that $T_{1}$ and $T_{2}$ follow: if identically distributed then the MO model is symmetric. If one considers the GMO model (3.1) the same happens: if $T_{1}$ and $T_{2}$ are identically distributed then the GMO model is exchangeable. Both cases are analogous because the copula that joins $T_{1}$ and $T_{2}$ is the independence one, which is symmetric. Interestingly, in the EMO model (3.5), one may start with identically distributed random variables $T_{1}$ and $T_{2}$, but connected by a non exchangeable copula. Then the resulting EMO model will be non exchangeable. In fact, we proved the next statement.

Lemma 3.2.14. The EMO model is not necessarily exchangeable.

Example 3.2.15 (Non exchangeable EMO model with marginals identically distributed). Consider the stochastic representation (2.9) and let $T_{i}$ be exponentially distributed with parameter $\lambda_{i}=1$, i.e., $H_{T_{i}}\left(x_{i}\right)=x_{i}, i=1,2,3$. Then we have $X_{1}$ and $X_{2}$ exponentially distributed with a common parameter $\lambda=2$, i.e., $H_{X_{i}}\left(x_{i}\right)=2 x_{i}, i=1,2$. Denote $C_{T_{1}, T_{2}}(u, v)$ the copula that joins $\left(T_{1}, T_{2}\right)$ and consider Example 3.16 at page 84 in Nelsen (2006)

$$
C_{T_{1}, T_{2}}(u, v)=u v+u v(1-u)(1-v)[(a-b) v(1-u)+b]
$$

where $|b| \leq 1, \frac{b-3-\sqrt{9+6 b-3 b^{2}}}{2} \leq a \leq 1$ and $a \neq b$. From (2.14) the corresponding survival copula $\bar{C}_{T_{1}, T_{2}}(u, v)$ is

$$
\bar{C}_{T_{1}, T_{2}}(u, v)=u v\{1+(1-u)(1-v)[(a-b)(1-v) u+b]\} .
$$

Let $u=\exp \left\{-x_{1}\right\}$ and $v=\exp \left\{-x_{2}\right\}$ to obtain the joint survival function of $\left(T_{1}, T_{2}\right)$ as

$$
\begin{aligned}
S_{T_{1}, T_{2}}\left(x_{1}, x_{2}\right) & =\left[\exp \left\{-x_{1}-x_{2}\right\}\right] \\
& \times\left[1+\left(1-\exp \left\{-x_{1}\right\}\right)\left(1-\exp \left\{-x_{2}\right\}\right)\left[(a-b)\left(1-\exp \left\{-x_{2}\right\}\right) \exp \left\{-x_{1}\right\}+b\right]\right] .
\end{aligned}
$$

Therefore, the dependence function $H_{T_{1}, T_{2}}\left(x_{1}, x_{2}\right)$ defined by (3.4) is asymmetric

$$
H_{T_{1}, T_{2}}\left(x_{1}, x_{2}\right)=\ln \left[1+\left(1-\exp \left\{-x_{1}\right\}\right)\left(1-\exp \left\{-x_{2}\right\}\right)\left[(a-b)\left(1-\exp \left\{-x_{2}\right\}\right) \exp \left\{-x_{1}\right\}+b\right]\right] .
$$

As a result, $\left(X_{1}, X_{2}\right)=\left(\min \left(T_{1}, T_{3}\right), \min \left(T_{2}, T_{3}\right)\right)$ compose an EMO model with marginals identically distributed and asymmetric survival function given by (3.5),

$$
\begin{aligned}
S_{X_{1}, X_{2}}\left(x_{1}, x_{2}\right) & =\left[\exp \left\{-x_{1}-x_{2}-\max \left(x_{1}, x_{2}\right)\right\}\right] \\
& \times\left[1+\left(1-\exp \left\{-x_{1}\right\}\right)\left(1-\exp \left\{-x_{2}\right\}\right)\left[(a-b)\left(1-\exp \left\{-x_{2}\right\}\right) \exp \left\{-x_{1}\right\}+b\right]\right]
\end{aligned}
$$

Besides the knowledge of the marginal distributions of $T_{1}, T_{2}$ and $T_{3}$, a key aspect for deriving EMO models is the knowledge of joint distribution of $T_{1}$ and $T_{2}$ given by (3.3). An important component in (3.3) is the dependence function $H_{T_{1}, T_{2}}\left(t_{1}, t_{2}\right)$ representing the dependence structure between $T_{1}$ and $T_{2}$ free of the marginal influence. In the case of incomplete information, it is still possible to obtain bounds for the survival function of the EMO model (3.5) based on knowledge of the marginal survival functions $S_{T_{i}}$ or their cumulative failure rate functions $H_{T_{i}}, i=1,2,3$. The bounds for the survival function $S_{X_{1}, X_{2}}\left(x_{1}, x_{2}\right)$ of the EMO model are given below. 
Lemma 3.2.16. The lower and upper bounds for the survival function of the EMO model (3.5), are given by

$$
L\left(x_{1}, x_{2}\right) \leq S_{X_{1}, X_{2}}\left(x_{1}, x_{2}\right) \leq U\left(x_{1}, x_{2}\right)
$$

where

$$
L\left(x_{1}, x_{2}\right)=\max \left\{\left[\exp \left\{-H_{T_{1}}\left(x_{1}\right)\right\}+\exp \left\{-H_{T_{2}}\left(x_{2}\right)\right\}-1\right], 0\right\} \exp \left\{-H_{T_{3}}\left(\max \left(x_{1}, x_{2}\right)\right)\right\}
$$

and

$$
U\left(x_{1}, x_{2}\right)=\min \left\{\exp \left\{-H_{T_{1}}\left(x_{1}\right)\right\}, \exp \left\{-H_{T_{2}}\left(x_{2}\right)\right\}\right\} \exp \left\{-H_{T_{3}}\left(\max \left(x_{1}, x_{2}\right)\right)\right\} .
$$

Proof. Let a pair $\left(x_{1}, x_{2}\right)$ belongs to the set $\mathbf{W}\left(x_{1}, x_{2}\right)$ defined by (3.8), i.e., $S_{T_{1}}\left(x_{1}\right)+S_{T_{2}}\left(x_{2}\right)>1$. From Theorem 3.2.10, apply the usual Fréchet-Hoeffding bounds to the joint survival function of $\left(T_{1}, T_{2}\right)$ written in the exponential representation (3.3), to obtain the lower bound

$$
H_{T_{1}, T_{2}}\left(x_{1}, x_{2}\right) \geq H_{T_{1}}\left(x_{1}\right)+H_{T_{2}}\left(x_{2}\right)+\ln \left[\exp \left\{-H_{T_{1}}\left(x_{1}\right)\right\}+\exp \left\{-H_{T_{2}}\left(x_{2}\right)\right\}-1\right]
$$

and the upper bound

$$
H_{T_{1}, T_{2}}\left(x_{1}, x_{2}\right) \leq H_{T_{1}}\left(x_{1}\right)+H_{T_{2}}\left(x_{2}\right)+\ln \left\{\min \left[\exp \left\{-H_{T_{1}}\left(x_{1}\right)\right\}, \exp \left\{-H_{T_{2}}\left(x_{2}\right)\right\}\right]\right\}
$$

for all $\left(x_{1}, x_{2}\right) \in \mathbf{W}\left(x_{1}, x_{2}\right)$.

Substituting the above bounds in the expression of the survival function of EMO models (3.5), lower and upper bounds $L\left(x_{1}, x_{2}\right)$ and $U\left(x_{1}, x_{2}\right)$ for the joint survival function $S_{X_{1}, X_{2}}\left(x_{1}, x_{2}\right)$ stated by the Lemma will be obtained when $\left(x_{1}, x_{2}\right) \in \mathbf{W}\left(x_{1}, x_{2}\right)$. The case when $S_{T_{1}}\left(x_{1}\right)+S_{T_{2}}\left(x_{2}\right) \leq 1$ is trivial.

With additional information concerning the dependence structure between $\left(T_{1}, T_{2}\right)$ the bounds in Lemma 3.2.16 can be even sharpened, see Nelsen et al. (2001).

Remark 3.2.17. Whenever $\max \left(x_{1}, x_{2}\right)>0$ we have $L\left(x_{1}, x_{2}\right)>\max \left(S_{X_{1}}\left(x_{1}\right)+S_{X_{2}}\left(x_{2}\right)-1,0\right)$, as well as $U\left(x_{1}, x_{2}\right)<\min \left(S_{X_{1}}\left(x_{1}\right), S_{X_{2}}\left(x_{2}\right)\right)$, i.e., the bounds obtained in Lemma 3.2.16 are sharper than the well-known Fréchet-Hoeffding bounds given by (3.7) in Theorem 3.2.10.

\subsection{Survival copula representation and distributional property for residual lifetimes}

Firstly, we will obtain the survival copula $\bar{C}_{X_{1}, X_{2}}(u, v)$ corresponding to the joint survival function of the EMO model given by (3.5). Then, a distributional property of residual lifetimes vector will be established.

\subsubsection{Survival copula of the EMO model}

By Sklar's theorem, see Theorem 2.2.3 in Nelsen (2006), the dependence structure of a continuous bivariate random vector $\left(X_{1}, X_{2}\right)$ can be described by unique survival copula

$$
\bar{C}_{X_{1}, X_{2}}(u, v)=S_{X_{1}, X_{2}}\left(S_{X_{1}}^{-1}(u), S_{X_{2}}^{-1}(v)\right), \quad(u, v) \in[0,1],
$$


where $S_{X_{i}}^{-1}$ is the (generalized) inverse of $S_{X_{i}}, i=1,2$, see Theorem 2.2.3 in Nelsen (2006). The triplet $\left(S_{X_{1}}, S_{X_{2}}, \bar{C}_{X_{1}, X_{2}}\right)$ allows to analyse the dependence properties between $X_{1}$ and $X_{2}$.

Since we noted in the proof of the Corollary 3.2.8 that the marginal survival functions of $S_{X_{1}, X_{2}}\left(x_{1}, x_{2}\right)$ from (3.5) are given by $S_{X_{i}}\left(x_{i}\right)=\exp \left\{-H_{T_{i}}\left(x_{i}\right)-H_{T_{3}}\left(x_{i}\right)\right\}$, let us denote by $G_{i}\left(x_{i}\right)=H_{T_{i}}\left(x_{i}\right)+H_{T_{3}}\left(x_{i}\right), i=1,2$. Under the above notations, facts and relations, the following statement is true.

Lemma 3.3.1. The survival copula $\bar{C}_{X_{1}, X_{2}}(u, v)$ of the EMO model is given by

$$
\bar{C}_{X_{1}, X_{2}}(u, v)=\left\{\begin{array}{lll}
u v \exp \left\{H_{T_{3}}\left(G_{2}^{-1}(-\ln v)\right)+G(u, v)\right\}, & \text { if } \quad G_{1}^{-1}(-\ln u)>G_{2}^{-1}(-\ln v) \\
u v \exp \left\{H_{T_{3}}\left(G_{1}^{-1}(-\ln u)\right)+G(u, v)\right\}, & \text { if } \quad G_{1}^{-1}(-\ln u) \leq G_{2}^{-1}(-\ln v),
\end{array}\right.
$$

where $u, v \in(0,1)$ and $G(u, v)=H_{T_{1}, T_{2}}\left(S_{X_{1}}^{-1}(u), S_{X_{2}}^{-1}(v)\right)$.

Proof. Let $\bar{C}_{X_{1}, X_{2}}(u, v)$ be the survival copula corresponding to $S_{X_{1}, X_{2}}\left(x_{1}, x_{2}\right)$ from (3.5), i.e.

$$
\ln \left[\bar{C}_{X_{1}, X_{2}}(u, v)\right]=\ln \left[S_{X_{1}, X_{2}}\left(S_{X_{1}}^{-1}(u), S_{X_{2}}^{-1}(v)\right)\right], \quad u, v \in(0,1) .
$$

Observe that $S_{X_{1}}\left(x_{1}\right)=\exp \left\{-H_{T_{1}}\left(x_{1}\right)-H_{T_{3}}\left(x_{1}\right)\right\}=\exp \left\{-G_{1}\left(x_{1}\right)\right\}$. Because of absolute continuity of the distributions involved we have unique inverses. Solving $S_{X_{1}}\left(x_{1}\right)=u$ we get $S_{X_{1}}^{-1}(u)=x_{1}$. By analogy, from $\exp \left\{-G_{1}\left(x_{1}\right)\right\}=u$ we obtain $x_{1}=G_{1}^{-1}(-\ln u)$ and therefore

$$
S_{X_{1}}^{-1}(u)=G_{1}^{-1}(-\ln u), \quad \text { i.e. } \quad G_{1}\left(S_{X_{1}}^{-1}(u)\right)=-\ln u .
$$

In a similar way we obtain

$$
S_{X_{2}}^{-1}(v)=G_{2}^{-1}(-\ln v), \quad \text { i.e. } \quad G_{2}\left(S_{X_{2}}^{-1}(v)\right)=-\ln v .
$$

Let $x_{1}>x_{2} \geq 0$, i.e. $S_{X_{1}}^{-1}(u)>S_{X_{2}}^{-1}(v)$, or equivalently $G_{1}^{-1}(-\ln u)>G_{2}^{-1}(-\ln v)$. Using relation (3.5) we have

$$
\ln \left[\bar{C}_{X_{1}, X_{2}}(u, v)\right]=\ln \left[\exp \left\{-H_{T_{1}}\left(S_{X_{1}}^{-1}(u)\right)-H_{T_{2}}\left(S_{X_{2}}^{-1}(v)\right)-H_{T_{3}}\left(S_{X_{1}}^{-1}(u)\right)+G(u, v)\right\}\right],
$$

with $G(u, v)=H_{T_{1}, T_{2}}\left(S_{X_{1}}^{-1}(u), S_{X_{2}}^{-1}(v)\right)$, which is equivalent to

$$
\ln \left[\bar{C}_{X_{1}, X_{2}}(u, v)\right]=\ln u-H_{T_{2}}\left(S_{X_{2}}^{-1}(v)\right)+G(u, v) .
$$

Due to (3.11) we get

$$
\ln \bar{C}_{X_{1}, X_{2}}(u, v)=\ln u v+H_{T_{3}}\left(S_{X_{2}}^{-1}(v)\right)+G(u, v) .
$$

Finally, $\bar{C}_{X_{1}, X_{2}}(u, v)=u v \exp \left\{H_{T_{3}}\left(G_{2}^{-1}(-\ln v)\right)+G(u, v)\right\}$ if $S_{X_{1}}^{-1}(u)>S_{X_{2}}^{-1}(v)$.

By analogy, when $0 \leq x_{1} \leq x_{2}$, i.e. $G_{1}^{-1}(-\ln u) \leq G_{2}^{-1}(-\ln v)$, one obtains

$$
\bar{C}_{X_{1}, X_{2}}(u, v)=u v \exp \left\{H_{T_{3}}\left(G_{2}^{-1}(-\ln v)\right)+G(u, v)\right\}
$$

and representation (3.9) is established. 
Remark 3.3.2. The function $\exp \{G(u, v)\}$ is the only product extra multiplier in (3.9) in addition to the copula expression corresponding to GMO distribution and numbered (2.3) by Li and Pellerey (2011). This extra term permits $N Q D$ modelling on $\left(X_{1}, X_{2}\right)$, as shown in Example 3.3.5.

Remark 3.3.3. Whenever $\left(T_{1}, T_{2}\right)$ is absolutely continuous, using Remark 3.2.3, we have the alternative representation for $G(u, v)$ given by

$$
G(u, v)=\int_{0}^{S_{X_{1}}^{-1}(u)} \int_{0}^{S_{X_{2}}^{-1}(v)}\left[r_{T_{1}, T_{2}}\left(y_{1}, y_{2}\right)-r_{1}\left(y_{1}, y_{2}\right) r_{2}\left(y_{1}, y_{2}\right)\right] \mathrm{d} y_{1} \mathrm{~d} y_{2} .
$$

The following example illustrates how the survival copula given in (3.9) can be obtained in a particular case.

Example 3.3.4 (Copula of the EMO model). Consider $T_{i}$ exponentially distributed with parameter $\lambda_{i}>0, i=1,2$, and joined by the Gumbel survival copula (family B6 in Joe (1997)), also known as Gumbel-Hourgaard copula (see equation (2.30) in Balakrishnan and Lai (2009)), so that

$$
S_{T_{1}, T_{2}}\left(x_{1}, x_{2}\right)=\exp \left\{-\left[\left(\lambda_{1} x_{1}\right)^{\theta}+\left(\lambda_{2} x_{2}\right)^{\theta}\right]^{\frac{1}{\theta}}\right\}
$$

where $\theta \geq 1$, and let $T_{3}$ be independent of $\left(T_{1}, T_{2}\right)$ with survival function $S_{T_{3}}(x)=\exp \left(-\lambda_{3} x\right)$, $\lambda_{3}>0$.

Following the above specifications, we have $H_{T_{i}}(x)=\lambda_{i} x, i=1,2,3$. It is easy to check that $G_{i}(x)=\left(\lambda_{i}+\lambda_{3}\right) x, G_{i}^{-1}(u)=\frac{u}{\lambda_{i}+\lambda_{3}}$ and $S_{X_{i}}^{-1}(u)=-\frac{\ln u}{\lambda_{i}+\lambda_{3}}$, for $i=1,2$. By its turn,

$$
H_{T_{1}, T_{2}}\left(x_{1}, x_{2}\right)=\ln \frac{S_{T_{1}, T_{2}}\left(x_{1}, x_{2}\right)}{S_{T_{1}}\left(x_{1}\right) S_{T_{2}}\left(x_{2}\right)}=-\left[\left(\lambda_{1} x_{1}\right)^{\theta}+\left(\lambda_{2} x_{2}\right)^{\theta}\right]^{\frac{1}{\theta}}+\lambda_{1} x_{1}+\lambda_{2} x_{2} .
$$

Thus we have

$$
G(u, v)=H_{T_{1}, T_{2}}\left(S_{X_{1}}^{-1}(u), S_{X_{2}}^{-1}(v)\right)=-\left[\left(\ln u^{-\frac{\lambda_{1}}{\lambda_{1}+\lambda_{3}}}\right)^{\theta}+\left(\ln v^{-\frac{\lambda_{2}}{\lambda_{2}+\lambda_{3}}}\right)^{\theta}\right]^{\frac{1}{\theta}}-\ln u^{\frac{\lambda_{1}}{\lambda_{1}+\lambda_{3}}} v^{\frac{\lambda_{2}}{\lambda_{2}+\lambda_{3}}} .
$$

Substituting the relations in (3.9) we get the survival copula

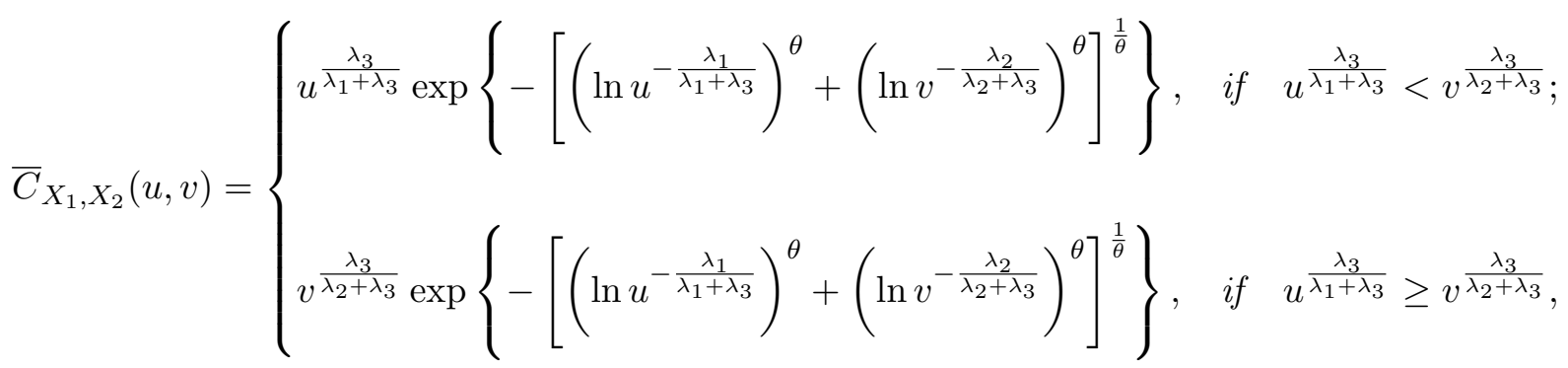

where $u, v \in(0,1]$. Notice that when $\theta=1$ we obtain the MO copula, see relation (2.15).

In the next example we show that the same EMO survival function is locally PQD and locally NQD, in the spirit of Remarks 3.2.13 and 3.3.2 (depending on parameters range).

Example 3.3.5 (Copula of the EMO model and quadrant dependence analysis). Let $\left(T_{1}, T_{2}\right)$ follow the Gumbel's type I bivariate distribution with unit exponential marginals and corresponding survival 
function given by (2.1), i.e.,

$$
S_{T_{1}, T_{2}}\left(x_{1}, x_{2}\right)=\exp \left(-x_{1}-x_{2}-\theta x_{1} x_{2}\right)
$$

$x_{1}, x_{2} \geq 0$ and $\theta \in[0,1]$, and let $T_{3}$ be independent of $\left(T_{1}, T_{2}\right)$ with survival function $S_{T_{3}}(x)=$ $\exp (-\lambda x), \lambda>0$. Following the above notations, we have $H_{T_{1}}(x)=H_{T_{2}}(x)=x, H_{T_{3}}(x)=\lambda x$ and $H_{T_{1}, T_{2}}\left(x_{1}, x_{2}\right)=-\theta x_{1} x_{2}$. Therefore,

$$
G_{1}\left(x_{1}\right)=H_{T_{1}}\left(x_{1}\right)+H_{T_{3}}\left(x_{1}\right)=(1+\lambda) x_{1} \quad \text { and } \quad G_{2}\left(x_{2}\right)=H_{T_{2}}\left(x_{2}\right)+H_{T_{3}}\left(x_{2}\right)=(1+\lambda) x_{2} .
$$

Their inverse functions are given by

$$
G_{1}^{-1}(u)=\frac{u}{1+\lambda} \quad \text { and } \quad G_{2}^{-1}(v)=\frac{v}{1+\lambda} .
$$

Since $\ln (x)$ is an increasing function and $1+\lambda>0$, we have the following set of equivalent inequalities:

$$
G_{1}^{-1}(-\ln (u))>G_{2}^{-1}(-\ln (v)) \Leftrightarrow \frac{-\ln (u)}{1+\lambda}>\frac{-\ln (v)}{1+\lambda} \Leftrightarrow \frac{\ln (u)}{1+\lambda}<\frac{\ln (v)}{1+\lambda} \Leftrightarrow 0<u<v \leq 1 .
$$

Since $G(u, v)=H_{T_{1}, T_{2}}\left(G_{1}^{-1}(-\ln (u)), G_{2}^{-1}(-\ln (v))\right)=-\theta \frac{\ln (u) \ln (v)}{(1+\lambda)^{2}}$, we obtain the survival copula for the EMO model

$$
\bar{C}_{X_{1}, X_{2}}(u, v)= \begin{cases}u v \exp \left\{-\lambda \frac{\ln (v)}{1+\lambda}-\theta \frac{\ln (u) \ln (v)}{(1+\lambda)^{2}}\right\}, & \text { if } 0<u<v \leq 1 ; \\ u v \exp \left\{-\lambda \frac{\ln (u)}{1+\lambda}-\theta \frac{\ln (u) \ln (v)}{(1+\lambda)^{2}}\right\}, & \text { if } 1 \geq u \geq v>0 .\end{cases}
$$

When $0<u<v \leq 1$, we have $\bar{C}_{X_{1}, X_{2}}(u, v)<u v$ if

$$
\exp \left\{-\lambda \frac{\ln (v)}{1+\lambda}-\theta \frac{\ln (u) \ln (v)}{(1+\lambda)^{2}}\right\} \leq 1
$$

This inequality is equivalent to

$$
0<u \leq u_{0}=\exp \left\{-\frac{\lambda(1+\lambda)}{\theta}\right\}
$$

By analogy, when $0<v \leq u \leq 1$ and $0<v \leq v_{0}=\exp \left\{-\frac{\lambda(1+\lambda)}{\theta}\right\}$ we get $\bar{C}_{X_{1}, X_{2}}(u, v) \leq u v$.

Therefore, as illustrated in Figure 3.3, when $(u, v) \in\left[\exp \left\{-\frac{\lambda(1+\lambda)}{\theta}\right\}, 1\right]^{2}$ we have the local PQD property and outside this set, the local NQD property.

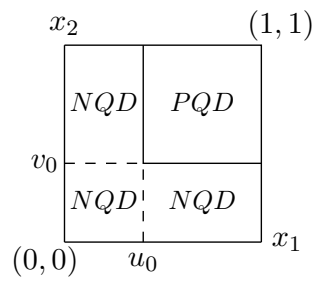

Figure 3.3: Dependence analysis of the EMO model in Example 3.3.5. 


\subsubsection{Distributional property for residual lifetimes}

Many authors have studied the monotonicity of the conditional probability

$$
\mathbb{P}\left(X_{1}>x_{1}+t, X_{2}>x_{2}+t \mid X_{1}>t, X_{2}>t\right)
$$

in $x_{1}, x_{2} \geq 0$ for all $t \geq 0$, which is the joint survival function of the random vector

$$
\mathbf{X}_{t}=\left[\left(X_{1}-t, X_{2}-t\right) \mid X_{1}>t, X_{2}>t\right]
$$

being the pair of residual lifetimes at time $t \geq 0$. The problem has important and innumerous applications in industry, medicine, finance, economics, insurance, see Singpurwalla (2006) and McNeil et al. (2005).

The aging performance of the residual lifetime vector $\mathbf{X}_{t}$ is well studied when the dependence structure between $X_{1}$ and $X_{2}$ is described by the family of Archimedean copulas, for details see Bassan and Spizzichino (2005) and Mulero and Pellerey (2010). Thus, the results obtained are valid for exchangeable random vectors, i.e. for the class of bivariate distributions with same marginal distributions, which is a limitation for practical needs and applications.

The GMO type distributions given by (3.1) do not possess the bivariate lack-of-memory property (2.6) and for this reason Li and Pellerey (2011) investigated the aging behaviour and dependence properties of $\mathbf{X}_{t}$. In particular, they show that if $\mathbf{X}$ has a GMO distribution, then so does $\mathbf{X}_{t}$.

We are now interested to examine the property of the residual lifetime vector $\mathbf{X}_{t}$ under the EMO model (3.5). The result is given in the following theorem.

Theorem 3.3.6. If the vector $\left(X_{1}, X_{2}\right)$ follows an EMO model, then so does $\mathbf{X}_{t}$ for any $t \geq 0$.

Proof. The survival function of the residual lifetime vector (3.13) is

$$
\begin{aligned}
\mathbb{P}\left(\mathbf{X}_{t}>\left(x_{1}, x_{2}\right)\right) & =\mathbb{P}\left(X_{1}-t>x_{1}, X_{2}-t>x_{2} \mid X_{1}>t, X_{2}>t\right) \\
& =\mathbb{P}\left(\min \left\{T_{1}-t, T_{3}-t\right\}>x_{1}, \min \left\{T_{2}-t, T_{3}-t\right\}>x_{2} \mid \min \left\{T_{1}, T_{3}\right\}>t, \min \left\{T_{2}, T_{3}\right\}>t\right) .
\end{aligned}
$$

Taking into account that in the EMO model the random variable $T_{3}$ is independent of the vector $\left(T_{1}, T_{2}\right)$ we get

$$
\mathbb{P}\left(\mathbf{X}_{t}>\left(x_{1}, x_{2}\right)\right)=\frac{\mathbb{P}\left(T_{1}>t+x_{1}, T_{2}>t+x_{2}\right) \mathbb{P}\left(T_{3}>t+\max \left\{x_{1}, x_{2}\right\}\right)}{\mathbb{P}\left(T_{1}>t, T_{2}>t\right) \mathbb{P}\left(T_{3}>t\right)},
$$

i.e.

$$
\mathbb{P}\left(\mathbf{X}_{t}>\left(x_{1}, x_{2}\right)\right)=\frac{S_{T_{1}, T_{2}}\left(t+x_{1}, t+x_{2}\right)}{S_{T_{1}, T_{2}}(t, t)} \times \frac{S_{T_{3}}\left(t+\max \left\{x_{1}, x_{2}\right\}\right)}{S_{T_{3}}(t)} .
$$

The last relation means that

$$
\mathbb{P}\left(\mathbf{X}_{t}>\left(x_{1}, x_{2}\right)\right)=\mathbb{P}\left(T_{1, t}>x_{1}, T_{2, t}>x_{2}\right) \mathbb{P}\left(T_{3, t}>\max \left\{x_{1}, x_{2}\right\}\right)
$$

where $T_{i, t}=\left[T_{i}-t \mid T_{1}>t, T_{2}>t\right], i=1,2$, and $T_{3, t}=\left[T_{3}-t \mid T_{3}>t\right]$. Thus, for any $t \geq 0$ the residual lifetime vector $\mathbf{X}_{t}$ in the EMO model has a stochastic representation

$$
\mathbf{X}_{t}=\left[\min \left\{T_{1, t}, T_{3, t}\right\}, \min \left\{T_{2, t} T_{3, t}\right\}\right],
$$


e.g. $\mathbf{X}_{t}$ has the same form stochastic representation (2.9). This means that if the random vector $\left(X_{1}, X_{2}\right)$ follows an EMO model with survival function given by (3.5) then the corresponding residual lifetime vector $\mathbf{X}_{t}$ also follows an EMO model.

As a consequence of the Theorem 6.B.16(b) in Shaked and Shanthikumar (2007), it follows that $\left(X_{1}, X_{2}\right)$ and $\mathbf{X}_{t}$ have the same type of copula (i.e., given by relation (3.9)) even if they are generated by different triples of random variables.

Remark 3.3.7. If $T_{3}$ is exponentially distributed and $\left(T_{1}, T_{2}\right)$ possesses the bivariate lack-ofmemory property, i.e., $S_{T_{1}, T_{2}}\left(x_{1}+t, x_{2}+t\right)=S_{T_{1}, T_{2}}\left(x_{1}, x_{2}\right) S_{T_{1}, T_{2}}(t, t)$ for all $x_{1}, x_{2}, t>0$, e.g., the Block and Basu exponential distribution, see Block and Basu (19\%4), then $\left(X_{1}, X_{2}\right)$ will exhibit the bivariate lack-of-memory property, so that $\mathbf{X}_{t}$ and $\mathbf{X}$ will have the same distribution and copula, which are independent of $t$. See also related Theorem 6.2.16.

\subsection{Other survival copula representations}

In this section we obtain an alternative copula representation of the EMO model as a function of conditional hazard rates $r_{i}\left(x_{1}, x_{2}\right), i=1,2$. After that we will present an inverse copula relation. Several typical examples will be presented.

\subsubsection{Alternative survival copula representation}

Let us denote $\Lambda_{T_{1}, T_{2}}\left(x_{1}, x_{2}\right)=-\ln S_{T_{1}, T_{2}}\left(x_{1}, x_{2}\right)$. Then the exponential representation (3.3) implies the relation

$$
\Lambda_{T_{1}, T_{2}}\left(x_{1}, x_{2}\right)=H_{T_{1}}\left(x_{1}\right)+H_{T_{2}}\left(x_{2}\right)-H_{T_{1}, T_{2}}\left(x_{1}, x_{2}\right) .
$$

It is direct to check that

$$
\Lambda_{T_{1}, T_{2}}\left(x_{1}, 0\right)=-\ln \left(S_{T_{1}}\left(x_{1}\right)\right)=H_{T_{1}}\left(x_{1}\right)
$$

and

$$
\Lambda_{T_{1}, T_{2}}\left(0, x_{2}\right)=-\ln \left(S_{T_{2}}\left(x_{2}\right)\right)=H_{T_{2}}\left(x_{2}\right) .
$$

For the conditional hazard rates, let us consider $S_{T_{1}, T_{2}}\left(x_{1}, x_{2}\right)$ absolutely continuous, so we have

$$
r_{i}\left(x_{1}, x_{2}\right)=-\frac{\partial}{\partial x_{i}} \ln \left[S_{T_{1}, T_{2}}\left(x_{1}, x_{2}\right)\right]=\frac{\partial}{\partial x_{i}} \Lambda_{T_{1}, T_{2}}\left(x_{1}, x_{2}\right), i=1,2 .
$$

By definition, $r_{1}\left(x_{1}, x_{2}\right)=\frac{\partial}{\partial x_{1}} \Lambda_{T_{1}, T_{2}}\left(x_{1}, x_{2}\right)$, i.e., $\int_{0}^{x_{1}} r_{1}\left(z, x_{2}\right) \mathrm{d} z=\int_{0}^{x_{1}} \frac{\partial}{\partial x_{1}} \Lambda_{T_{1}, T_{2}}\left(z, x_{2}\right) d z$, which implies the relation

$$
\Lambda_{T_{1}, T_{2}}\left(x_{1}, x_{2}\right)=\int_{0}^{x_{1}} r_{1}\left(z, x_{2}\right) \mathrm{d} z+H_{T_{2}}\left(x_{2}\right)
$$

Likewise

$$
\Lambda_{T_{1}, T_{2}}\left(x_{1}, x_{2}\right)=\int_{0}^{x_{2}} r_{2}\left(x_{1}, z\right) \mathrm{d} z+H_{T_{1}}\left(x_{1}\right)
$$


Since $S_{T_{1}, T_{2}}\left(x_{1}, x_{2}\right)=\exp \left\{-\Lambda_{T_{1}, T_{2}}\left(x_{1}, x_{2}\right)\right\}$, from the expression for the survival function of EMO models (3.2) we have

$$
S_{X_{1}, X_{2}}\left(x_{1}, x_{2}\right)=S_{T_{1}, T_{2}}\left(x_{1}, x_{2}\right) S_{T_{3}}\left(\max \left(x_{1}, x_{2}\right)\right)=\exp \left\{-\Lambda_{T_{1}, T_{2}}\left(x_{1}, x_{2}\right)\right\} S_{T_{3}}\left(\max \left(x_{1}, x_{2}\right)\right) .
$$

Using (3.14) we obtain

$$
S_{X_{1}, X_{2}}\left(x_{1}, x_{2}\right)=\exp \left\{-\int_{0}^{x_{1}} r_{1}\left(z, x_{2}\right) \mathrm{d} z-H_{T_{2}}\left(x_{2}\right)-H_{T_{3}}\left(\max \left(x_{1}, x_{2}\right)\right)\right\} .
$$

Remind that $G_{2}\left(x_{2}\right)=H_{T_{2}}\left(x_{2}\right)+H_{T_{3}}\left(x_{2}\right)$ and assume $x_{2}>x_{1} \geq 0$. Then

$$
S_{X_{1}, X_{2}}\left(x_{1}, x_{2}\right)=\exp \left\{-\int_{0}^{x_{1}} r_{1}\left(z, x_{2}\right) \mathrm{d} z-G_{2}\left(x_{2}\right)\right\} .
$$

If $\bar{C}_{X_{1}, X_{2}}(u, v)$ is the survival copula corresponding to $S_{X_{1}, X_{2}}\left(x_{1}, x_{2}\right)$, we have

$$
\ln \left[\bar{C}_{X_{1}, X_{2}}(u, v)\right]=\ln \left[\exp \left\{-\int_{0}^{S_{X_{1}}^{-1}(u)} r_{1}\left(z, S_{X_{2}}^{-1}(v)\right) \mathrm{d} z-G_{2}\left(S_{X_{2}}^{-1}(v)\right)\right\}\right] .
$$

Using (3.16) we obtain the copula expression when $x_{2}>x_{1}$, i.e., $S_{X_{2}}^{-1}(v)>S_{X_{1}}^{-1}(u)$

$$
\bar{C}_{X_{1}, X_{2}}(u, v)=v \exp \left\{-\int_{0}^{S_{X_{1}}^{-1}(u)} r_{1}\left(z, S_{X_{2}}^{-1}(v)\right) \mathrm{d} z\right\} .
$$

Using the same steps, one can get the corresponding expression when $x_{1} \geq x_{2} \geq 0$, i.e., when $S_{X_{1}}^{-1}(u) \geq S_{X_{2}}^{-1}(v)$. In fact, we have the following theorem.

Theorem 3.4.1. Let $S_{T_{1}, T_{2}}\left(x_{1}, x_{2}\right)$ be absolutely continuous. Then the survival copula $\bar{C}_{X_{1}, X_{2}}(u, v)$ of the EMO model (3.2) is given by

$$
\bar{C}_{X_{1}, X_{2}}(u, v)= \begin{cases}v \exp \left\{-\int_{0}^{S_{X_{1}}^{-1}(u)} r_{1}\left(z, S_{X_{2}}^{-1}(v)\right) \mathrm{d} z\right\}, & \text { if } \quad S_{X_{1}}^{-1}(u)<S_{X_{2}}^{-1}(v) ; \\ u \exp \left\{-\int_{0}^{S_{X_{2}}^{-1}(v)} r_{2}\left(S_{X_{1}}^{-1}(u), z\right) \mathrm{d} z\right\}, & \text { if } \quad S_{X_{1}}^{-1}(u) \geq S_{X_{2}}^{-1}(v),\end{cases}
$$

where $u, v \in[0,1]$.

The NQD property will be satisfied if $\int_{0}^{S_{X_{1}}^{-1}(u)} r_{1}\left(z, S_{X_{2}}^{-1}(v)\right) \mathrm{d} z>-\ln u=G_{1}\left(S_{X_{1}}^{-1}(u)\right)$ when $S_{X_{1}}^{-1}(u)<S_{X_{2}}^{-1}(v)$, and if $\int_{0}^{S_{X_{2}}^{-1}(v)} r_{2}\left(S_{X_{1}}^{-1}(u), z\right) \mathrm{d} z>-\ln v=G_{2}\left(S_{X_{2}}^{-1}(v)\right)$ when $S_{X_{1}}^{-1}(u) \geq S_{X_{2}}^{-1}(v)$, depending on the parameter values.

\subsubsection{Inverse survival copula representation}

The representation (3.9) gives the survival copula $\bar{C}_{X_{1}, X_{2}}(u, v)$ of the EMO model as a function of joint distribution of $\left(T_{1}, T_{2}\right)$ and $T_{3}$. In some occasions, one may be interested on inverse situation: to obtain the survival copula $\bar{C}_{T_{1}, T_{2}}(u, v)$ representing the dependence structure between $T_{1}$ and $T_{2}$ in the EMO model under the knowledge of the distribution of $T_{3}$ and joint distribution of $X_{1}$ and $X_{2}$. The next theorem provides this relation. 
Theorem 3.4.2. Let $\left(X_{1}, X_{2}\right)$ be a bivariate random vector that follows the EMO model (3.2) generated by the random variables $\left(T_{1}, T_{2}, T_{3}\right)$. If $S_{X_{1}, X_{2}}\left(x_{1}, x_{2}\right)$ and $S_{T_{3}}(x)$ are the survival functions of $\left(X_{1}, X_{2}\right)$ and $T_{3}$, respectively, then the survival copula of the bivariate random vector $\left(T_{1}, T_{2}\right)$ is

$$
\bar{C}_{T_{1}, T_{2}}(u, v)=\frac{S_{X_{1}, X_{2}}\left\{H_{T_{1}}^{-1}(-\ln u), H_{T_{2}}^{-1}(-\ln v)\right\}}{S_{T_{3}}\left\{\max \left(H_{T_{1}}^{-1}(-\ln u), H_{T_{2}}^{-1}(-\ln v)\right)\right\}},
$$

where $H_{T_{1}}\left(x_{1}\right)=\ln \left(\frac{S_{T_{3}}\left(x_{1}\right)}{S_{X_{1}, X_{2}}\left(x_{1}, 0\right)}\right), H_{T_{2}}\left(x_{2}\right)=\ln \left(\frac{S_{T_{3}}\left(x_{2}\right)}{S_{X_{1}, X_{2}}\left(0, x_{2}\right)}\right)$ and $(u, v) \in(0,1]^{2}$.

Proof. From the exponential representation for the survival function of $T_{3}$, we have $H_{T_{3}}(x)=$ $-\ln \left(S_{T_{3}}(x)\right)$. From the expression for survival function of EMO models (3.2) we obtain

$$
S_{T_{1}, T_{2}}\left(x_{1}, x_{2}\right)=\frac{S_{X_{1}, X_{2}}\left(x_{1}, x_{2}\right)}{S_{T_{3}}\left(\max \left(x_{1}, x_{2}\right)\right)}
$$

Set $x_{2}=0$ in the previous equation and use the expression for $H_{T_{3}}(x)$ to obtain

$$
H_{T_{1}}\left(x_{1}\right)=-\ln \left\{S_{X_{1}, X_{2}}\left(x_{1}, 0\right)\right\}-H_{T_{3}}\left(x_{1}\right)=\ln \left(\frac{S_{T_{3}}\left(x_{1}\right)}{S_{X_{1}, X_{2}}\left(x_{1}, 0\right)}\right) .
$$

From the exponential representation for bivariate survival functions (3.3), set $x_{2}=0$ to obtain the survival function of $T_{1}$, i.e., $S_{T_{1}}\left(x_{1}\right)$, and fix $S_{T_{1}}\left(x_{1}\right)=u$,

$$
S_{T_{1}}\left(x_{1}\right)=\exp \left\{-H_{T_{1}}\left(x_{1}\right)\right\}=u \Rightarrow x_{1}=S_{T_{1}}^{-1}(u)=H_{T_{1}}^{-1}(-\ln u)
$$

Analogously, $H_{T_{2}}\left(x_{2}\right)=\ln \left(\frac{S_{T_{3}}\left(x_{2}\right)}{S_{X_{1}, X_{2}}\left(0, x_{2}\right)}\right)$ and $x_{2}=S_{T_{2}}^{-1}(v)=H_{T_{2}}^{-1}(-\ln v)$. Since $\bar{C}_{T_{1}, T_{2}}(u, v)=$ $S_{T_{1}, T_{2}}\left(S_{T_{1}}^{-1}(u), S_{T_{2}}^{-1}(v)\right)$, the result follows after the corresponding substitutions.

We apply Theorem 3.4.2 in the next four examples.

Example 3.4.3 (Lomax survival copula). Consider the EMO model with survival function

$$
S_{X_{1}, X_{2}}\left(x_{1}, x_{2}\right)=\left\{\left(1+a_{1} x_{1}+a_{2} x_{2}+\gamma x_{1} x_{2}\right)\left(1+a_{3} \max \left(x_{1}, x_{2}\right)\right)\right\}^{-c},
$$

where $x_{1}, x_{2}>0, a_{1}, a_{2}, a_{3}, c>0$ and $0 \leq \gamma \leq(c+1) a_{1} a_{2}$. Moreover, let $T_{3}$ follow a Pareto type II distribution, with location parameter equal to $0, S_{T_{3}}(x)=\left(1+a_{3} x\right)^{-c}$, where $x>0$. From Theorem 3.4.2, $S_{T_{1}, T_{2}}\left(x_{1}, x_{2}\right)=\left\{\left(1+a_{1} x_{1}+a_{2} x_{2}+\gamma x_{1} x_{2}\right)\right\}^{-c}$ and $H_{T_{i}}\left(x_{i}\right)=c \ln \left(1+a_{i} x_{i}\right), i=1$, 2. Thus, $H_{T_{1}}^{-1}(-\ln u)=\frac{u^{-\frac{1}{c}}-1}{a_{1}}$ and $H_{T_{2}}^{-1}(-\ln v)=\frac{v^{-\frac{1}{c}}-1}{a_{2}}$. After the corresponding substitutions, we obtain the survival copula for $\left(T_{1}, T_{2}\right)$ as

$$
\bar{C}_{T_{1}, T_{2}}(u, v)=\frac{u v}{\left\{1-\left(1-\frac{\gamma}{a_{1} a_{2}}\right)\left(1-u^{\frac{1}{c}}\right)\left(1-v^{\frac{1}{c}}\right)\right\}^{c}},
$$

where $u, v \in(0,1], a_{1}, a_{2}, c>0$ and $0 \leq \gamma \leq(c+1) a_{1} a_{2}$. This is the Lomax survival copula, see equation (2.41) in Balakrishnan and Lai (2009).

Example 3.4.4. Consider the EMO model with survival function

$$
S_{X_{1}, X_{2}}\left(x_{1}, x_{2}\right)=\left[\left(1-p_{1} x_{1}-p_{2} x_{2}\right) \exp \left\{-\max \left(x_{1}, x_{2}\right)\right\}\right]^{c},
$$


where $0<x_{1}<\frac{1}{p_{1}}, 0<x_{2}<\frac{1-p_{1} x_{1}}{p_{2}}, p_{1}, p_{2}>0$ and $c>1$. Moreover, suppose $T_{3}$ follows a Weibull distribution, $S_{T_{3}}(x)=\exp \left\{-x^{c}\right\}$, where $x>0$. From Theorem 3.4.2, $S_{T_{1}, T_{2}}\left(x_{1}, x_{2}\right)=\left(1-p_{1} x_{1}-\right.$ $\left.p_{2} x_{2}\right)^{c}$ and $H_{T_{i}}\left(x_{i}\right)=-c \ln \left(1-p_{i} x_{i}\right), i=1,2$. Thus, $H_{T_{1}}^{-1}(-\ln u)=\frac{1-u^{\frac{1}{c}}}{p_{1}}$ and $H_{T_{2}}^{-1}(-\ln v)=\frac{1-v^{\frac{1}{c}}}{p_{2}}$. After the corresponding substitutions, we obtain the survival copula for $\left(T_{1}, T_{2}\right)$ as

$$
\bar{C}_{T_{1}, T_{2}}(u, v)=\left(u^{\frac{1}{c}}+v^{\frac{1}{c}}-1\right)^{c},
$$

where $u, v \in(0,1], u^{\frac{1}{c}}+v^{\frac{1}{c}}-1>0$ and $c>1$. This is the survival copula associated with the multivariate rescaled Dirichlet distribution, see Ma (1996), when considering the bivariate case.

Example 3.4.5 (Gumbel-Barnett survival copula). Consider the EMO model with survival function:

$$
S_{X_{1}, X_{2}}\left(x_{1}, x_{2}\right)=\exp \left\{-\lambda_{1} x_{1}-\lambda_{2} x_{2}-\lambda_{3} \max \left(x_{1}, x_{2}\right)-\theta \lambda_{1} \lambda_{2} x_{1} x_{2}\right\},
$$

where $x_{1}, x_{2}>0, \lambda_{i}>0, i=1,2,3$, and $0 \leq \theta \leq 1$. Moreover, $T_{3}$ follows an exponential distribution, $S_{T_{3}}(x)=\exp \left\{-\lambda_{3} x\right\}$, where $x>0$. From Theorem 3.4.2, $S_{T_{1}, T_{2}}\left(x_{1}, x_{2}\right)=\exp \left\{-\lambda_{1} x_{1}-\lambda_{2} x_{2}-\theta \lambda_{1} \lambda_{2} x_{1} x_{2}\right\}$ and $H_{T_{i}}\left(x_{i}\right)=-\lambda_{i} x_{i}, i=1,2$. Thus, $H_{T_{1}}^{-1}(-\ln u)=-\frac{\ln u}{\lambda_{1}}$ and $H_{T_{2}}^{-1}(-\ln v)=-\frac{\ln v}{\lambda_{2}}$. After the corresponding substitutions, we obtain the survival copula for $\left(T_{1}, T_{2}\right)$ as

$$
\bar{C}_{T_{1}, T_{2}}(u, v)=u v \exp \{-\theta \ln u \ln v\}
$$

where $u, v \in(0,1]$ and $0 \leq \theta \leq 1$. This survival copula is known as Gumbel-Barnett, see equation (2.49) in Balakrishnan and Lai (2009).

Example 3.4.6 (Pareto (or Clayton) survival copula). Consider the EMO model with survival function:

$$
S_{X_{1}, X_{2}}\left(x_{1}, x_{2}\right)= \begin{cases}\exp \left\{-\lambda_{3} \max \left(x_{1}, x_{2}\right)\right\}, & \text { if } 0 \leq x_{1}<\theta_{1} \text { and } 0 \leq x_{2}<\theta_{2}, \\ \left(\frac{\theta_{1}}{x_{1}}\right)^{a} \exp \left\{-\lambda_{3} \max \left(x_{1}, x_{2}\right)\right\}, & \text { if } x_{1} \geq \theta_{1} \text { and } 0 \leq x_{2}<\theta_{2}, \\ \left(\frac{\theta_{2}}{x_{2}}\right)^{a} \exp \left\{-\lambda_{3} \max \left(x_{1}, x_{2}\right)\right\}, & \text { if } 0 \leq x_{1}<\theta_{1} \text { and } x_{2} \geq \theta_{2}, \\ \frac{\left(\theta_{1} \theta_{2}\right)^{a}}{\left(\theta_{2} x_{1}+\theta_{1} x_{2}-\theta_{1} \theta_{2}\right)^{a}} \exp \left\{-\lambda_{3} \max \left(x_{1}, x_{2}\right)\right\}, & \text { if } x_{1} \geq \theta_{1}, x_{2} \geq \theta_{2},\end{cases}
$$

where $\lambda_{3}>0$ and $a>0$. Moreover, it is known that $T_{3}$ follows an exponential distribution, $S_{T_{3}}(x)=\exp \left\{-\lambda_{3} x\right\}$, where $x \geq 0$. From Theorem 3.4.2, $S_{T_{1}, T_{2}}\left(x_{1}, x_{2}\right)=\frac{\left(\theta_{1} \theta_{2}\right)^{a}}{\left(\theta_{2} x_{1}+\theta_{1} x_{2}-\theta_{1} \theta_{2}\right)^{a}}, x_{i} \geq \theta_{i}$, and $H_{T_{i}}\left(x_{i}\right)=a \ln \left[\frac{x_{i}}{\theta_{i}}\right], i=1,2$. Thus, $H_{T_{1}}^{-1}(-\ln u)=\frac{\theta_{1}}{\sqrt[a]{u}}$ and $H_{T_{2}}^{-1}(-\ln v)=\frac{\theta_{2}}{\sqrt[a]{v}}$. After the corresponding substitutions, we obtain the survival copula for $\left(T_{1}, T_{2}\right)$ as

$$
\bar{C}_{T_{1}, T_{2}}(u, v)=\left(u^{-\frac{1}{a}}+v^{-\frac{1}{a}}-1\right)^{-a},
$$

where $u, v \in(0,1]$ and $a>0$. This copula is known as Pareto (or Clayton) survival copula, see equation (2.43) in Balakrishnan and Lai (2009). 


\subsection{An absolutely continuous version of EMO model}

If the bivariate random vector $\left(X_{1}, X_{2}\right)$ satisfies the EMO model (3.5), its survival function has no discrete component and from the Lebesgue decomposition (2.11) we have

$$
S_{X_{1}, X_{2}}\left(x_{1}, x_{2}\right)=(1-\alpha) S_{X_{1}, X_{2}}^{a c}\left(x_{1}, x_{2}\right)+\alpha S_{X_{1}, X_{2}}^{s i}\left(\max \left\{x_{1}, x_{2}\right\}\right)
$$

where $S_{X_{1}, X_{2}}^{a c}\left(x_{1}, x_{2}\right)$ is an absolutely continuous survival function, $S_{X_{1}, X_{2}}^{s i}\left(\max \left\{x_{1}, x_{2}\right\}\right)$ is the singular component with support on the set $\Omega$ given by $(2.10)$ and $\alpha \in[0,1]$.

Notice that $\alpha=\mathbb{P}\left(X_{1}=X_{2}\right)$, so if $\alpha=0$ then the joint distribution of the random vector $\left(X_{1}, X_{2}\right)$ is absolutely continuous. Denoting by

$$
(1-\alpha) f_{X_{1}, X_{2}}^{a c}\left(x_{1}, x_{2}\right)=\frac{\partial^{2}}{\partial x_{1} \partial x_{2}} S_{X_{1}, X_{2}}\left(x_{1}, x_{2}\right),
$$

the value of $\alpha$ can be obtained integrating both sides of (3.19)

$$
1-\alpha=\int_{0}^{\infty} \int_{0}^{\infty} \frac{\partial^{2}}{\partial x_{1} \partial x_{2}} S_{X_{1}, X_{2}}\left(x_{1}, x_{2}\right) \mathrm{d} x_{1} \mathrm{~d} x_{2} .
$$

By its turn, the survival function of EMO models (3.2) can be decomposed in terms of the survival functions of $\left(T_{1}, T_{2}\right)$ and $T_{3}$ as

$$
S_{X_{1}, X_{2}}\left(x_{1}, x_{2}\right)= \begin{cases}S_{T_{1}, T_{2}}\left(x_{1}, x_{2}\right) S_{T_{3}}\left(x_{1}\right), & \text { if } x_{1} \geq x_{2} \geq 0 \\ S_{T_{1}, T_{2}}\left(x_{1}, x_{2}\right) S_{T_{3}}\left(x_{2}\right), & \text { if } 0 \leq x_{1}<x_{2}\end{cases}
$$

so from (3.19)

$(1-\alpha) f_{X_{1}, X_{2}}^{a c}\left(x_{1}, x_{2}\right)= \begin{cases}S_{T_{3}}\left(x_{1}\right) \frac{\partial^{2}}{\partial x_{1} \partial x_{2}} S_{T_{1}, T_{2}}\left(x_{1}, x_{2}\right)+\frac{\partial}{\partial x_{2}} S_{T_{1}, T_{2}}\left(x_{1}, x_{2}\right) \frac{d}{d x_{1}} S_{T_{3}}\left(x_{1}\right), & \text { if } x_{1} \geq x_{2} \geq 0, \\ S_{T_{3}}\left(x_{2}\right) \frac{\partial^{2}}{\partial x_{1} \partial x_{2}} S_{T_{1}, T_{2}}\left(x_{1}, x_{2}\right)+\frac{\partial}{\partial x_{1}} S_{T_{1}, T_{2}}\left(x_{1}, x_{2}\right) \frac{d}{d x_{2}} S_{T_{3}}\left(x_{2}\right), & \text { if } 0 \leq x_{1}<x_{2},\end{cases}$

which is calculated where the derivatives exist, and depends on the densities of $\left(T_{1}, T_{2}\right)$ and $T_{3}$. Therefore, a necessary condition for $S_{X_{1}, X_{2}}\left(x_{1}, x_{2}\right)$ to be absolutely continuous is the absolute continuity of the distributions of $\left(T_{1}, T_{2}\right)$ and $T_{3}$.

The singular component in (3.20) with support on the set $\Omega$ can be analysed using Theorem 1.1 of Joe (1997), which is stated in terms of distribution functions, but remains valid if one works with survival functions.

Theorem 3.5.1 (Joe (1997)). Let $F_{X_{1}, X_{2}}\left(x_{1}, x_{2}\right)$ be a continuous bivariate distribution with univariate margins $F_{X_{1}}\left(x_{1}\right), F_{X_{2}}\left(x_{2}\right)$ and conditional distribution $F_{X_{2} \mid X_{1}}\left(x_{2} \mid x_{1}\right)$ of the second variable given the first. Suppose that $F_{X_{2} \mid X_{1}}\left(x_{2} \mid x_{1}\right)$ has jump discontinuities totalling a mass of $a\left(x_{1}\right)$, and $a\left(x_{1}\right)$ is continuous and positive on an interval. Then $F_{X_{1}, X_{2}}\left(x_{1}, x_{2}\right)$ has a singular component and the mass of the singular component is $\int_{0}^{\infty} a(u) \mathrm{d} F_{X_{1}}(u)$. A similar result holds for the conditional distribution $F_{X_{1} \mid X_{2}}\left(x_{1} \mid x_{2}\right)$ of the first variable given the second.

Therefore, let us denote by $S_{X_{2} \mid X_{1}}\left(x_{2} \mid x_{1}\right)$ the conditional survival function of $X_{2}$ given $X_{1}=x_{1}$,

$$
S_{X_{2} \mid X_{1}}\left(x_{2} \mid x_{1}\right)=\frac{-\frac{\partial}{\partial x_{1}} S_{X_{1}, X_{2}}\left(x_{1}, x_{2}\right)}{-\frac{d}{d x_{1}} S_{X_{1}}\left(x_{1}\right)} .
$$


In order to obtain the jump discontinuities of $S_{X_{2} \mid X_{1}}\left(x_{2} \mid x_{1}\right)$ at the points $x_{1}=x_{2}=x \geq 0$ we first calculate

$$
S_{X_{2} \mid X_{1}}\left(x_{2} \mid x_{1}\right)= \begin{cases}\frac{S_{T_{3}}\left(x_{1}\right) \frac{\partial}{\partial x_{1}} S_{T_{1}, T_{2}}\left(x_{1}, x_{2}\right)+S_{T_{1}, T_{2}}\left(x_{1}, x_{2}\right) \frac{d}{d x_{1}} S_{T_{3}}\left(x_{1}\right)}{d x_{1}} S_{X_{1}}\left(x_{1}\right) & \text { if } x_{1} \geq x_{2}, \\ \frac{S_{T_{3}}\left(x_{2}\right) \frac{\partial}{\partial x_{1}} S_{T_{1}, T_{2}}\left(x_{1}, x_{2}\right)}{\frac{d}{d x_{1}} S_{X_{1}}\left(x_{1}\right)}, & \text { if } x_{1}<x_{2} .\end{cases}
$$

When $x_{1}=x_{2}=x \geq 0$, the jump discontinuities have mass $a(x)=-\frac{S_{T_{1}, T_{2}}(x, x) f_{T_{3}}(x)}{\frac{d}{d x} S_{X_{1}}(x)}$, and the total mass of the singular component is given by:

$$
\alpha=\int_{x=0}^{\infty}-a(x) \mathrm{d} S_{X_{1}}(x)=\int_{x=0}^{\infty} S_{T_{1}, T_{2}}(x, x) f_{T_{3}}(x) \mathrm{d} x .
$$

Therefore, in order to remove this singularity, we have to impose

$$
S_{T_{1}, T_{2}}(x, x) f_{T_{3}}(x)=0 \text { for all } x \geq 0 .
$$

The same conclusion is obtained when analysing the jump discontinuities of $S_{X_{1} \mid X_{2}}\left(x_{1} \mid x_{2}\right)$ at the points $x_{1}=x_{2}=x \geq 0$.

Notice that if $S_{T_{1}, T_{2}}(x, x)>0$ for all finite and nonnegative $x$, then we have to impose $f_{T_{3}}(x)=0$, $x \geq 0$. This last condition resembles Claims 4.5 and 4.6 in Singpurwalla (2006) when analysing the discontinuity in MO model.

As an interpretation of relation (3.21), define the random variable $T=\min \left(T_{1}, T_{2}\right)$, with survival function $S_{T}(x)=S_{T_{1}, T_{2}}(x, x)$ : the EMO model does not possess a singular component whenever $\alpha=\mathbb{P}\left(X_{1}=X_{2}\right)=0$, which happens if and only if $\mathbb{P}\left(T_{3}<T\right)=0$.

The singular component in EMO model is removed after imposing three conditions:

1. The support of the random variable $T$ is bounded from above. Let us denote $\theta=\sup \left\{x \geq 0: S_{T_{1}, T_{2}}(x, x)>0\right\}$

2. the support of the random variable $T_{3}$ is bounded from below. Let us denote $\gamma=\inf \left\{x \geq 0: S_{T_{3}}(x)<1\right\}$

3. the following relation holds true: $\gamma \geq \theta$.

Remark 3.5.2. Notice that the first condition implies $S_{T_{1}, T_{2}}\left(x_{1}, x_{2}\right)=0$ whenever $x_{1}, x_{2}>\theta$, since any survival function is non-increasing in both arguments. Moreover, if the supports of the marginal distributions of the bivariate random vector $\left(T_{1}, T_{2}\right)$ are not bounded from above then $H_{T_{1}}(\theta)$ and $H_{T_{2}}(\theta)$ are finite. From the exponential representation for bivariate survival functions (3.3) we can conclude that

$$
H_{T_{1}, T_{2}}(\theta, \theta)=\int_{0}^{\theta} \int_{0}^{\theta}\left(r_{T_{1}, T_{2}}\left(y_{1}, y_{2}\right)-r_{1}\left(y_{1}, y_{2}\right) r_{2}\left(y_{1}, y_{2}\right)\right) \mathrm{d} y_{1} \mathrm{~d} y_{2}=-\infty,
$$

meaning that at least one of the conditional hazard rates of $\left(T_{1}, T_{2}\right)$ is unbounded from above. 
Therefore, the survival function of the EMO model under the above assumptions is

$$
S_{X_{1}, X_{2}}\left(x_{1}, x_{2}\right)= \begin{cases}S_{T_{1}, T_{2}}\left(x_{1}, x_{2}\right), & \text { if } 0 \leq x_{1}, x_{2}<\gamma \\ S_{T_{1}, T_{2}}\left(x_{1}, x_{2}\right) S_{T_{3}}\left(x_{1}\right), & \text { if } x_{1} \geq \min \left(x_{2}, \gamma\right), \\ S_{T_{1}, T_{2}}\left(x_{1}, x_{2}\right) S_{T_{3}}\left(x_{2}\right), & \text { if } x_{2} \geq \min \left(x_{1}, \gamma\right) .\end{cases}
$$

Thus, we have proved the following Theorem:

Theorem 3.5.3. Suppose $\left(T_{1}, T_{2}\right)$ and $T_{3}$ are nonnegative absolutely continuous random variables, with $\left(T_{1}, T_{2}\right)$ independent of $T_{3}$. Let $\theta=\sup \left\{x \geq 0: S_{T_{1}, T_{2}}(x, x)>0\right\}$ and $\gamma=\inf \{x \geq 0$ : $\left.S_{T_{3}}(x)<1\right\}$. If $\theta \leq \gamma<\infty$ then $\left(X_{1}, X_{2}\right)=\left(\min \left(T_{1}, T_{3}\right), \min \left(T_{2}, T_{3}\right)\right)$ compose an absolutely continuous EMO model with survival function given by (3.22).

Example 3.5.4 (Absolutely continuous EMO model). Consider the survival copula $\bar{C}_{T_{1}, T_{2}}(u, v)=$ $\left(u^{\frac{1}{c}}+v^{\frac{1}{c}}-1\right)^{c}$, where $u, v \in(0,1], u^{\frac{1}{c}}+v^{\frac{1}{c}}-1>0$ and $c>1$, obtained in Example 3.4.4. Fix unit exponential marginals for $T_{1}$ and $T_{2}$ to obtain

$$
S_{T_{1}, T_{2}}\left(x_{1}, x_{2}\right)=\left(\exp \left\{-\frac{x_{1}}{c}\right\}+\exp \left\{-\frac{x_{2}}{c}\right\}-1\right)^{c}
$$

$\left(x_{1}, x_{2}\right) \in \mathbf{D}$, where $\mathbf{D}=\left\{\left(x_{1}, x_{2}\right): x_{1}>0, x_{2}>0, \exp \left\{-\frac{x_{1}}{c}\right\}+\exp \left\{-\frac{x_{2}}{c}\right\}-1>0\right\}$. Following Theorem 3.5.3, for such survival function, $\theta=c \ln 2$. Fix $\gamma=\theta$ and consider $T_{3}$ independent of $\left(T_{1}, T_{2}\right)$, with survival function $S_{T_{3}}(x)=\exp \{-(x-c \ln 2)\}, x \geq c \ln 2$. Therefore, according with Theorem 3.5.3, $\left(T_{1}, T_{2}\right)$ and $T_{3}$ compose an absolutely continuous EMO model with survival function:

$$
\begin{aligned}
& S_{X_{1}, X_{2}}\left(x_{1}, x_{2}\right)= \\
& = \begin{cases}\left(\exp \left\{-\frac{x_{1}}{c}\right\}+\exp \left\{-\frac{x_{2}}{c}\right)-1\right\}^{c}, & \text { if }\left(x_{1}, x_{2}\right) \in \mathbf{D}, x_{1}, x_{2}<c \ln 2 ; \\
\left(\exp \left\{-\frac{x_{1}}{c}\right\}+\exp \left\{-\frac{x_{2}}{c}\right\}-1\right)^{c} \exp \left\{-\left(x_{1}-c \ln 2\right)\right\}, & \text { if }\left(x_{1}, x_{2}\right) \in \mathbf{D}, x_{1} \geq \min \left(x_{2}, c \ln 2\right) ; \\
\left(\exp \left\{-\frac{x_{1}}{c}\right\}+\exp \left\{-\frac{x_{2}}{c}\right\}-1\right)^{c} \exp \left\{-\left(x_{2}-c \ln 2\right)\right\}, & \text { if }\left(x_{1}, x_{2}\right) \in \mathbf{D}, x_{2} \geq \min \left(x_{1}, c \ln 2\right) .\end{cases}
\end{aligned}
$$

Moreover, we have $\alpha=\mathbb{P}\left(X_{1}=X_{2}\right)=0$ and the density function $f_{X_{1}, X_{2}}\left(x_{1}, x_{2}\right)$ can be obtained from (3.19) as

$$
\begin{aligned}
& f_{X_{1}, X_{2}}\left(x_{1}, x_{2}\right)=\frac{\partial^{2}}{\partial x_{1} \partial x_{2}} S_{X_{1}, X_{2}}\left(x_{1}, x_{2}\right)= \\
& = \begin{cases}\frac{c-1}{c} \exp \left\{-\frac{x_{1}+x_{2}}{c}\right\}\left(\exp \left\{-\frac{x_{1}}{c}\right\}+\exp \left\{-\frac{x_{2}}{c}\right\}-1\right)^{c-2}, & \text { if }\left(x_{1}, x_{2}\right) \in \mathbf{D}, x_{1}, x_{2}<c \ln 2 ; \\
\exp \left\{-x_{1}-\frac{x_{2}}{c}+c \ln 2\right\}\left(\exp \left\{-\frac{x_{1}}{c}\right\}+\exp \left\{-\frac{x_{2}}{c}\right\}-1\right)^{c-2} & \\
\times\left(\frac{2 c-1}{c} \exp \left\{-\frac{x_{1}}{c}\right\}+\exp \left\{-\frac{x_{2}}{c}\right\}-1\right), & \text { if }\left(x_{1}, x_{2}\right) \in \mathbf{D}, x_{1} \geq \min \left(x_{2}, c \ln 2\right) ; \\
\exp \left\{-x_{2}-\frac{x_{1}}{c}+c \ln 2\right\}\left(\exp \left\{-\frac{x_{1}}{c}\right\}+\exp \left\{-\frac{x_{2}}{c}\right\}-1\right)^{c-2} & \\
\times\left(\frac{2 c-1}{c} \exp \left\{-\frac{x_{2}}{c}\right\}+\exp \left\{-\frac{x_{1}}{c}\right\}-1\right), & \text { if }\left(x_{1}, x_{2}\right) \in \mathbf{D}, x_{2} \geq \min \left(x_{1}, c \ln 2\right) .\end{cases}
\end{aligned}
$$




\subsection{Reliability properties}

In this section we consider the bivariate lack-of-memory property (LMP) in EMO models. In the sequel, we analyse their weak bivariate increasing failure rate as well as bivariate increasing hazard rate properties.

\subsubsection{Bivariate lack-of-memory property}

We can obtain several examples of EMO models given by (3.5) that display the bivariate lackof-memory property. Consider the following

Example 3.6.1 (EMO model with bivariate LMP). Consider three nonnegative absolutely continuous and independent random variables $Y_{i}, i=1,2,3$, and let $S_{Y_{1}}(x)=S_{Y_{2}}(x)=\exp \{-x\}$. For the failure rate of $Y_{3}$, fix $r_{Y_{3}}(x)=1+\sin (x)$ so that it has cumulative failure rate $H_{Y_{3}}(x)=\int_{0}^{x}[1+$ $\sin (u)] \mathrm{d} u=x-\cos (x)+1$. Consider the GMO distribution $\left(T_{1}, T_{2}\right)=\left(\min \left(Y_{1}, Y_{3}\right), \min \left(Y_{2}, Y_{3}\right)\right)$ with survival function $S_{T_{1}, T_{2}}\left(x_{1}, x_{2}\right)=\exp \left\{-x_{1}-x_{2}-H_{Y_{3}}\left(\max \left(x_{1}, x_{2}\right)\right)\right\}$. Now, let the nonnegative absolutely continuous random variable $T_{3}$ be independent of $\left(T_{1}, T_{2}\right)$ with failure rate $r_{T_{3}}(x)=1-\sin (x)$ and cumulative failure rate $H_{T_{3}}(x)=\int_{0}^{x}[1-\sin (u)] \mathrm{d} u=x+\cos (x)-1$. Let us compose the EMO model $\left(X_{1}, X_{2}\right)=\left(\min \left(T_{1}, T_{3}\right), \min \left(T_{2}, T_{3}\right)\right)$. From the expression for survival function of EMO models (3.2) we have $S_{X_{1}, X_{2}}\left(x_{1}, x_{2}\right)=S_{T_{1}, T_{2}}\left(x_{1}, x_{2}\right) S_{T_{3}}\left(\max \left(x_{1}, x_{2}\right)\right)$. Therefore, $S_{X_{1}, X_{2}}\left(x_{1}, x_{2}\right)=$ $\exp \left\{-x_{1}-x_{2}-H_{Y_{3}}\left(\max \left(x_{1}, x_{2}\right)\right)-H_{T_{3}}\left(\max \left(x_{1}, x_{2}\right)\right)\right\}=\exp \left\{-x_{1}-x_{2}-2 \max \left(x_{1}, x_{2}\right)\right\}$, i.e. $\left(X_{1}, X_{2}\right)$ follows a Marshall-Olkin distribution (2.4) which displays the bivariate LMP according with relation (2.6). Notice that $S_{T_{1}, T_{2}}\left(x_{1}, x_{2}\right)$ does not exhibit the bivariate $L M P$, is not absolutely continuous nor $T_{3}$ is exponentially distributed. This distribution will also be considered in Example 3.7.9.

Remark 3.6.2. In Example 3.6.1, $S_{T_{1}, T_{2}}\left(x_{1}, x_{2}\right)$ exhibits a singular component with support on the set $\Omega=\left\{\left(x_{1}, x_{2}\right) \mid x_{1}=x_{2}=x \geq 0\right\}$. With additional conditions on the joint distribution of $\left(T_{1}, T_{2}\right)$ the bivariate LMP in EMO models can be uniquely characterized, see Theorem 3.6.6 and Corollary 3.6.8.

Let $\left(T_{1}, T_{2}\right)$ display the bivariate LMP and let $T_{3}$ follow an exponential distribution. Therefore, the EMO model constructed with these random variables possesses the bivariate LMP, since from the expression for survival function of EMO models (3.2) and the LMP of $T_{3}$,

$$
\begin{aligned}
S_{X_{1}, X_{2}}\left(x_{1}+t, x_{2}+t\right) & =S_{T_{1}, T_{2}}\left(x_{1}+t, x_{2}+t\right) S_{T_{3}}\left(\max \left(x_{1}+t, x_{2}+t\right)\right) \\
& =S_{T_{1}, T_{2}}\left(x_{1}+t, x_{2}+t\right) S_{T_{3}}\left(\max \left(x_{1}, x_{2}\right)+t\right) \\
& =S_{T_{1}, T_{2}}\left(x_{1}, x_{2}\right) S_{T_{3}}\left(\max \left(x_{1}, x_{2}\right)\right) S_{T_{1}, T_{2}}(t, t) S_{T_{3}}(t) .
\end{aligned}
$$

Thus, equation (2.6) holds true and $S_{X_{1}, X_{2}}\left(x_{1}, x_{2}\right)$ possesses the bivariate LMP. In fact, we have the following Lemma.

Lemma 3.6.3. Let $\left(T_{1}, T_{2}\right)$ and $T_{3}$ compose an EMO model with survival function given by (3.2). If $\left(T_{1}, T_{2}\right)$ exhibits bivariate LMP and $T_{3}$ is exponentially distributed, then $\left(X_{1}, X_{2}\right)=\left(\min \left(T_{1}, T_{3}\right), \min \left(T_{2}, T_{3}\right)\right)$ exhibits bivariate LMP.

Two related results are presented in next Lemmas. 
Lemma 3.6.4. Let $\left(T_{1}, T_{2}\right)$ and $T_{3}$ compose an EMO model with survival function given by (3.2). If $\left(X_{1}, X_{2}\right)=\left(\min \left(T_{1}, T_{3}\right), \min \left(T_{2}, T_{3}\right)\right)$ exhibits bivariate LMP and $T_{3}$ is exponentially distributed, then $\left(T_{1}, T_{2}\right)$ exhibits bivariate LMP.

Proof. From the general expression for survival function of EMO models (3.2) and the bivariate LMP of $\left(X_{1}, X_{2}\right)$ given by $(2.6)$ we have

$$
\begin{aligned}
S_{X_{1}, X_{2}}\left(x_{1}+t, x_{2}+t\right) & =S_{T_{1}, T_{2}}\left(x_{1}+t, x_{2}+t\right) S_{T_{3}}\left(\max \left(x_{1}+t, x_{2}+t\right)\right) \\
& =S_{T_{1}, T_{2}}\left(x_{1}, x_{2}\right) S_{T_{3}}\left(\max \left(x_{1}, x_{2}\right)\right) S_{T_{1}, T_{2}}(t, t) S_{T_{3}}(t),
\end{aligned}
$$

Since $T_{3}$ is exponentially distributed, $S_{T_{3}}\left(\max \left(x_{1}+t, x_{2}+t\right)\right)=S_{T_{3}}\left(\max \left(x_{1}, x_{2}\right)\right) S_{T_{3}}(t)$, and we conclude $S_{T_{1}, T_{2}}\left(x_{1}+t, x_{2}+t\right)=S_{T_{1}, T_{2}}\left(x_{1}, x_{2}\right) S_{T_{1}, T_{2}}(t, t)$.

Lemma 3.6.5. Let $\left(T_{1}, T_{2}\right)$ and $T_{3}$ compose an EMO model with survival function given by (3.2). If $\left(X_{1}, X_{2}\right)=\left(\min \left(T_{1}, T_{3}\right), \min \left(T_{2}, T_{3}\right)\right)$ and $\left(T_{1}, T_{2}\right)$ exhibit bivariate LMP then $T_{3}$ is exponentially distributed.

Proof. Follow similar steps as the proof of Lemma 3.6.4.

With the additional assumption of absolutely continuity of the distribution of $\left(T_{1}, T_{2}\right)$, in the next theorem we proof the unicity of the constructions presented in Lemmas 3.6.3, 3.6.4 and 3.6.5.

Theorem 3.6.6. Let $\left(T_{1}, T_{2}\right)$ and $T_{3}$ compose an EMO model with survival function given by (3.2). Suppose $\left(X_{1}, X_{2}\right)=\left(\min \left(T_{1}, T_{3}\right), \min \left(T_{2}, T_{3}\right)\right)$ exhibits bivariate LMP and the joint distribution of $\left(T_{1}, T_{2}\right)$ is absolutely continuous. Then $T_{3}$ is exponentially distributed and $\left(T_{1}, T_{2}\right)$ exhibits bivariate $L M P$.

Proof. Initially suppose $x_{1}>x_{2} \geq 0$ and consider the general expression for survival function of EMO models (3.5). Since $\left(X_{1}, X_{2}\right)$ exhibits bivariate LMP given by $(2.6)$,

$$
\begin{aligned}
& \exp \left\{-H_{T_{1}}\left(x_{1}+t\right)-H_{T_{3}}\left(x_{1}+t\right)-H_{T_{2}}\left(x_{2}+t\right)+H_{T_{1}, T_{2}}\left(x_{1}+t, x_{2}+t\right)\right. \\
& =\exp \left\{-H_{T_{1}}\left(x_{1}\right)-H_{T_{3}}\left(x_{1}\right)-H_{T_{2}}\left(x_{2}\right)+H_{T_{1}, T_{2}}\left(x_{1}, x_{2}\right)\right. \\
& \times \exp \left\{-H_{T_{1}}(t)-H_{T_{3}}(t)-H_{T_{2}}(t)+H_{T_{1}, T_{2}}(t, t) .\right.
\end{aligned}
$$

Taking logarithms in both sides and calculating the mixed partial derivatives, we obtain

$$
\frac{\partial^{2}}{\partial x_{1} \partial x_{2}} H_{T_{1}, T_{2}}\left(x_{1}+t, x_{2}+t\right)=\frac{\partial^{2}}{\partial x_{1} \partial x_{2}} H_{T_{1}, T_{2}}\left(x_{1}, x_{2}\right) \text {. }
$$

Integrating the previous expression and taking into account that $H_{T_{1}, T_{2}}\left(0, x_{2}\right)=H_{T_{1}, T_{2}}\left(x_{1}, 0\right)=0$ for all $x_{1}, x_{2} \geq 0$,

$$
\int_{0}^{x_{1}} \int_{0}^{x_{2}} \frac{\partial^{2}}{\partial u \partial v} H_{T_{1}, T_{2}}(u+t, v+t) \mathrm{d} v \mathrm{~d} u=\int_{0}^{x_{1}} \int_{0}^{x_{2}} \frac{\partial^{2}}{\partial u \partial v} H_{T_{1}, T_{2}}(u, v) \mathrm{d} v \mathrm{~d} u
$$

we obtain the relation

$$
H_{T_{1}, T_{2}}\left(x_{1}+t, x_{2}+t\right)-H_{T_{1}, T_{2}}\left(x_{1}+t, t\right)-H_{T_{1}, T_{2}}\left(t, x_{2}+t\right)+H_{T_{1}, T_{2}}(t, t)=H_{T_{1}, T_{2}}\left(x_{1}, x_{2}\right) .
$$


From the bivariate LMP of $\left(X_{1}, X_{2}\right)$ given by $(2.6)$ we have

$$
\frac{S_{X_{1}, X_{2}}\left(x_{1}, x_{2}\right)}{S_{X_{1}}\left(x_{1}\right) S_{X_{2}}\left(x_{2}\right)}=\frac{\frac{S_{X_{1}, X_{2}}\left(x_{1}+t, x_{2}+t\right)}{S_{X_{1}, X_{2}}(t, t)}}{\frac{S_{X_{1}, X_{2}}\left(x_{1}+t, t\right)}{S_{X_{1}, X_{2}}(t, t)} \frac{S_{X_{1}, X_{2}}\left(t, x_{2}+t\right)}{S_{X_{1}, X_{2}}(t, t)}} .
$$

Applying the exponential representation (3.3) to (3.24) and considering (3.23) we obtain the functional equation $H_{T_{3}}\left(x_{2}+t\right)=H_{T_{3}}\left(x_{2}\right)+H_{T_{3}}(t)$, for all $x_{2}, t \geq 0$. This is a Cauchy functional equation with only continuous solution given by $H_{T_{3}}\left(x_{2}\right)=\theta x_{2}$, see Sahoo and Kannappan (2011). Since $H_{T_{3}}\left(x_{2}\right)$ is nonnegative we have $\theta>0$. The same conclusion is obtained when $x_{2} \geq x_{1}$. Thus, $T_{3}$ is exponentially distributed with parameter $\theta$. Applying Lemma 3.6.4 we conclude the proof.

Remark 3.6.7. Relation (3.23) is an important characterizing functional equation and will be considered again in Lemma 7.2.2 as relation (7.1).

Corollary 3.6.8. Let $\left(T_{1}, T_{2}\right)$ and $T_{3}$ compose an EMO model with survival function given by (3.5) and the joint distribution of $\left(T_{1}, T_{2}\right)$ be absolutely continuous. Denote $\left(X_{1}, X_{2}\right)=\left(\min \left(T_{1}, T_{3}\right), \min \left(T_{2}, T_{3}\right)\right)$. Then $S_{X_{1}, X_{2}}\left(x_{1}, x_{2}\right)$ exhibits bivariate LMP if and only if $S_{T_{1}, T_{2}}\left(x_{1}, x_{2}\right)$ exhibits bivariate LMP and $T_{3}$ is exponentially distributed.

Example 3.6.9 (EMO model with bivariate LMP and $\left(T_{1}, T_{2}\right)$ absolutely continuous). Consider the joint survival function for $\left(T_{1}, T_{2}\right)$

$$
S_{T_{1}, T_{2}}\left(x_{1}, x_{2}\right)=\frac{\lambda}{\lambda_{1}+\lambda_{2}} \exp \left\{-\lambda_{1} x_{1}-\lambda_{2} x_{2}-\lambda_{12} \max \left(x_{1}, x_{2}\right)\right\}-\frac{\lambda_{12}}{\lambda_{1}+\lambda_{2}} \exp \left\{-\lambda \max \left(x_{1}, x_{2}\right)\right\},
$$

where the parameters $\lambda_{1}, \lambda_{2}$ and $\lambda_{12}$ are positive, $\lambda=\lambda_{1}+\lambda_{2}+\lambda_{12}$ and $x_{1}, x_{2}>0$, i.e., $\left(T_{1}, T_{2}\right)$ follows the Block and Basu (1974) bivariate exponential distribution, which is an example of an absolutely continuous distribution with bivariate LMP. Let $T_{3}$ follow an exponential distribution with parameter $\theta>0$, so that $S_{T_{3}}(x)=\exp \{-\theta x\}$. Therefore

$$
S_{X_{1}, X_{2}}=S_{T_{1}, T_{2}}\left(x_{1}, x_{2}\right) S_{T_{3}}\left(\max \left(x_{1}, x_{2}\right)\right)
$$

compose an EMO model with the bivariate LMP.

Remark 3.6.10 (Reliability property of marginals). From the exponential representation for EMO models (3.5), let $x_{3-i}=0$ and calculate the failure rate functions of $X_{i}$ to obtain $r_{X_{i}}\left(x_{i}\right)=$ $r_{T_{i}}\left(x_{i}\right)+r_{T_{3}}\left(x_{i}\right), i=1,2$. Fixing for $T_{3}$ an exponential distribution with failure rate $r_{T_{3}}(x)=\lambda_{3}$, $\lambda_{3}>0$, the increasing (decreasing) behaviour of $r_{X_{i}}(x), i=1,2$, is solely determined by the increasing (decreasing) behaviour of $r_{T_{i}}(x), i=1,2$ (in Example 3.6.9, $X_{1}$ and $X_{2}$ are both IFR, see Block and Basu (1974)). Therefore, it is possible to obtain EMO models with bivariate LMP with any combination of increasing or decreasing failure rate property of the marginals $X_{1}$ and $X_{2}$, see Remark 3 in Kulkarni (2006) for details.

In next theorem we show that all EMO models which possess the bivariate LMP have a singular component, i.e., are not absolutely continuous.

Theorem 3.6.11. Let $\left(T_{1}, T_{2}\right)$ and $T_{3}$ compose an EMO model with survival function given by (3.2) and denote $\left(X_{1}, X_{2}\right)=\left(\min \left(T_{1}, T_{3}\right), \min \left(T_{2}, T_{3}\right)\right)$. If $\left(X_{1}, X_{2}\right)$ possess the bivariate LMP then $S_{X_{1}, X_{2}}\left(x_{1}, x_{2}\right)$ is not absolutely continuous. 
Proof. Suppose $S_{X_{1}, X_{2}}\left(x_{1}, x_{2}\right)$ possess the bivariate LMP and is absolutely continuous. From the bivariate LMP we have $X=\min \left(X_{1}, X_{2}\right)$ is exponentially distributed. But from the absolutely continuity of $S_{X_{1}, X_{2}}\left(x_{1}, x_{2}\right)$, Theorem 3.5.3 holds true and this bivariate survival function is given by the general expression (3.22), meaning that the support of the distribution of $X$ is limited from above by $\theta$. Thus, $X$ is not exponentially distributed, which is a contradiction. Therefore we conclude $S_{X_{1}, X_{2}}\left(x_{1}, x_{2}\right)$ is not absolutely continuous.

\subsubsection{Weak bivariate increasing failure rate}

Under bivariate LMP property, the ratio

$$
D\left(x_{1}, x_{2}, t\right)=\frac{S_{X_{1}, X_{2}}\left(x_{1}+t, x_{2}+t\right)}{S_{X_{1}, X_{2}}\left(x_{1}, x_{2}\right)}
$$

only depends on $t$ and is non-increasing, see the defining bivariate LMP relation (2.6). Considering EMO models, we now investigate the increasing/decreasing nature of the ratio (3.25) in $x_{1}, x_{2} \geq 0$ for all $t \geq 0$.

Finkelstein and Esaulova (2005) defined the concept of weak bivariate increasing failure rate IFR (decreasing failure rate - DFR) considering a particular version of (3.25), when $x_{1}=x_{2}=x \geq 0$.

Definition 3.6.12 (Finkelstein and Esaulova (2005)). The joint distribution of a bivariate random vector $\left(X_{1}, X_{2}\right)$ is weak increasing failure rate - IFR (decreasing failure rate - DFR) if

$$
D(x, x, t)=\frac{S_{X_{1}, X_{2}}(x+t, x+t)}{S_{X_{1}, X_{2}}(x, x)}
$$

is decreasing (increasing) in $x \geq 0$ for all $t \geq 0$.

This definition tells us that the residual lifetime of a series system of two components with the same initial ages stochastically decreases (increases) in $x$ for all $t \geq 0$. Following Finkelstein and Esaulova (2005), the terms increasing and decreasing are not strict, i.e., increasing means non-decreasing as well as decreasing means non-increasing.

Finkelstein and Esaulova (2005) established conditions to an absolutely continuous bivariate survival function be weak IFR (DFR) as well as proposed a methodology to construct bivariate distributions satisfying the weak IFR (DFR) aging property from bivariate distributions with the same property. If we consider the absolutely continuous version of EMO model given by Theorem 3.5.3, log-concavity properties (e.g. Barlow and Proschan (1981)) imply a general condition for the weak IFR aging property:

$$
\frac{d^{2}}{d x^{2}} \ln \left[S_{X_{1}, X_{2}}(x, x)\right] \leq 0 .
$$

From the general expression for survival function of absolutely continuous EMO models (3.22), when $x_{1}=x_{2}=x \geq 0, S_{X_{1}, X_{2}}(x, x)=S_{T_{1}, T_{2}}(x, x), x \in(0, \theta), \theta$ defined in Theorem 3.5.3. In fact, to investigate the weak aging property of the absolutely continuous EMO models we only have to analyse the IFR (DFR) aging property of $T=\min \left(T_{1}, T_{2}\right)$. Therefore, we need to investigate bivariate distributions for $\left(T_{1}, T_{2}\right)$ that satisfy such property.

From Theorem 3.5.3, the support of $T=\min \left(T_{1}, T_{2}\right)$ is limited from above by $\theta$. Since $\int_{0}^{\theta} r_{T}(u) \mathrm{d} u=$ $\infty$, where $r_{T}(x)$ is the failure rate function of $T, r_{T}(x)$ has to be unbounded from above and, con- 
sequently, is not decreasing in $x$ for all $x \in[0, \theta)$. This rules out the possibility of an absolutely continuous EMO models with the weak DFR property. Thus, we have proved the following Lemma.

Lemma 3.6.13. Let the bivariate random vector $\left(X_{1}, X_{2}\right)$ follow the absolutely continuous EMO model from Theorem 3.5.3. Then $S_{X_{1}, X_{2}}\left(x_{1}, x_{2}\right)$ does not exhibit the weak DFR property.

In the next example, condition (3.27) is applied to an absolutely continuous EMO model to investigate its weak IFR aging property.

Example 3.6.14 (Absolutely continuous EMO model and weak IFR). Consider the absolutely continuous EMO model presented in Example 3.5.4. To analyse its weak aging property, it is necessary to take into account only the behaviour of

$$
S_{T_{1}, T_{2}}\left(x_{1}, x_{2}\right)=\left(\exp \left\{-\frac{x_{1}}{c}\right\}+\exp \left\{-\frac{x_{2}}{c}\right\}-1\right)^{c},
$$

since our interest is in the performance of $S_{X_{1}, X_{2}}\left(x_{1}, x_{2}\right)$ when $x_{1}=x_{2}=x \geq 0$, and $S_{X_{1}, X_{2}}(x, x)=$ 0 for $x>c \ln 2$. Therefore,

$$
\frac{d^{2}}{d x^{2}} \ln \left[\left(2 \exp \left\{-\frac{x}{c}\right\}-1\right)^{c}\right]=-\frac{2 \exp \left\{-\frac{x}{c}\right\}}{c\left(2 \exp \left\{-\frac{x}{c}\right\}-1\right)^{2}} \leq 0,
$$

for all $x \in(0, c \ln 2)$ and the distribution of $\left(X_{1}, X_{2}\right)$ is weak IFR. Once the marginal distributions of $T_{1}$ and $T_{2}$ are unit exponentials, from Theorems 2 and 3 in Finkelstein and Esaulova (2005), we can conclude that the weak IFR aging property still holds true with arbitrary identical IFR marginals for $\left(T_{1}, T_{2}\right)$ as well as with arbitrary IFR marginals with proportional failure rates, provided we maintain the same copula for $\left(T_{1}, T_{2}\right)$. Notice that when the distributions of $T_{1}$ and $T_{2}$ are changed, the support of the distribution of the absolutely continuous random variable $T_{3}$ may have to be adjusted in order to avoid singularities in the EMO model, according with Theorem 3.5.3.

To investigate the weak IFR property of the absolutely continuous EMO model, notice the restriction to be imposed to the absolutely continuous random variable $T_{3}$ is uniquely related with its support, necessary to the EMO model be absolutely continuous, and we only have to analyse the behaviour of the failure rate of the random variable $T=\min \left(T_{1}, T_{2}\right)$. From the exponential representation for bivariate survival functions (3.3), condition (3.27) is equivalent to

$$
r_{T_{1}}(x)+r_{T_{2}}(x)-\frac{d}{d x} H_{T_{1}, T_{2}}(x, x)
$$

be increasing in $x, x \in(0, \theta)$, where $\theta$ is defined in Theorem 3.5.3.

Notice that from the definition of conditional hazard rates and from the exponential representation (3.3), the failure rates of $T_{i}, i=1,2$ can be expressed as

$$
r_{T_{i}}\left(x_{i}\right)=r_{i}\left(x_{1}, x_{2}\right)+\frac{\partial}{\partial x_{i}} H_{T_{1}, T_{2}}\left(x_{1}, x_{2}\right), i=1,2 .
$$

Moreover, whenever $H_{T_{1}, T_{2}}\left(x_{1}, x_{2}\right)$ is differentiable, the derivative $\frac{d}{d x} H_{T_{1}, T_{2}}(x, x)$ can be written in terms of the gradient of $H_{T_{1}, T_{2}}\left(x_{1}, x_{2}\right)$ as

$$
\frac{d}{d x} H_{T_{1}, T_{2}}(x, x)=\left.\frac{\partial}{\partial x_{1}} H_{T_{1}, T_{2}}\left(x_{1}, x_{2}\right)\right|_{x_{1}=x_{2}=x}+\left.\frac{\partial}{\partial x_{2}} H_{T_{1}, T_{2}}\left(x_{1}, x_{2}\right)\right|_{x_{1}=x_{2}=x},
$$


see Remark 5.2.4. Therefore, we have proved the following Lemma.

Lemma 3.6.15. Let the bivariate random vector $\left(X_{1}, X_{2}\right)$ follow the absolutely continuous EMO model from Theorem 3.5.3 and $H_{T_{1}, T_{2}}\left(x_{1}, x_{2}\right)$ be a differentiable dependence function. Then $\left(X_{1}, X_{2}\right)$ is weak IFR if and only if $r_{1}(x, x)+r_{2}(x, x)$ is increasing in $x$.

The next example illustrates an application of Lemma 3.6.15.

Example 3.6.16. Consider the absolutely continuous EMO model presented in Example 3.6.14. The conditional hazard rates are given by $r_{i}\left(x_{1}, x_{2}\right)=\frac{\exp \left\{-\frac{x_{i}}{c}\right\}}{\exp \left\{-\frac{x_{1}}{c}\right\}+\exp \left\{-\frac{x_{2}}{c}\right\}-1}, i=1,2$. Therefore, $r_{1}(x, x)+r_{2}(x, x)=\frac{2 \exp \left\{-\frac{x}{c}\right\}}{2 \exp \left\{-\frac{x}{c}\right\}-1}$ and

$$
\frac{d}{d x}\left(\frac{2 \exp \left\{-\frac{x}{c}\right\}}{2 \exp \left\{-\frac{x}{c}\right\}-1}\right)=\frac{2 \exp \left\{-\frac{x}{c}\right\}}{c\left(2 \exp \left\{-\frac{x}{c}\right\}-1\right)^{2}} \geq 0,
$$

for all $x \in(0, c \ln 2)$. From Lemma 3.6.15 we conclude that the distribution of $\left(X_{1}, X_{2}\right)$ is weak IFR.

There exists a connection between bivariate distributions with the property that the minimum is increasing failure rate and log-concave functions, whose definition can be found in $\mathrm{Hu}$ and $\mathrm{Li}$ (2007).

Definition 3.6.17 (Hu and $\mathrm{Li}$ (2007)). A non-negative function $\gamma: \mathbf{R}^{\mathbf{n}} \rightarrow \mathbf{R}$ is said to be $\log$ concave if, for all $\mathbf{x}, \mathbf{y} \in \mathbf{R}^{\mathbf{n}}$ and for all $\alpha \in(0,1)$ we have

$$
\gamma(\alpha \mathbf{x}+(1-\alpha) \mathbf{y}) \geq[\gamma(\mathbf{x})]^{\alpha}[\gamma(\mathbf{y})]^{1-\alpha}
$$

This definition is equivalent to $\ln \gamma(\mathbf{x})$ be concave. As noted in An (1998), if the function $\gamma(\mathbf{x})$ is log-concave, then the set $\mathbf{\Upsilon}=\left\{\mathbf{x} \in \mathbf{R}^{\mathbf{n}}: \gamma(\mathbf{x})>\mathbf{0}\right\}$ is a convex subset of $\mathbf{R}^{\mathbf{n}}$.

Denoting by $\Gamma(\mathbf{x})=-\ln (\gamma(\mathbf{x}))$, Definition 3.6.17 is equivalent to the Hessian matrix associated with $\Gamma(\mathbf{x})$ be nonnegative definite, provided the elements of the Hessian matrix exist and are continuous in $\mathbf{\Upsilon}$, see Proposition 3.67 in Ghorpade and Limaye (2010).

Interesting properties of log-concave and log-convex functions in the univariate case can be found in Bagnoli and Bergstrom (2005) and Al-Zahrani and Stoyanov (2008). An (1998), besides the univariate case, also presents some results to the multivariate case. Walther (2009) presents inferential and modeling aspects of log-concave distributions.

$\mathrm{Hu}$ and $\mathrm{Li}$ (2007) investigated increasing failure rate properties of the minimum of multivariate distributions with log-concave densities, and they proved the following theorem.

Theorem 3.6.18 (Hu and $\mathrm{Li}(2007))$. Let $\left(X_{1}, \ldots, X_{n}\right)$ be a random vector with a log-concave density function. Then $T=\min \left(X_{1}, \ldots, X_{n}\right)$ is IFR.

Remark 3.6.19 (Truncation of log-concave density functions to convex sets). As observed in $H u$ and $L i$ (2007), a multivariate random vector with log-concave density function truncated to a convex set possesses a log-concave density function. In the bivariate case, the corresponding survival function can be used as $S_{T_{1}, T_{2}}\left(x_{1}, x_{2}\right)$ in Example 3.6.14, provided the distribution of $T_{3}$ is chosen such that the conditions of Theorem 3.5.3 hold true. 
Remark 3.6.20 (Multivariate log-concave densities). Examples of multivariate log-concave densities can be found in $\mathrm{Hu}$ and $\mathrm{Li}$ (2007), such as multivariate normal and multivariate Pearson type II distributions, and in Gupta and Balakrishnan (2012), such as multivariate skew-normal models of Azzalini (1985) and of Azzalini and Dalla Valle (1996).

\subsubsection{Bivariate increasing hazard rate}

Several generalizations of the univariate increasing (decreasing) failure rate definition to the multivariate setup can be found in the literature. Among others, we can mention Harris (1970), Basu (1971) and Brindley and Thompson (1972), see Definition 6.3.2 in Section 6.3. Johnson and Kotz (1975) present the definition of Multivariate Increasing Hazard Rate - MIHR (Decreasing Hazard Rate - MDFR), which, in the bivariate case is

Definition 3.6.21 (Johnson and Kotz (1975)). An absolutely continuous random vector $\left(X_{1}, X_{2}\right)$ with survival function $S_{X_{1}, X_{2}}\left(x_{1}, x_{2}\right)$ is Bivariate Increasing Hazard Rate - BIHR (Decreasing Hazard Rate - BDHR) if

$$
\frac{\partial}{\partial x_{i}}\left(-\ln S_{X_{1}, X_{2}}\left(x_{1}, x_{2}\right)\right)
$$

is an increasing (decreasing) function of $x_{i}$ for all values of $\left(x_{1}, x_{2}\right), i=1,2$. .

Note that if $X_{1}$ and $X_{2}$ are independent, their joint distribution is IHR (DHR) if and only if the marginal distributions of $X_{1}$ and $X_{2}$ are IFR (DFR). In other words, Definition 3.6.21 includes the IFR (DFR) notion assumed by Barlow and Proschan (1981).

This property has been investigated in Gupta and Gupta (1997), Ma (2000), Gupta and Gupta (2001), Horrance (2005), Hu and Li (2007) and Gupta and Balakrishnan (2012), among others, for some multivariate distributions.

The following theorem, whose multivariate version is proved in Ma (2000), relates the logconcavity of a survival function $S_{X_{1}, X_{2}}\left(x_{1}, x_{2}\right)$ to the monotonicity of its conditional hazard rates $r_{i}\left(x_{1}, x_{2}\right)$ as a function of $x_{i}, i=1,2$.

Theorem 3.6.22 (Ma (2000)). Let $\left(X_{1}, X_{2}\right)$ be an absolutely continuous random vector with survival function $S_{X_{1}, X_{2}}\left(x_{1}, x_{2}\right)$. If $S_{X_{1}, X_{2}}\left(x_{1}, x_{2}\right)$ is log-concave, then the random vector $\left(X_{1}, X_{2}\right)$ is bivariate IHR.

The next theorem, whose multivariate version proof can be found in An (1998), shows that bivariate log-concave densities generates bivariate log-concave survival distributions, as well as their univariate marginal densities are also log-concave.

Theorem 3.6.23 (An (1998)). If $f_{X_{1}, X_{2}}\left(x_{1}, x_{2}\right)$ is log-concave, then:

1. $S_{X_{1}, X_{2}}\left(x_{1}, x_{2}\right)$ is log-concave;

2. $f_{X_{i}}\left(x_{i}\right), i=1,2$ are log-concave.

Theorem 3.6.24 shows how an absolutely continuous EMO model satisfying the bivariate IHR property can be obtained.

Theorem 3.6.24. Let the bivariate random vector $\left(X_{1}, X_{2}\right)$ follow the absolutely continuous EMO model from Theorem 3.5.3. If $\left(T_{1}, T_{2}\right)$ is bivariate IHR and $T_{3}$ is IFR, then $\left(X_{1}, X_{2}\right)$ is bivariate IHR. 
Proof. Consider the general expression for survival function of absolutely continuous EMO models (3.22),

$$
S_{X_{1}, X_{2}}\left(x_{1}, x_{2}\right)= \begin{cases}S_{T_{1}, T_{2}}\left(x_{1}, x_{2}\right), & \text { if } 0 \leq x_{1}, x_{2}<\gamma \\ S_{T_{1}, T_{2}}\left(x_{1}, x_{2}\right) S_{T_{3}}\left(x_{1}\right), & \text { if } x_{1} \geq \min \left(x_{2}, \gamma\right) \\ S_{T_{1}, T_{2}}\left(x_{1}, x_{2}\right) S_{T_{3}}\left(x_{2}\right), & \text { if } x_{2} \geq \min \left(x_{1}, \gamma\right) .\end{cases}
$$

For $i=1,2$ we have

$$
\frac{\partial}{\partial x_{i}}\left(-\ln \left(S_{X_{1}, X_{2}}\left(x_{1}, x_{2}\right)\right)\right)= \begin{cases}\frac{\partial}{\partial x_{i}}\left(-\ln \left(S_{T_{1}, T_{2}}\left(x_{1}, x_{2}\right)\right)\right), & \text { if } 0 \leq x_{1}, x_{2}<\gamma \\ \frac{\partial}{\partial x_{i}}\left(-\ln \left(S_{T_{1}, T_{2}}\left(x_{1}, x_{2}\right)\right)\right)+\frac{d}{d x_{i}}\left(-\ln \left(S_{T_{3}}\left(x_{1}\right)\right)\right), & \text { if } x_{1} \geq \min \left(x_{2}, \gamma\right), \\ \frac{\partial}{\partial x_{i}}\left(-\ln \left(S_{T_{1}, T_{2}}\left(x_{1}, x_{2}\right)\right)\right)+\frac{d}{d x_{i}}\left(-\ln \left(S_{T_{3}}\left(x_{2}\right)\right)\right), & \text { if } x_{2} \geq \min \left(x_{1}, \gamma\right) .\end{cases}
$$

Thus, $\frac{d}{d x_{i}}\left(-\ln \left(S_{T_{3}}\left(x_{1}\right)\right)\right)$ and $\frac{d}{d x_{i}}\left(-\ln \left(S_{T_{3}}\left(x_{2}\right)\right)\right)$ are equal to 0 or to the increasing failure rate function of $T_{3}$, depending on the value of $i, i=1$, Since $\left(T_{1}, T_{2}\right)$ is bivariate IHR, then $\frac{\partial}{\partial x_{i}}\left(-\ln \left(S_{X_{1}, X_{2}}\left(x_{1}, x_{2}\right)\right)\right)$ is increasing in $x_{i}$ for all values of $\left(x_{1}, x_{2}\right)$, see Definition 3.6.21, what concludes the proof.

The next example illustrates an application of Theorem 3.6.24.

Example 3.6.25 (Absolutely continuous EMO model and bivariate IHR). Consider the survival function already considered in Example 3.4.4,

$$
S_{T_{1}, T_{2}}\left(x_{1}, x_{2}\right)=\left\{\left(1-p_{1} x_{1}-p_{2} x_{2}\right)\right\}^{c}
$$

where $0<x_{1}<\frac{1}{p_{1}}, 0<x_{2}<\frac{1-p_{1} x_{1}}{p_{2}}$ and $p_{1}, p_{2}>0, c>1$, i.e., the bivariate case of the multivariate rescaled Dirichlet distribution presented in Ma (1996), see also equation (9.32) in Balakrishnan and Lai (2009). Suppose $T_{3}$ follows a Weibull distribution with location parameter $\frac{1}{p_{1}+p_{2}}$,

$$
S_{T_{3}}(x)=\exp \left\{-\left(x-\frac{1}{p_{1}+p_{2}}\right)^{b}\right\},
$$

where $x \geq \frac{1}{p_{1}+p_{2}}$ and $b>1$. Notice that Theorem 3.5.3 holds true, i.e., $\left(X_{1}, X_{2}\right)=\left(\min \left(T_{1}, T_{3}\right), \min \left(T_{2}, T_{3}\right)\right)$ compose an absolutely continuous EMO model. The survival function $S_{T_{1}, T_{2}}\left(x_{1}, x_{2}\right)$ is log-concave, since calculating the elements of the Hessian matrix $\mathcal{H}$ associated with the function $-\ln S_{T_{1}, T_{2}}\left(x_{1}, x_{2}\right)$ we have

$$
\mathcal{H}=\frac{c}{\left(1-p_{1} x_{1}-p_{2} x_{2}\right)^{2}}\left[\begin{array}{cc}
p_{1}^{2} & p_{1} p_{2} \\
p_{1} p_{2} & p_{2}^{2}
\end{array}\right] .
$$

Once $p_{1}^{2} \geq 0, p_{2}^{2} \geq 0$ and $p_{1}^{2} p_{1}^{2}-\left(p_{1} p_{2}\right)\left(p_{1} p_{2}\right) \geq 0$, we have $\mathcal{H}$ nonnegative definite and $S_{T_{1}, T_{2}}\left(x_{1}, x_{2}\right)$ is log-concave. From Theorem 3.6.22, $\left(T_{1}, T_{2}\right)$ is bivariate IFR. Because $T_{3}$ is IFR, from Theorem 
3.6.24, the absolutely continuous EMO model given by

$$
\begin{aligned}
& S_{X_{1}, X_{2}}\left(x_{1}, x_{2}\right)= \\
& = \begin{cases}\left\{\left(1-p_{1} x_{1}-p_{2} x_{2}\right)\right\}^{c}, & \text { if }\left(x_{1}, x_{2}\right) \in \mathbf{D}, 0 \leq x_{1}, x_{2}<\frac{1}{p_{1}+p_{2}} ; \\
\left\{\left(1-p_{1} x_{1}-p_{2} x_{2}\right)\right\}^{c} \exp \left\{-\left(x_{1}-\frac{1}{p_{1}+p_{2}}\right)^{b}\right\}, & \text { if }\left(x_{1}, x_{2}\right) \in \mathbf{D}, x_{1} \geq \min \left(x_{2}, \frac{1}{p_{1}+p_{2}}\right) ; \\
\left\{\left(1-p_{1} x_{1}-p_{2} x_{2}\right)\right\}^{c} \exp \left\{-\left(x_{2}-\frac{1}{p_{1}+p_{2}}\right)^{b}\right\}, & \text { if }\left(x_{1}, x_{2}\right) \in \mathbf{D}, x_{2} \geq \min \left(x_{1}, \frac{1}{p_{1}+p_{2}}\right),\end{cases}
\end{aligned}
$$

where $\mathbf{D}=\left\{\left(x_{1}, x_{2}\right): x_{1} \geq 0, x_{2} \geq 0\right.$ and $\left.1-p_{1} x_{1}-p_{2} x_{2} \geq 0\right\}$ is bivariate IHR.

Remark 3.6.26. Bagnoli and Bergstrom (2005) list several nonnegative univariate distributions with log-concave densities which generates log-concave survival distributions. Such distributions can be chosen as the distribution for $T_{3}$ in Example 3.6.25, provided a location parameter is introduced so that Theorem 3.5.3 holds true. From Theorem 3.6.23, log-concave bivariate survival functions can be obtained from log-concave bivariate density functions. Remarks 3.6.19 and 3.6.20 remain useful when analysing the bivariate IHR property.

\subsection{Extreme value EMO models}

In this section we investigate properties of extreme value EMO models. In the following two subsections we give notations and review the necessary facts to make the exposition self-containing. In the last subsection we obtain extreme value EMO models, their corresponding survival copulas and Pickands measure.

\subsubsection{Univariate and bivariate extreme value distributions}

The univariate theory of extreme value has been extensively analysed, see Galambos (1978), Beirlant et al. (2005) and the references therein for a complete exposition. Start with a sequence of independent and identically distributed random variables $X_{i}, i=1, \ldots, n$, with distribution function $F(x)$, and define $M_{n}=\max \left(X_{1}, \ldots X_{n}\right)$. Suppose that there exists a sequence of normalizing constants $a_{n}>0$ and $b_{n}$ such that when $n \rightarrow \infty$ we have the convergence in distribution,

$$
\mathbb{P}\left(\frac{M_{n}-b_{n}}{a_{n}} \leq z\right)=F^{n}\left(a_{n} z+b_{n}\right) \rightarrow G(z)
$$

where $G(z)$ is a nondegenerate distribution function.

In this case, $G(z)$ must be a member of the class of the Generalized Extreme Value (GEV) distributions, i.e., location-scale families of distributions based on Weibull, Fréchet and Gumbel distributions, see Theorem 2.4.1 of Galambos (1978), and $F(x)$ is in the domain of attraction of $G(z)$. The sequence of normalizing constants need not be unique, and the effect of different and appropriate choices for such constants is a change in the location and scale parameters of the limiting distribution $G(z)$.

Considering that $W_{n}=\min \left(X_{1}, \ldots X_{n}\right)=-\max \left(-X_{1}, \ldots-X_{n}\right)$, similar results hold for $W_{n}$, see Theorem 2.4.2 of Galambos (1978). Necessary and sufficient conditions for the weak convergence 
of the distributions of $M_{n}$ and $W_{n}$ to a member of the class of the GEV distributions can be found in Theorems 2.4.3 and 2.4.4 of Galambos (1978).

The extension of the analysis of extreme value distributions for the bivariate case is not immediate, since there is not a unique way to order bivariate observations, see Barnett (1976). Here we will consider the most widely used approach, the componentwise ordering.

Consider $n$ independent and identically distributed bivariate random vectors $\mathbf{X}_{\mathbf{i}}=\left(X_{1, i}, X_{2, i}\right)$, $i=1, \ldots, n$, and define $\mathbf{M}_{\mathbf{n}}=\left(M_{1, n}, M_{2, n}\right)$ such that

$$
M_{j, n}=\max \left\{X_{j, i}, i=1, \ldots n\right\}, \quad \mathrm{j}=1,2 .
$$

Note that since $M_{1, n}$ and $M_{2, n}$ are componentwise maxima, $\mathbf{M}_{\mathbf{n}}$ need not to be an observation, i.e., need not belong to the set $\left\{\mathbf{X}_{\mathbf{i}}, i=1, \ldots n\right\}$.

Analogously to the univariate theory, if there exist sequences of normalizing constants $a_{j, n}>0$ and $b_{j, n}, j=1,2$, such that when $n \rightarrow \infty$ we have the convergence in distribution,

$$
\mathbb{P}\left(\frac{M_{1, n}-b_{1, n}}{a_{1, n}} \leq x_{1}, \frac{M_{2, n}-b_{2, n}}{a_{2, n}} \leq x_{2}\right)=F_{X_{1}, X_{2}}^{n}\left(a_{1, n} x_{1}+b_{1, n}, a_{2, n} x_{2}+b_{2, n}\right) \rightarrow G\left(x_{1}, x_{2}\right),
$$

where $G\left(x_{1}, x_{2}\right)$ is a proper distribution function, nondegenerate in each margin, then $G\left(x_{1}, x_{2}\right)$ is a bivariate extreme value distribution and $F_{X_{1}, X_{2}}\left(x_{1}, x_{2}\right)$ is in its domain of attraction.

Whenever the limiting distribution $G\left(x_{1}, x_{2}\right)$ exists, it is continuous, see Theorem 5.2 .2 of Galambos (1978). Its analysis can be split in two parts: the marginal distributions and the dependence structure.

Concerning the marginal distributions, we have the convergence in distribution of the marginal distributions of $F_{X_{1}, X_{2}}^{n}\left(x_{1}, x_{2}\right)$ to the marginal distributions of $G\left(x_{1}, x_{2}\right)$. Moreover, the marginal distributions of $G\left(x_{1}, x_{2}\right)$ belong to the class of the GEV distributions, see Lemma 5.2.1 and Theorem 5.2.2 in Galambos (1978).

Since the class of GEV distributions is composed by continuous distributions, by Sklar's Theorem, see Theorem 2.2.3 in Nelsen (2006), the bivariate copula $C(u, v)$ associated with $G\left(x_{1}, x_{2}\right)$ is unique and can be used to investigate the dependence structure of bivariate extreme value distributions. Therefore, we have

$$
G\left(x_{1}, x_{2}\right)=C\left(V_{1}\left(x_{1}\right), V_{2}\left(x_{2}\right)\right)
$$

where $V_{i}\left(x_{i}\right), i=1,2$, belong to the class of GEV distributions.

The copula $C(u, v)$ in $(3.28)$ is such that

$$
[C(u, v)]^{q}=C\left(u^{q}, v^{q}\right),
$$

where $q>0$, see Theorem 5.2.1 and Lemma 5.4.1 of Galambos (1978). Copulas that satisfy (3.29) are denominated extreme value copulas. Examples of extreme value copulas are the independence, comonotonicity (see Remark 3.2.11), Gumbel-Hougaard (see relation (3.42) in Example 3.7.18) and Marshall-Olkin (see relation (2.15)) survival copulas, among others.

The characterization of bivariate extreme value distributions can be done by means of Theorem 5.2.4 of Galambos (1978): $G\left(x_{1}, x_{2}\right)$ is a bivariate extreme value distribution if and only if its marginal distributions are members of the class of GEV distributions and relation (3.29) is verified. 


\subsubsection{Pickands representation for bivariate extreme value copulas}

Other characterizations for bivariate extreme value copulas are possible, such as the Pickands representation, see Pickands (1981). Following McNeil et al. (2005), such copulas can be completely characterized as

$$
C(u, v)=\exp \left\{(\ln u v) \mathcal{A}\left(\frac{\ln u}{\ln u v}\right)\right\}
$$

where $u, v \in(0,1), \mathcal{A}(w)=\int_{0}^{1} \max ((1-x) w, x(1-w)) d \mathbb{H}(x)$, for a positive finite measure $\mathbb{H}$ on $[0,1]$, denominated Pickands measure.

The function $\mathcal{A}(w):[0,1] \rightarrow\left[\frac{1}{2}, 1\right]$, named Pickands dependence function, besides being convex satisfies

$$
\max (w, 1-w) \leq \mathcal{A}(w) \leq 1
$$

The Pickands dependence function can be extracted from the corresponding copula as

$$
\mathcal{A}(w)=-\ln C(\exp \{-w\}, \exp \{-(1-w)\}) \quad w \in[0,1],
$$

see McNeil et al. (2005) for details, and uniquely corresponds to the measure $\mathbb{H}$,

$$
\mathbb{H}([0, w])= \begin{cases}1+\frac{d}{d w} \mathcal{A}(w), & \text { if } w \in[0,1) \\ 2, & \text { if } w=1\end{cases}
$$

where $\frac{d}{d w} \mathcal{A}(w)$ is the right-hand derivative of $\mathcal{A}(w)$, see Theorem 3.1 in Pickands (1981) and Section 8.2.5 in Beirlant et al. (2005). Moreover, the point masses of $\mathbb{H}$ at 0 and 1 are given by

$$
\mathbb{H}(\{0\})=1+\frac{d}{d w} \mathcal{A}(0) \quad \text { and } \quad \mathbb{H}(\{1\})=1-\frac{d}{d w} \mathcal{A}(1),
$$

where $\frac{d}{d w} \mathcal{A}(1)=\sup _{0 \leq w<1} \frac{d}{d w} \mathcal{A}(w)$.

Two important examples of Pickands dependence functions are:

- the lower bound $\mathcal{A}(w)=\max (w, 1-w), w \in[0,1]$, that corresponds to the comonotonicity copula. The corresponding Pickands measure puts mass 2 at $w=\frac{1}{2}$;

- the upper bound $\mathcal{A}(w)=1, w \in[0,1]$, that corresponds to the independence copula. The corresponding Pickands measure assigns mass 1 to both $w=0$ and $w=1$.

\subsubsection{Construction of extreme value EMO models}

When bivariate extreme value copulas take on univariate GEV distributions one obtains bivariate extreme value distributions for maxima, but when the copulas take on GEV survival margins for minima, bivariate extreme value survival functions for minima are obtained, see page 176 in Joe (1997). In particular, considering relation (3.29) and exponential survival marginals, we conclude that if $S_{X_{1}, X_{2}}\left(x_{1}, x_{2}\right)$ is an extreme value survival function with exponential marginals, then for $q>0$

$$
S_{X_{1}, X_{2}}\left(q x_{1}, q x_{2}\right)=\left[S_{X_{1}, X_{2}}\left(x_{1}, x_{2}\right)\right]^{q} .
$$

Remark 3.7.1 (Bivariate extreme value distributions with Fréchet marginals). Considering relation (3.29), if $F_{X_{1}, X_{2}}\left(x_{1}, x_{2}\right)$ is an extreme value distribution function with Fréchet marginals, where the 
shape parameter $\alpha=1$ and scale parameter $s_{i}>0$, i.e., $F_{X_{i}}\left(x_{i}\right)=\exp \left\{-\left(\frac{x_{i}}{s_{i}}\right)^{-1}\right\}, i=1,2$, then for $q>0$ we obtain the expression $F_{X_{1}, X_{2}}^{q}\left(q x_{1}, q x_{2}\right)=F_{X_{1}, X_{2}}\left(x_{1}, x_{2}\right)$.

It is possible to obtain a family of extreme value survival copulas derived from extreme value EMO models. Notice that the survival function of the EMO model (3.5) can be equivalently presented as

$$
S_{X_{1}, X_{2}}\left(x_{1}, x_{2}\right)=\left\{S_{T_{1}, T_{2}}\left(x_{1}, x_{2}\right)\right\}\left\{\min \left[S_{T_{3}}\left(x_{1}\right), S_{T_{3}}\left(x_{2}\right)\right]\right\} .
$$

Remark 3.7.2. Equation (3.34) can be interpreted as a product of two bivariate survival functions, where the last one refers to a bivariate random vector with comonotonic components sharing the same marginal distribution as $T_{3}$, see related Remark 3.2.11.

Since the margins of any multivariate extreme value distribution must be a member of the class of univariate GEV distributions, for simplicity we can choose exponential margins for $X_{1}$ and $X_{2}$ in the alternative representation of EMO models (3.34). That has no influence on the copula to be obtained, for strict monotone transformations can be used to move from one member to the other in the class of GEV distributions, see Galambos (1978), Tawn (1988) and Beirlant et al. (2005) for a discussion on that.

Analysing the survival function of EMO models given by (3.2), in order to obtain $X_{i}$ exponentially distributed with parameter $\lambda_{X_{i}}, i=1,2$, we only have the restriction on the failure rate functions $r_{T_{i}}\left(x_{i}\right)+r_{T_{3}}\left(x_{3}\right)=\lambda_{X_{i}}$. In particular, we will consider $T_{i}$ exponentially distributed with parameter $\lambda_{i}, i=1,2,3$. This choice has nice properties and will be justified in Theorem 3.7.8.

Remark 3.7.3. Other choices for the distributions of $T_{i}, i=1,2,3$, exist. For example consider the following failure rate functions $r_{T_{1}}(x)=r_{T_{2}}(x)=2+\sin (x)$ and $r_{T_{3}}(x)=1-\sin (x)$ to obtain $\lambda_{X_{i}}=3, i=1,2$. These failure rate functions will be used in Example 3.7.9.

Since the comonotonic copula is an extreme value copula, whenever $\bar{C}_{T_{1}, T_{2}}(u, v)$, the survival copula corresponding to $S_{T_{1}, T_{2}}\left(x_{1}, x_{2}\right)$, is also an extreme value copula and $T_{i}$ are exponentially distributed, $i=1,2,3$, considering the property of bivariate extreme value copulas given in (3.29) and $q>0$ we obtain

$$
\begin{aligned}
S_{X_{1}, X_{2}}\left(q x_{1}, q x_{2}\right) & =\bar{C}_{T_{1}, T_{2}}\left(\exp \left\{-\lambda_{1} q x_{1}\right\}, \exp \left\{-\lambda_{2} q x_{2}\right\}\right) \min \left[\exp \left\{-\lambda_{3} q x_{1}\right\}, \exp \left\{-\lambda_{3} q x_{2}\right\}\right] \\
& =\left\{\bar{C}_{T_{1}, T_{2}}\left(\exp \left\{-\lambda_{1} x_{1}\right\}, \exp \left\{-\lambda_{2} x_{2}\right\}\right)\right\}^{q}\left\{\min \left[\exp \left\{-\lambda_{3} x_{1}\right\}, \exp \left\{-\lambda_{3} x_{2}\right\}\right]\right\}^{q} \\
& =\left\{S_{X_{1}, X_{2}}\left(x_{1}, x_{2}\right)\right\}^{q} .
\end{aligned}
$$

Thus, relation (3.33) holds true and $S_{X_{1}, X_{2}}\left(x_{1}, x_{2}\right)$ is an extreme value survival function with exponential marginals. In fact, we proved the following lemma.

Lemma 3.7.4. If $S_{T_{1}, T_{2}}\left(x_{1}, x_{2}\right)$ is an extreme value survival function in (3.2) with exponential marginals and $T_{3}$ is exponentially distributed, then $S_{X_{1}, X_{2}}\left(x_{1}, x_{2}\right)$ is an extreme value EMO model with exponential marginals.

The next two lemmas relate the distributions of $\left(T_{1}, T_{2}\right), T_{3}$ and $\left(X_{1}, X_{2}\right)$ in a similar way.

Lemma 3.7.5. If $S_{X_{1}, X_{2}}\left(x_{1}, x_{2}\right)$ is an extreme value survival function with exponential marginals in (3.2) and $T_{3}$ is exponentially distributed, then $S_{T_{1}, T_{2}}\left(x_{1}, x_{2}\right)$ is an extreme value survival function with exponential marginals. 
Proof. From the general expression for survival function of EMO models (3.2), let $x_{3-i}=0$ to obtain $S_{X_{i}}\left(x_{i}\right)=S_{T_{i}}\left(x_{i}\right) S_{T_{3}}\left(x_{i}\right)$. Once $X_{i}$ and $T_{3}$ are exponentially distributed, we conclude that $T_{i}$ are exponentially distributed, $i=1,2$. Since $S_{X_{1}, X_{2}}\left(x_{1}, x_{2}\right)$ is an extreme value survival function with exponential marginals, then relation (3.33) holds true and considering again representation (3.2) with $q>0$

$$
\begin{aligned}
S_{X_{1}, X_{2}}\left(q x_{1}, q x_{2}\right) & =S_{T_{1}, T_{2}}\left(q x_{1}, q x_{2}\right) S_{T_{3}}\left(\max \left(q x_{1}, q x_{2}\right)\right) \\
& =\left[S_{X_{1}, X_{2}}\left(x_{1}, x_{2}\right)\right]^{q}=\left[S_{T_{1}, T_{2}}\left(x_{1}, x_{2}\right) S_{T_{3}}\left(\max \left(x_{1}, x_{2}\right)\right)\right]^{q},
\end{aligned}
$$

But $T_{3}$ is exponentially distributed, so that $S_{T_{3}}\left(\max \left(q x_{1}, q x_{2}\right)\right)=\left[S_{T_{3}}\left(\max \left(x_{1}, x_{2}\right)\right)\right]^{q}$, and we conclude $S_{T_{1}, T_{2}}\left(q x_{1}, q x_{2}\right)=\left[S_{T_{1}, T_{2}}\left(x_{1}, x_{2}\right)\right]^{q}$, finishing the proof.

Lemma 3.7.6. If $S_{T_{1}, T_{2}}\left(x_{1}, x_{2}\right)$ and $S_{X_{1}, X_{2}}\left(x_{1}, x_{2}\right)$ are extreme value survival functions with exponential marginals in (3.2), then $T_{3}$ is exponentially distributed.

Proof. Follow similar steps as in the proof of Lemma 3.7.5.

With the additional assumption of absolutely continuity of the joint survival function of $\left(T_{1}, T_{2}\right)$, in the next theorem we justify the particular choice of $T_{i}, i=1,2,3$, to be exponentially distributed in order to obtain extreme value EMO models with exponential marginals in (3.34).

Theorem 3.7.7. If the joint survival function of $\left(T_{1}, T_{2}\right)$ is absolutely continuous and $S_{X_{1}, X_{2}}\left(x_{1}, x_{2}\right)$ is an extreme value survival function with exponential marginals in (3.2) then $S_{T_{1}, T_{2}}\left(x_{1}, x_{2}\right)$ is an extreme value survival function with exponential marginals and $T_{3}$ is exponentially distributed.

Proof. Suppose $x_{1}>x_{2} \geq 0$ and $q>0$. Since $S_{X_{1}, X_{2}}\left(x_{1}, x_{2}\right)$ is an extreme value survival function with exponential marginals, then relation (3.33) holds true. Moreover, taking into account the general expression for survival function of EMO models (3.2) and the exponential representation for bivariate survival functions (3.3), for $q>0$ we have

$$
\begin{aligned}
S_{X_{1}, X_{2}}\left(q x_{1}, q x_{2}\right) & =\exp \left\{-H_{T_{1}}\left(q x_{1}\right)-H_{T_{3}}\left(q x_{1}\right)-H_{T_{2}}\left(q x_{2}\right)+H_{T_{1}, T_{2}}\left(q x_{1}, q x_{2}\right)\right\} \\
& =\left[S_{X_{1}, X_{2}}\left(x_{1}, x_{2}\right)\right]^{q}=\exp \left\{-q H_{T_{1}}\left(x_{1}\right)-q H_{T_{3}}\left(x_{1}\right)-q H_{T_{2}}\left(x_{2}\right)+q H_{T_{1}, T_{2}}\left(x_{1}, x_{2}\right)\right\} .
\end{aligned}
$$

Taking logarithms in both sides and calculating the mixed partial derivatives, we get

$$
q \frac{\partial^{2}}{\partial x_{1} \partial x_{2}} H_{T_{1}, T_{2}}\left(q x_{1}, q x_{2}\right)=\frac{\partial^{2}}{\partial x_{1} \partial x_{2}} H_{T_{1}, T_{2}}\left(x_{1}, x_{2}\right) .
$$

Integrating the previous expression we have

$$
\int_{0}^{x_{1}} \int_{0}^{x_{2}} q \frac{\partial^{2}}{\partial u \partial v} H_{T_{1}, T_{2}}(q u, q v) \mathrm{d} v \mathrm{~d} u=\int_{0}^{x_{1}} \int_{0}^{x_{2}} \frac{\partial^{2}}{\partial u \partial v} H_{T_{1}, T_{2}}(u, v) \mathrm{d} v \mathrm{~d} u .
$$

The boundary conditions $H_{T_{1}, T_{2}}\left(x_{1}, 0\right)=H_{T_{1}, T_{2}}\left(0, x_{2}\right)=0$ imply the following relations for partial derivatives $\frac{\partial}{\partial x_{1}} H_{T_{1}, T_{2}}\left(x_{1}, 0\right)=\frac{\partial}{\partial x_{2}} H_{T_{1}, T_{2}}\left(0, x_{2}\right)=0, x_{1}, x_{2} \geq 0$. Thus we arrive to the functional equation

$$
H_{T_{1}, T_{2}}\left(q x_{1}, q x_{2}\right)=q H_{T_{1}, T_{2}}\left(x_{1}, x_{2}\right) .
$$


Since $S_{X_{1}, X_{2}}\left(x_{1}, x_{2}\right)$ is an extreme value survival function with exponential marginals, from (3.33) and $q>0$,

$$
\left[\frac{S_{X_{1}, X_{2}}\left(x_{1}, x_{2}\right)}{S_{X_{1}}\left(x_{1}\right) S_{X_{2}}\left(x_{2}\right)}\right]^{q}=\frac{S_{X_{1}, X_{2}}\left(q x_{1}, q x_{2}\right)}{S_{X_{1}}\left(q x_{1}\right) S_{X_{2}}\left(q x_{2}\right)} .
$$

Applying the exponential representation for bivariate survival functions (3.3) to (3.36) and considering (3.35) we obtain the functional equation $H_{T_{3}}\left(q x_{2}\right)=q H_{T_{3}}\left(x_{2}\right)$, for all $x_{2}, q>0$. Denoting $\lambda_{3}=H_{T_{3}}(1)$ we have the general solution $H_{T_{3}}\left(x_{2}\right)=\lambda_{3} x_{2}$. Since $H_{T_{3}}\left(x_{2}\right)$ is nonnegative then $\lambda_{3}>0$. The conclusion is the same when $x_{2} \geq x_{1} \geq 0$. Thus, $T_{3}$ is exponentially distributed with parameter $\lambda_{3}$. Applying Lemma 3.7.5 we conclude the proof.

From Theorem 3.7.7 and Lemma 3.7.4 we obtain the following characterization statement.

Theorem 3.7.8. Let the joint survival function of $\left(T_{1}, T_{2}\right)$ be absolutely continuous. Then $S_{X_{1}, X_{2}}\left(x_{1}, x_{2}\right)$ in (3.2) is an extreme value EMO model with exponential marginals if and only if $S_{T_{1}, T_{2}}\left(x_{1}, x_{2}\right)$ is an extreme value survival function with exponential marginals and $T_{3}$ is exponentially distributed.

Relaxing the assumption of absolutely continuity of the survival function $S_{T_{1}, T_{2}}\left(x_{1}, x_{2}\right)$, it is possible to obtain an extreme value EMO model where $T_{i}, i=1,2,3$, are not exponentially distributed, as shown in the next example.

Example 3.7.9 (Extreme value EMO model function with non-exponentially distributed $T_{i}$ ). The Marshall-Olkin distribution is an example of extreme value survival function as well as of EMO model. In Example 3.6.1 we obtained the Marshall-Olkin survival function in a two step construction, first using a GMO model and then an EMO model. In particular, in the second step we considered nonnegative continuous random variables $\left(T_{1}, T_{2}\right)$ with survival function $S_{T_{1}, T_{2}}\left(x_{1}, x_{2}\right)=\exp \left\{-x_{1}-\right.$ $\left.x_{2}-H_{Y_{3}}\left(\max \left(x_{1}, x_{2}\right)\right)\right\}$, where $H_{Y_{3}}(x)=x-\cos (x)+1$. Then we took $T_{3}$ independent of $\left(T_{1}, T_{2}\right)$ with cumulative failure rate $H_{T_{3}}(x)=x+\cos (x)-1$ to obtain $\left(X_{1}, X_{2}\right)=\left(\min \left(T_{1}, T_{3}\right), \min \left(T_{2}, T_{3}\right)\right)$ with Marshall-Olkin survival function $S_{X_{1}, X_{2}}\left(x_{1}, x_{2}\right)=\exp \left\{-x_{1}-x_{2}-2 \max \left(x_{1}, x_{2}\right)\right\}$. Notice that $S_{T_{1}, T_{2}}\left(x_{1}, x_{2}\right)$ is neither an extreme value survival function nor absolutely continuous, as well as $T_{3}$ is not exponentially distributed. See also Remark 3.7.3.

Remark 3.7.10. In Example 3.7.9, the survival $S_{T_{1}, T_{2}}\left(x_{1}, x_{2}\right)$ has a singular component with support on the set $\Omega=\left\{\left(x_{1}, x_{2}\right) \in \mathbb{R}_{+}^{2} \mid x_{1}=x_{2}=x\right\}$.

Remark 3.7.11. Notice that the functional equation (3.35) on dependence function $H_{T_{1}, T_{2}}\left(x_{1}, x_{2}\right)$, being homogenous of order 1, can be used to characterize bivariate extreme value survival functions $S_{T_{1}, T_{2}}\left(x_{1}, x_{2}\right)$ with exponential marginals, even when absolute continuity does not hold true, see Theorem 6.2 in Joe (1997).

We deduce the following characterization in the general case.

Lemma 3.7.12. A bivariate survival function with exponential marginals is extreme value if an only if its dependence function is homogeneous of order 1.

To characterize the extreme value EMO models with exponential marginals when the joint survival function of $\left(T_{1}, T_{2}\right)$ is not absolutely continuous we will need and an additional assumption. It is given below. 
Theorem 3.7.13. Let $X_{i}$ be exponentially distributed, $i=1,2$. Then $S_{X_{1}, X_{2}}\left(x_{1}, x_{2}\right)$ in (3.2) is an extreme value EMO model if and only if for all $q>0$,

$$
H_{T_{1}, T_{2}}\left(q x_{1}, q x_{2}\right)+H_{T_{3}}\left(q \min \left(x_{1}, x_{2}\right)\right)=q H_{T_{1}, T_{2}}\left(x_{1}, x_{2}\right)+q H_{T_{3}}\left(\min \left(x_{1}, x_{2}\right)\right) .
$$

Proof. Observe that under the stated conditions the dependence function for the EMO model can be obtained from (3.5) as $H_{X_{1}, X_{2}}\left(x_{1}, x_{2}\right)=H_{T_{1}, T_{2}}\left(x_{1}, x_{2}\right)+H_{T_{3}}\left(\min \left(x_{1}, x_{2}\right)\right)$. To finish the proof, just apply Lemma 3.7.12.

Given the result obtained in Theorem 3.7.8, in the sequel we consider absolutely continuous survival function for $\left(T_{1}, T_{2}\right)$ so that we have $T_{i}$ exponentially distributed with parameter $\lambda_{i}, i=$ $1,2,3$.

In the next theorem we obtain the general expressions of the survival function, survival copula and Pickands measure for extreme value EMO models.

Theorem 3.7.14. Suppose $S_{X_{1}, X_{2}}\left(x_{1}, x_{2}\right)$ given by (3.2) is an extreme value survival function with exponential marginals and the joint survival function of $\left(T_{1}, T_{2}\right)$ is absolutely continuous. Then, the survival function of $\left(X_{1}, X_{2}\right)$ is

$$
\begin{aligned}
S_{X_{1}, X_{2}}\left(x_{1}, x_{2}\right) & =\exp \left\{-\left(\lambda_{1} x_{1}+\lambda_{2} x_{2}\right) \mathcal{A}_{T_{1}, T_{2}}\left(\frac{\lambda_{1} x_{1}}{\lambda_{1} x_{1}+\lambda_{2} x_{2}}\right)\right\} \\
& \times \exp \left\{-\lambda_{3} \max \left(x_{1}, x_{2}\right)\right\}
\end{aligned}
$$

where $\mathcal{A}_{T_{1}, T_{2}}(w)$ is the Pickands dependence function corresponding to $\bar{C}_{T_{1}, T_{2}}(u, v)$ and $\lambda_{i}>0$, $i=1,2,3$. The survival copula of the bivariate extreme value EMO model, is given by

$$
\begin{aligned}
& \bar{C}_{X_{1}, X_{2}}(u, v)= \\
& =\exp \left\{(\ln u v)\left[\left(\frac{\lambda_{1} w}{\lambda_{1}+\lambda_{3}}+\frac{\lambda_{2}(1-w)}{\lambda_{2}+\lambda_{3}}\right) \mathcal{A}_{T_{1}, T_{2}}\left(\frac{\frac{\lambda_{1} w}{\lambda_{1}+\lambda_{3}}}{\frac{\lambda_{1} w}{\lambda_{1}+\lambda_{3}}+\frac{\lambda_{2}(1-w)}{\lambda_{2}+\lambda_{3}}}\right)+\max \left(\frac{\lambda_{3} w}{\lambda_{1}+\lambda_{3}}, \frac{\lambda_{3}(1-w)}{\lambda_{2}+\lambda_{3}}\right)\right]\right\},
\end{aligned}
$$

where $u, v \in(0,1)$ and $w=\frac{\ln u}{\ln u v}$. The Pickands measure $\mathbb{H}$ is given by

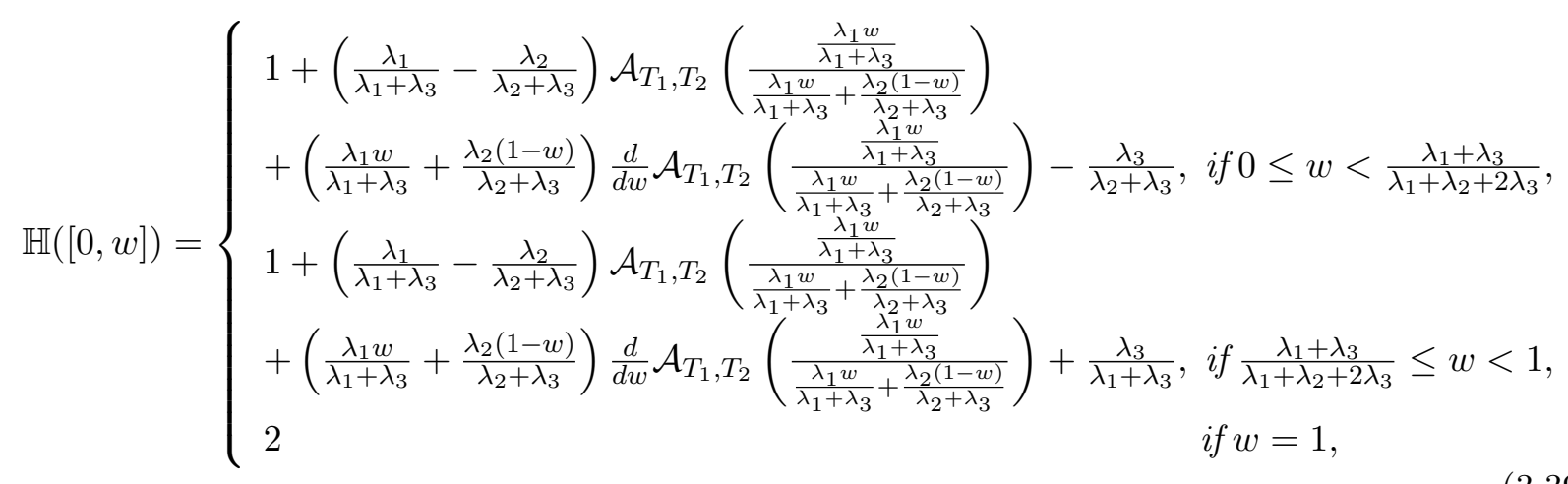

where $\frac{d}{d w} \mathcal{A}_{T_{1}, T_{2}}($.$) refers to the right-hand derivative.$

Proof. From Theorem 3.7.7, $T_{3}$ is exponentially distributed and $S_{T_{1}, T_{2}}\left(x_{1}, x_{2}\right)$ is an extreme value survival function with exponential marginals. Denote by $\lambda_{i}=\frac{1}{E\left(T_{i}\right)}>0, i=1,2,3$. From Sklar's Theorem, see Theorem 2.2.3 in Nelsen (2006), and the expression for bivariate copulas written in 
terms of Pickands dependence function (3.30) we have

$$
\begin{aligned}
S_{T_{1}, T_{2}}\left(x_{1}, x_{2}\right) & =\bar{C}_{T_{1}, T_{2}}\left(S_{T_{1}}\left(x_{1}\right), S_{T_{2}}\left(x_{2}\right)\right)=\bar{C}_{T_{1}, T_{2}}\left(\exp \left\{-\lambda_{1} x_{1}\right\}, \exp \left\{-\lambda_{2} x_{2}\right\}\right) \\
& =\exp \left\{-\left(\lambda_{1} x_{1}+\lambda_{2} x_{2}\right) \mathcal{A}_{T_{1}, T_{2}}\left(\frac{\lambda_{1} x_{1}}{\lambda_{1} x_{1}+\lambda_{2} x_{2}}\right)\right\}
\end{aligned}
$$

Considering the expression for EMO survival functions (3.1) we obtain relation (3.37), i.e., the survival function of $\left(X_{1}, X_{2}\right)$.

Let $u=S_{X_{1}}\left(x_{1}\right)=\exp \left\{-\left(\lambda_{1}+\lambda_{3}\right) x_{1}\right\}$ and $v=S_{X_{2}}\left(x_{2}\right)=\exp \left\{-\left(\lambda_{2}+\lambda_{3}\right) x_{2}\right\}$. From Sklar's Theorem, $\bar{C}_{X_{1}, X_{2}}(u, v)=S_{X_{1}, X_{2}}\left(\frac{-\ln u}{\lambda_{1}+\lambda_{3}}, \frac{-\ln v}{\lambda_{2}+\lambda_{3}}\right)$. Applying relation (3.31), we have the Pickands dependence function given by $\mathcal{A}_{X_{1}, X_{2}}(w)=-\ln S_{X_{1}, X_{2}}\left(\frac{w}{\lambda_{1}+\lambda_{3}}, \frac{1-w}{\lambda_{2}+\lambda_{3}}\right)$. Substituting $S_{X_{1}, X_{2}}\left(x_{1}, x_{2}\right)$ by its expression given by (3.37) we obtain

$$
\begin{aligned}
\mathcal{A}_{X_{1}, X_{2}}(w) & =\left(\frac{\lambda_{1} w}{\lambda_{1}+\lambda_{3}}+\frac{\lambda_{2}(1-w)}{\lambda_{2}+\lambda_{3}}\right) \mathcal{A}_{T_{1}, T_{2}}\left(\frac{\frac{\lambda_{1} w}{\lambda_{1}+\lambda_{3}}}{\frac{\lambda_{1} w}{\lambda_{1}+\lambda_{3}}+\frac{\lambda_{2}(1-w)}{\lambda_{2}+\lambda_{3}}}\right) \\
& +\max \left(\frac{\lambda_{3} w}{\lambda_{1}+\lambda_{3}}, \frac{\lambda_{3}(1-w)}{\lambda_{2}+\lambda_{3}}\right) .
\end{aligned}
$$

Considering again expression for extreme value copulas (3.30) along with relation (3.40), we obtain (3.38), i.e., the survival copula of the bivariate extreme value EMO model. By its turn, from the expression of Pickands measure in terms of the derivative of Pickands dependence function (3.32) and the previous relation (3.40) we obtain (3.39), the Pickands measure $\mathbb{H}$.

Remark 3.7.15. The point masses of $\mathbb{H}$ at $w=0$ and $w=1$ are given by

$$
\mathbb{H}(\{0\})=1+\frac{d}{d w} \mathcal{A}_{X_{1}, X_{2}}(0) \quad \text { and } \quad \mathbb{H}(\{1\})=1-\frac{d}{d w} \mathcal{A}_{X_{1}, X_{2}}(1),
$$

where $\frac{d}{d w} \mathcal{A}_{X_{1}, X_{2}}(1)=\sup _{0 \leq w<1} \frac{d}{d w} \mathcal{A}_{X_{1}, X_{2}}(w)$.

Remark 3.7.16. Whenever the derivative of $\mathcal{A}_{T_{1}, T_{2}}(w)$ is continuous, for all the copulas satisfying (3.39) we have

$$
\mathbb{H}\left(\left\{\frac{\lambda_{1}+\lambda_{3}}{\lambda_{1}+\lambda_{2}+2 \lambda_{3}}\right\}\right)=\frac{\lambda_{3}}{\lambda_{1}+\lambda_{3}}+\frac{\lambda_{3}}{\lambda_{2}+\lambda_{3}} .
$$

We apply relations established in Theorem 3.7.14 in the next two examples.

Example 3.7.17 (Marshall-Olkin survival function). Consider the Marshall-Olkin survival function given in (2.4),

$$
S_{X_{1}, X_{2}}\left(x_{1}, x_{2}\right)=\exp \left\{-\lambda_{1} x_{1}-\lambda_{2} x_{2}-\lambda_{3} \max \left(x_{1}, x_{2}\right)\right\},
$$

where $\lambda_{i}>0, i=1,2,3$. In this case, $\bar{C}_{T_{1}, T_{2}}(u, v)=u v$, i.e., the independence copula. Therefore, $\mathcal{A}_{T_{1}, T_{2}}(w)=1, w \in[0,1]$. From (3.40) the Pickands dependence function is

$$
\begin{aligned}
\mathcal{A}_{X_{1}, X_{2}}(w) & =\frac{\lambda_{1} w}{\lambda_{1}+\lambda_{3}}+\frac{\lambda_{2}(1-w)}{\lambda_{2}+\lambda_{3}}+\max \left(\frac{\lambda_{3} w}{\lambda_{1}+\lambda_{3}}, \frac{\lambda_{3}(1-w)}{\lambda_{2}+\lambda_{3}}\right) \\
& = \begin{cases}1-\frac{\lambda_{3} w}{\lambda_{1}+\lambda_{3}}, & \text { if } 0 \leq w<\frac{\lambda_{1}+\lambda_{3}}{\lambda_{1}+\lambda_{2}+2 \lambda_{3}} \\
\frac{\lambda_{2}}{\lambda_{2}+\lambda_{3}}+\frac{\lambda_{3} w}{\lambda_{2}+\lambda_{3}}, & \text { if } \frac{\lambda_{1}+\lambda_{3}}{\lambda_{1}+\lambda_{2}+2 \lambda_{3}} \leq w \leq 1 .\end{cases}
\end{aligned}
$$


Considering that $w=\frac{\ln u}{\ln u v}, u, v$ in $(0,1), \mathcal{A}_{X_{1}, X_{2}}(w)$ can be presented as

$$
\mathcal{A}_{X_{1}, X_{2}}(u, v)= \begin{cases}1-\frac{\lambda_{3}}{\lambda_{1}+\lambda_{3}} \frac{\ln u}{\ln u v}, & \text { if } 1>u^{\frac{\lambda_{3}}{\lambda_{1}+\lambda_{3}}}>v^{\frac{\lambda_{3}}{\lambda_{2}+\lambda_{3}}}>0 \\ \frac{\lambda_{2}}{\lambda_{2}+\lambda_{3}}+\frac{\lambda_{3}}{\lambda_{2}+\lambda_{3}} \frac{\ln u}{\ln u v}, & \text { if } 0<u^{\frac{\lambda_{3}}{\lambda_{1}+\lambda_{3}}} \leq v^{\frac{\lambda_{3}}{\lambda_{2}+\lambda_{3}}}<1 .\end{cases}
$$

From (3.38), the Marshall-Olkin survival copula writes as

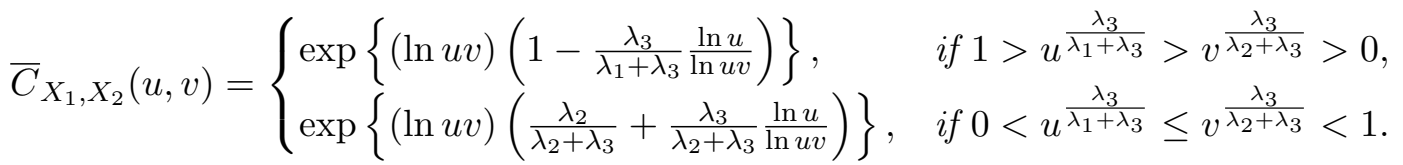

This survival copula can be simplified to

$$
\bar{C}_{X_{1}, X_{2}}(u, v)= \begin{cases}u^{\frac{\lambda_{1}}{\lambda_{1}+\lambda_{3}}} v, & \text { if } 1>u^{\frac{\lambda_{3}}{\lambda_{1}+\lambda_{3}}}>v^{\frac{\lambda_{3}}{\lambda_{2}+\lambda_{3}}}>0 \\ u v^{\frac{\lambda_{2}}{\lambda_{2}+\lambda_{3}}}, \quad \text { if } 0<u^{\frac{\lambda_{3}}{\lambda_{1}+\lambda_{3}}} \leq v^{\frac{\lambda_{3}}{\lambda_{2}+\lambda_{3}}}<1\end{cases}
$$

From (3.39) the Pickands measure is

$$
\mathbb{H}([0, w])= \begin{cases}\frac{\lambda_{1}}{\lambda_{1}+\lambda_{3}}, & \text { if } 0 \leq w<\frac{\lambda_{1}+\lambda_{3}}{\lambda_{1}+\lambda_{2}+2 \lambda_{3}}, \\ 2-\frac{\lambda_{2}}{\lambda_{2}+\lambda_{3}}, & \text { if } \frac{\lambda_{1}+\lambda_{3}}{\lambda_{1}+\lambda_{2}+2 \lambda_{3}} \leq w<1, \\ 2, & \text { if } w=1 .\end{cases}
$$

Therefore, in the Marshall-Olkin case the Pickands measure

$$
\mathbb{H}(\{w\})= \begin{cases}\frac{\lambda_{1}}{\lambda_{1}+\lambda_{3}}, & \text { if } w=0, \\ \frac{\lambda_{3}}{\lambda_{1}+\lambda_{3}}+\frac{\lambda_{3}}{\lambda_{2}+\lambda_{3}}, & \text { if } w=\frac{\lambda_{1}+\lambda_{3}}{\lambda_{1}+\lambda_{2}+2 \lambda_{3}}, \\ \frac{\lambda_{2}}{\lambda_{2}+\lambda_{3}}, & \text { if } w=1,\end{cases}
$$

is a discrete measure whose support on the two-dimensional simplex consists of three points, a result already obtained by Mai and Scherer (2010).

Example 3.7.18 (EMO extreme value survival function where the dependence structure of $\left(T_{1}, T_{2}\right)$ is represented by Gumbel-Hougaard survival copula). Consider the Gumbel's type III bivariate exponential survival function with unit exponential marginals and survival function (2.3),

$$
S\left(x_{1}, x_{2}\right)=\exp \left\{-\left(x_{1}^{m}+x_{2}^{m}\right)^{\frac{1}{m}}\right\}
$$

where $m \geq 1$, and its corresponding survival copula

$$
\bar{C}(u, v)=\exp \left\{-\left[(-\ln u)^{m}+(-\ln v)^{m}\right]^{\frac{1}{m}}\right\},
$$

also known as Gumbel (family B6 in Joe (1997)), Gumbel-Hougaard (equation (2.30) in Balakrishnan and Lai (2009)) or logistic survival copula (in Tawn (1988)). Since this is an example of bivariate extreme value copula, choose $T_{i}$ exponentially distributed with parameter $\lambda_{i}, i=1,2$, and consider the Gum- 
bel's Type III bivariate exponential survival function for $\left(T_{1}, T_{2}\right)$ given by (3.12), i.e.,

$$
S_{T_{1}, T_{2}}\left(x_{1}, x_{2}\right)=\exp \left\{-\left[\left(\lambda_{1} x_{1}\right)^{m}+\left(\lambda_{2} x_{2}\right)^{m}\right]^{\frac{1}{m}}\right\}
$$

Choosing $T_{3}$ exponentially distributed, independent of $\left(T_{1}, T_{2}\right)$ and considering the expression of EMO survival function (3.1), we obtain the following bivariate extreme value EMO survival function

$$
S_{X_{1}, X_{2}}\left(x_{1}, x_{2}\right)=\exp \left\{-\left[\left(\lambda_{1} x_{1}\right)^{m}+\left(\lambda_{2} x_{2}\right)^{m}\right]^{\frac{1}{m}}\right\} \exp \left\{-\lambda_{3} \max \left(x_{1}, x_{2}\right)\right\} .
$$

From (3.31), the Pickands dependence function for $\left(T_{1}, T_{2}\right)$ is

$$
\mathcal{A}_{T_{1}, T_{2}}(w)=\left[w^{m}+(1-w)^{m}\right]^{\frac{1}{m}}
$$

and from (3.40), the Pickands dependence function for $\left(X_{1}, X_{2}\right)$ is

$$
\mathcal{A}_{X_{1}, X_{2}}(w)=\left[\left(\frac{\lambda_{1} w}{\lambda_{1}+\lambda_{3}}\right)^{m}+\left(\frac{\lambda_{2}(1-w)}{\lambda_{2}+\lambda_{3}}\right)^{m}\right]^{\frac{1}{m}}+\max \left(\frac{\lambda_{3} w}{\lambda_{1}+\lambda_{3}}, \frac{\lambda_{3}(1-w)}{\lambda_{2}+\lambda_{3}}\right) .
$$

Applying relation (3.39), we obtain the Pickands measure as

$$
\mathbb{H}([0, w])= \begin{cases}1-\frac{\lambda_{3}}{\lambda_{2}+\lambda_{3}}+\left[\left(\frac{\lambda_{1} w}{\lambda_{1}+\lambda_{3}}\right)^{m}+\left(\frac{\lambda_{2}(1-w)}{\lambda_{2}+\lambda_{3}}\right)^{m}\right]^{\frac{1}{m}-1} & \\ \times\left[\left(\frac{\lambda_{1}}{\lambda_{1}+\lambda_{3}}\right)^{m} w^{m-1}-\left(\frac{\lambda_{2}}{\lambda_{2}+\lambda_{3}}\right)^{m}(1-w)^{m-1}\right], & \text { if } 0 \leq w<\frac{\lambda_{1}+\lambda_{3}}{\lambda_{1}+\lambda_{2}+2 \lambda_{3}}, \\ 1+\frac{\lambda_{3}}{\lambda_{1}+\lambda_{3}}+\left[\left(\frac{\lambda_{1} w}{\lambda_{1}+\lambda_{3}}\right)^{m}+\left(\frac{\lambda_{2}(1-w)}{\lambda_{2}+\lambda_{3}}\right)^{m}\right]^{\frac{1}{m}-1} & \\ \times\left[\left(\frac{\lambda_{1}}{\lambda_{1}+\lambda_{3}}\right)^{m} w^{m-1}-\left(\frac{\lambda_{2}}{\lambda_{2}+\lambda_{3}}\right)^{m}(1-w)^{m-1}\right], & \text { if } \frac{\lambda_{1}+\lambda_{3}}{\lambda_{1}+\lambda_{2}+2 \lambda_{3}} \leq w<1, \\ 2, & \text { if } w=1 .\end{cases}
$$

Applying relation (3.41) in Remark 3.7.16

$$
\mathbb{H}\left(\left\{\frac{\lambda_{1}+\lambda_{3}}{\lambda_{1}+\lambda_{2}+2 \lambda_{3}}\right\}\right)=\frac{\lambda_{3}}{\lambda_{1}+\lambda_{3}}+\frac{\lambda_{3}}{\lambda_{2}+\lambda_{3}}
$$

Notice that when $m=1$, the Gumbel survival copula becomes the independence copula and we obtain the results of Example 3.7.17. For $1<m<\infty$, the remaining mass, $2-\left(\frac{\lambda_{3}}{\lambda_{1}+\lambda_{3}}+\frac{\lambda_{3}}{\lambda_{2}+\lambda_{3}}\right)$, is spread over the interval $\left[0, \frac{\lambda_{1}+\lambda_{3}}{\lambda_{1}+\lambda_{2}+2 \lambda_{3}}\right) \bigcup\left(\frac{\lambda_{1}+\lambda_{3}}{\lambda_{1}+\lambda_{2}+2 \lambda_{3}}, 1\right]$ and, in particular, $\mathbb{H}(\{0\})=\mathbb{H}(\{1\})=0$.

\subsection{Bayesian data analysis with EMO models}

Presented in Section 3.5, expression (3.18) provides the joint survival function of EMO models decomposed in absolutely continuous and singular components. By its turn, the density function of EMO models is given by

$$
f_{X_{1}, X_{2}}\left(x_{1}, x_{2}\right)= \begin{cases}f_{T_{1}, T_{2}}\left(x_{1}, x_{2}\right) S_{T_{3}}\left(x_{1}\right)-\frac{\partial}{\partial x_{2}} S_{T_{1}, T_{2}}\left(x_{1}, x_{2}\right) f_{T_{3}}\left(x_{1}\right) & \text { if } x_{1}>x_{2} \geq 0 \\ f_{T_{3}}(x) S_{T_{1}, T_{2}}(x, x) & \text { if } x_{1}=x_{2}=x \geq 0 \\ f_{T_{1}, T_{2}}\left(x_{1}, x_{2}\right) S_{T_{3}}\left(x_{2}\right)-\frac{\partial}{\partial x_{1}} S_{T_{1}, T_{2}}\left(x_{1}, x_{2}\right) f_{T_{3}}\left(x_{2}\right) & \text { if } 0 \leq x_{1}<x_{2} .\end{cases}
$$




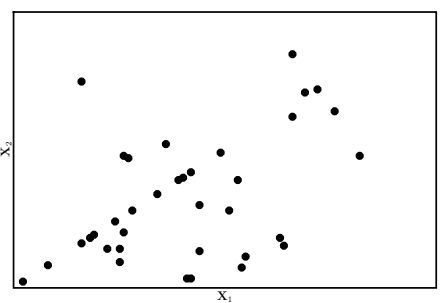

Figure 3.4: UEFA Champion's League data-Meintanis (2007)

In the EMO survival function (3.2), let us fix $T_{3}$ to be exponentially distributed, i.e., $S_{T_{3}}(x)=$ $\exp \{-\lambda x\}, \lambda>0$, to obtain

$$
S_{X_{1}, X_{2}}\left(x_{1}, x_{2}\right)=S_{T_{1}, T_{2}}\left(x_{1}, x_{2}\right) \exp \left\{-\lambda \max \left(x_{1}, x_{2}\right)\right\} .
$$

In this Section we consider the survival function (3.44) to illustrate how EMO models can be used to analyse bivariate data sets with a singular component along the main diagonal. Bayesian estimation of the parameters are performed using the software OpenBugs.

We selected the data presented in Meintanis (2007) to illustrate the procedure. In a football (soccer) data set of UEFA Champion's League, Meintanis (2007) considered matches where (i) there was at least one goal scored by the home team, and (ii) there was at least one goal scored directly from a kick (penalty kick, foul kick, or other kick) by any team. Let $X_{1}$ be the time (in minutes) of the first kick goal scored by any team, and $X_{2}$ be the time of the first goal of any type scored by the home team. With this kind of nonnegative continuous data, one may have $X_{1}<X_{2}$, $X_{1}>X_{2}$ or $X_{1}=X_{2}$, see Figure 3.4.

Five models are considered in the analysis of UEFA Champion's League data set: two of them are absolutely continuous and three belong to the class EMO (exhibiting a singular component along the main diagonal $x_{1}=x_{2}=x \geq 0$ ), with survival function given by (3.44).

The absolutely continuous models are Gumbel (1960) type I bivariate exponential and the bivariate exponential distribution of Block and Basu (1974):

Table 3.1: Absolutely continuous survival functions for lifetimes $\left(X_{1}, X_{2}\right)$

\begin{tabular}{|c|c|c|}
\hline Model & Description & $S_{X_{1}, X_{2}}\left(x_{1}, x_{2}\right)$ \\
\hline (M1) & Gumbel (1960) type I & $\exp \left\{-\left[\lambda_{1} x_{1}+\lambda_{2} x_{2}+\theta \lambda_{1} \lambda_{2} x_{1} x_{2}\right]\right\}$ \\
\hline (M2) & Block and Basu (1974) & $\begin{array}{l}\frac{\lambda_{1}+\lambda_{2}+\lambda_{12}}{\lambda_{1}+\lambda_{2}} \exp \left\{-\left[\lambda_{1} x_{1}+\lambda_{2} x_{2}+\lambda_{12} \max \left(x_{1}, x_{2}\right)\right]\right\} \\
-\frac{\lambda_{12}}{\lambda_{1}+\lambda_{2}} \exp \left\{-\left[\left(\lambda_{1}+\lambda_{2}+\lambda_{12}\right) \max \left(x_{1}, x_{2}\right)\right]\right\}\end{array}$ \\
\hline
\end{tabular}

where $\lambda_{1}, \lambda_{2}, \lambda_{12}>0$ and $\theta \in[0,1]$.

Concerning the models of the class EMO with survival function (3.44), we considered the following options for $S_{T_{1}, T_{2}}\left(x_{1}, x_{2}\right)$ :

Table 3.2: Joint survival functions for lifetimes $\left(T_{1}, T_{2}\right)$

\begin{tabular}{|c|c|c|}
\hline Model & Description & $S_{T_{1}, T_{2}}\left(x_{1}, x_{2}\right)$ \\
\hline (M3) & Marshall and Olkin (1967) & $\exp \left\{-\left[\lambda_{1} x_{1}+\lambda_{2} x_{2}\right]\right\}$ \\
\hline (M4) & EMO-1 & Gumbel (1960) type I \\
\hline (M5) & EMO-2 & Block and Basu (1974) \\
\hline
\end{tabular}

With the previous choices, we use equation (3.43) to obtain the joint density of the EMO models. In addition, we have to adopt the prior distributions for the parameters of the five models. Our 
choices are given in Table 3.3 .

Table 3.3: Prior distributions

\begin{tabular}{|c|c|}
\hline Parameter & Prior \\
\hline$\lambda_{1}, \lambda_{2}$ and $\lambda_{12}$ & Gamma $(0.1,0.1)$ \\
\hline$\lambda$ & Gamma $(1,1)$ \\
\hline$\theta$ & Uniform $[0,1]$ \\
\hline
\end{tabular}

We generated 55.000 samples for the joint posterior distribution of the considered parameters, where the first 5.000 samples are discarded (burn-in sample). Thus we eliminate the effect of the initial values for the parameters of the model. Selecting every $10^{\text {th }}$ simulated Gibbs sample, we obtain a final sample of size 5.000 to get the posterior summaries of interest.

The five models to be compared have different number of parameters. We consider the DIC (Deviance Information Criterion) defined in Spiegelhalter et al. (2002) as

$$
D I C=D(\hat{\theta})+2 n_{D}=2 \bar{D}-D(\hat{\theta})
$$

where $D(\hat{\theta})$ is the deviance evaluated at the posterior mean $\hat{\theta}=\mathbb{E}[\theta \mid$ data $]$ and $\bar{D}=\mathbb{E}[D(\theta) \mid$ data $]$ is the posterior deviance measuring the quality of the data fit for the model. Note that $n_{D}$ is the effective number of parameters of the model given by $n_{D}=\bar{D}-D(\hat{\theta})$. Smaller value of DIC indicates better model.

The posterior summaries obtained for the five models are summarized in next tables.

Table 3.4: Model (M1) - Gumbel (1960) type I bivariate exponential

\begin{tabular}{|c|c|c|c|}
\hline Parameter & Mean & Sd & 95\% Credible Interval \\
\hline$\lambda_{1}$ & 0.0238 & 0.003964 & $(0.01663,0.03216)$ \\
$\lambda_{2}$ & 0.02935 & 0.004974 & $(0.02038,0.03982)$ \\
$\theta$ & 0.1734 & 0.1588 & $(0.00450,0.5925)$ \\
\hline
\end{tabular}

Table 3.5: Model (M2) - Block and Basu (1974) bivariate exponential

\begin{tabular}{|c|c|c|c|}
\hline Parameter & Mean & Sd & 95\% Credible Interval \\
\hline$\lambda_{1}$ & 0.000094 & 0.000209 & $(4.42 \mathrm{E}-08,0.000669)$ \\
$\lambda_{2}$ & 0.000727 & 0.001383 & $(1.06 \mathrm{E}-06,0.004847)$ \\
$\lambda_{12}$ & 0.045470 & 0.005589 & $(0.03553,0.05784)$ \\
\hline
\end{tabular}

Table 3.6: Model (M3) - Marshall and Olkin (1967) bivariate exponential

\begin{tabular}{|c|c|c|c|}
\hline Parameter & Mean & Sd & 95\% Credible Interval \\
\hline$\lambda_{1}$ & 0.005507 & 0.002521 & $(0.00164,0.01134)$ \\
$\lambda_{2}$ & 0.0172 & 0.00383 & $(0.01049,0.02547)$ \\
$\lambda$ & 0.01926 & 0.003882 & $(0.01224,0.02749)$ \\
\hline
\end{tabular}

Table 3.7: Model (M4): EMO-1 (Gumbel (1960) type I for $\left(T_{1}, T_{2}\right)$ )

\begin{tabular}{|c|c|c|c|}
\hline Parameter & Mean & Sd & 95\% Credible Interval \\
\hline$\lambda_{1}$ & 0.01315 & 0.003003 & $(0.007974,0.01966)$ \\
$\lambda_{2}$ & 0.004985 & 0.002501 & $(0.001329,0.01094)$ \\
$\theta$ & 0.02985 & 0.005555 & $(0.01984,0.02952)$ \\
$\lambda$ & 0.3959 & 0.2801 & $(0.01376,0.9522)$ \\
\hline
\end{tabular}

In Table 3.9 we present the resulting models ordered by increasing DIC value criteria. It can be noticed that EMO models (which take into account singularity along the main diagonal) provided 
Table 3.8: Model (M5) - EMO-2 (Block and Basu (1974) for $\left(T_{1}, T_{2}\right)$ )

\begin{tabular}{|c|c|c|c|}
\hline Parameter & Mean & Sd & 95\% Credible Interval \\
\hline$\lambda_{1}$ & $2.20 \mathrm{E}-04$ & $4.96 \mathrm{E}-04$ & $(2.04 \mathrm{E}-03,0.001639)$ \\
$\lambda_{2}$ & $4.83 \mathrm{E}-05$ & $1.27 \mathrm{E}-04$ & $(2.39 \mathrm{E}-04,3.75 \mathrm{E}-01)$ \\
$\lambda_{12}$ & 0.0332 & 0.005764 & $(0.02273,0.0448)$ \\
$\lambda$ & 0.02034 & 0.004648 & $(0.01269,0.03013)$ \\
\hline
\end{tabular}

a better fit than the absolutely continuous bivariate exponential distributions of Block and Basu (1974) and Gumbel (1960) type I bivariate exponential distributions. Particularly, the model EMO2 (considering Block and Basu (1974) distribution for $\left(T_{1}, T_{2}\right)$ ) presented the best fit according with DIC criteria, followed by model EMO-1 (Gumbel (1960) type I distribution for $\left(T_{1}, T_{2}\right)$ ).

Table 3.9: Monte Carlo estimates for DIC

\begin{tabular}{|c|c|c|}
\hline Model & Description & DIC \\
\hline (M5) & EMO-2 (Block and Basu (1974) for $\left.\left(T_{1}, T_{2}\right)\right)$ & 546.8 \\
(M4) & EMO-1 (Gumbel (1960) type I for $\left(T_{1}, T_{2}\right)$ ) & 584.0 \\
(M3) & Marshall-Olkin (1967) & 600.1 \\
(M2) & Block and Basu (1974) & 633.9 \\
(M1) & Gumbel (1960) type I & 687.0 \\
\hline
\end{tabular}

\subsection{Concluding remarks}

The classical MO model (2.4) finds applications in Reliability, Survival Analysis, Finance and Life Insurance, among other fields. Hence, any extension of this model has its theoretical and practical relevance. In this chapter we extended the MO model (2.4) by assuming dependence between the individual shocks represented by the random variables $T_{1}$ and $T_{2}$ in the stochastic representation (2.9).

The dependence structure in EMO model enlarges the field of applications of MO and GMO distributions allowing one to use the stochastic representation (2.9) even when the assumption of independence between $T_{1}$ and $T_{2}$ is violated. Examples of such situations were provided in Section 3.1.

In Sections 3.2 and 3.3 we studied some probabilistic properties of the extended model, obtained its survival copula representation and investigated the distributional property of residual lifetimes. Quadrant dependence properties, symmetry and asymmetry behaviour and bounds for the extended model were obtained. In spite of the bivariate lack-of-memory property does not hold true for all extended MO models, it was shown that the original vector $\mathbf{X}=\left(\min \left(T_{1}, T_{3}\right), \min \left(T_{2}, T_{3}\right)\right)$ and the vector of residual lifetimes $\mathbf{X}_{t}$ have the same type of survival copula given by (3.9).

In Section 3.4 we provided alternative and inverse survival copula representations for EMO models, which may have a significant effect on analysis of specific probability models relevant to environmental, biological, insurance and finance studies, especially when NQD property naturally holds.

An absolutely continuous version of EMO model that preserves the stochastic representation (2.9) was obtained in Section 3.5. We began Section 3.6 investigating the bivariate LMP in EMO models. Only extended models with singular component have this property. Moreover, it was shown that if $S_{T_{1}, T_{2}}\left(x_{1}, x_{2}\right)$ is absolutely continuous then $\mathbf{X}$ possess the bivariate LMP with exponential marginals if and only if $\left(T_{1}, T_{2}\right)$ possess the bivariate LMP and $T_{i}$ are exponentially distributed, $i=1,2,3$. In the sequel, weak aging properties were studied for the absolutely continuous version 
of the extended model. This version does not possess the weak DFR aging property and conditions for satisfying the weak IFR property were obtained. Then, the bivariate IHR property was analysed and sufficient conditions for obtaining bivariate IHR absolutely continuous extended MO models were established.

In Section 3.7 bivariate extreme value survival copulas that naturally arise from the stochastic representation (2.9) were constructed. Since in the bivariate case the Pickands dependence function as well as the Pickands measure characterize the copula, these expressions were also presented. Considering equation (3.1), the Marshall-Olkin survival copula is obtained. But taking into account relation (3.2), where $T_{1}$ and $T_{2}$ are no more independent, extreme value survival copulas derived from EMO models can be generated taking into account Theorem 3.7.13 and the restriction $r_{T_{i}}\left(x_{i}\right)+$ $r_{T_{3}}\left(x_{i}\right)=\lambda_{X_{i}}, i=1,2$, where $\lambda_{X_{i}}$ are the constant failure rates of the marginals of extreme value EMO model (3.2). In particular, if $S_{T_{1}, T_{2}}\left(x_{1}, x_{2}\right)$ is absolutely continuous then extreme value survival copulas corresponding to EMO models are obtained if and only if the survival copula that joins $\left(T_{1}, T_{2}\right)$ is an extreme value copula and $T_{i}$ are exponentially distributed, $i=1,2,3$.

In Section 3.8 we provided a Bayesian analysis of the data set presented in Meintanis (2007), which displays a singular component along the main diagonal $\left(x_{1}=x_{2}=x \geq 0\right)$. Due to this singularity, EMO distributions are suitable to model such kind of data and, as expected, exhibited a better performance than the two absolutely continuous models considered. For comparison purposes, in the three EMO distributions used in the analysis we fixed the independent common shock $T_{3}$ exponentially distributed. Notice that in the Marshall-Olkin bivariate exponential distribution the only source of dependence between the observed random variables $X_{1}$ and $X_{2}$ comes from this common shock. In Table 3.9 it can be noticed that among the models with singular component, the Marshall-Olkin distribution has the weakest performance. The additional dependence between the individual shocks $T_{1}$ and $T_{2}$ introduced in the two other EMO models has the effect of providing a better fit to the data. It is worth noting that the parameters of EMO models can be also estimated using the maximum likelihood procedure and the density function (3.43). Meanwhile, the complexity of the maximization procedure heavily depends on the functional form of the survival function $S_{T_{1}, T_{2}}\left(x_{1}, x_{2}\right)$, e.g., Model (M5) in Table 3.2.

Block and Basu (1974) obtained an absolutely continuous bivariate exponential distribution from the MO model by removing the singular part in (2.4) and retaining only the absolutely continuous one. The same technique was applied by Kundu and Gupta (2010) in the MO distribution with Weibull exponentials. The obtained models no more possess exponential or Weibull marginal distributions as well as the stochastic representation (2.9) is lost. Meanwhile, they can be used when the observed data does not exhibit ties. It is object of future investigation the properties of absolutely continuous EMO models obtained from this same approach as well as estimation techniques for extended MO models and the multivariate extensions. 


\section{Chapter 4}

\section{Dual extended Marshall-Olkin Model}

\subsection{Introduction}

Fang and Li (2013) introduced a dual version of the Generalized Marshall-Olkin (GMO) model of Li and Pellerey (2011) and studied its properties, motivated by its potential applications in risk analysis, financial engineering and economics. The dual GMO model admits the stochastic representation

$$
\left(X_{1}, X_{2}\right)=\left[\max \left(T_{1}, T_{3}\right), \max \left(T_{2}, T_{3}\right)\right]
$$

where the continuous random variables $T_{i}, i=1,2,3$, with support in $\mathbb{R}=(-\infty, \infty)$ are assumed independent.

Denote by $F_{X_{1}, X_{2}}\left(x_{1}, x_{2}\right)=\mathbb{P}\left(X_{1} \leq x_{1}, X_{2} \leq x_{2}\right)$ the joint distribution function of the random vector $\left(X_{1}, X_{2}\right)$ and by $F_{T_{i}}(x)=\mathbb{P}\left(T_{i} \leq x\right)$ the distribution function of random variables $T_{i}, i=$ $1,2,3$, where $x \in \mathbb{R}$. Then, the joint distribution function of the dual GMO model (4.1) is given by

$$
\begin{aligned}
F_{X_{1}, X_{2}}\left(x_{1}, x_{2}\right) & =\mathbb{P}\left(T_{1} \leq x_{1}, T_{2} \leq x_{2}, T_{3} \leq \min \left\{x_{1}, x_{2}\right\}\right) \\
& =F_{T_{1}}\left(x_{1}\right) F_{T_{2}}\left(x_{2}\right) F_{T_{3}}\left(\min \left\{x_{1}, x_{2}\right\}\right) .
\end{aligned}
$$

Fang and Li (2013) named the model (4.2) "dual", because it is the counterpart of the GMO model based on min instead of max operation in the stochastic representation (4.1). Another difference between GMO model and its dual version is that in GMO model the random variables $T_{i}$ are assumed to be non-negative.

Similarly to the GMO model, its dual version (4.2) can be interpreted in terms of a "fatal shock" model, see Figure 4.1 .

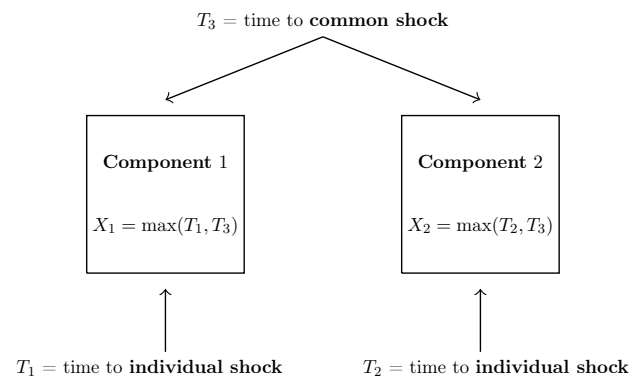

Figure 4.1: "Fatal shock" model in dual generalized Marshall-Olkin distribution. 
It happens that the independence assumption between the individual shocks, although adequate in some situations, may not hold in others, as illustrated in the following examples:

1. An insurance company assigns by (4.1) the loss vector of two insured apartments located in the same building. Each insurance policy covers only the largest loss incurred. Both apartments are subject to common disasters, such as earthquakes or tornados, as well as to individual casualties. The common loss can be considered to have the same value for both apartments. Since they are located in the same neighborhood, some individual casualties, such as thefts, are not supposed to be independent;

2. Let (4.1) represent the time to failure vector of two machines operating in the same factory. Each machine can be fed by two different sources of energy: an individual source and an external one, able to feed both equipment. The machines operate whenever at least one of the sources is properly working. If the two individual sources of energy share some facilities in the factory, their failure times may be dependent random variables.

In this chapter we extend the dual GMO model assuming dependence between the random variables $T_{1}$ and $T_{2}$ in (4.1). In Section 4.2 we specify the model and provide few probabilistic properties. We also obtain the corresponding copula which helps to establish aging properties of the model and the distribution of the inactivity times. Several stochastic order comparisons are presented in Section 4.3 and are illustrated with examples. Conclusions are presented on Section 4.4 .

\subsection{Dual Extended Marshall-Olkin model and probabilistic prop- erties}

Firstly we will formalize the dual extended model. After that we will establish its basic probabilistic properties, obtain the corresponding copula and illustrate its applicability with several examples.

\subsubsection{Model specification and basic probabilistic properties}

We define the dual Extended Marshall-Olkin model (to be abbreviated d-EMO hereafter) as follows. Let $T_{i}, i=1,2,3$, be continuous random variables with support in $\mathbb{R}$ satisfying the stochastic representation (4.1). Assume that the joint distribution function of $\left(T_{1}, T_{2}\right)$ is given by $F_{T_{1}, T_{2}}\left(x_{1}, x_{2}\right)=\mathbb{P}\left(T_{1} \leq x_{1}, T_{2} \leq x_{2}\right)$. Suppose that the continuous random variable $T_{3}$ is independent of $T_{1}$ and $T_{2}$. Following (4.1) we obtain the relation

$$
F_{X_{1}, X_{2}}\left(x_{1}, x_{2}\right)=\mathbb{P}\left(T_{1} \leq x_{1}, T_{2} \leq x_{2}, T_{3} \leq \min \left\{x_{1}, x_{2}\right\}\right) .
$$

Thus, we specify the d-EMO model by relation

$$
F_{X_{1}, X_{2}}\left(x_{1}, x_{2}\right)=F_{T_{1}, T_{2}}\left(x_{1}, x_{2}\right) F_{T_{3}}\left(\min \left\{x_{1}, x_{2}\right\}\right) .
$$

Observe that the dual GMO model (4.2) can be obtained from (4.3) when $T_{1}$ and $T_{2}$ are independent random variables. Picture 4.2 illustrates the relationship among the two models. 


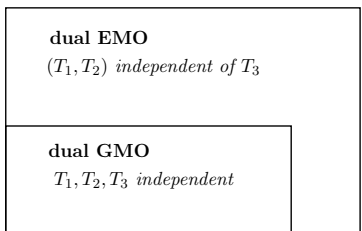

Figure 4.2: Dual GMO and dual EMO models compared.

When the random variables $T_{i}$ are absolutely continuous having density $f_{T_{i}}(x), i=1,2,3$, Barlow et al. (1963) and Keilson and Sumita (1982) define the reversed failure rate $m_{T_{i}}(x)$ and the remaining reversed failure rate $M_{T_{i}}(x)$ as

$$
m_{T_{i}}(x)=\frac{f_{T_{i}}(x)}{F_{T_{i}}(x)}=\frac{d}{d x}\left[\ln F_{T_{i}}(x)\right] \quad \text { and } \quad M_{T_{i}}(x)=\int_{x}^{\infty} m_{T_{i}}(u) \mathrm{d} u
$$

respectively. Notice that the joint distribution function admits the representation

$$
F_{T_{i}}(x)=\exp \left\{-M_{T_{i}}(x)\right\}, \quad \text { or equivalently } \quad M_{T_{i}}(x)=-\ln \left[F_{T_{i}}(x)\right], i=1,2,3 .
$$

The bivariate version of univariate exponential representation (4.4) for the joint distribution function is given by

$$
F_{T_{1}, T_{2}}\left(x_{1}, x_{2}\right)=\exp \left\{-M_{T_{1}}\left(x_{1}\right)-M_{T_{2}}\left(x_{2}\right)+A_{T_{1}, T_{2}}\left(x_{1}, x_{2}\right)\right\}
$$

where the dependence function $A_{T_{1}, T_{2}}\left(x_{1}, x_{2}\right)$ is defined by

$$
A_{T_{1}, T_{2}}\left(x_{1}, x_{2}\right)=\ln \frac{F_{T_{1}, T_{2}}\left(x_{1}, x_{2}\right)}{F_{T_{1}}\left(x_{1}\right) F_{T_{2}}\left(x_{2}\right)},
$$

see Sankaran and Gleeja (2006). Notice that the dependence function $A_{T_{1}, T_{2}}\left(x_{1}, x_{2}\right)=0$ if and only if $T_{1}$ and $T_{2}$ are independent random variables. As interpretation, the dependence function $A_{T_{1}, T_{2}}\left(x_{1}, x_{2}\right)$ postulates (describes) the association between random variables $T_{1}$ and $T_{2}$ free of (i.e. excluding) the marginal contribution into their mutual (genuine) dependence.

Under the above assumptions and notations, we define the d-EMO model as follows.

Definition 4.2.1. The joint distribution function of the d-EMO model is given by

$$
F_{X_{1}, X_{2}}\left(x_{1}, x_{2}\right)=\exp \left\{-M_{T_{1}}\left(x_{1}\right)-M_{T_{2}}\left(x_{2}\right)-M_{T_{3}}\left(\min \left(x_{1}, x_{2}\right)\right)+A_{T_{1}, T_{2}}\left(x_{1}, x_{2}\right)\right\} .
$$

Remark 4.2.2. Note that the only difference between dual GMO and d-EMO models is the presence of the dependence function $A_{T_{1}, T_{2}}\left(x_{1}, x_{2}\right)$ in (4.7), which may have important features to understand the interaction between the random variables involved.

Therefore, besides the knowledge of the distribution of $T_{3}$, a key aspect for deriving d-EMO distribution is the knowledge of joint distribution of $T_{1}$ and $T_{2}$ given by (4.5). An important component in (4.5) is the dependence function $A_{T_{1}, T_{2}}\left(x_{1}, x_{2}\right)$, given by (4.6), representing the dependence structure between $T_{1}$ and $T_{2}$ in addition to the marginal influence.

Remark 4.2.3. Let the joint distribution of the random vector $\left(T_{1}, T_{2}\right)$ be absolutely continuous having joint density $f_{T_{1}, T_{2}}\left(x_{1}, x_{2}\right)$. Bismi (2005) defined the bivariate reversed failure rate 
by $m_{T_{1}, T_{2}}\left(x_{1}, x_{2}\right)=\frac{f_{T_{1}, T_{2}}\left(x_{1}, x_{2}\right)}{F_{T_{1}, T_{2}}\left(x_{1}, x_{2}\right)}$. Denote the conditional reversed hazard rates by $m_{i}\left(x_{1}, x_{2}\right)=$ $\frac{\partial}{\partial x_{i}} \ln \left[F_{T_{1}, T_{2}}\left(x_{1}, x_{2}\right)\right], i=1,2$, see Roy (2002b). Following Sankaran and Gleeja (2006), the dependence function $A_{T_{1}, T_{2}}\left(x_{1}, x_{2}\right)$ can be written as

$$
A_{T_{1}, T_{2}}\left(x_{1}, x_{2}\right)=\int_{x_{1}}^{\infty} \int_{x_{2}}^{\infty}\left[m_{T_{1}, T_{2}}\left(y_{1}, y_{2}\right)-m_{1}\left(y_{1}, y_{2}\right) m_{2}\left(y_{1}, y_{2}\right)\right] \mathrm{d} y_{1} \mathrm{~d} y_{2} .
$$

\section{Bounds for the joint distribution function}

In the case of missing information regarding the dependence function $A_{T_{1}, T_{2}}\left(x_{1}, x_{2}\right)$, it is still possible to obtain bounds for the distribution function of the d-EMO distribution (4.7) based on knowledge of the marginal distribution functions $F_{T_{i}}(x)$ or, equivalently, in terms of their remaining reversed failure rates $M_{T_{i}}(x), i=1,2,3$. The bounds for the distribution function $F_{X_{1}, X_{2}}\left(x_{1}, x_{2}\right)$ of the d-EMO distribution are given below.

Lemma 4.2.4. The lower and upper bounds for the distribution function of the d-EMO distribution (4.7), are given by

$$
L\left(x_{1}, x_{2}\right) \leq F_{X_{1}, X_{2}}\left(x_{1}, x_{2}\right) \leq U\left(x_{1}, x_{2}\right)
$$

where

$$
L\left(x_{1}, x_{2}\right)=\max \left\{\exp \left\{-M_{T_{1}}\left(x_{1}\right)\right\}+\exp \left\{-M_{T_{2}}\left(x_{2}\right)\right\}-1,0\right\} \exp \left\{-M_{T_{3}}\left(\min \left(x_{1}, x_{2}\right)\right)\right\}
$$

and

$$
U\left(x_{1}, x_{2}\right)=\min \left\{\exp \left\{-M_{T_{1}}\left(x_{1}\right)\right\}, \exp \left\{-M_{T_{2}}\left(x_{2}\right)\right\}\right\} \exp \left\{-M_{T_{3}}\left(\min \left(x_{1}, x_{2}\right)\right)\right\} .
$$

Proof. Let a pair $\left(x_{1}, x_{2}\right)$ belong to the set $\overline{\mathbf{W}}\left(x_{1}, x_{2}\right)$ defined by

$$
\overline{\mathbf{W}}\left(x_{1}, x_{2}\right)=\left\{\left(x_{1}, x_{2}\right) \in \mathbf{R}^{2} \mid F_{T_{1}}\left(x_{1}\right)+F_{T_{2}}\left(x_{2}\right)>1\right\} .
$$

Applying the usual Fréchet-Hoeffding bounds (3.7) to the joint distribution function of $\left(T_{1}, T_{2}\right)$ written in the exponential representation (4.5), we obtain the following bounds for the dependence function $A_{T_{1}, T_{2}}\left(x_{1}, x_{2}\right)$

$$
A_{T_{1}, T_{2}}\left(x_{1}, x_{2}\right) \geq M_{T_{1}}\left(x_{1}\right)+M_{T_{2}}\left(x_{2}\right)+\ln \left[\exp \left\{-M_{T_{1}}\left(x_{1}\right)\right\}+\exp \left\{-M_{T_{2}}\left(x_{2}\right)\right\}-1\right]
$$

and

$$
A_{T_{1}, T_{2}}\left(x_{1}, x_{2}\right) \leq M_{T_{1}}\left(x_{1}\right)+M_{T_{2}}\left(x_{2}\right)+\ln \left[\min \left[\exp \left\{-M_{T_{1}}\left(x_{1}\right)\right\}, \exp \left\{-M_{T_{2}}\left(x_{2}\right)\right\}\right]\right]
$$

for all $\left(x_{1}, x_{2}\right) \in \overline{\mathbf{W}}\left(x_{1}, x_{2}\right)$. Substituting the above bounds in the expression or the joint distribution function of d-EMO models (4.7), lower and upper bounds $L\left(x_{1}, x_{2}\right)$ and $U\left(x_{1}, x_{2}\right)$ for $F_{X_{1}, X_{2}}\left(x_{1}, x_{2}\right)$ stated by Lemma 4.2 .4 will be obtained when $\left(x_{1}, x_{2}\right) \in \overline{\mathbf{W}}\left(x_{1}, x_{2}\right)$. The case when $F_{T_{1}}\left(x_{1}\right)+$ $F_{T_{2}}\left(x_{2}\right) \leq 1$ is trivial.

Note that the bounds $L\left(x_{1}, x_{2}\right)$ and $U\left(x_{1}, x_{2}\right)$ in Lemma 4.2.4 are sharper than the usual FréchetHoeffding bounds, given by (3.7), because of the multiplier $\exp \left\{-M_{T_{3}}\left(\min \left(x_{1}, x_{2}\right)\right)\right\}$. 


\section{Positive and negative quadrant dependence}

A random vector $\left(X_{1}, X_{2}\right)$ assuming values in $\mathbf{R}^{2}$ is positive quadrant dependent (PQD) if $\mathbb{P}\left(X_{1} \leq x_{1}, X_{2} \leq x_{2}\right) \geq \mathbb{P}\left(X_{1} \leq x_{1}\right) \mathbb{P}\left(X_{2} \leq x_{2}\right)$ for all $x_{1}, x_{2}$ and negative quadrant dependent (NQD) when the last relation is valid with the inequality sign reversed, see Lehmann (1966). The dual GMO model (4.2) is always PQD, see equation (3) in Fang and Li (2013). The d-EMO model (4.7) may be positively or negatively quadrant dependent, conditional on the distributional parameters involved. The next statement characterizes the NQD property of the d-EMO model.

Lemma 4.2.5. The d-EMO model (4.3) is NQD if and only if

$$
F_{T_{1}, T_{2}}\left(x_{1}, x_{2}\right) \leq F_{T_{1}}\left(x_{1}\right) F_{T_{2}}\left(x_{2}\right) F_{T_{3}}\left(\max \left\{x_{1}, x_{2}\right\}\right)
$$

for all $x_{1}, x_{2} \in \mathbf{R}$.

Proof. The necessary and sufficient condition given by relation (4.9) can be obtained from its equivalent inequality $F_{X_{1}, X_{2}}\left(x_{1}, x_{2}\right) \leq F_{X_{1}}\left(x_{1}\right) F_{X_{2}}\left(x_{2}\right)$.

The following Corollary gives another version of the inequality (4.9) in terms of dependence function $A_{T_{1}, T_{2}}\left(x_{1}, x_{2}\right)$ and the remaining reversed failure rate $M_{T_{3}}(x)$.

Corollary 4.2.6. The d-EMO model is $N Q D$ if and only if

$$
A_{T_{1}, T_{2}}\left(x_{1}, x_{2}\right)+M_{T_{3}}\left(\max \left(x_{1}, x_{2}\right)\right) \leq 0
$$

for all $x_{1}, x_{2} \in \mathbf{R}$.

Proof. Taking into account the exponential representation for d-EMO models (4.7), one gets that the marginal distribution functions have the following form in terms of remaining reversed failure rates:

$F_{X_{i}}\left(x_{i}\right)=\exp \left\{-M_{T_{i}}\left(x_{i}\right)-M_{T_{3}}\left(x_{i}\right)\right\}, i=1,2$. The vector $\left(X_{1}, X_{2}\right)$ is NQD if and only if $F_{X_{1}, X_{2}}\left(x_{1}, x_{2}\right) \leq F_{X_{1}}\left(x_{1}\right) F_{X_{2}}\left(x_{2}\right)$ for all $x_{1}, x_{2} \in \mathbf{R}$. This last inequality is equivalent to

$$
\exp \left\{A_{T_{1}, T_{2}}\left(x_{1}, x_{2}\right)+M_{T_{3}}\left(\max \left(x_{1}, x_{2}\right)\right)\right\} \leq 1,
$$

which is the inequality stated.

We will construct in the next example a d-EMO distribution which satisfies the NQD condition (4.9).

Example 4.2.7 (d-EMO distribution with NQD property). Let $T_{1}$ and $T_{2}$ be countermonotonic random variables, i.e., their joint distribution is given by the lower Fréchet bound, and define

$$
\theta=\inf \left\{x \mid(x, x) \in \overline{\mathbf{W}}\left(x_{1}, x_{2}\right)\right\}=\text { const },
$$

where the set $\overline{\mathbf{W}}\left(x_{1}, x_{2}\right)$ is defined in (4.8).

Fix the distribution of $T_{3}$ as follows: $\mathbb{P}\left(T_{3} \leq \theta\right)=1$, i.e. the support of $T_{3}$ is the interval $(-\infty, \theta]$. It is direct to check that specified distributions of $\left(T_{1}, T_{2}\right)$ and $T_{3}$ compose a d-EMO distribution which is $N Q D$. 


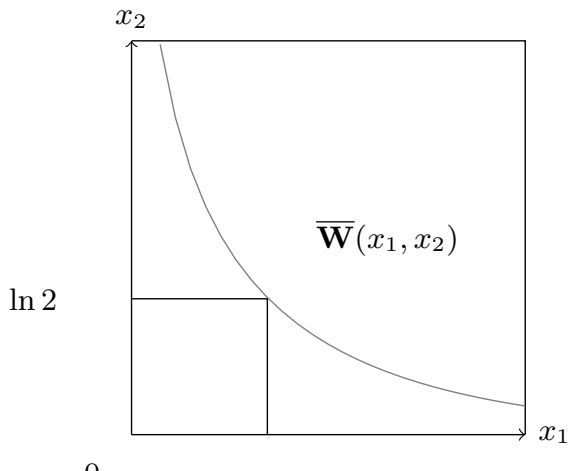

$\ln 2$

Figure 4.3: Set $\overline{\mathbf{W}}\left(x_{1}, x_{2}\right)$ in Example 4.2.\%.

For example, let us consider two unit exponential countermonotonic random variables $T_{1}$ and $T_{2}$. One may easy obtain that $\theta=\ln 2$ in this case, i.e. $\mathbb{P}\left(T_{3} \leq \ln 2\right)=1$. For the specifications considered, the set $\overline{\mathbf{W}}\left(x_{1}, x_{2}\right)$ is presented in Figure 4.3. We have

$$
F_{T_{1}, T_{2}}\left(x_{1}, x_{2}\right)<F_{T_{1}}\left(x_{1}\right) F_{T_{2}}\left(x_{2}\right) \quad \text { and } \quad F_{T_{3}}\left(\max \left(x_{1}, x_{2}\right)\right)=1
$$

if $\left(x_{1}, x_{2}\right) \in \overline{\mathbf{W}}\left(x_{1}, x_{2}\right)$, i.e. the NQD condition (4.9) holds true. Observe that $F_{T_{1}, T_{2}}\left(x_{1}, x_{2}\right)=0$ when $\left(x_{1}, x_{2}\right) \notin \overline{\mathbf{W}}\left(x_{1}, x_{2}\right)$. Therefore, the corresponding vector $\left(X_{1}, X_{2}\right)$ defined by (4.3) possesses the NQD property.

Remark 4.2.8. It would be optimistic to expect in a real situation that $N Q D$ property of the vector $\left(X_{1}, X_{2}\right)$ would be valid for all observed pairs $\left(x_{1}^{*}, x_{2}^{*}\right)$ which should belong to the subset $\overline{\mathbf{W}}\left(x_{1}, x_{2}\right)$ defined in (4.8). In such cases, it is convenient to interpret "locally" the NQD property of $\left(X_{1}, X_{2}\right)$ when some pairs of observations are outside the set overline $\mathbf{W}\left(x_{1}, x_{2}\right)$, but the practical situation clearly indicates the use of NQD model. Example 4.2.16 supports this conclusion in copula terms.

Remark 4.2.9. Recall that if a bivariate distribution is $P Q D$, then for every pair of increasing function $g_{i}(x), i=1,2$, we have $\operatorname{Cov}\left\{g_{1}\left(X_{1}\right), g_{2}\left(X_{2}\right)\right\} \geq 0$, see Barlow and Proschan (1981). Such a restriction holds for dual GMO distributions, see Fang and Li (2013), but does not apply to d-EMO distributions.

\section{Symmetry and asymmetry}

The dual GMO model (4.1) may be exchangeable or not, depending on the distributions that $T_{1}$ and $T_{2}$ follow: if identically distributed then the dual GMO model is symmetric. Interestingly, in the d-EMO model (4.3), one may start with identically distributed random variables $T_{1}$ and $T_{2}$, but connected by a non exchangeable copula. Then the resulting d-EMO distribution will be non exchangeable. In fact, we have the next Lemma.

Lemma 4.2.10. The d-EMO model is not necessarily exchangeable. 


\subsubsection{Copula of the d-EMO distribution}

By Sklar's theorem, the dependence structure of a continuous bivariate random vector $\left(X_{1}, X_{2}\right)$ can be described by unique copula

$$
C_{X_{1}, X_{2}}(u, v)=F_{X_{1}, X_{2}}\left(F_{X_{1}}^{-1}(u), F_{X_{2}}^{-1}(v)\right), \quad(u, v) \in[0,1]
$$

where $F_{X_{i}}^{-1}(u)$ is the (generalized) inverse of $F_{X_{i}}(x), i=1,2$, see Theorem 2.2.3 in Nelsen (2006).

As we noted in the proof of the Corollary 4.2.6, the marginal distribution functions of $F_{X_{1}, X_{2}}\left(x_{1}, x_{2}\right)$ from (4.7) are given by $F_{X_{i}}\left(x_{i}\right)=\exp \left\{-M_{T_{i}}\left(x_{i}\right)-M_{T_{3}}\left(x_{i}\right)\right\}$. Denote by $\bar{G}_{i}\left(x_{i}\right)=M_{T_{i}}\left(x_{i}\right)+$ $M_{T_{3}}\left(x_{i}\right), i=1,2$. Under the above relations, the following statement is true.

Lemma 4.2.11. The copula $C_{X_{1}, X_{2}}(u, v)$ of the d-EMO distribution is given by

$$
C_{X_{1}, X_{2}}(u, v)=\left\{\begin{array}{lll}
u v \exp \left\{M_{T_{3}}\left(\bar{G}_{1}^{-1}(-\ln u)\right)+\bar{G}(u, v)\right\}, & \text { if } \quad \bar{G}_{1}^{-1}(-\ln u)>\bar{G}_{2}^{-1}(-\ln v) ; \\
u v \exp \left\{M_{T_{3}}\left(\bar{G}_{2}^{-1}(-\ln v)\right)+\bar{G}(u, v)\right\}, & \text { if } \quad \bar{G}_{1}^{-1}(-\ln u) \leq \bar{G}_{2}^{-1}(-\ln v),
\end{array}\right.
$$

where $u, v \in(0,1]$ and $\bar{G}(u, v)=A_{T_{1}, T_{2}}\left(F_{X_{1}}^{-1}(u), F_{X_{2}}^{-1}(v)\right)$.

Proof. Let $C_{X_{1}, X_{2}}(u, v)$ be the copula corresponding to $F_{X_{1}, X_{2}}\left(x_{1}, x_{2}\right)$ given by (4.7), i.e.

$$
\ln \left[C_{X_{1}, X_{2}}(u, v)\right]=\ln \left[F_{X_{1}, X_{2}}\left(F_{X_{1}}^{-1}(u), F_{X_{2}}^{-1}(v)\right)\right], \quad u, v \in(0,1]
$$

Because of continuity of the distributions involved we have unique inverses.

Since $F_{X_{1}}\left(x_{1}\right)=\exp \left\{-M_{T_{1}}\left(x_{1}\right)-M_{T_{3}}\left(x_{1}\right)\right\}=\exp \left\{-\bar{G}_{1}\left(x_{1}\right)\right\}$, we have

$$
F_{X_{1}}^{-1}(u)=\bar{G}_{1}^{-1}(-\ln u), \quad \text { implying } \quad \bar{G}_{1}\left(F_{X_{1}}^{-1}(u)\right)=-\ln u \text {. }
$$

In a similar way, from $F_{X_{2}}\left(x_{2}\right)=\exp \left\{-M_{T_{2}}\left(x_{2}\right)-M_{T_{3}}\left(x_{2}\right)\right\}=\exp \left\{-\bar{G}_{2}\left(x_{2}\right)\right\}$ we obtain

$$
F_{X_{2}}^{-1}(v)=\bar{G}_{2}^{-1}(-\ln v), \quad \text { i.e. } \quad \bar{G}_{2}\left(F_{X_{2}}^{-1}(v)\right)=-\ln v .
$$

Let $x_{1}>x_{2}$, i.e. $F_{X_{1}}^{-1}(u)>F_{X_{2}}^{-1}(v)$, or equivalently $\bar{G}_{1}^{-1}(-\ln u)>\bar{G}_{2}^{-1}(-\ln v)$. Using the exponential representation for d-EMO distributions (4.7) we have

$$
\ln \left[C_{X_{1}, X_{2}}(u, v)\right]=\ln \left\{\exp \left\{-M_{T_{1}}\left(F_{X_{1}}^{-1}(u)\right)-M_{T_{2}}\left(F_{X_{2}}^{-1}(v)\right)-M_{T_{3}}\left(F_{X_{2}}^{-1}(v)\right)+\bar{G}(u, v)\right\}\right\},
$$

with $\bar{G}(u, v)=A_{T_{1}, T_{2}}\left(F_{X_{1}}^{-1}(u), F_{X_{2}}^{-1}(v)\right)$, which is equivalent to

$$
\ln \left[C_{X_{1}, X_{2}}(u, v)\right]=-M_{T_{1}}\left(F_{X_{1}}^{-1}(u)\right)+\ln v+\bar{G}(u, v) .
$$

Due to (4.11) we get

$$
\ln C_{X_{1}, X_{2}}(u, v)=\ln u v+M_{T_{3}}\left(F_{X_{1}}^{-1}(u)\right)+\bar{G}(u, v) .
$$

Finally, $C_{X_{1}, X_{2}}(u, v)=u v \exp \left\{M_{T_{3}}\left(\bar{G}_{1}^{-1}(-\ln u)\right)+\bar{G}(u, v)\right\}$ if $F_{X_{1}}^{-1}(u)>F_{X_{2}}^{-1}(v)$. 
By analogy, when $x_{1} \leq x_{2}$, i.e. $\bar{G}_{1}^{-1}(-\ln u) \leq \bar{G}_{2}^{-1}(-\ln v)$, one obtains

$$
C_{X_{1}, X_{2}}(u, v)=u v \exp \left\{M_{T_{3}}\left(\bar{G}_{2}^{-1}(-\ln v)\right)+\bar{G}(u, v)\right\}
$$

and representation (4.10) is established.

Remark 4.2.12. The function $\exp \{\bar{G}(u, v)\}$ is the only product extra multiplier in (4.10) in addition to the copula expression corresponding to dual GMO distribution and numbered (4) by Fang and Li (2013). This extra term permits $N Q D$ modelling on $\left(X_{1}, X_{2}\right)$.

Remark 4.2.13. Whenever $\left(T_{1}, T_{2}\right)$ is absolutely continuous, using Remark 4.2.3, we have the alternative representation for $\bar{G}(u, v)=A_{T_{1}, T_{2}}\left(F_{X_{1}}^{-1}(u), F_{X_{2}}^{-1}(v)\right)$,

$$
\bar{G}(u, v)=\int_{F_{X_{1}}^{-1}(u)}^{\infty} \int_{F_{X_{2}}^{-1}(v)}^{\infty}\left[m_{T_{1}, T_{2}}\left(y_{1}, y_{2}\right)-m_{1}\left(y_{1}, y_{2}\right) m_{2}\left(y_{1}, y_{2}\right)\right] \mathrm{d} y_{1} \mathrm{~d} y_{2} .
$$

The following two examples illustrate how to get the copula representation (4.10) of d-EMO distribution in particular cases.

Example 4.2.14 (Copula of d-EMO distribution under proportional reversed failure rate marginals). Consider a baseline remaining reversed failure rate function $M(x)$ and suppose $F_{T_{i}}(x)=[\exp \{-M(x)\}]^{\lambda_{i}}$, $\lambda_{i}>0, i=1,2,3$, see Gupta et al. (1998). After some algebra relation (4.10) simplifies to

$$
C_{X_{1}, X_{2}}(u, v)=\left\{\begin{array}{lll}
u^{\frac{\lambda_{1}}{\lambda_{1}+\lambda_{3}}} v \exp \{\bar{G}(u, v)\}, & \text { if } \quad \bar{G}_{1}^{-1}(-\ln u)>\bar{G}_{2}^{-1}(-\ln v) \\
u v^{\frac{\lambda_{2}}{\lambda_{2}+\lambda_{3}}} \exp \{\bar{G}(u, v)\}, & \text { if } \quad \bar{G}_{1}^{-1}(-\ln u) \leq \bar{G}_{2}^{-1}(-\ln v)
\end{array}\right.
$$

where $u, v \in(0,1]$. Notice that when $T_{1}$ and $T_{2}$ are independent we have $\bar{G}(u, v)=0$ for all $u, v \in$ $(0,1]$ and we obtain the survival copula of the Marshall-Olkin bivariate exponential distribution given by (2.15), see the corresponding comments in Li (2008).

Example 4.2.15 (Copula of the d-EMO distribution given the marginal distributions and internal dependence structure). Let $T_{1}$ and $T_{2}$ follow Fréchet distribution with parameter $\alpha>0$, i.e. $F_{T_{i}}\left(x_{i}\right)=\exp \left\{-x_{i}^{-\alpha}\right\}$ for $x_{i}>0, i=1,2$. Assume that the vector $\left(T_{1}, T_{2}\right)$ is Farlie-GumbelMorgenstern distributed, e.g.

$$
F_{T_{1}, T_{2}}\left(x_{1}, x_{2}\right)=\exp \left\{-x_{1}^{-\alpha}-x_{2}^{-\alpha}\right\}\left[1+\theta\left(1-\exp \left\{-x_{1}^{-\alpha}\right\}\right)\left(1-\exp \left\{-x_{2}^{-\alpha}\right\}\right)\right]
$$

where $\theta \in[-1,1]$, see Family B10 in Joe (1997). From relation (4.6) we obtain the dependence function

$$
A_{T_{1}, T_{2}}\left(x_{1}, x_{2}\right)=\ln \frac{F_{T_{1}, T_{2}}\left(x_{1}, x_{2}\right)}{F_{T_{1}}\left(x_{1}\right) F_{T_{2}}\left(x_{2}\right)}=\ln \left[1+\theta\left(1-\exp \left\{-x_{1}^{-\alpha}\right\}\right)\left(1-\exp \left\{-x_{2}^{-\alpha}\right\}\right)\right] .
$$

Fix $T_{3}$ to be independent of $\left(T_{1}, T_{2}\right)$ with distribution $F_{T_{3}}(x)=\exp \left\{-x^{-2 \alpha}\right\}, x>0$. Following the above specifications, we have $M_{T_{1}}(x)=M_{T_{2}}(x)=x^{-\alpha}$, and $M_{T_{3}}(x)=x^{-2 \alpha}$. Then $\bar{G}_{i}(x)=$ 
$M_{T_{i}}(x)+M_{T_{3}}(x)=x^{-\alpha}+x^{-2 \alpha}$ and therefore

$$
\bar{G}_{i}^{-1}(u)=\left[\sqrt[\alpha]{\frac{-1+\sqrt{1+4 u}}{2}}\right]^{-1} \quad \text { and } \quad F_{X_{i}}^{-1}(u)=\left[\sqrt[\alpha]{\frac{-1+\sqrt{1-4 \ln u}}{2}}\right]^{-1}, i=1,2 .
$$

Applying $\bar{G}(u, v)=A_{T_{1}, T_{2}}\left(F_{X_{1}}^{-1}(u), F_{X_{2}}^{-1}(v)\right)$ we have

$$
\bar{G}(u, v)=\ln \left[1+\theta\left(1-\exp \left\{-\frac{-1+\sqrt{1-4 \ln u}}{2}\right\}\right)\left(1-\exp \left\{-\frac{-1+\sqrt{1-4 \ln v}}{2}\right\}\right)\right] .
$$

Substituting above relations in the expression for the copula of d-EMO distribution (4.10) we get

$$
C_{X_{1}, X_{2}}(u, v)=\left\{\begin{array}{l}
u v \exp \left\{\frac{-1+\sqrt{1-4 \ln u}}{2}\right\}^{2} \\
\times\left[1+\theta\left(1-\exp \left\{-\frac{-1+\sqrt{1-4 \ln u}}{2}\right\}\right)\left(1-\exp \left\{-\frac{-1+\sqrt{1-4 \ln v}}{2}\right\}\right)\right], \quad \text { if } \quad u>v ; \\
u v \exp \left\{\frac{-1+\sqrt{1-4 \ln v}}{2}\right\}^{2} \\
\times\left[1+\theta\left(1-\exp \left\{-\frac{-1+\sqrt{1-4 \ln u}}{2}\right\}\right)\left(1-\exp \left\{-\frac{-1+\sqrt{1-4 \ln v}}{2}\right\}\right)\right], \quad \text { if } \quad u \leq v ;
\end{array}\right.
$$

where $u, v \in(0,1]$.

In the next example we use the copula from Example 4.2.15 to justify the comments in Remark 4.2 .8 , i.e. the same d-EMO copula may be "locally" PQD and NQD.

Example 4.2.16 (Quadrant dependence analysis of the copula from Example 4.2.15). Consider the d-EMO copula of Example 4.2.15. Whenever $\theta \in[-1,0]$ the joint distribution of $\left(T_{1}, T_{2}\right)$ is $N Q D$, as a property of the Farlie-Gumbel-Morgenstern copula. Let us consider the extremal negative dependence, substituting $\theta=-1$, and define the decreasing function

$$
B(u)=\frac{-1+\sqrt{1-4 \ln u}}{2}
$$

When $1 \geq u>v>0, C_{\left.X_{1}, X_{2}\right)}(u, v)$ is $N Q D$ if and only if

$$
\exp \{-B(u)\}+\exp \{-B(v)\}[1-\exp \{-B(u)\}] \leq \exp \left\{-B^{2}(u)\right\},
$$

which is equivalent to

$$
[1-\exp \{-B(u)\}][1-\exp \{-B(v)\}] \geq 1-\exp \left\{-B^{2}(u)\right\} .
$$

Since the function $1-\exp \{-B(v)\}$ is decreasing and $1-\exp \{-B(0+)\}=1$, the former condition holds true if and only if

$$
0 \leq B(u) \leq 1 \text { and } \exp \{-B(v)\} \leq \frac{\exp \left\{-B^{2}(u)\right\}-\exp \{-B(u)\}}{1-\exp \{-B(u)\}} .
$$

After some algebra, one can conclude that the local NQD property holds if and only if $(u, v) \in N_{u>v}$ where

$$
N_{u>v}=\left\{(u, v) \in(0,1]^{2} \mid \exp \{-2\} \leq u \leq 1 \text { and } 0 \leq v \leq \exp \left\{\frac{1-\left(1-2 \ln \frac{\exp \left\{-B^{2}(u)\right\}-\exp \{-B(u)\}}{1-\exp \{-B(u)\}}\right)^{2}}{4}\right\}\right\} .
$$




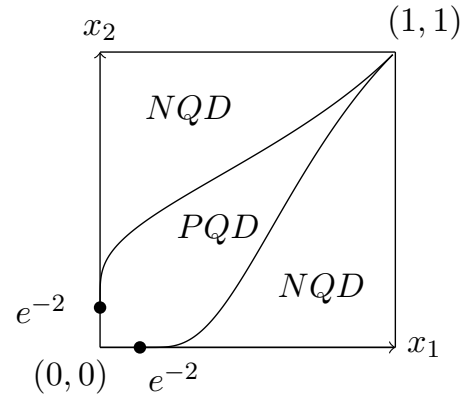

Figure 4.4: Dependence analysis of the d-EMO distribution in Example 4.2.16

By analogy, when $0<u \leq v \leq 1, C_{X_{1}, X_{2}}(u, v)$ is locally $N Q D$ if and only if $(u, v) \in N_{v \geq u}$ where

$$
N_{v \geq u}=\left\{(u, v) \in(0,1]^{2} \mid \exp \{-2\} \leq v \leq 1 \text { and } 0 \leq u \leq \exp \left\{\frac{1-\left(1-2 \ln \frac{\exp \left\{-B^{2}(v)\right\}-\exp \{-B(v)\}}{1-\exp \{-B(v)\}}\right)^{2}}{4}\right\}\right\} .
$$

Therefore, for $(u, v) \in(0,1]^{2}$, whenever $(u, v) \in\left\{N_{u>v} \cup N_{v \geq u}\right\}$ we have the local NQD property and outside this set, the local $P Q D$ property, see Figure 4.4.

\subsubsection{Preservation of the d-EMO distribution}

Let $\mathbf{X}=\left(X_{1}, X_{2}\right)$ be a bivariate random vector following the d-EMO model (4.7). Denote by

$$
\mathbf{X}_{(t)}=\left[\left(t-X_{1}, t-X_{2}\right) \mid X_{1} \leq t, X_{2} \leq t\right], \quad t \geq 0
$$

the corresponding inactivity times vector which has nonnegative marginals. Consider the vector

$$
-\mathbf{X}_{(t)}=\left[\left(X_{1}-t, X_{2}-t\right) \mid X_{1} \leq t, X_{2} \leq t\right]
$$

which is the symmetric image of $\mathbf{X}_{(t)}$ with respect to the point $(0,0)$. We are interested to examine the distribution of the random vector $-\mathbf{X}_{(t)}$. The result is given in the following

Lemma 4.2.17. If the vector $\mathbf{X}=\left(X_{1}, X_{2}\right)$ follows a d-EMO distribution, then so does $-\mathbf{X}_{(t)}$.

Proof. Since the random vector in (4.13) is non positive, consider $y_{1} \leq 0$ and $y_{2} \leq 0$. Because of the stochastic representation (4.1), the joint distribution of $-\mathbf{X}_{(t)}$ is given by

$$
\mathbb{P}\left(\max \left\{T_{1}, T_{3}\right\}-t \leq y_{1}, \max \left\{T_{2}, T_{3}\right\}-t \leq y_{2} \mid \max \left\{T_{1}, T_{3}\right\} \leq t, \max \left\{T_{2}, T_{3}\right\} \leq t\right) .
$$

Taking into account that in the d-EMO model the random variable $T_{3}$ is independent of the vector $\left(T_{1}, T_{2}\right)$ the last probability can be rewritten as

$$
\mathbb{P}\left(T_{1}-t \leq y_{1}, T_{2}-t \leq y_{2} \mid T_{1} \leq t, T_{2} \leq t\right) \mathbb{P}\left(T_{3}-t \leq \min \left\{y_{1}, y_{2}\right\} \mid T_{3} \leq t\right) .
$$

The last relation means that

$$
\mathbb{P}\left(-\mathbf{X}_{(t)} \leq\left(y_{1}, y_{2}\right)\right)=\mathbb{P}\left(-\mathbf{T}_{12(t)} \leq\left(y_{1}, y_{2}\right)\right) \mathbb{P}\left(-T_{3(t)} \leq \min \left\{y_{1}, y_{2}\right\}\right)
$$

where $-\mathbf{T}_{12(t)}=\left[\left(T_{1}-t, T_{2}-t\right) \mid T_{1} \leq t, T_{2} \leq t\right]$ and $-T_{3(t)}=\left[T_{3}-t \mid T_{3} \leq t\right]$. Therefore, for any 
$t>0$, the vector $-\mathbf{X}_{(t)}$ admits the stochastic representation

$$
-\mathbf{X}_{(t)}=\left[\max \left\{-T_{1(t)},-T_{3(t)}\right\}, \max \left\{-T_{2(t)},-T_{3(t)}\right\}\right],
$$

where $-T_{1(t)}$ and $-T_{2(t)}$ are the marginals of $-\mathbf{T}_{12(t)}$. Thus, $-\mathbf{X}_{(t)}$ satisfies the stochastic representation (4.1). This means that if the random vector $\left(X_{1}, X_{2}\right)$ is d-EMO distributed, then so does $-\mathbf{X}_{(t)}$.

Corollary 4.2.18. If the vector $\mathbf{X}=\left(X_{1}, X_{2}\right)$ follows a d-EMO distribution, then $\mathbf{X}$ and $-\mathbf{X}_{(t)}$ have the same type of copula, given by relation (4.10).

Remark 4.2.19. Similar result is valid for the Extended MO model defined by the random vector

$$
\left(Y_{1}, Y_{2}\right)=\left[\min \left(Z_{1}, Z_{3}\right), \min \left(Z_{2}, Z_{3}\right)\right]
$$

where the nonnegative random variables $Z_{1}$ and $Z_{2}$ are dependent, but being independent of $Z_{3}$. In this case, the residual lifetime vector

$$
\mathbf{X}_{t}=\left[\left(X_{1}-t, X_{2}-t\right) \mid X_{1}>t, X_{2}>t\right], \quad t \geq 0
$$

follows EMO model, see Theorem 3.3.6.

\subsection{Stochastic order comparisons}

In this section we will present several stochastic order comparisons between bivariate random vectors that follow d-EMO distributions. The first result is related to the usual stochastic order between random vectors $\left(X_{1}, X_{2}\right)$ and $\left(Y_{1}, Y_{2}\right)$, to be denoted by $\left(X_{1}, X_{2}\right) \leq_{s t}\left(Y_{1}, Y_{2}\right)$. To remind, this means that $\mathrm{E}\left[\psi\left(X_{1}, X_{2}\right)\right] \leq \mathrm{E}\left[\psi\left(Y_{1}, Y_{2}\right)\right]$ for every increasing function $\psi$ such that expectation exists, see Shaked and Shanthikumar (2007).

Lemma 4.3.1. Consider continuous random variables $S_{i}$ and $T_{i}, i=1,2,3$, and suppose $\left(S_{1}, S_{2}\right) \leq_{s t}$ $\left(T_{1}, T_{2}\right)$ and $S_{3} \leq_{s t} T_{3}$. Then $\left(X_{1}, X_{2}\right) \leq_{s t}\left(Y_{1}, Y_{2}\right)$, where

$$
\left(X_{1}, X_{2}\right)=\left[\max \left(S_{1}, S_{3}\right), \max \left(S_{2}, S_{3}\right)\right] \text { and }\left(Y_{1}, Y_{2}\right)=\left[\max \left(T_{1}, T_{3}\right), \max \left(T_{2}, T_{3}\right)\right]
$$

follows a d-EMO distribution.

Proof. Since $S_{3}$ is independent of $\left(S_{1}, S_{2}\right)$ and $T_{3}$ is independent of $\left(T_{1}, T_{2}\right)$, we have $\left(S_{1}, S_{2}, S_{3}\right) \leq_{s t}\left(T_{1}, T_{2}, T_{3}\right)$. Taking into account that $\max (x, y)$ is increasing in $x$ and $y$, the result follows from Theorem 6.B.16(a) from Shaked and Shanthikumar (2007).

Remark 4.3.2. Notice that the function $g\left(s_{1}, s_{2}, s_{3}\right)=\left[\max \left(s_{1}, s_{3}\right), \max \left(s_{2}, s_{3}\right)\right]$ is increasing and convex in its arguments. Thus, Lemma 4.3.1 remains valid if we replace the usual stochastic order by the increasing convex order, as a direct application of Theorem 7.A.5(a) from Shaked and Shanthikumar (2007).

The concordance order for copulas of d-EMO distributions is considered in the sequel. First will we need Lemma 4.3.3, which is proved in Fang and Li (2013), see their Theorem 2. 
Lemma 4.3.3 (Fang and Li (2013)). Suppose $S_{i}$ and $T_{i}, i=1,2,3$, are independent and continuous random variables such that $S_{i} \leq_{s t} T_{i}, i=1,2$, and $S_{3} \geq_{s t} T_{3}$. Then $C_{X_{1}, X_{2}}(u, v) \geq C_{Y_{1}, Y_{2}}(u, v)$ for all $u, v \in[0,1]$ where

$$
\left(X_{1}, X_{2}\right)=\left[\max \left(S_{1}, S_{3}\right), \max \left(S_{2}, S_{3}\right)\right] \text { and }\left(Y_{1}, Y_{2}\right)=\left[\max \left(T_{1}, T_{3}\right), \max \left(T_{2}, T_{3}\right)\right] .
$$

When the individual shocks in the d-EMO model are no more independent, it is still possible to obtain similar results, but additional assumptions concerning the dependence structure have to be stated.

In Lemma 4.3.4 we compare the copulas of d-EMO distributions obtained from the same pair of random variables $\left(S_{1}, S_{2}\right)$ but considering two different common shocks represented by random variables $S_{3}$ and $T$.

Lemma 4.3.4. Suppose $S_{i}, i=1,2,3$, and $T$ are continuous random variables and $A_{S_{1}, S_{2}}\left(x_{1}, x_{2}\right)=$ $\ln \frac{F_{S_{1}, S_{2}}\left(x_{1}, x_{2}\right)}{F_{S_{1}}\left(x_{1}\right) F_{S_{2}}\left(x_{2}\right)}$ is non-decreasing in $x_{1}$ and $x_{2}$. If $S_{3} \geq_{s t} T$, then $C_{X_{1}, X_{2}}(u, v) \geq C_{Y_{1}, Y_{2}}(u, v)$ for all $u, v \in[0,1]$ where

$$
\left(X_{1}, X_{2}\right)=\left[\max \left(S_{1}, S_{3}\right), \max \left(S_{2}, S_{3}\right)\right] \text { and }\left(Y_{1}, Y_{2}\right)=\left[\max \left(S_{1}, T\right), \max \left(S_{2}, T\right)\right]
$$

follow d-EMO distributions.

Proof. Since $F_{X_{i}}(x)=F_{S_{i}}(x) F_{S_{3}}(x), F_{Y_{i}}(x)=F_{S_{i}}(x) F_{T}(x)$ and $S_{3} \geq_{s t} T$, then $F_{X_{i}}^{-1}(u) \geq F_{Y_{i}}^{-1}(u)$ $i=1,2$. Thus, $A_{S_{1}, S_{2}}\left(F_{X_{1}}^{-1}(u), F_{X_{2}}^{-1}(v)\right) \geq A_{S_{1}, S_{2}}\left(F_{Y_{1}}^{-1}(u), F_{Y_{2}}^{-1}(v)\right)$. The result follows from the expression for the copula of d-EMO distributions (4.10) and Lemma 4.3.3.

Example 4.3.5 (Copula comparison for the same dependence function $A_{S_{1}, S_{2}}\left(x_{1}, x_{2}\right)$ ). Consider the Ali-Mikhail-Haq copula, see family (4.2.3) in Nelsen (2006), and suppose $\left(S_{1}, S_{2}\right)$ is jointly distributed as

$$
F_{S_{1}, S_{2}}\left(x_{1}, x_{2}\right)=\frac{\left(1-\exp \left\{-\lambda_{1} x_{1}\right\}\right)\left(1-\exp \left\{-\lambda_{2} x_{2}\right\}\right)}{1-\theta \exp \left\{-\lambda_{1} x_{1}-\lambda_{2} x_{2}\right\}},
$$

with $\theta \in[-1,0], x_{i} \geq 0$ and $\lambda_{i}>0, i=1,2$.

Therefore, $A_{S_{1}, S_{2}}\left(x_{1}, x_{2}\right)=\ln \left[\frac{1}{1-\theta \exp \left\{-\lambda_{1} x_{1}-\lambda_{2} x_{2}\right\}}\right]$ is non-decreasing in $x_{1}$ and $x_{2}$, as required by Lemma 4.3.4.

Fix $F_{S_{3}}(x)=1-\exp \left\{-\lambda_{3} x\right\}$ and $F_{T}(x)=1-\exp \left\{-\lambda_{4} x\right\}$, where $x \geq 0, \lambda_{4} \geq \lambda_{3}>0$. Hence $S_{3} \geq_{s t} T$, which implies $F_{S_{3}}^{-1}(u) \geq F_{T}^{-1}(u)$ and

$$
F_{X_{i}}(x)=F_{S_{i}}(x) F_{S_{3}}(x) \leq F_{S_{i}}(x) F_{T}(x)=F_{Y_{i}}(x) \text {, i.e. } F_{X_{i}}^{-1}(u) \geq F_{Y_{i}}^{-1}(u), i=1,2 .
$$

Notice that

$$
\begin{aligned}
F_{X_{i}}\left(F_{S_{3}}^{-1}(u)\right) & =F_{S_{i}}\left(F_{S_{3}}^{-1}(u)\right) F_{S_{3}}\left(F_{S_{3}}^{-1}(u)\right)=F_{S_{i}}\left(F_{S_{3}}^{-1}(u)\right) u=F_{S_{i}}\left(F_{S_{3}}^{-1}(u)\right) F_{T}\left(F_{T}^{-1}(u)\right) \\
& \geq F_{S_{i}}\left(F_{T}^{-1}(u)\right) F_{T}\left(F_{T}^{-1}(u)\right)=F_{Y_{i}}\left(F_{T}^{-1}(u)\right),
\end{aligned}
$$

i.e., $F_{S_{3}}\left(F_{X_{i}}^{-1}(u)\right) \leq F_{T}\left(F_{Y_{i}}^{-1}(u)\right), i=1,2$. Thus, from the expression for the copula of $d$-EMO 
distributions (4.10) we have

$$
\begin{aligned}
C_{X_{1}, X_{2}}(u, v) & =u v \min \left\{\frac{1}{F_{S_{3}}\left(F_{X_{1}}^{-1}(u)\right)}, \frac{1}{F_{S_{3}}\left(F_{X_{2}}^{-1}(v)\right)}\right\} \exp \left\{G\left(F_{X_{1}}^{-1}(u), F_{X_{2}}^{-1}(v)\right)\right\} \\
& \geq u v \min \left\{\frac{1}{F_{T}\left(F_{Y_{1}}^{-1}(u)\right)}, \frac{1}{F_{T}\left(F_{Y_{2}}^{-1}(v)\right)}\right\} \exp \left\{G\left(F_{X_{1}}^{-1}(u), F_{X_{2}}^{-1}(v)\right)\right\} \\
& \geq u v \min \left\{\frac{1}{F_{T}\left(F_{Y_{1}}^{-1}(u)\right)}, \frac{1}{F_{T}\left(F_{Y_{2}}^{-1}(v)\right)}\right\} \exp \left\{G\left(F_{Y_{1}}^{-1}(u), F_{Y_{2}}^{-1}(v)\right)\right\} \\
& =C_{Y_{1}, Y_{2}}(u, v) .
\end{aligned}
$$

In Lemma 4.3.6 we consider the case when two d-EMO distributions are composed by the same common shock (represented by a random variable $S_{3}$ ) and different individual shocks, represented by the pairs of random variables $\left(S_{1}, S_{2}\right)$ and $\left(T_{1}, T_{2}\right)$.

Lemma 4.3.6. Suppose $S_{i}, i=1,2,3$, and $T_{i}, i=1,2$, are continuous random variables and let $A_{S_{1}, S_{2}}\left(x_{1}, x_{2}\right)=\ln \frac{F_{S_{1}, S_{2}}\left(x_{1}, x_{2}\right)}{F_{S_{1}}\left(x_{1}\right) F_{S_{2}}\left(x_{2}\right)} \geq A_{T_{1}, T_{2}}\left(y_{1}, y_{2}\right)=\ln \frac{F_{T_{1}, T_{2}}\left(y_{1}, y_{2}\right)}{F_{T_{1}}\left(y_{1}\right) F_{T_{2}}\left(y_{2}\right)}$ for $x_{i} \leq y_{i}, i=1,2$. Define the d-EMO distributions

$$
\left(X_{1}, X_{2}\right)=\left[\max \left(S_{1}, S_{3}\right), \max \left(S_{2}, S_{3}\right)\right] \text { and }\left(Y_{1}, Y_{2}\right)=\left[\max \left(T_{1}, S_{3}\right), \max \left(T_{2}, S_{3}\right)\right]
$$

If $S_{1} \leq_{s t} T_{1}, S_{2} \leq_{s t} T_{2}$, then $C_{X_{1}, X_{2}}(u, v) \geq C_{Y_{1}, Y_{2}}(u, v)$ for all $u, v \in[0,1]$.

Proof. From the stochastic ordering among the random variables involved, we have $F_{X_{i}}^{-1}(u) \leq F_{Y_{i}}^{-1}(u), i=1,2$. Since $A_{S_{1}, S_{2}}\left(F_{X_{1}}^{-1}(u), F_{X_{2}}^{-1}(v)\right) \geq A_{T_{1}, T_{2}}\left(F_{Y_{1}}^{-1}(u), F_{Y_{2}}^{-1}(v)\right)$, the result follows from the expression for the copula of d-EMO distributions (4.10) and Lemma 4.3.3.

Corollary 4.3.7. Under the assumptions of Lemma 4.3.6 we have $\left(X_{1}, X_{2}\right) \leq_{s t}\left(Y_{1}, Y_{2}\right)$.

Proof. From the exponential representation for bivariate distribution functions $(4.5)$ we have $\left(S_{1}, S_{2}\right) \leq_{s t}$ $\left(T_{1}, T_{2}\right)$ and the result follows as a direct application of Lemma 4.3.1.

Example 4.3.8 (Copula comparison). Suppose $\left(S_{1}, S_{2}\right)$ and $\left(T_{1}, T_{2}\right)$ are jointly distributed as

$$
F_{S_{1}, S_{2}}\left(x_{1}, x_{2}\right)=\frac{\left(1-\exp \left\{-\lambda_{1} x_{1}\right\}\right)\left(1-\exp \left\{-\lambda_{1} x_{2}\right\}\right)}{1-\theta_{1} \exp \left\{-\lambda_{1} x_{1}-\lambda_{1} x_{2}\right\}}, \quad \theta_{1} \in[0,1]
$$

and

$$
F_{T_{1}, T_{2}}\left(x_{1}, x_{2}\right)=\frac{\left(1-\exp \left\{-\lambda_{2} x_{1}\right\}\right)\left(1-\exp \left\{-\lambda_{2} x_{2}\right\}\right)}{1-\theta_{2} \exp \left\{-\lambda_{2} x_{1}-\lambda_{2} x_{2}\right\}}, \quad \theta_{2} \in[-1,0],
$$

where $\lambda_{1} \geq \lambda_{2}>0$ and $x_{i} \geq 0, i=1,2$.

Therefore, for all $x_{1} \leq y_{1}$ and $x_{2} \leq y_{2}$ we have

$$
\begin{aligned}
A_{S_{1}, S_{2}}\left(x_{1}, x_{2}\right) & =\ln \left[\frac{1}{1-\theta_{1} \exp \left\{-\lambda_{1} x_{1}-\lambda_{1} x_{2}\right\}}\right] \\
& \geq \ln \left[\frac{1}{1-\theta_{2} \exp \left\{-\lambda_{2} y_{1}-\lambda_{2} y_{2}\right\}}\right]=A_{T_{1}, T_{2}}\left(y_{1}, y_{2}\right) .
\end{aligned}
$$

Since $S_{i} \leq_{s t} T_{i}, i=1,2$ from Lemma 4.3.6 we have $C_{X_{1}, X_{2}}(u, v) \geq C_{Y_{1}, Y_{2}}(u, v)$ for all $u, v \in(0,1]$ 
where

$$
\left(X_{1}, X_{2}\right)=\left(\max \left(S_{1}, S_{3}\right), \max \left(S_{2}, S_{3}\right)\right) \text { and }\left(Y_{1}, Y_{2}\right)=\left(\max \left(T_{1}, S_{3}\right), \max \left(T_{2}, S_{3}\right)\right) .
$$

Notice that the copula that joins $S_{1}$ and $S_{2}$ has the $P Q D$ property while the corresponding copula of $T_{1}$ and $T_{2}$ is NQD. In addition, one may choose arbitrary continuous distribution for $S_{3}$.

Example 4.3.9 (Insurance application). Consider an insurance company offering two policies. The first policy covers a pair of random losses $\left(X_{1}, X_{2}\right)$ which are subject to dependent individual risks (with potential losses $S_{1}$ and $S_{2}$ ). The second one covers the random losses $\left(Y_{1}, Y_{2}\right)$, also exposed to dependent individual risks denoted by $T_{1}$ and $T_{2}$. Moreover, there exists a common risk, with random loss $S$. If the policies cover only the largest loss, we have

$$
\left(X_{1}, X_{2}\right)=\left[\max \left(S_{1}, S\right), \max \left(S_{2}, S\right)\right] \quad \text { and } \quad\left(Y_{1}, Y_{2}\right)=\left[\max \left(T_{1}, S\right), \max \left(T_{2}, S\right)\right] .
$$

Suppose that for each of these two policies, the retained loss of the insurance company is some nondecreasing function $h_{i}($.$) of the random losses, i=1,2$, For the first policy its expected retained loss is given by $\mathrm{E}\left[h_{1}\left(X_{1}\right)+h_{2}\left(X_{2}\right)\right]$. Analogously, $\mathrm{E}\left[h_{1}\left(Y_{1}\right)+h_{2}\left(Y_{2}\right)\right]$ is the expected retained loss for the second policy. Under specifications in Example 4.3.8, the conditions of Lemma 4.3.6 hold true. Applying Corollary 4.3.7 we conclude that $\left(X_{1}, X_{2}\right) \leq_{s t}\left(Y_{1}, Y_{2}\right)$. Thus, we have

$$
\mathrm{E}\left[h_{1}\left(X_{1}\right)+h_{2}\left(X_{2}\right)\right] \leq \mathrm{E}\left[h_{1}\left(Y_{1}\right)+h_{2}\left(Y_{2}\right)\right]
$$

provided the expectations exist. Therefore, the expected retained loss is smaller in the first insurance policy than in the second one.

With additional assumptions in Lemma 4.3.6, we can obtain another related concordance order result for copulas of d-EMO distributions. We will consider the most general case, when two dEMO distributions are obtained from different pairs of random variables $\left(S_{1}, S_{2}\right)$ and $\left(T_{1}, T_{2}\right)$, and different common shocks $S_{3}$ and $T_{3}$. The result is presented in the next Lemma.

Lemma 4.3.10. Suppose $S_{i}$ and $T_{i}, i=1,2,3$, are continuous random variables and let $A_{S_{1}, S_{2}}\left(x_{1}, x_{2}\right)=\ln \frac{F_{S_{1}, S_{2}}\left(x_{1}, x_{2}\right)}{F_{S_{1}}\left(x_{1}\right) F_{S_{2}}\left(x_{2}\right)} \geq A_{T_{1}, T_{2}}\left(y_{1}, y_{2}\right)=\ln \frac{F_{T_{1}, T_{2}}\left(y_{1}, y_{2}\right)}{F_{T_{1}}\left(y_{1}\right) F_{T_{2}}\left(y_{2}\right)}$ for $x_{i} \leq y_{i}, i=1,2$. Define the d-EMO distributions

$$
\left(X_{1}, X_{2}\right)=\left[\max \left(S_{1}, S_{3}\right), \max \left(S_{2}, S_{3}\right)\right] \text { and }\left(Y_{1}, Y_{2}\right)=\left[\max \left(T_{1}, T_{3}\right), \max \left(T_{2}, T_{3}\right)\right] .
$$

If $S_{1} \leq_{s t} T_{1}, S_{2} \leq_{s t} T_{2}, S_{3} \geq_{s t} T_{3}$ and is verified that $X_{1} \leq_{s t} Y_{1}$ and $X_{2} \leq_{s t} Y_{2}$, then $C_{X_{1}, X_{2}}(u, v) \geq C_{Y_{1}, Y_{2}}(u, v)$ for all $u, v \in[0,1]$.

Proof. Since $F_{X_{i}}(x) \geq F_{Y_{i}}(x)$, then $F_{X_{i}}^{-1}(u) \leq F_{Y_{i}}^{-1}(u), i=1,2$. The result follows from the expression for the copula of d-EMO distributions (4.10) and Lemma 4.3.3.

Corollary 4.3.11. Under the assumptions of Lemma 4.3.10, $\left(X_{1}, X_{2}\right) \leq{ }_{s t}\left(Y_{1}, Y_{2}\right)$.

Proof. Since $X_{i} \leq_{s t} Y_{i}, i=1,2$, we have $F_{X_{i}}(x) \geq F_{Y_{i}}(x)$. Therefore

$$
\begin{aligned}
F_{X_{1}, X_{2}}\left(x_{1}, x_{2}\right) & =C_{X_{1}, X_{2}}\left(F_{X_{1}}\left(x_{1}\right), F_{X_{2}}\left(x_{2}\right)\right) \geq C_{X_{1}, X_{2}}\left(F_{Y_{1}}\left(x_{1}\right), F_{Y_{2}}\left(x_{2}\right)\right) \\
& \geq C_{Y_{1}, Y_{2}}\left(F_{Y_{1}}\left(x_{1}\right), F_{Y_{2}}\left(x_{2}\right)\right)=F_{Y_{1}, Y_{2}}\left(x_{1}, x_{2}\right) .
\end{aligned}
$$




\subsection{Concluding remarks}

A dual version of the generalized Marshall-Olkin of Li and Pellerey (2011) was introduced by Fang and Li (2013) motivated by its potential applications in Risk Analysis, Financial Engineering and Economics. In this chapter we extended the model of Fang and Li (2013) relaxing their assumption of independence between the subjacent individual shocks. In the proposed dual extended Marshall-Olkin distribution (4.7), the dependence structure between its two components is explained not only by the common shock, but also by the joint distribution of the individual shocks. We do believe that d-EMO model will be both of further theoretical and practical interest.

With the additional source of dependence, represented by the function $A_{T_{1}, T_{2}}\left(x_{1}, x_{2}\right)$ given by (4.6), the d-EMO distributions allow modeling both positive and negative quadrant dependence between its components. Moreover, the joint distribution may be non-exchangeable even if the marginals have the same distribution. These properties enrich and complement possibilities of the generalized version introduced by Fang and Li (2013) and overcomes its restrictive assumption of independence between the subjacent individual shocks.

A distributional property of inactivity times, similar to the one related with residual lifetimes in extended MO model and presented in Chapter 3, was demonstrated. Bounds for the joint distribution were obtained and several stochastic and concordance order results were reported with supporting examples.

Absolutely continuous version for d-EMO, bivariate reversed lack-of-memory (see Remark 2.4.2) and reliability properties as well as extreme value results can be obtained, following similar approach as in Chapter 3. We did not consider inference procedures related to the d-EMO model (4.7), nor application for real data set. These important issues, as well as multivariate extensions, are part of our future lines of research. 


\section{Chapter 5}

\section{Bivariate Distributions with Linear Sum of Hazard Gradient Components}

\subsection{Introduction}

In Chapter 1 we introduced the functional equation (1.2) as the defining relation for the univariate lack-of-memory property (LMP) of the exponential distribution. That equation admits the equivalent representation

$$
\mathbb{P}(X>x+t \mid X>t)=\mathbb{P}(X>x) \text { for all } x \geq 0, t \geq 0
$$

and plays a central role in survival analysis, reliability, insurance, finance and many other fields. Equation (5.1) tells us that the conditional distribution of $X-t$ given $\{X>t\}$ is independent of "age" $t$.

The same property is extended in a multivariate framework in Marshall and Olkin (1967). As shown in Chapter 2, in the bivariate case they look for continuous distributions such that relation (2.7) holds true, i.e.,

$$
\mathbb{P}\left(X_{1}>x_{1}+t, X_{2}>x_{2}+t \mid X_{1}>t, X_{2}>t\right)=\mathbb{P}\left(X_{1}>x_{1}, X_{2}>x_{2}\right)
$$

for all $x_{1}, x_{2} \geq 0$ and $t \geq 0$. They characterize the Marshall-Olkin bivariate exponential distribution with exponential marginals.

Remark 5.1.1. Marshall and Olkin (1967) demonstrate that, in relation (2.7), bivariate LMP, absolute continuity and exponential marginals cannot occur simultaneously except for bivariate distributions with independent exponential marginals.

The distribution of $X_{i}-t$ given $\left\{X_{i}>t\right\}, i=1,2$, generally depends on the "age" $t$ (since it is not necessarily exponential), whereas $X_{i}-t$ is independent on the same "age", when the conditioning set is $\left\{X_{1}>t, X_{2}>t\right\}$ as in relation (2.7). Therefore, do exist distributions satisfying (2.7) with non-exponential marginals. Apart from Marshall and Olkin's bivariate exponential distribution, other known solutions of (2.7) are the bivariate distributions obtained by Freund (1961), Block and Basu (1974), Proschan and Sullo (1976), Friday and Patil (1977) and all distributions shown in Kulkarni (2006), see Chapter 10 in Balakrishnan and Lai (2009) for related discussion as well. 
We view $\left(x_{1}, x_{2}\right)$ as the realization of a continuous random vector $\left(X_{1}, X_{2}\right)$ taking values on the first quadrant $\mathbf{R}_{+}^{2}=\left\{\left(x_{1}, x_{2}\right) \mid x_{1} \geq 0, x_{2} \geq 0\right\}$. Relation (2.7) can be equivalently rewritten as the functional equation (2.6), i.e.,

$$
S_{X_{1}, X_{2}}\left(x_{1}+t, x_{2}+t\right)=S_{X_{1}, X_{2}}\left(x_{1}, x_{2}\right) S_{X_{1}, X_{2}}(t, t)
$$

for all $x_{1}, x_{2} \geq 0$ and $t>0$ which is named bivariate lack-of-memory property, to be abbreviated $B L M P_{1}$.

Following Johnson and Kotz (1975) let us define the two components of the hazard gradient vector $\mathbf{R}\left(x_{1}, x_{2}\right)=\left(r_{1}\left(x_{1}, x_{2}\right), r_{2}\left(x_{1}, x_{2}\right)\right)$ as

$$
r_{i}\left(x_{1}, x_{2}\right)=-\frac{\partial}{\partial x_{i}} \ln S_{X_{1}, X_{2}}\left(x_{1}, x_{2}\right), i=1,2,
$$

which uniquely determine the joint distribution, see Marshall (1975). All distributions possessing $B L M P_{1}$ satisfy the equation $r_{1}\left(x_{1}, x_{2}\right)+r_{2}\left(x_{1}, x_{2}\right)=a_{0}$ where $a_{0}$ is a nonnegative constant, see Theorem 2 in Kulkarni (2006) for details.

In fact, Johnson and Kotz (1975) introduce another version of the bivariate LMP under the name local lack-of-memory property. They enforce conditional distributions $\left\{X_{1} \mid X_{2}>x_{2}\right\}$ and $\left\{X_{2} \mid X_{1}>x_{1}\right\}$ to possess (preserve) the univariate LMP defined by (5.1), see their sections $3(\mathrm{iv})$ and 5.4. The local LMP was rediscovered by Roy (2002a) and named $B L M P_{2}$. This property was identified by a local "constancy" of the conditional failure rates $r_{i}\left(x_{1}, x_{2}\right), i=1,2$, in Johnson and Kotz (1975), in the sense that $r_{i}\left(x_{1}, x_{2}\right)$ does not depend on $x_{i}, i=1,2$.

The only absolutely continuous distribution with such a property is the Gumbel's type I bivariate exponential distribution, given by (5.3). For Gumbel's bivariate exponential one gets $r_{i}\left(x_{1}, x_{2}\right)=$ $a_{0 i}+a_{1} x_{3-i}, i=1,2$, for some non-negative constants $a_{01}, a_{02}$ and $a_{1}$. Thus, substituting $a_{0}=$ $a_{01}+a_{02}$, the sum of the components of the hazard gradient vector is $r_{1}\left(x_{1}, x_{2}\right)+r_{2}\left(x_{1}, x_{2}\right)=$ $a_{0}+a_{1} x_{1}+a_{1} x_{2}$.

In this chapter we link $B L M P_{1}$ and $B L M P_{2}$ in a new class of bivariate continuous distributions (to be denoted by $\mathcal{L}(\mathbf{x} ; \mathbf{a})$ with $\mathbf{x}=\left(x_{1}, x_{2}\right)$ and $\left.\mathbf{a}=\left(a_{0}, a_{1}, a_{2}\right)\right)$, such that the sum $r_{1}\left(x_{1}, x_{2}\right)+$ $r_{2}\left(x_{1}, x_{2}\right)$ is a linear function of both arguments $x_{1}$ and $x_{2}$.

In Section 5.2 we formalize and characterize the class $\mathcal{L}(\mathbf{x} ; \mathbf{a})$. It will be also recognized that only certain marginal distributions are allowed. The corresponding restrictions for the marginal densities as well as the geometric interpretation of the class $\mathcal{L}(\mathbf{x} ; \mathbf{a})$ are presented. In Section 5.3 we suggest a method for constructing related bivariate distributions starting from marginal failure rates or from a member of the class $\mathcal{L}(\mathbf{x} ; \mathbf{a})$. We illustrate the methodology developed by typical examples. A detailed discussion, possible alternative approaches, multivariate extensions and conclusions finalize this chapter.

\subsection{Model formalization and characterization}

We will give first few necessary notations and facts. In what follows, the class of continuous nonnegative bivariate distributions with linear sum of components of the hazard gradient vector will be defined and characterized.

Let $X_{1}$ and $X_{2}$ be two nonnegative continuous random variables defined by their survival func- 
tions $S_{X_{i}}\left(x_{i}\right)=\mathbb{P}\left(X_{i}>x_{i}\right), x_{i} \geq 0$, densities $f_{X_{i}}\left(x_{i}\right)$ or failure rates $r_{X_{i}}\left(x_{i}\right)=\frac{f_{X_{i}}\left(x_{i}\right)}{S_{X_{i}}\left(x_{i}\right)}, i=1,2$. It is well known that the univariate failure rate uniquely determines the distribution of a random variable due to the fundamental relation

$$
S_{X_{i}}(x)=\exp \left\{-\int_{0}^{x} r_{X_{i}}(u) \mathrm{d} u\right\}=\exp \left\{-H_{X_{i}}(x)\right\}
$$

where $H_{X_{i}}(x)$ is the cumulative failure rate function, see Barlow and Proschan (1981).

Denote by $f_{X_{1}, X_{2}}\left(x_{1}, x_{2}\right)$ the bivariate density of $X_{1}$ and $X_{2}$. As a natural extension of failure rate in the bivariate case Basu (1971) suggested the relation $r_{X_{1}, X_{2}}\left(x_{1}, x_{2}\right)=\frac{f_{X_{1}, X_{2}}\left(x_{1}, x_{2}\right)}{S_{X_{1}, X_{2}}\left(x_{1}, x_{2}\right)}$. In general, the bivariate failure rate $r_{X_{1}, X_{2}}\left(x_{1}, x_{2}\right)$ does not uniquely determine the joint distribution of $X_{1}$ and $X_{2}$, consult Navarro (2008) for corresponding necessary conditions. So, complementary (conditional) characteristics should be taken into consideration. We will explore the conditional failure rates $r_{i}\left(x_{1}, x_{2}\right), i=1,2$, being the two components of the hazard gradient vector $\mathbf{R}\left(x_{1}, x_{2}\right)=$ $\left(r_{1}\left(x_{1}, x_{2}\right), r_{2}\left(x_{1}, x_{2}\right)\right)$.

\subsubsection{Model specification}

We begin with the definition of the class $\mathcal{L}(\mathbf{x} ; \mathbf{a})$.

Definition 5.2.1. The class $\mathcal{L}(\mathbf{x} ; \mathbf{a})$ is composed by nonnegative bivariate continuous distributions that satisfy the relation

$$
r\left(x_{1}, x_{2}\right)=r_{1}\left(x_{1}, x_{2}\right)+r_{2}\left(x_{1}, x_{2}\right)=a_{0}+a_{1} x_{1}+a_{2} x_{2}
$$

for all $x_{1}, x_{2} \geq 0$, where $\mathbf{x}=\left(x_{1}, x_{2}\right)$ and $\mathbf{a}=\left(a_{0}, a_{1}, a_{2}\right)$ is the parameter vector with nonnegative elements.

In other words, the class $\mathcal{L}(\mathbf{x} ; \mathbf{a})$ is composed by nonnegative bivariate continuous distributions such that the sum of the components of hazard gradient vector is a linear function of both arguments $x_{1}$ and $x_{2}$. Detailed restrictions of constants $a_{0}, a_{1}$ and $a_{2}$ will be given in Theorem 5.2.14 and Proposition 5.2.17.

Remark 5.2.2. Note that the class $\mathcal{L}(\mathbf{x} ; \mathbf{a})$ may include distributions with a singularity along the line $x_{1}=x_{2}=x \geq 0$, where the conditional failure rates $r_{1}\left(x_{1}, x_{2}\right)$ and $r_{2}\left(x_{1}, x_{2}\right)$ will not be defined. In such cases one should consider the rule $r(x, x)=-\frac{d}{d x} \ln S_{X_{1}, X_{2}}(x, x)$, see related comments in Remarks 5.2.4 and 5.2.7.

To understand the physical meaning of the sum $r_{1}\left(x_{1}, x_{2}\right)+r_{2}\left(x_{1}, x_{2}\right)$ one needs knowledge about directional derivative for a function $s: \mathbf{R}^{m} \rightarrow \mathbf{R}^{n}$ when $m=2$ and $n=1$, consult page 42 in Munkres (1991), for example.

Definition 5.2.3 (Munkres (1991)). Given set $\mathbf{C} \subset \mathbf{R}^{2}=[-\infty, \infty]^{2}$, consider the function $s$ : $\mathbf{C} \rightarrow \mathbf{R}$ and suppose $\mathbf{C}$ contains a neighborhood of $\mathbf{x}=\left(x_{1}, x_{2}\right) \in \mathbf{C}$. Given the non-zero vector $\mathbf{u}=\left(u_{1}, u_{2}\right) \in R^{2}$ the directional derivative of $s(.,$.$) at \mathbf{x}$ with respect to the vector $\mathbf{u}$ is given by

$$
s^{\prime}(\mathbf{x} ; \mathbf{u})=\lim _{z \rightarrow 0} \frac{s\left(\left(x_{1}, x_{2}\right)+z\left(u_{1}, u_{2}\right)\right)-s\left(\left(x_{1}, x_{2}\right)\right)}{z},
$$

provided the limit exists. 
Remark 5.2.4 (Directional derivative). Directional derivatives at a point $\mathbf{x}$ with respect to a vector $\mathbf{u}$ can be easily computed when the function $s(.,$.$) is differentiable at \left(x_{1}, x_{2}\right)$. In such cases we have

$$
s^{\prime}(\mathbf{x} ; \mathbf{u})=\frac{\partial}{\partial x_{1}} s\left(x_{1}, x_{2}\right) u_{1}+\frac{\partial}{\partial x_{2}} s\left(x_{1}, x_{2}\right) u_{2},
$$

see Theorem 5.1 at page 44 in Munkres (1991). We are particularly interested when $\mathbf{u}=(1,1)$, i.e. we get

$$
s^{\prime}\left(\left(x_{1}, x_{2}\right) ;(1,1)\right)=\frac{\partial}{\partial x_{1}} s\left(x_{1}, x_{2}\right)+\frac{\partial}{\partial x_{2}} s\left(x_{1}, x_{2}\right) .
$$

Thus, the directional derivative of $s(.,$.$) at \left(x_{1}, x_{2}\right)$ with respect to the vector $(1,1)$ is given by the sum of the corresponding partial derivatives.

Substituting $-\ln \left[S_{X_{1}, X_{2}}\left(x_{1}, x_{2}\right)\right]=s\left(x_{1}, x_{2}\right)$ in the right hand side of last equation we obtain the sum of elements of the hazard gradient vector $\mathbf{R}\left(x_{1}, x_{2}\right)$. The interpretation is that wherever $S_{X_{1}, X_{2}}\left(x_{1}, x_{2}\right)$ is differentiable, the sum $r_{1}\left(x_{1}, x_{2}\right)+r_{2}\left(x_{1}, x_{2}\right)$ establishes the performance of the joint survival function $S_{X_{1}, X_{2}}\left(x_{1}, x_{2}\right)$ along the lines parallel to $\left\{x_{1}=x_{2}=x \geq 0\right\}$, i.e. having $45^{0}$ inclination.

The next theorem, numbered Theorem 6.2 at page 50 in Munkres (1991), will be necessary in characterization Theorem 5.2.11.

Theorem 5.2.5. [Munkres (1991)] Let $\mathbf{A}$ be an open set in $\mathbf{R}^{2}$. Suppose the partial derivatives of the function $s$ exist at each point $\mathbf{x}=\left(x_{1}, x_{2}\right) \in \mathbf{A}$ and are continuous. Then $s$ is differentiable at each point of $\mathbf{A}$.

Remark 5.2.6. A function satisfying the hypothesis of Theorem 5.2.5 is often said to be continuously differentiable, or of class $C^{1}$ on $\mathbf{A}$.

Remark 5.2.7. Combining Theorems 5.1 and 6.2 in Munkres (1991), whenever the partial derivatives of the function s exist and are continuous at each point $\left(x_{1}, x_{2}\right)$ of an open set $\mathbf{A}$ belonging to the domain of $s$, the directional derivative of $s(.,$.$) at \left(x_{1}, x_{2}\right) \in \mathbf{A}$ with respect to the vector $(1,1)$ is given by the sum of the partial derivatives.

Now, let us analyse the relation (5.2) from completely different point of view. Define the function

$$
\psi\left(x_{1}, x_{2}\right)=r\left(x_{1}, x_{2}\right)-a_{0} \quad \text { and set } \quad A_{1}\left(x_{1}\right)=\psi\left(x_{1}, 0\right), A_{2}\left(x_{2}\right)=\psi\left(0, x_{2}\right) .
$$

The linear functions $A_{i}\left(x_{i}\right)=a_{i} x_{i}$ satisfy $A_{i}\left(x_{i}+y_{i}\right)=A_{i}\left(x_{i}\right)+A_{i}\left(y_{i}\right)$ for all $x_{i}, y_{i} \geq 0, i=1,2$, i.e. $A_{i}(x)$ are additive functionals and only continuous solutions of Cauchy functional equation $f(x+y)=f(x)+f(y)$, see Theorem 1.1 in Sahoo and Kannappan (2011). In fact, we arrive to the following statement.

Lemma 5.2.8. The class $\mathcal{L}(\mathbf{x} ; \mathbf{a})$ of nonnegative bivariate continuous distributions specified by relation (5.2) can be equivalently defined by linear (additive) functionals

$$
A_{1}\left(x_{1}\right)=r\left(x_{1}, 0\right)-a_{0} \quad \text { and } \quad A_{2}\left(x_{2}\right)=r\left(0, x_{2}\right)-a_{0}
$$

being the only continuous solutions of the functional equation $f(x+y)=f(x)+f(y)$. 
Bivariate distributions following the property (5.2) do exist. From our discussion in Section 5.1 we already know that Marshall-Olkin's bivariate exponential, given by (2.4), and Gumbel's type I bivariate exponential distribution, given by (5.3), satisfy relation (5.2). Distributions with independent marginals will be discussed in Section 5.3.1. Few typical examples that will be explored in the exposition are considered in Remark 5.2.9.

Remark 5.2.9 (Few members of the class $\mathcal{L}(\mathbf{x} ; \mathbf{a})$ ). Here we will give five examples.

- From Section 2.3, one obvious extension of the univariate LMP to the bivariate case is given by relation (2.5), i.e.,

$$
S_{X_{1}, X_{2}}\left(x_{1}+y_{1}, x_{2}+y_{2}\right)=S_{X_{1}, X_{2}}\left(x_{1}, x_{2}\right) S_{X_{1}, X_{2}}\left(y_{1}, y_{2}\right)
$$

for all $x_{1}, x_{2}, y_{1}, y_{2} \geq 0$. The only solution of this functional equation is given by

$$
S_{X_{1}, X_{2}}\left(x_{1}, x_{2}\right)=\exp \left\{-b_{1} x_{1}-b_{2} x_{2}\right\}, \quad \text { for } \quad b_{1}, b_{2}>0 \text {, }
$$

i.e. $X_{1}$ and $X_{2}$ are independent and exponentially distributed with parameters $b_{1}$ and $b_{2}$. One can get this (absolutely continuous) case from (5.2) by setting $a_{0}=b_{1}+b_{2}$ and $a_{1}=a_{2}=0$;

- Let $a_{1}=a_{2}=0$ in (5.2). Hence, we obtain all bivariate distributions shown in Kulkarni (2006) having a constant sum $a_{0}$ of the hazard gradient components, i.e. $r_{1}\left(x_{1}, x_{2}\right)+r_{2}\left(x_{1}, x_{2}\right)=$ $a_{0}>0$. These distributions possess the BLMP $P_{1}$ given by the functional equation (2.6).

In fact, the marginal distributions treated by Kulkarni (2006) do not need to have a constant failure rate (i.e. to be exponential), but the corresponding bivariate distributions satisfy (2.6). As a particular case, when the marginal failure rates are constant, one gets the classical bivariate Marshall-Olkin (MO) exponential distribution given by (2.4), i.e.,

$$
S_{X_{1}, X_{2}}\left(x_{1}, x_{2}\right)=\exp \left\{-\lambda_{1} x_{1}-\lambda_{2} x_{2}-\lambda_{3} \max \left(x_{1}, x_{2}\right),\right\} \quad x_{1}, x_{2} \geq 0
$$

exhibiting singularity along the line $x_{1}=x_{2}=x \geq 0$, where $\lambda_{i}>0, i=1,2,3$.

It is well known that MO distributions are characterized by the stochastic representation (2.9), i.e.,

$$
\left(X_{1}, X_{2}\right)=\left[\min \left(T_{1}, T_{3}\right), \min \left(T_{2}, T_{3}\right)\right]
$$

(i.e. by the Marshall-Olkin "fatal" shock model), where $T_{i}$ are independent exponentially distributed random variables with parameters $\lambda_{i}>0, i=1,2,3$, see Section 2.3;

- The absolutely continuous Gumbel's type I bivariate exponential distribution

$$
S_{X_{1}, X_{2}}\left(x_{1}, x_{2}\right)=\exp \left\{-\lambda_{1} x_{1}-\lambda_{2} x_{2}-\theta \lambda_{1} \lambda_{2} x_{1} x_{2}\right\}, \quad x_{1}, x_{2} \geq 0
$$

is a result of substituting $a_{0}=\lambda_{1}+\lambda_{2}$ and $a_{1}=a_{2}=\theta \lambda_{1} \lambda_{2}$ in (5.2) where $\lambda_{i}>0, i=1,2$ and $\theta \in[0,1]$. This distribution does not have a singular part, of course. It is negative quadrant dependent since $S_{X_{1}, X_{2}}\left(x_{1}, x_{2}\right)<S_{X_{1}}\left(x_{1}\right) S_{X_{2}}\left(x_{2}\right)$ for all $x_{1}, x_{2} \geq 0$.

As we noted in Introduction, the Gumbel's distribution represented by (5.3) is characterized by $B L M P_{2}$, which requires that conditional distributions of $\left\{X_{1} \mid X_{2}>x_{2}\right\}$ and $\left\{X_{2} \mid X_{1}>x_{1}\right\}$ 
should preserve the univariate lack-of-memory property, i.e.,

$$
\mathbb{P}\left(X_{i}>x_{i}+y_{i} \mid X_{j}>x_{j}\right)=\mathbb{P}\left(X_{i}>x_{i} \mid X_{j}>x_{j}\right) \mathbb{P}\left(X_{i}>y_{i} \mid X_{j}>x_{j}\right)
$$

for $i=1,2$ and $j=3-i$. These conditions are equivalent to impose that a bivariate distribution possesses the $B L M P_{2}$ if and only if both relations

$$
S_{X_{1}, X_{2}}\left(x_{1}+y_{1}, x_{2}\right) S_{X_{1}, X_{2}}\left(0, x_{2}\right)=S_{X_{1}, X_{2}}\left(x_{1}, x_{2}\right) S_{X_{1}, X_{2}}\left(y_{1}, x_{2}\right)
$$

and

$$
S_{X_{1}, X_{2}}\left(x_{1}, x_{2}+y_{2}\right) S_{X_{1}, X_{2}}\left(x_{1}, 0\right)=S_{X_{1}, X_{2}}\left(x_{1}, x_{2}\right) S_{X_{1}, X_{2}}\left(x_{1}, y_{2}\right)
$$

are satisfied for all choices of non-negative $x_{1}, x_{2}, y_{1}$ and $y_{2}$;

- The bivariate Schur-constant law $S_{X_{1}, X_{2}}\left(x_{1}, x_{2}\right)=\exp \left\{-x_{1}-x_{2}\right\}$ can be obtained letting $a_{0}=2$ and $a_{1}=a_{2}=0$ in (5.2). Barlow and Mendel (1992) have characterized it in terms of bivariate no-aging property (used in Bayesian reliability analysis) by relation

$$
\mathbb{P}\left(X_{1}>x_{1}+t \mid X_{1}>x_{1}, X_{2}>x_{2}\right)=\mathbb{P}\left(X_{2}>x_{2}+t \mid X_{1}>x_{1}, X_{2}>x_{2}\right),
$$

see Nair and Sankaran (2013) as well. In fact, the last equation is a particular case of (5.4), i.e. valid for exchangeable distributions (which should have the same marginals) possessing the local lack-of-memory property;

- If we set $a_{0}=\lambda_{3}>0, a_{1}=2 \lambda_{1}>0$ and $a_{2}=2 \lambda_{2}>0$ in (5.2) we will obtain the joint survival function

$$
S_{X_{1}, X_{2}}\left(x_{1}, x_{2}\right)=\exp \left\{-\lambda_{1} x_{1}^{2}-\lambda_{2} x_{2}^{2}-\lambda_{3} \max \left(x_{1}, x_{2}\right)\right\}, \quad x_{1}, x_{2} \geq 0,
$$

which has a singular component along the line $x_{1}=x_{2}=x \geq 0$ and satisfies the stochastic representation (2.9). The distribution given by (5.5) is a member of the generalized MarshallOlkin (GMO) distributions introduced by Li and Pellerey (2011). The random variables $T_{1}, T_{2}$ and $T_{3}$ in (2.9) are assumed to be independent in the class of GMO distributions, relaxing $M O$ assumption of exponential marginals, see Section 3.1. It is direct to check that $S_{X_{1}, X_{2}}\left(x_{1}, x_{2}\right)>$ $S_{X_{1}}\left(x_{1}\right) S_{X_{2}}\left(x_{2}\right)$ for all $x_{1}, x_{2} \geq 0$, i.e the bivariate distribution in (5.5) is positive quadrant dependent.

Therefore, one can find many examples of bivariate distributions possessing the $B L M P_{1}$ and $B L M P_{2}$ represented by (2.6) and (5.4), respectively, as well as those exhibiting positive or negative quadrant dependence that belong to the class $\mathcal{L}(\mathbf{x} ; \mathbf{a})$, satisfy $(5.2)$, or the equivalent condition in Lemma 5.2.8 related to Cauchy functional equation. 


\subsubsection{A characterization}

The hazard gradient vector $\mathbf{R}\left(x_{1}, x_{2}\right)=\left(r_{1}\left(x_{1}, x_{2}\right), r_{2}\left(x_{1}, x_{2}\right)\right)$ uniquely determines the bivariate distribution by means of the line integral

$$
S_{X_{1}, X_{2}}\left(x_{1}, x_{2}\right)=\exp \left\{-\int_{\mathcal{C}} \mathbf{R}(\mathbf{z}) \cdot \mathrm{d} \mathbf{z}\right\}
$$

where $\mathcal{C}$ is a sufficiently smooth continuous path beginning at $(0,0)$ and finishing at $\left(x_{1}, x_{2}\right)$.

Remark 5.2.10. In particular, when $S_{X_{1}, X_{2}}\left(x_{1}, x_{2}\right)$ is continuous, the hazard gradient vector is useful even when it does not exist everywhere on the support of $S_{X_{1}, X_{2}}\left(x_{1}, x_{2}\right)$, i.e., on the set $\left\{\left(x_{1}, x_{2}\right) \in \mathbf{R}_{+}^{2} \mid S_{X_{1}, X_{2}}\left(x_{1}, x_{2}\right)>0\right\}$, where $\mathbf{R}_{+}^{2}$ is the first quadrant. In fact, the above expression holds true provided along the path of integration $\mathcal{C}$ we have $\int_{\mathcal{C}} \mathbf{R}(\mathbf{z}) \cdot \mathrm{d} \mathbf{z}$ absolutely continuous and $\mathbf{R}\left(x_{1}, x_{2}\right)$ exists almost everywhere, see Marshall (1975). We make use of this property and consider integration paths $\mathcal{C}$ that do not intercept the set $\left\{x_{1}=x_{2}=x \geq 0\right\}$, where it may happen the joint survival function possess a singular component.

The next characterization theorem holds for bivariate distributions belonging to the class $\mathcal{L}(\mathbf{x} ; \mathbf{a})$, whose survival functions are continuous and possess continuous first partial derivatives (and hence hazard gradient vector $\left.\mathbf{R}\left(x_{1}, x_{2}\right)\right)$. A possible anomaly (singularity) that may happen when $x_{1}=$ $x_{2}=x \geq 0$ is discussed in Remark 5.2.12.

Theorem 5.2.11. If $S_{X_{1}, X_{2}}\left(x_{1}, x_{2}\right)$ is continuous with continuous first partial derivatives, relation (5.2) is fulfilled if and only if the corresponding joint survival function can be represented by

$$
S_{X_{1}, X_{2}}\left(x_{1}, x_{2}\right)= \begin{cases}S_{X_{1}}\left(x_{1}-x_{2}\right) \exp \left\{-a_{0} x_{2}-a_{1} x_{1} x_{2}-\frac{a_{2}-a_{1}}{2} x_{2}^{2}\right\}, & \text { if } x_{1} \geq x_{2} \geq 0 \\ S_{X_{2}}\left(x_{2}-x_{1}\right) \exp \left\{-a_{0} x_{1}-a_{2} x_{1} x_{2}-\frac{a_{1}-a_{2}}{2} x_{1}^{2}\right\}, & \text { if } x_{2} \geq x_{1} \geq 0\end{cases}
$$

Proof. From Remarks 5.2.7 and 5.2.10, the sum of the components of hazard gradient equals the directional derivative of $-\ln S_{X_{1}, X_{2}}\left(x_{1}, x_{2}\right)$ along the direction of the vector $(1,1)$ as well as the hazard gradient vector can be used to recover the corresponding survival function.

Assume (5.2) is satisfied. Consider a sufficiently smooth continuous path (curve) $\mathcal{C}$ beginning at point $(0,0)$ and finishing at point $\left(x_{1}, x_{2}\right)$ in $\mathbf{R}_{+}^{2}$. Suppose that along this path $S_{X_{1}, X_{2}}\left(x_{1}, x_{2}\right)$ is absolutely continuous and hazard components $r_{i}\left(x_{1}, x_{2}\right), i=1,2$ do exist almost everywhere in the set $\mathcal{C}$.

Let us parameterize the curve $\mathcal{C}$ by smooth functions $y_{i}(t)$ for $t \in[a, b]$, such that $y_{i}(a)=0$ and $y_{i}(b)=x_{i}, i=1,2$. Now, using the path independence property of line integrals, define the function $R_{X_{1}, X_{2}}\left(x_{1}, x_{2}\right)$ as

$$
R_{X_{1}, X_{2}}\left(x_{1}, x_{2}\right)=\int_{\mathcal{C}} \mathbf{R}(\mathbf{z}) \cdot \mathrm{d} \mathbf{z}=\int_{a}^{b} r_{1}\left(y_{1}(t), y_{2}(t)\right) y_{1}^{\prime}(t) \mathrm{d} t+\int_{a}^{b} r_{2}\left(y_{1}(t), y_{2}(t)\right) y_{2}^{\prime}(t) \mathrm{d} t .
$$

Following Marshall (1975), we have

$$
S_{X_{1}, X_{2}}\left(x_{1}, x_{2}\right)=\exp \left\{-R_{X_{1}, X_{2}}\left(x_{1}, x_{2}\right)\right\}
$$

Initially let $x_{1} \geq x_{2} \geq 0$ and choose a particular path $\mathcal{C}$ from $(0,0)$ to $\left(x_{1}, x_{2}\right)$ as the union of two line segments: the first linking $(0,0)$ with $\left(x_{1}-x_{2}, 0\right)$ and the second joining $\left(x_{1}-x_{2}, 0\right)$ with 
$\left(x_{1}, x_{2}\right)$. Denote these line segments by $\mathcal{C}_{1}$ and $\mathcal{C}_{2}$, respectively, see Figure 5.1 . Notice that curves $\mathcal{C}_{1}$ and $\mathcal{C}_{2}$, can be parameterized as follows

$$
\mathcal{C}_{1}=\left\{\left(y_{1}(t), y_{2}(t)\right) \in \mathbf{R}_{+}^{2} \text { such that }\left(y_{1}(t), y_{2}(t)\right)=(t, 0), \text { with } t \in\left[0, x_{1}-x_{2}\right]\right\}
$$

and

$$
\mathcal{C}_{2}=\left\{\left(y_{1}(t), y_{2}(t)\right) \in \mathbf{R}_{+}^{2} \text { such that }\left(y_{1}(t), y_{2}(t)\right)=\left(x_{1}-x_{2}+t, t\right) \text {, with } t \in\left[0, x_{2}\right]\right\} .
$$

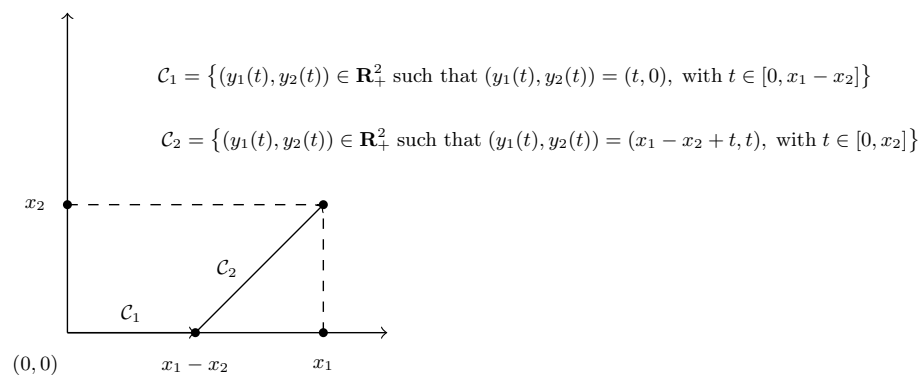

Figure 5.1: Paths of integration $\mathcal{C}_{1}$ and $\mathcal{C}_{2}$ when $x_{1} \geq x_{2} \geq 0$.

Along the path $\mathcal{C}_{1}$ we have $S_{X_{1}, X_{2}}\left(x_{1}, x_{2}\right)=S_{X_{1}, X_{2}}\left(x_{1}, 0\right)=S_{X_{1}}\left(x_{1}\right)$ and therefore $r_{1}\left(x_{1}, 0\right)=$ $r_{X_{1}}\left(x_{1}\right)$ together with $y_{2}^{\prime}(t)=0$. Applying (5.7) along the path $\mathcal{C}_{1}$ with $y_{1}^{\prime}(t)=1$ gives

$$
R_{X_{1}, X_{2}}\left(x_{1}-x_{2}, 0\right)=\int_{0}^{x_{1}-x_{2}} r_{X_{1}}(t) \mathrm{d} t=H_{X_{1}}\left(x_{1}-x_{2}\right) .
$$

Notice that along the line segment $\mathcal{C}_{2}$ we have $y_{2}(t)=t$ and $y_{1}(t)=x_{1}-x_{2}+t$, i.e. $y_{1}^{\prime}(t)=$ $y_{2}^{\prime}(t)=1$. The use of (5.7) and relation (5.2) implies

$$
\begin{aligned}
R_{X_{1}, X_{2}}\left(x_{1}, x_{2}\right)-R_{X_{1}, X_{2}}\left(x_{1}-x_{2}, 0\right) & =\int_{0}^{x_{2}}\left[r_{1}\left(x_{1}-x_{2}+t, t\right)+r_{2}\left(x_{1}-x_{2}+t, t\right)\right] \mathrm{d} t \\
& =\int_{0}^{x_{2}}\left[a_{0}+a_{1}\left(x_{1}-x_{2}+t\right)+a_{2} t\right] \mathrm{d} t .
\end{aligned}
$$

Taking into account the representation (5.8) we obtain

$$
\begin{aligned}
S_{X_{1}, X_{2}}\left(x_{1}, x_{2}\right) & =\exp \left\{-H_{X_{1}}\left(x_{1}-x_{2}\right)-\int_{0}^{x_{2}}\left[a_{0}+a_{1}\left(x_{1}-x_{2}+t\right)+a_{2} t\right] \mathrm{d} t\right\} \\
& =S_{X_{1}}\left(x_{1}-x_{2}\right) \exp \left\{-\int_{0}^{x_{2}}\left[a_{0}+a_{1}\left(x_{1}-x_{2}+t\right)+a_{2} t\right] \mathrm{d} t\right\} .
\end{aligned}
$$

Finally, solving the integral in the foregoing equation we get

$$
S_{X_{1}, X_{2}}\left(x_{1}, x_{2}\right)=S_{X_{1}}\left(x_{1}-x_{2}\right) \exp \left\{-a_{0} x_{2}-a_{1} x_{1} x_{2}-\frac{a_{2}-a_{1}}{2} x_{2}^{2}\right\} .
$$

By analogy, for $x_{2} \geq x_{1} \geq 0$ we obtain

$$
S_{X_{1}, X_{2}}\left(x_{1}, x_{2}\right)=S_{X_{2}}\left(x_{2}-x_{1}\right) \exp \left\{-a_{0} x_{1}-a_{2} x_{1} x_{2}-\frac{a_{1}-a_{2}}{2} x_{1}^{2}\right\} .
$$

and necessary part of the statement is proved. 
Now suppose that the joint survival $S_{X_{1}, X_{2}}\left(x_{1}, x_{2}\right)$ function is given by (5.6). Direct calculus show that hazard components $r_{i}\left(x_{1}, x_{2}\right)=-\frac{\partial}{\partial x_{i}} \ln S_{X_{1}, X_{2}}\left(x_{1}, x_{2}\right), i=1,2$ should satisfy the following relations

$$
r_{1}\left(x_{1}, x_{2}\right)= \begin{cases}r_{X_{1}}\left(x_{1}-x_{2}\right)+a_{1} x_{2}, & \text { if } x_{1} \geq x_{2} \geq 0 \\ a_{0}+\left(a_{1}-a_{2}\right) x_{1}+a_{2} x_{2}-r_{X_{2}}\left(x_{2}-x_{1}\right), & \text { if } 0 \leq x_{1} \leq x_{2}\end{cases}
$$

and

$$
r_{2}\left(x_{1}, x_{2}\right)= \begin{cases}a_{0}+a_{1} x_{1}+\left(a_{2}-a_{1}\right) x_{2}-r_{X_{1}}\left(x_{1}-x_{2}\right), & \text { if } x_{1} \geq x_{2} \geq 0, \\ r_{X_{2}}\left(x_{2}-x_{1}\right)+a_{2} x_{1}, & \text { if } 0 \leq x_{1} \leq x_{2} .\end{cases}
$$

This means that $r_{1}\left(x_{1}, x_{2}\right)+r_{2}\left(x_{1}, x_{2}\right)=a_{0}+a_{1} x_{1}+a_{2} x_{2}$ for all $x_{1}, x_{2} \geq 0$, i.e. condition (5.2) is fulfilled. This completes the proof.

Remark 5.2.12 (Hazard vector elements in singularity case). The bivariate survival functions considered in Theorem 5.2.11 are not necessarily absolutely continuous (and therefore not differentiable almost everywhere). We do not exclude the possibility of existence of a singular component along the line $x_{1}=x_{2}=x \geq 0$ in our model, i.e. it may happen that $\mathbb{P}\left(X_{1}=X_{2}\right)>0$. Therefore, in the presence of such a singular component, the function $S_{X_{1}, X_{2}}\left(x_{1}, x_{2}\right)$ is not differentiable on the set $\Omega=\left\{\left(x_{1}, x_{2}\right) \in \mathbf{R}_{+}^{2} \mid x_{1}=x_{2}=x\right\}$. In this case the hazard gradient vector $\mathbf{R}\left(x_{1}, x_{2}\right)$ does not exist along the line $x_{1}=x_{2} x=\geq 0$. Hence, (5.9) and (5.10) have to be rewritten as follows

$$
r_{1}\left(x_{1}, x_{2}\right)= \begin{cases}r_{X_{1}}\left(x_{1}-x_{2}\right)+a_{1} x_{2}, & \text { if } x_{1}>x_{2} \geq 0, \\ \text { does not exist, } & \text { if } x_{1}=x_{2} \geq 0, \\ a_{0}+\left(a_{1}-a_{2}\right) x_{1}+a_{2} x_{2}-r_{X_{2}}\left(x_{2}-x_{1}\right), & \text { if } 0 \leq x_{1}<x_{2}\end{cases}
$$

and

$$
r_{2}\left(x_{1}, x_{2}\right)= \begin{cases}a_{0}+a_{1} x_{1}+\left(a_{2}-a_{1}\right) x_{2}-r_{X_{1}}\left(x_{1}-x_{2}\right), & \text { if } x_{1}>x_{2} \geq 0, \\ \text { does not exist, } & \text { if } x_{1}=x_{2} \geq 0, \\ r_{X_{2}}\left(x_{2}-x_{1}\right)+a_{2} x_{1}, & \text { if } 0 \leq x_{1}<x_{2} .\end{cases}
$$

Thus, taking into account Remark 5.2.2, one has to use a simple univariate derivative of $-\ln S_{X_{1}, X_{2}}(x, x)$ with respect to $x$ instead of the sum of partial derivatives.

We finish this subsection with the following

Remark 5.2.13 (Particular cases). Let us consider once more the following three models:

- Substituting $a_{1}=a_{2}=0$ in (5.2) and (5.6) (or/and in (5.9) and (5.10)), we obtain the characterization of all bivariate distributions specified in Theorem 2 and Corollary 1 in Kulkarni (2006);

- Let marginals be exponentially distributed, $\mathbb{P}\left(X_{1}=X_{2}\right)=0$ and $a_{1}=a_{2}$. It is direct to verify from (5.9) that $r_{1}\left(x_{1}, x_{2}\right)=r_{1}\left(0, x_{2}\right)$ being locally constant with respect to $x_{1}$. A similar treatment of (5.10) ensures local constancy of $r_{2}\left(x_{1}, x_{2}\right)$ with respect to $x_{2}$, i.e $r_{2}\left(x_{1}, x_{2}\right)=$ $r_{2}\left(x_{1}, 0\right)$. As we noted in Introduction, both relations characterize the BLMP $P_{2}$ and Gumbel's bivariate distribution (5.3) when $S_{X_{1}, X_{2}}\left(x_{1}, x_{2}\right)$ is absolutely continuous, see again sections $3(\mathrm{iv})$ and 5.4 in Johnson and Kotz (1975).; 
- Consider bivariate Schur-constant distribution in its general form

$$
S_{X_{1}, X_{2}}\left(x_{1}, x_{2}\right)=S\left(x_{1}+x_{2}\right), \text { where } S(x)=S_{X_{1}}(x)=S_{X_{2}}(x) .
$$

We obtain $r_{X_{1}}(x)=r_{X_{2}}(x)$ and $r_{i}\left(x_{1}, x_{2}\right)=r_{X_{i}}\left(x_{1}+x_{2}\right), i=1,2$. Because of the exchangeability of the model we get $a_{1}=a_{2}=a$ in (5.2) and the joint survival function can be written as

$$
S_{X_{1}, X_{2}}\left(x_{1}, x_{2}\right)=\exp \left\{-\frac{a_{0}}{2}\left(x_{1}+x_{2}\right)-\frac{a}{4}\left(x_{1}+x_{2}\right)^{2}\right\}
$$

for all $x_{1}, x_{2} \geq 0$.

\subsubsection{Restrictions on the marginal densities}

Theorem 5.2.11 characterizes bivariate distributions belonging to the class $\mathcal{L}(\mathbf{x} ; \mathbf{a})$, i.e. having joint survival function specified by relation (5.6), which imply some more restrictions on the margins of these distributions. In other words, $S_{X_{1}, X_{2}}\left(x_{1}, x_{2}\right)$ in (5.6) is a valid survival function only for certain marginal distributions of $X_{1}$ and $X_{2}$. The next result shows the corresponding constraints in terms of marginal densities for nonnegative $a_{1}$ and $a_{2}$ when $a_{1}+a_{2}>0$. The case $a_{1}=a_{2}=0$ is detailed studied by Kulkarni (2006).

Theorem 5.2.14. Let $X_{i}$ be a random variable with absolutely continuous density $f_{X_{i}}\left(x_{i}\right), i=1,2$. Then $S_{X_{1}, X_{2}}\left(x_{1}, x_{2}\right)$ in (5.6) is a proper bivariate survival function if and only if there exist nonnegative constants $a_{0}, a_{1}$ and $a_{2}$ with $a_{1}+a_{2}>0$, such that

$$
A\left(x_{i}, x_{j}\right)-a_{i} x_{j}+\frac{d}{d x_{i}} \log f_{X_{i}}\left(x_{i}-x_{j}\right)+a_{i}\left[x_{j} A\left(x_{i}, x_{j}\right)-1\right] \frac{S_{X_{i}}\left(x_{i}-x_{j}\right)}{f_{X_{i}}\left(x_{i}-x_{j}\right)} \geq 0
$$

where $A\left(x_{i}, x_{j}\right)=a_{0}+a_{i} x_{i}+\left(a_{j}-a_{i}\right) x_{j}$ for all $x_{i} \geq x_{j} \geq 0, i \neq j, i, j=1,2$. In addition

$$
\left[f_{X_{1}}(0)+f_{X_{2}}(0)-a_{0}\right] \sqrt{\frac{\pi}{2\left(a_{1}+a_{2}\right)}} \exp \left\{\frac{a_{0}^{2}}{2\left(a_{1}+a_{2}\right)}\right\}\left[1-\operatorname{Erf}\left(\frac{a_{0}}{\sqrt{2\left(a_{1}+a_{2}\right)}}\right)\right] \in[0,1] .
$$

Proof. Let $S_{X_{1}, X_{2}}\left(x_{1}, x_{2}\right)$ be given by (5.6). Then, the condition

$$
\frac{\partial^{2}}{\partial x_{1} \partial x_{2}} S_{X_{1}, X_{2}}\left(x_{1}, x_{2}\right) \geq 0
$$

after some algebra transforms into the inequality (5.11).

Since $S_{X_{1}, X_{2}}\left(x_{1}, x_{2}\right)$ may have a singular component along the line $x_{1}=x_{2}=x \geq 0$, then let $\alpha=\mathbb{P}\left(X_{1}=X_{2}\right) \in[0,1]$. The bivariate survival function $S_{X_{1}, X_{2}}\left(x_{1}, x_{2}\right)$ in (5.6) will be proper if and only if both the absolutely continuous part $S_{X_{1}, X_{2}}^{a c}\left(x_{1}, x_{2}\right)$ and the singular part $S_{X_{1}, X_{2}}^{s i}\left(x_{1}, x_{2}\right)$ are survival functions and from (2.11)

$$
S_{X_{1}, X_{2}}\left(x_{1}, x_{2}\right)=(1-\alpha) S_{X_{1}, X_{2}}^{a c}\left(x_{1}, x_{2}\right)+\alpha S_{X_{1}, X_{2}}^{s i}\left(\max \left\{x_{1}, x_{2}\right\}\right)
$$

for $\alpha \in[0,1]$. An equivalent expression in terms of joint densities is given by

$$
f_{X_{1}, X_{2}}\left(x_{1}, x_{2}\right)=(1-\alpha) f_{X_{1}, X_{2}}^{a c}\left(x_{1}, x_{2}\right)+\alpha f_{X_{1}, X_{2}}^{s i}\left(\max \left\{x_{1}, x_{2}\right\}\right) \mathcal{I}_{\left(x_{1}=x_{2}\right)},
$$


where $\mathcal{I}_{(.)}$is the indicator function and

$$
(1-\alpha) f_{X_{1}, X_{2}}^{a c}\left(x_{1}, x_{2}\right)=\frac{\partial^{2}}{\partial x_{1} \partial x_{2}} S_{X_{1}, X_{2}}\left(x_{1}, x_{2}\right) .
$$

Following Bemis et al. (1972), the function $f_{X_{1}, X_{2}}\left(x_{1}, x_{2}\right)$ is a density function relative to the measure $\mu$, where $\mu(A)=\gamma_{2}(A)+\gamma_{1}\left(\left(A \cap\left\{x_{1}=x_{2}\right\}\right)_{p}\right)$, where $\gamma_{i}$ denotes the $i$-dimensional Lebesgue measure and the subscript $p$ denotes the projection of the set in question onto the $x_{1}$ axis.

To ensure existence of $S_{X_{1}, X_{2}}\left(x_{1}, x_{2}\right)$ we should evaluate the parameter $\alpha$ which is equivalent to impose that $1-\alpha=\mathbb{P}\left(X_{1}>X_{2}\right)+\mathbb{P}\left(X_{2}>X_{1}\right) \in[0,1]$, so one has to calculate the probabilities in the last sum. We have

$$
\mathbb{P}\left(X_{1}>X_{2}\right)=\int_{0}^{\infty} \int_{0}^{u}(1-\alpha) f_{X_{1}, X_{2}}^{a c}(u, v) \mathrm{d} v \mathrm{~d} u
$$

Computing the inner integral $I(u)=\int_{0}^{u}(1-\alpha) f_{X_{1}, X_{2}}^{a c}(u, v) \mathrm{d} v$ we get

$$
I(u)=-f_{X_{1}}(0) \exp \left\{-a_{0} u-\frac{a_{2}+a_{1}}{2} u^{2}\right\}-a_{1} u \exp \left\{-a_{0} u-\frac{a_{2}+a_{1}}{2} u^{2}\right\}+f_{X_{1}}(u) .
$$

Therefore,

$$
\begin{aligned}
\mathbb{P}\left(X_{1}>X_{2}\right) & =\int_{0}^{\infty} I(u) \mathrm{d} u \\
& =-f_{X_{1}}(0) \int_{0}^{\infty} \exp \left\{-a_{0} u-\frac{a_{2}+a_{1}}{2} u^{2}\right\} \mathrm{d} u \\
& -a_{1} \int_{0}^{\infty} u \exp \left\{-a_{0} u-\frac{a_{2}+a_{1}}{2} u^{2}\right\} \mathrm{d} u+1 .
\end{aligned}
$$

In order to solve integrals in (5.14), we use the following two expressions taken from Gradshteyn and Ryzhik (2007) for positive constants $c$ and $d$ :

$$
\int_{0}^{\infty} \exp \left\{-c u^{2}-d u\right\} \mathrm{d} u=\frac{1}{2} \sqrt{\frac{\pi}{c}} \exp \left\{\frac{d^{2}}{4 c}\right\}\left[1-\operatorname{Erf}\left(\frac{d}{2 \sqrt{c}}\right)\right],
$$

(see equation 3.322.2 on page 336), and

$$
\int_{0}^{\infty} u \exp \left\{-c u^{2}-d u\right\} \mathrm{d} u=\frac{1}{2 c}-\frac{d}{4 c} \sqrt{\frac{\pi}{c}} \exp \left\{\frac{d^{2}}{4 c}\right\}\left[1-\operatorname{Erf}\left(\frac{d}{2 \sqrt{c}}\right)\right],
$$

(see equation 3.462.5 on page 365), where $\operatorname{Er} f(x)=\frac{2}{\sqrt{\pi}} \int_{0}^{x} \exp \left\{-t^{2}\right\} \mathrm{d} t$. 
Adjusting the constants $c=\frac{a_{1}+a_{2}}{2}$ and $d=a_{0}$ we obtain from (5.14)

$$
\begin{aligned}
\mathbb{P}\left(X_{1}>X_{2}\right) & =1-f_{X_{1}}(0) \sqrt{\frac{\pi}{2\left(a_{1}+a_{2}\right)}} \exp \left\{\frac{a_{0}^{2}}{2\left(a_{1}+a_{2}\right)}\right\}\left[1-\operatorname{Erf}\left(\frac{a_{0}}{\sqrt{2\left(a_{1}+a_{2}\right)}}\right)\right] \\
& -\frac{a_{1}}{a_{1}+a_{2}}+\frac{a_{0} a_{1}}{a_{1}+a_{2}} \sqrt{\frac{\pi}{2\left(a_{1}+a_{2}\right)}} \exp \left\{\frac{a_{0}^{2}}{2\left(a_{1}+a_{2}\right)}\right\}\left[1-\operatorname{Erf}\left(\frac{a_{0}}{\sqrt{2\left(a_{1}+a_{2}\right)}}\right)\right],
\end{aligned}
$$

i.e. $\mathbb{P}\left(X_{1}>X_{2}\right)=\frac{a_{2}}{a_{1}+a_{2}}-\left[f_{X_{1}}(0)-\frac{a_{0} a_{1}}{a_{1}+a_{2}}\right] \sqrt{\frac{\pi}{2\left(a_{1}+a_{2}\right)}} \exp \left\{\frac{a_{0}^{2}}{2\left(a_{1}+a_{2}\right)}\right\}\left[1-\operatorname{Erf}\left(\frac{a_{0}}{\sqrt{2\left(a_{1}+a_{2}\right)}}\right)\right]$.

In a similar way we get

$$
\mathbb{P}\left(X_{2}>X_{1}\right)=\frac{a_{1}}{a_{1}+a_{2}}-\left[f_{X_{2}}(0)-\frac{a_{0} a_{2}}{a_{1}+a_{2}}\right] \sqrt{\frac{\pi}{2\left(a_{1}+a_{2}\right)}} e^{\frac{a_{0}^{2}}{2\left(a_{1}+a_{2}\right)}}\left[1-\operatorname{Erf}\left(\frac{a_{0}}{\sqrt{2\left(a_{1}+a_{2}\right)}}\right)\right] .
$$

Since $\alpha=\mathbb{P}\left(X_{1}=X_{2}\right)=1-\mathbb{P}\left(X_{1}>X_{2}\right)-\mathbb{P}\left(X_{2}>X_{1}\right)$, from the last expressions and (5.14) we finally arrive to

$$
\alpha=\left[f_{X_{1}}(0)+f_{X_{2}}(0)-a_{0}\right] \sqrt{\frac{\pi}{2\left(a_{1}+a_{2}\right)}} \exp \left\{\frac{a_{0}^{2}}{2\left(a_{1}+a_{2}\right)}\right\}\left[1-\operatorname{Erf}\left(\frac{a_{0}}{\sqrt{2\left(a_{1}+a_{2}\right)}}\right)\right] .
$$

But $\alpha \in[0,1]$ and we obtain from (5.15) the permissible values of the constants: $a_{0} \geq 0, a_{1} \geq 0$ and $a_{2} \geq 0$, with $a_{1}+a_{2}>0$. So, we got relation (5.12) and the theorem is proved.

Proposition 5.2.15. The singular component of $S_{X_{1}, X_{2}}\left(x_{1}, x_{2}\right)$ in (5.6) is given by

$$
S_{X_{1}, X_{2}}^{s i}\left(x_{1}, x_{2}\right)=\frac{1-\operatorname{Erf}\left(\frac{a_{0}+\left(a_{1}+a_{2}\right) \max \left(x_{1}, x_{2}\right)}{\sqrt{2\left(a_{1}+a_{2}\right)}}\right)}{1-\operatorname{Erf}\left(\frac{a_{0}}{\sqrt{2\left(a_{1}+a_{2}\right)}}\right)} .
$$

Proof. Let $x_{1}=x_{2}=x \geq 0$ in (2.11) to obtain

$$
\alpha S_{X_{1}, X_{2}}^{s i}(x, x)=S_{X_{1}, X_{2}}(x, x)-(1-\alpha) S_{X_{1}, X_{2}}^{a c}(x, x) .
$$

In order to evaluate $(1-\alpha) S_{X_{1}, X_{2}}^{a c}(x, x)$ in (5.16) we proceed with the following decomposition

$$
\begin{aligned}
(1-\alpha) S_{X_{1}, X_{2}}^{a c}(x, x) & =\mathbb{P}\left(X_{1}>X_{2} \geq x\right)+\mathbb{P}\left(X_{2}>X_{1} \geq x\right) \\
& =\int_{x}^{\infty} \int_{x}^{u}(1-\alpha) f_{X_{1}, X_{2}}^{a c}(u, v) \mathrm{d} v \mathrm{~d} u+\int_{x}^{\infty} \int_{x}^{v}(1-\alpha) f_{X_{1}, X_{2}}^{a c}(u, v) \mathrm{d} u \mathrm{~d} v .
\end{aligned}
$$

Denoting by $I_{i}(x), i=1,2$, each one of the integrals in (5.17), let us first compute

$$
I_{1}(x)=\int_{x}^{\infty} \int_{x}^{u}(1-\alpha) f_{X_{1}, X_{2}}^{a c}(u, v) \mathrm{d} v \mathrm{~d} u,
$$

where $(1-\alpha) f_{X_{1}, X_{2}}^{a c}\left(x_{1}, x_{2}\right)$ is obtained from relations (5.6) and (5.13). The inner integral in previous 
expression is given by

$$
\begin{aligned}
\int_{x}^{u}(1-\alpha) f_{X_{1}, X_{2}}^{a c}(u, v) \mathrm{d} v & =f_{X_{1}}(u-x) \exp \left\{-a_{0} x-a_{1} u x-\frac{a_{2}-a_{1}}{2} x^{2}\right\} \\
& +a_{1} x S_{X_{1}}(u-x) \exp \left\{-a_{0} x-a_{1} u x-\frac{a_{2}-a_{1}}{2} x^{2}\right\} \\
& -f_{X_{1}}(0) \exp \left\{-a_{0} u-\frac{a_{2}+a_{1}}{2} u^{2}\right\}-a_{1} u \exp \left\{-a_{0} u-\frac{a_{2}+a_{1}}{2} u^{2}\right\} .
\end{aligned}
$$

In the last relation one can recognize that the two first terms in the right hand side of the equality can be written as

$$
\begin{aligned}
& f_{X_{1}}(u-x) \exp \left\{-a_{0} x-a_{1} u x-\frac{a_{2}-a_{1}}{2} x^{2}\right\}+a_{1} x S_{X_{1}}(u-x) \exp \left\{-a_{0} x-a_{1} u x-\frac{a_{2}-a_{1}}{2} x^{2}\right\} \\
& =-\frac{d}{d u} S_{X_{1}}(u-x) \exp \left\{-a_{0} x-a_{1} u x-\frac{a_{2}-a_{1}}{2} x^{2}\right\},
\end{aligned}
$$

so that their integral is given by

$$
\begin{aligned}
\int_{x}^{\infty}\left\{-\frac{d}{d u} S_{X_{1}}(u-x) \exp \left\{-a_{0} x-a_{1} u x-\frac{a_{2}-a_{1}}{2} x^{2}\right\}\right\} \mathrm{d} u & =\exp \left\{-a_{0} x-\frac{a_{1}+a_{2}}{2} x^{2}\right\} \\
& =S_{X_{1}, X_{2}}(x, x) .
\end{aligned}
$$

Now, in order to finish the evaluation of $I_{1}(x)$, two integrals remain to be calculated. They are respectively given by

$$
\begin{aligned}
\int_{x}^{\infty}\left\{-f_{X_{1}}(0) \exp \left\{-a_{0} u-\frac{a_{2}+a_{1}}{2} u^{2}\right\}\right\} \mathrm{d} u & =-f_{X_{1}}(0) \sqrt{\frac{\pi}{2\left(a_{1}+a_{2}\right)}} \\
& \times \exp \left\{\frac{a_{0}^{2}}{2\left(a_{1}+a_{2}\right)}\right\}\left[1-\operatorname{Erf}\left(\frac{a_{0}+\left(a_{1}+a_{2}\right) x}{\sqrt{2\left(a_{1}+a_{2}\right)}}\right)\right],
\end{aligned}
$$

and

$$
\begin{aligned}
\int_{x}^{\infty}\left\{-a_{1} u \exp \left\{-a_{0} u-\frac{a_{2}+a_{1}}{2} u^{2}\right\}\right\} \mathrm{d} u & =-\frac{a_{1} \exp \left\{-a_{0} x-\frac{a_{1}+a_{2}}{2} x^{2}\right\}}{a_{1}+a_{2}} \\
& +\frac{a_{0} a_{1}}{a_{1}+a_{2}} \sqrt{\frac{\pi}{2\left(a_{1}+a_{2}\right)}} \exp \left\{\frac{a_{0}^{2}}{2\left(a_{1}+a_{2}\right)}\right\} \\
& \times\left[1-\operatorname{Erf}\left(\frac{a_{0}+\left(a_{1}+a_{2}\right) x}{\sqrt{2\left(a_{1}+a_{2}\right)}}\right)\right] .
\end{aligned}
$$


Therefore,

$$
\begin{aligned}
I_{1}(x) & =S_{X_{1}, X_{2}}(x, x)-f_{X_{1}}(0) \sqrt{\frac{\pi}{2\left(a_{1}+a_{2}\right)}} \exp \left\{\frac{a_{0}^{2}}{2\left(a_{1}+a_{2}\right)}\right\}\left[1-\operatorname{Erf}\left(\frac{a_{0}+\left(a_{1}+a_{2}\right) x}{\sqrt{2\left(a_{1}+a_{2}\right)}}\right)\right] \\
& -\frac{a_{1}}{a_{1}+a_{2}} S_{X_{1}, X_{2}}(x, x)+\frac{a_{0} a_{1}}{a_{1}+a_{2}} \sqrt{\frac{\pi}{2\left(a_{1}+a_{2}\right)}} \exp \left\{\frac{a_{0}^{2}}{2\left(a_{1}+a_{2}\right)}\right\}\left[1-\operatorname{Erf}\left(\frac{a_{0}+\left(a_{1}+a_{2}\right) x}{\sqrt{2\left(a_{1}+a_{2}\right)}}\right)\right] \\
& =S_{X_{1}, X_{2}}(x, x)\left(1-\frac{a_{1}}{a_{1}+a_{2}}\right) \\
& -\left(f_{X_{1}}(0)-\frac{a_{0} a_{1}}{a_{1}+a_{2}}\right) \sqrt{\frac{\pi}{2\left(a_{1}+a_{2}\right)}} \exp \left\{\frac{a_{0}^{2}}{2\left(a_{1}+a_{2}\right)}\right\}\left[1-\operatorname{Erf}\left(\frac{a_{0}+\left(a_{1}+a_{2}\right) x}{\sqrt{2\left(a_{1}+a_{2}\right)}}\right)\right] .
\end{aligned}
$$

Analogously,

$$
\begin{aligned}
I_{2}(x) & =S_{X_{1}, X_{2}}(x, x)\left(1-\frac{a_{2}}{a_{1}+a_{2}}\right) \\
& -\left(f_{X_{2}}(0)-\frac{a_{0} a_{2}}{a_{1}+a_{2}}\right) \sqrt{\frac{\pi}{2\left(a_{1}+a_{2}\right)}} \exp \left\{\frac{a_{0}^{2}}{2\left(a_{1}+a_{2}\right)}\right\}\left[1-\operatorname{Erf}\left(\frac{a_{0}+\left(a_{1}+a_{2}\right) x}{\sqrt{2\left(a_{1}+a_{2}\right)}}\right)\right] .
\end{aligned}
$$

Thus we have

$$
\begin{aligned}
(1-\alpha) S_{X_{1}, X_{2}}^{a c}(x, x) & =S_{X_{1}, X_{2}}(x, x) \\
& -\left(f_{X_{1}}(0)+f_{X_{2}}(0)-a_{0}\right) \sqrt{\frac{\pi}{2\left(a_{1}+a_{2}\right)}} \exp \left\{\frac{a_{0}^{2}}{2\left(a_{1}+a_{2}\right)}\right\} \\
& \times\left[1-\operatorname{Erf}\left(\frac{a_{0}+\left(a_{1}+a_{2}\right) x}{\sqrt{2\left(a_{1}+a_{2}\right)}}\right)\right] .
\end{aligned}
$$

Thus, substituting (5.15) and (5.18) in (5.16)

$$
S_{X_{1}, X_{2}}^{s i}(x, x)=\frac{1-\operatorname{Erf}\left(\frac{a_{0}+\left(a_{1}+a_{2}\right) x}{\sqrt{2\left(a_{1}+a_{2}\right)}}\right)}{1-\operatorname{Erf}\left(\frac{a_{0}}{\sqrt{2\left(a_{1}+a_{2}\right)}}\right)} .
$$

To finish the proof, when $x_{1} \neq x_{2}$ since $S_{X_{1}, X_{2}}^{s i}\left(x_{1}, x_{2}\right)$ is concentrated on $x_{1}=x_{2}=x \geq 0$ we substitute $x$ by $\max \left(x_{1}, x_{2}\right)$ on the previous expression.

Remark 5.2.16. When $a_{1}=a_{2}=0$, Marshall and Olkin (1967) obtained the distribution of the singular component as

$$
S_{X_{1}, X_{2}}^{s i}\left(x_{1}, x_{2}\right)=\exp \left\{-a_{0} \max \left(x_{1}, x_{2}\right)\right\},
$$

see their equation (5.6).

Notice that if inequality (5.11) is fulfilled for some $a_{0}=u>0$, then it is also satisfied for all $a_{0} \geq u$. Denote by

$$
\tau=\text { the greatest lower bound of the set of possible values of } a_{0} \text { satisfying (5.11). }
$$

Depending on the marginal densities, it may happen in some special cases that $\tau>f_{X_{1}}(0)+f_{X_{2}}(0)$, 
which contradicts (5.12) since always $\alpha=\mathbb{P}\left(X_{1}=X_{2}\right) \geq 0$, i.e. $a_{0} \leq f_{X_{1}}(0)+f_{X_{2}}(0)$ according to (5.15). Such high $\tau$-values are unacceptable for validation of our model.

The range of possible values of $a_{0}$ are shown in Proposition 5.2.17 which is crucial for the construction of proper bivariate survival functions belonging to the class $\mathcal{L}(\mathbf{x} ; \mathbf{a})$ from specified marginal densities. In Section 5.3.2 we provide related examples.

Proposition 5.2.17. Suppose $\tau \leq f_{X_{1}}(0)+f_{X_{2}}(0)$. If

$$
a_{0} \in\left[\max \left\{\tau, \max \left(f_{X_{1}}(0), f_{X_{2}}(0)\right)\right\}, f_{X_{1}}(0)+f_{X_{2}}(0)\right],
$$

then Theorem 5.2.14 is verified.

Proof. In the absence of singularity (whenever $\alpha=\mathbb{P}\left(X_{1}=X_{2}\right)=0$ ), one concludes from (5.15) that $f_{X_{1}}(0)+f_{X_{2}}(0)-a_{0}=0$. Therefore, always $a_{0} \leq f_{X_{1}}(0)+f_{X_{2}}(0)$, which is the upper bound for $a_{0}$ in (5.19).

The increase of the singular contribution into $S_{X_{1}, X_{2}}\left(x_{1}, x_{2}\right)$ implies increasing of the nonnegative parameter $\alpha$ up to 1 . Let us denote by

$$
E\left(a_{0}, a_{1}, a_{2}\right)=a_{0} \sqrt{\frac{\pi}{2\left(a_{1}+a_{2}\right)}} \exp \left\{\frac{a_{0}^{2}}{2\left(a_{1}+a_{2}\right)}\right\}\left[1-\operatorname{Erf}\left(\frac{a_{0}}{\sqrt{2\left(a_{1}+a_{2}\right)}}\right)\right] .
$$

It is direct to check that $0 \leq E\left(a_{0}, a_{1}, a_{2}\right) \leq 1$. We may represent (5.14) as

$$
\mathbb{P}\left(X_{1}>X_{2}\right)=1-\frac{f_{X_{1}}(0)}{a_{0}} E\left(a_{0}, a_{1}, a_{2}\right)-\frac{a_{1}}{a_{1}+a_{2}}\left[1-E\left(a_{0}, a_{1}, a_{2}\right)\right] .
$$

The right hand side of the last equation is nonnegative if $f_{X_{1}}(0) \leq a_{0}$. By analogy, from the expression for $\mathbb{P}\left(X_{2}>X_{1}\right)$ we obtain $f_{X_{2}}(0) \leq a_{0}$.

Notice that if $a_{0} \in\left[\max \left\{f_{X_{1}}(0), f_{X_{2}}(0)\right\}, f_{X_{1}}(0)+f_{X_{2}}(0)\right]$ then $\alpha=\mathbb{P}\left(X_{1}=X_{2}\right) \in[0,1]$. Finally, the lower bound in (5.19) can be obtained by taking into account the restriction on $a_{0}$ imposed by inequality (5.11) and possible related $\tau$-values. This completes the proof.

Remark 5.2.18 (Absolutely continuous rule). Observe that whenever the upper bound $a_{U}$ for $a_{0}$ given by (5.19) is attainable, i.e. if $a_{U}=a_{0}=f_{X_{1}}(0)+f_{X_{2}}(0)$, one obtains an absolutely continuous bivariate distribution that belongs to the class $\mathcal{L}(\mathbf{x} ; \mathbf{a})$, i.e., for which (5.2) holds true. Therefore, equation (5.15), besides representing the constraint $a_{1}+a_{2}>0$, also offers a way to identify the presence of singularity.

Consider a joint distribution given by $S_{X_{1}, X_{2}}\left(x_{1}, x_{2}\right)=\exp \left\{-0.5 a_{1} x_{1}^{2}-0.5 a_{2} x_{2}^{2}\right\}$. Observe that both $f_{X_{1}}(0)=f_{X_{2}}(0)=0$ implying $a_{0}=0$ and $\alpha=\mathbb{P}\left(X_{1}=X_{2}\right)=0$. This special absolutely continuous bivariate distribution with independent marginals case may be treated as an "exception", see the paragraph following Corollary 5.3.1.

Hence, for absolutely continuous distributions belonging to the class $\mathcal{L}(\mathbf{x} ; \mathbf{a})$, it may happen that the lower and upper bound in (5.19) coincide, being even zero when $f_{X_{1}}(0)=f_{X_{2}}(0)=0$, or equivalently, when $r_{X_{1}}(0)=r_{X_{2}}(0)=0$, indicating that $a_{0}=0$.

Remark 5.2.19 (Singularity of the GMO models). Let us consider the joint survival function of $\left(Y_{1}, Y_{2}\right)$ belonging to the class of GMO distributions and represented by (5.5), with $\lambda_{i}=\frac{a_{i}}{2}, i=1,2$, 
and $\lambda_{3}=a_{0}$. It is direct to check that

$$
\mathbb{P}\left(Y_{1}=Y_{2}\right)=a_{0} \sqrt{\frac{\pi}{2\left(a_{1}+a_{2}\right)}} \exp \left\{\frac{a_{0}^{2}}{2\left(a_{1}+a_{2}\right)}\right\}\left[1-\operatorname{Erf}\left(\frac{a_{0}}{\sqrt{2\left(a_{1}+a_{2}\right)}}\right)\right] .
$$

Observe that the right hand side in the last equation is just the function $E\left(a_{0}, a_{1}, a_{2}\right)$ used in the proof of Proposition 5.2.17.

Remark 5.2.20 (Singularity of the $B L M P_{1}$ models). In the particular case $a_{1}=a_{2}=0$, the condition (5.11) transforms into inequality (ii) of Theorem 5.1 in Marshall and Olkin (1967). In addition, the possible interval values of $a_{0}$ in (5.19) are compatible with those given by Kulkarni (2006) in her Remark 5.2.12.

\subsubsection{Geometric interpretations}

Here we will illustrate geometrically the properties of the sum $r\left(x_{1}, x_{2}\right)$ specified by relation by $(5.2)$.

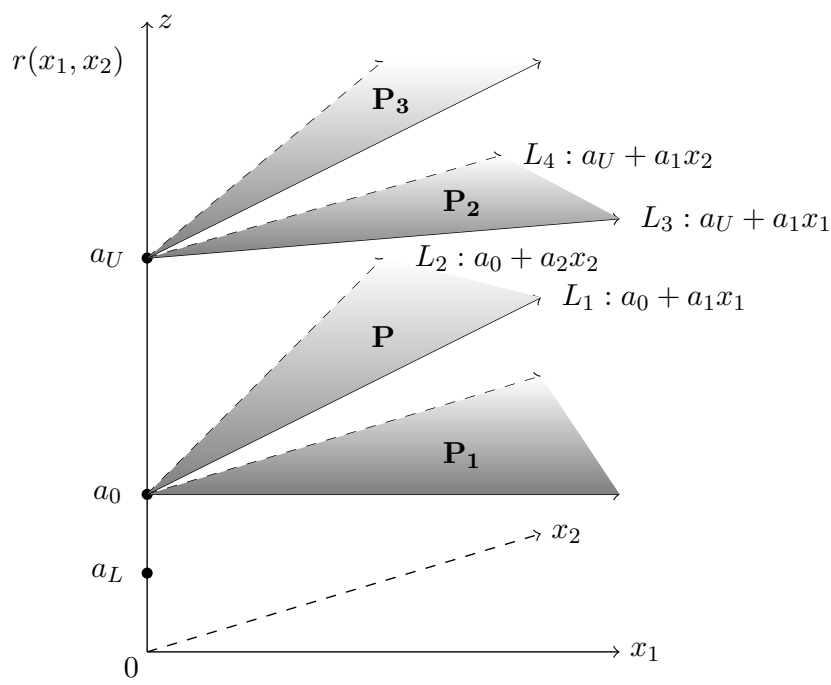

Figure 5.2: $\quad$ Geometric representation of the class $\mathcal{L}(\mathbf{x} ; \mathbf{a})$

Graphical interpretations for the distributions belonging to the class $\mathcal{L}(\mathbf{x} ; \mathbf{a})$ are shown in Figure 5.2. The vertical axis $0 z$ represents the values of the sum $r\left(x_{1}, x_{2}\right)$ of the hazard vector elements, i.e. $r_{1}\left(x_{1}, x_{2}\right)+r_{2}\left(x_{1}, x_{2}\right)$. One can localize the lower and upper bounds $a_{L}$ and $a_{U}$ of the constant $a_{0}$ according to (5.19), i.e.

$$
a_{L}=\max \left\{\tau, \max \left(f_{X_{1}}(0), f_{X_{2}}(0)\right)\right\} \quad \text { and } \quad a_{U}=f_{X_{1}}(0)+f_{X_{2}}(0) .
$$

In general, all members of the class $\mathcal{L}(\mathbf{x} ; \mathbf{a})$ lie in the first octant over the plane $\{P\}$ given analytically by $P: r\left(x_{1}, x_{2}\right)=a_{0}+a_{1} x_{1}+a_{2} x_{2}$. The plane $\{P\}$ crosses the axis $0 z$ at the point $\left(0,0, a_{0}\right)$ and crosses the planes $\left\{x_{2}=0\right\}$ and $\left\{x_{1}=0\right\}$ in lines $L_{1}: a_{0}+a_{1} x_{1}$ and $L_{2}: a_{0}+a_{2} x_{2}$, respectively. The lines $\left\{L_{1}\right\}$ and $\left\{L_{2}\right\}$ have always nonnegative inclination, since $a_{1}, a_{2} \geq 0$.

One can recognize the following basic cases displayed on Figure 5.2.

(i) The plane $P_{1}: r\left(x_{1}, x_{2}\right)=a_{0}$ crosses $0 z$ at the point $\left(0,0, a_{0}\right)$ and is parallel to the plane 
$\{z=0\}$. The plane $\left\{P_{1}\right\}$ represents distributions possessing BLM $P_{1}$ and exhibiting singularity along the line $x_{1}=x_{2}=x \geq 0$;

(ii) The plane $P_{2}: r\left(x_{1}, x_{2}\right)=a_{U}+a_{1} x_{1}+a_{1} x_{2}$ is the image of $B L M P_{2}$ (characterizing the Gumbel's bivariate exponential distribution from (5.3)). The plane $\left\{P_{2}\right\}$ crosses the planes $\left\{x_{2}=0\right\}$ and $\left\{x_{1}=0\right\}$ in the lines $L_{3}: a_{U}+a_{1} x_{1}$ and $L_{4}: a_{U}+a_{1} x_{2}$, with equal inclination. Observe that the image of all exchangeable absolutely continuous distributions belonging to the class $\mathcal{L}(\mathbf{x} ; \mathbf{a})$ have similar characteristics;

(iii) The planes representing the absolutely continuous bivariate distributions as well as those with independent marginals cross the axis $0 z$ at the point $\left(0,0, a_{U}\right)$. The corresponding common lines with planes $\left\{x_{2}=0\right\}$ and $\left\{x_{1}=0\right\}$ have nonnegative inclination which may be symmetric (if $a_{1}=a_{2}$ ), asymmetric (when $a_{1} \neq a_{2}$ ), or parallel to the plane $\{z=0\}$, whenever $a_{1}=$ $a_{2}=0$. The plane $P_{3}: r\left(x_{1}, x_{2}\right)=a_{U}+a_{1} x_{1}+a_{2} x_{2}$ is a typical example.

\subsection{Construction of bivariate distributions belonging to the class $\mathcal{L}(\mathbf{x} ; \mathbf{a})$}

In this section we will offer procedures to build bivariate distributions belonging to the class $\mathcal{L}(\mathbf{x} ; \mathbf{a})$ for the following basic situations: those having independent marginals; under the knowledge of marginal failure rates and finally, by using some known $\mathcal{L}(\mathbf{x} ; \mathbf{a})$-distribution as a baseline. The methodology is supported by examples.

\subsubsection{Bivariate distributions with independent marginals}

Let the random variables $X_{1}$ and $X_{2}$ be independent. Hence

$$
S_{X_{1}, X_{2}}\left(x_{1}, x_{2}\right)=S_{X_{1}}\left(x_{1}\right) S_{X_{2}}\left(x_{2}\right) \text { for all } x_{1}, x_{2} \geq 0
$$

In terms of conditional failure rates, the independence between $X_{1}$ and $X_{2}$ implies that $r_{i}\left(x_{1}, x_{2}\right)=$ $r_{X_{i}}\left(x_{i}\right), i=1,2$. Therefore, relation (5.2) transforms into

$$
r\left(x_{1}, x_{2}\right)=r_{X_{1}}\left(x_{1}\right)+r_{X_{2}}\left(x_{2}\right)=a_{0}+a_{1} x_{1}+a_{2} x_{2} .
$$

The last equation is equivalent to both

$$
r_{X_{1}}\left(x_{1}\right)=\alpha_{1}+a_{1} x_{1} \quad \text { and } \quad r_{X_{2}}\left(x_{2}\right)=\alpha_{2}+a_{2} x_{2},
$$

where $\alpha_{1} \in\left[0, a_{0}\right]$ and $\alpha_{2}=a_{0}-\alpha_{1}$. This tells us that $r_{X_{1}}(0)+r_{X_{2}}(0)=a_{0}$, i.e. $S_{X_{1}, X_{2}}\left(x_{1}, x_{2}\right)$ should be absolutely continuous, according to Remark 5.2.18.

Hence, we obtain the following result.

Corollary 5.3.1. The class $\mathcal{L}(\mathbf{x} ; \mathbf{a})$ has independent marginals if and only if their marginal survival functions $S_{X_{i}}\left(x_{i}\right)$ have one of the following three possible analytic forms

$$
(i * 1) \exp \left\{-\alpha_{i} x_{i}\right\}, \quad(i * 2) \exp \left\{-0.5 a_{i} x_{i}^{2}\right\} \quad \text { and } \quad(i * 3) \exp \left\{-\alpha_{i} x_{i}-0.5 a_{i} x_{i}^{2}\right\}, i=1,2,
$$


Table 5.1: Basic expressions for independence case

\begin{tabular}{|c|c|c|c|}
\hline$S_{X_{1}}\left(x_{1}\right)$ & $S_{X_{2}}\left(x_{2}\right)$ & $S_{X_{1}, X_{2}}\left(x_{1}, x_{2}\right)=S_{X_{1}}\left(x_{1}\right) S_{X_{2}}\left(x_{2}\right)$ & $r_{X_{1}}\left(x_{1}\right)+r_{X_{2}}\left(x_{2}\right)$ \\
\hline$(1 * 1)$ & $(2 * 1)$ & $\exp \left\{-\alpha_{1} x_{2}-\alpha_{2} x_{2}\right\}$ & $a_{0}$ \\
\hline$(1 * 1)$ & $(2 * 2)$ & $\exp \left\{-\alpha_{1} x_{1}-0.5 a_{2} x_{2}^{2}\right\}$ & $\alpha_{1}+a_{2} x_{2}$ \\
\hline$(1 * 1)$ & $(2 * 3)$ & $\exp \left\{-\alpha_{1} x_{1}-\alpha_{2} x_{2}-0.5 a_{2} x_{2}^{2}\right\}$ & $a_{0}+a_{2} x_{2}$ \\
\hline$(1 * 2)$ & $(2 * 3)$ & $\exp \left\{-0.5 a_{1} x_{1}^{2}-\alpha_{2} x_{2}-0.5 a_{2} x_{2}^{2}\right\}$ & $\alpha_{2}+a_{1} x_{1}+a_{2} x_{2}$ \\
\hline$(1 * 3)$ & $(2 * 3)$ & $\exp \left\{-\alpha_{1} x_{1}-0.5 a_{1} x_{1}^{2}-\alpha_{2} x_{2}-0.5 a_{2} x_{2}^{2}\right\}$ & $a_{0}+a_{1} x_{1}+a_{2} x_{2}$ \\
\hline
\end{tabular}

where $\alpha_{1}+\alpha_{2}=a_{0}$ and $x_{1}, x_{2} \geq 0$.

The marginal distributions shown in Corollary 5.3.1 generate 9 admissible joint distributions belonging to the class $\mathcal{L}(\mathbf{x} ; \mathbf{a})$. Note that one would obtain joint distribution with $a_{0}=0$ if the marginals are of the type $(1 * 2)$ and $(2 * 2)$.

We present in Table 5.1 basic expressions for joint survival function and corresponding sum of hazard elements in independent case. Because of the symmetry in the corresponding expressions, we did not include in the Table 5.1 the cases when $r\left(x_{1}, x_{2}\right)$ is equal to $\alpha_{2}+a_{1} x_{1}, a_{0}+a_{1} x_{1}$ and $\alpha_{1}+a_{1} x_{1}+a_{2} x_{2}$.

Geometrical interpretation of the sum $r_{1}\left(x_{1}, x_{2}\right)+r_{2}\left(x_{1}, x_{2}\right)$ in independence case can be identified by Plane $\left\{P_{3}\right\}$ in Figure 5.2, see the related discussion in item (iii), Section 5.2.4.

\subsubsection{Bivariate distributions with given marginal failure rates}

As we noted in the proof of Theorem 5.2.14, if the survival function $S_{X_{1}, X_{2}}\left(x_{1}, x_{2}\right)$ given by (5.6) is proper then $\frac{\partial^{2}}{\partial x_{1} \partial x_{2}} S_{X_{1}, X_{2}}\left(x_{1}, x_{2}\right)$ should be nonnegative. This condition is equivalent to the requirement

$$
S_{X_{1}, X_{2}}\left(x_{1}, y_{1}\right)+S_{X_{1}, X_{2}}\left(x_{2}, y_{2}\right)-S_{X_{1}, X_{2}}\left(x_{1}, y_{2}\right)-S_{X_{1}, X_{2}}\left(x_{2}, y_{1}\right) \geq 0
$$

for any two points $\left(x_{1}, y_{1}\right)$ and $\left(x_{2}, y_{2}\right)$ in $\mathbf{R}_{+}^{2}$ such that $x_{1} \leq x_{2}$ and $y_{1} \leq y_{2}$. For example if $x_{1} \leq x_{2} \leq y_{1} \leq y_{2}$ and $a_{2}=0$ we conclude from (5.6) that the last inequality is equivalent to

$$
\frac{S_{X_{2}}\left(y_{1}-x_{1}\right)-S_{X_{2}}\left(y_{2}-x_{1}\right)}{S_{X_{2}}\left(y_{1}-x_{2}\right)-S_{X_{2}}\left(y_{2}-x_{2}\right)} \leq \exp \left\{-\left(x_{2}-x_{1}\right)\left[a_{0}+\frac{a_{1}}{2}\left(x_{2}+x_{1}\right)\right]\right\} .
$$

In general, such constraints between marginal survival functions are not easily verified. Relations (5.11) and (5.12) in Theorem 5.2.14 give alternative conditions in terms of absolutely continuous marginal densities $f_{X_{i}}(x), i=1,2$. Depending on the complexity of the analytical form of the densities involved, these restrictions may be also difficult to check.

\section{Alternative marginal failure rates restrictions}

The next result gives another set of equivalent constraints for the model parameters but in terms of marginal failure rates $r_{X_{i}}(x)$. This may serve as a useful guide for constructing bivariate distributions possessing property (5.2), i.e. belonging to the class $\mathcal{L}(\mathbf{x} ; \mathbf{a})$.

Theorem 5.3.2. Let the marginal failure rates $r_{X_{i}}(x), i=1,2$, be differentiable functions and for 
some nonnegative constants $a_{0}, a_{1}$ and $a_{2}$, with $a_{1}+a_{2}>0$, the following relations hold

$$
\begin{gathered}
0 \leq r_{X_{i}}\left(x_{i}\right) \leq a_{0}+a_{i} x_{i} \\
r_{X_{i}}\left(x_{i}-x_{j}\right)\left[K\left(x_{i}, x_{j}\right)-a_{i} x_{j}-r_{X_{i}}\left(x_{i}-x_{j}\right)\right] \\
+\frac{d}{d x_{i}} r_{X_{i}}\left(x_{i}-x_{j}\right)+a_{i}\left[x_{j} K\left(x_{i}, x_{j}\right)-1\right] \geq 0,
\end{gathered}
$$

with $K\left(x_{i}, x_{j}\right)=a_{0}+a_{i} x_{i}+\left(a_{j}-a_{i}\right) x_{j}$, for $x_{i} \geq x_{j} \geq 0, i, j=1,2, i \neq j$;

$$
a_{0} \in\left[\max \left\{\tau, \max \left(r_{X_{1}}(0), r_{X_{2}}(0)\right)\right\}, r_{X_{1}}(0)+r_{X_{2}}(0)\right]
$$

where $\tau$ denotes the greatest lower bound of the set of values of $a_{0}$ for which the inequality (5.20b) is satisfied.

Then the joint survival function $S_{X_{1}, X_{2}}\left(x_{1}, x_{2}\right)$ given by (5.6) is proper with marginals

$$
S_{X_{i}}\left(x_{i}\right)=\exp \left(-\int_{0}^{x_{i}} r_{X_{i}}(u) \mathrm{d} u\right)
$$

$x_{i} \geq 0, i=1,2$. The joint distribution is absolutely continuous if $a_{0}=r_{X_{1}}(0)+r_{X_{2}}(0)$, otherwise it possesses a singular component.

Proof. Let the univariate failure rates $r_{X_{i}}\left(x_{i}\right), i=1,2$ be differentiable functions. Then $S_{X_{1}, X_{2}}\left(x_{1}, x_{2}\right)$ given by (5.6) is a valid survival function with components of the hazard gradient vector satisfying (5.2), when (5.20a) to $(5.20 \mathrm{c})$ are fulfilled.

Really, let us consider the case when $x_{2} \geq x_{1} \geq 0$. Then substituting $x_{1}=0$ in (5.9) one gets $r_{1}\left(0, x_{2}\right)=a_{0}+a_{2} x_{2}-r_{X_{2}}\left(x_{2}\right) \geq 0$ and therefore $r_{X_{2}}\left(x_{2}\right) \leq a_{0}+a_{2} x_{2}$. By analogy, from (5.10) we conclude that $r_{X_{1}}\left(x_{1}\right) \leq a_{0}+a_{1} x_{1}$ if $x_{1} \geq x_{2} \geq 0$ and (5.20a) is established.

Note that the inequality (5.20b) is equivalent to (5.11) by using the relation

$$
\frac{d}{d x_{i}}\left[\log f_{X_{i}}\left(x_{i}-x_{j}\right)\right]=\frac{d}{d x_{i}}\left[\log r_{X_{i}}\left(x_{i}-x_{j}\right)\right]-r_{X_{i}}\left(x_{i}-x_{j}\right) \quad \text { for } \quad x_{i} \geq x_{j} .
$$

Implication (5.20c) follows from (5.19) since $r_{X_{i}}(0)=f_{X_{i}}(0)$ for $i=1,2$. This completes the proof.

Conditions (5.20a) to (5.20c) in Theorem 5.3.2 imply several simple practical steps that help to fix the permissible parameter space of the coefficients $a_{0}, a_{1}$ and $a_{2}$. We give them in the next remark.

Remark 5.3.3 (Parameter space of the class $\mathcal{L}(\mathbf{x} ; \mathbf{a})$ ). Inequality (5.20a) says that the bivariate distributions from the class $\mathcal{L}(\mathbf{x} ; \mathbf{a})$ satisfying (5.2) cannot have marginal distributions with failure rates $r_{X_{i}}\left(x_{i}\right)$ above the line $a_{0}+a_{i} x_{i}, i=1,2$. For example, distributions with univariate failure rate of the form $a x_{i}^{2}$, for $a>0$ are unable to meet (5.2).

In addition, observe from (5.20a) that the coefficients $a_{i}$ should be nonnegative, because in the opposite case the line $a_{0}+a_{i} x_{i}$ would cross in some moment the zero-level which is impossible, since always $r_{X_{i}}\left(x_{i}\right) \geq 0$ for $i=1,2$. See related Remark 7.3.5. 
Therefore, the parameter space for the coefficients $a_{0}, a_{1}$ and $a_{2}$ satisfying (5.2) in terms of marginal failure rates is given by

$$
a_{0} \in\left[\max \left\{\tau, \max \left(r_{X_{1}}(0), r_{X_{2}}(0)\right)\right\}, r_{X_{1}}(0)+r_{X_{2}}(0)\right], a_{1} \geq 0, a_{2} \geq 0, a_{1}+a_{2}>0 .
$$

Note that admissible values for coefficients $a_{1}$ and $a_{2}$ may be further restricted as a consequence of inequality (5.206) from Theorem 5.3.2, see Examples 5.3.6 and 5.3.7.

Finally, since $r_{X_{i}}($.$) is a failure rate, then \int_{0}^{\infty} r_{X_{i}}(u) \mathrm{d} u=\infty$ and because of (5.20a) the support of $X_{i}, i=1,2$, cannot be bounded from above, i.e. has to be the entire half line $[0, \infty)$.

It may happen that the joint survival function $S_{X_{1}, X_{2}}\left(x_{1}, x_{2}\right)$ is degenerate (i.e. having degenerate marginal distribution). In this case one can obtain from (5.6) that

$$
S_{X_{1}, X_{2}}^{d e}\left(x_{1}, x_{2}\right)= \begin{cases}\exp \left\{-a_{0} x_{2}-a_{1} x_{1} x_{2}-\frac{a_{2}-a_{1}}{2} x_{2}^{2}\right\}, & \text { if } 0 \leq x_{2}<x_{1}=\text { const }, \\ \exp \left\{-a_{0} x-\frac{a_{1}+a_{2}}{2} x^{2}\right\}, & \text { if } 0 \leq x_{2}=x_{1}=x=\text { const }, \\ \exp \left\{-a_{0} x_{1}-a_{2} x_{1} x_{2}-\frac{a_{1}-a_{2}}{2} x_{1}^{2}\right\}, & \text { if } 0 \leq x_{1}<x_{2}=\text { const. }\end{cases}
$$

Obviously, $S_{X_{1}, X_{2}}^{\text {de }}\left(x_{1}, x_{2}\right)$ does not have differentiable failure rates as required by Theorem 5.3.2.

The converse of Theorem 5.3.2 also holds for non-degenerate distributions and the statement is given below.

Proposition 5.3.4. If $S_{X_{1}, X_{2}}\left(x_{1}, x_{2}\right)$ is non-degenerate bivariate survival function given by equation (5.6) and having differentiable marginal failure rates then it must satisfy conditions (5.20a) to (5.20c) in Theorem 5.3.2.

Proof. Follows step by step the proof of Proposition 1 in Kulkarni (2006).

The rules established in Theorem 5.3.2 for building bivariate distribution in the class $\mathcal{L}(\mathbf{x} ; \mathbf{a})$ may be enriched and supported by additional available information regarding monotone behaviour of marginal failure rates. In fact, our class of bivariate distributions $\mathcal{L}(\mathbf{x} ; \mathbf{a})$ may have arbitrary combination of marginal failure rates: increasing, decreasing, constant, bathtub, etc., implying corresponding restrictions for the parameter space, of course.

\section{Examples}

The next three examples illustrate how Theorem 5.3.2 can be used to construct bivariate distributions from $\mathcal{L}(\mathbf{x} ; \mathbf{a})$ with given marginal failure rates.

Example 5.3.5 (Constant failure rate marginals). Let

$$
S_{X_{i}}(x)=\exp \left\{-\lambda_{i} x\right\}, \quad \text { for } \quad x \geq 0, \lambda_{i}>0, i=1,2 .
$$

Then $f_{X_{i}}(x)=\lambda_{i} \exp \left\{-\lambda_{i} x\right\}, r_{X_{i}}(x)=\lambda_{i}$ and $f_{X_{i}}(0)=r_{X_{i}}(0)=\lambda_{i} \cdot i=1,2$. From (5.20c) we obtain the first restrictions

$$
\max \left(\lambda_{1}, \lambda_{2}\right) \leq a_{0} \leq \lambda_{1}+\lambda_{2} .
$$

When $x_{1} \geq x_{2}$ and since $a_{1} \geq 0$ inequality (5.20b) transforms into

$$
0 \leq a_{1} \leq\left(\lambda_{1}+a_{1} x_{2}\right)\left[a_{0}-\lambda_{1}+a_{1}\left(x_{1}-x_{2}\right)+a_{2} x_{2}\right],
$$


for all $x_{1} \geq x_{2} \geq 0$. The function $\left(\lambda_{1}+a_{1} x_{2}\right)\left[a_{0}-\lambda_{1}+a_{1}\left(x_{1}-x_{2}\right)+a_{2} x_{2}\right]$ is non-decreasing and its minimum is equal to $\lambda_{1}\left(a_{0}-\lambda_{1}\right)$ when $x_{1}=x_{2}=0$. Thus,

$$
0 \leq a_{1} \leq \lambda_{1}\left(a_{0}-\lambda_{1}\right)
$$

To find the greatest lower bound $\tau$ for which condition (5.20c) in Theorem 5.3.2 is true, is equivalent to verify when $\lambda_{2}\left(a_{0}-\lambda_{2}\right)+\lambda_{2} a_{0} a_{1} x_{2}+\lambda_{2} a_{0} a_{1} x_{2}^{2} \geq 0$. The last inequality is satisfied when $a_{0} \geq \lambda_{2}$. But we got this lower bound for $a_{0}$ already.

Analogously, for $x_{2} \geq x_{1}$ we obtain $0 \leq a_{2} \leq \lambda_{2}\left(a_{0}-\lambda_{2}\right)$.

Summarizing, the parameter constraints are

$$
\max \left(\lambda_{1}, \lambda_{2}\right) \leq a_{0} \leq \lambda_{1}+\lambda_{2}, a_{1}+a_{2}>0 \text { and } 0 \leq a_{i} \leq \lambda_{i}\left(a_{0}-\lambda_{i}\right), i=1,2
$$

The bivariate survival function will be absolutely continuous if $a_{0}=\lambda_{1}+\lambda_{2}$, see (5.15). In such case one obtains $a_{i}=\theta_{i} \lambda_{1} \lambda_{2}$ for $\theta_{i} \in(0,1], i=1,2$. With these specific parameters we get from (5.6) the representation

$$
S_{X_{1}, X_{2}}\left(x_{1}, x_{2}\right)= \begin{cases}\exp \left\{-\left[\lambda_{1} x_{1}+\lambda_{2} x_{2}+\lambda_{1} \lambda_{2} x_{2}\left(\theta_{1} x_{1}+\frac{\theta_{2}-\theta_{1}}{2} x_{2}\right)\right]\right\}, & \text { if } x_{1} \geq x_{2} \geq 0, \\ \exp \left\{-\left[\lambda_{1} x_{1}+\lambda_{2} x_{2}+\lambda_{1} \lambda_{2} x_{1}\left(\theta_{2} x_{2}+\frac{\theta_{1}-\theta_{2}}{2} x_{1}\right)\right]\right\}, & \text { if } x_{2} \geq x_{1} \geq 0\end{cases}
$$

which may be named Generalized Gumbel's bivariate exponential distribution.

Observe, that if put $\theta_{1}=\theta_{2}=\theta$ in the last relation we obtain as a particular case the Gumbel's type I bivariate exponential distribution represented by (5.3).

A bivariate survival function with absolutely continuous and singular components can also be constructed when $a_{0}<\lambda_{1}+\lambda_{2}$. Suppose $\lambda_{1}>\lambda_{2}$ and let $a_{0}=\lambda_{1}$. Notice that with this parameter choice the restrictions in (5.20c) are fulfilled. Hence we obtain $a_{1}=0$ and $a_{2}=\theta \lambda_{2}\left(\lambda_{1}-\lambda_{2}\right)$, where $\theta \in$ $(0,1]$. Substituting these parameter values in (5.6) we get

$$
S_{X_{1}, X_{2}}\left(x_{1}, x_{2}\right)= \begin{cases}\exp \left\{-\left[\lambda_{1} x_{1}+\frac{\theta \lambda_{2}\left(\lambda_{1}-\lambda_{2}\right)}{2} x_{2}^{2}\right]\right\}, & \text { if } x_{1} \geq x_{2} \geq 0, \\ \exp \left\{-\left[\left(\lambda_{1}-\lambda_{2}\right) x_{1}+\lambda_{2} x_{2}+\theta \lambda_{2}\left(\lambda_{1}-\lambda_{2}\right) x_{1}\left(x_{2}-\frac{x_{1}}{2}\right)\right]\right\}, & \text { if } x_{2} \geq x_{1} \geq 0 .\end{cases}
$$

This joint distribution has along the line $x_{1}=x_{2}=x \geq 0$ the singular component

$$
S_{X_{1}, X_{2}}^{s i}(x)=\frac{1-\operatorname{Erf}\left(\frac{\lambda_{1}+\theta \lambda_{2}\left(\lambda_{1}-\lambda_{2}\right) x}{\sqrt{2 \theta \lambda_{2}\left(\lambda_{1}-\lambda_{2}\right)}}\right)}{1-\operatorname{Erf}\left(\frac{\lambda_{1}}{\sqrt{2 \theta \lambda_{2}\left(\lambda_{1}-\lambda_{2}\right)}}\right)}
$$

see Proposition (5.2.15). From expression (5.15), its contribution to the joint distribution is

$$
\mathbb{P}\left(X_{1}=X_{2}\right)=\lambda_{2} \sqrt{\frac{\pi}{2 \theta \lambda_{2}\left(\lambda_{1}-\lambda_{2}\right)}} \exp \left\{\frac{\lambda_{1}^{2}}{2 \theta \lambda_{2}\left(\lambda_{1}-\lambda_{2}\right)}\right\}\left[1-\operatorname{Erf}\left(\frac{\lambda_{1}}{\sqrt{2 \theta \lambda_{2}\left(\lambda_{1}-\lambda_{2}\right)}}\right)\right] .
$$

In the next two examples, firstly we will build a bivariate distribution where marginals have increasing failure rate functions. Then we will present a case where one of the marginal failure rate 
functions is increasing and the other is decreasing.

Example 5.3.6 (Increasing failure rate function marginals). Consider

$$
S_{X_{i}}(x)=\exp \left\{-\lambda_{i} x^{2}-\lambda_{3} x\right\} \quad \text { for } \quad x \geq 0, \lambda_{i}>0, \lambda_{3}>0, i=1,2
$$

Since $f_{X_{i}}(x)=\left(2 \lambda_{i} x+\lambda_{3}\right) \exp \left\{-\lambda_{i} x^{2}-\lambda_{3} x\right\}$, then $r_{X_{i}}(x)=2 \lambda_{i} x+\lambda_{3}, i=1,2$, i.e., the marginals have increasing failure rate functions. First restrictions on the parameter space come from inequalities (5.20a) and (5.20c), i.e. $a_{0} \geq \lambda_{3}$ and $a_{i} \geq 2 \lambda_{i}, i=1,2$.

When $x_{1} \geq x_{2} \geq 0$, from (5.20b) we get a nonnegative function that is increasing in $x_{1}$ and $x_{2}$

$$
2 \lambda_{1}-a_{1}-\left[2 \lambda_{1}\left(x_{1}-x_{2}\right)+\lambda_{3}+a_{1} x_{2}\right]\left[\left(2 \lambda_{1}-a_{1}\right)\left(x_{1}-x_{2}\right)+\lambda_{3}-a_{0}-a_{2} x_{2}\right] \geq 0
$$

with a minimum at the point $(0,0)$. Hence we obtain $a_{1} \leq 2 \lambda_{1}+\lambda_{3}\left(a_{0}-\lambda_{3}\right)$.

Analogously, for $x_{1} \geq x_{2} \geq 0$, we get $2 \lambda_{2} \leq a_{2} \leq 2 \lambda_{2}+\lambda_{3}\left(a_{0}-\lambda_{3}\right)$.

Summarizing, we have the constraints

$$
\lambda_{3} \leq a_{0} \leq 2 \lambda_{3} \text { and } 2 \lambda_{i} \leq a_{i} \leq 2 \lambda_{i}+\lambda_{3}\left(a_{0}-\lambda_{3}\right), i=1,2 .
$$

If $a_{0}=2 \lambda_{3}$ we obtain an absolutely continuous bivariate survival function. In this case $a_{i}=$ $2 \lambda_{i}+\theta_{i} \lambda_{3}^{2}, \theta_{i} \in[0,1], i=1,2$ and letting these values in (5.6) one gets

$S_{X_{1}, X_{2}}\left(x_{1}, x_{2}\right)= \begin{cases}\exp \left\{-\left[\lambda_{1} x_{1}^{2}+\lambda_{3} x_{1}+\lambda_{2} x_{2}^{2}+\lambda_{3} x_{2}+\lambda_{3}^{2} x_{2}\left(\theta_{1} x_{1}+\frac{\theta_{2}-\theta_{1}}{2} x_{2}\right)\right]\right\}, & \text { if } x_{1} \geq x_{2} \geq 0, \\ \exp \left\{-\left[\lambda_{2} x_{2}^{2}+\lambda_{3} x_{2}+\lambda_{1} x_{1}^{2}+\lambda_{3} x_{1}+\lambda_{3}^{2} x_{1}\left(\theta_{2} x_{2}+\frac{\theta_{1}-\theta_{2}}{2} x_{1}\right)\right]\right\}, & \text { if } x_{2} \geq x_{1} \geq 0 .\end{cases}$

Observe that the expression of the joint survival function involves a complete second degree polynomial in the exponent. In addition, notice that $\theta_{1}=\theta_{2}=0$ implies independence between $X_{1}$ and $X_{2}$.

A bivariate survival function having absolutely continuous and singular component can also be captured substituting

$$
a_{0}=\left(1+\theta_{0}\right) \lambda_{3}, \quad \theta_{0} \in[0,1), \quad a_{i}=2 \lambda_{i}+\theta_{0} \theta_{i} \lambda_{3}^{2} \quad \text { and } \quad \theta_{i} \in[0,1], i=1,2
$$

in (5.6). Let $\theta_{0}=0$ in the corresponding expressions to get relation (5.5), i.e the distribution from the class of GMO distributions, see Li and Pellerey (2011).

Example 5.3.7 (One marginal has increasing and other has decreasing failure rate function). Let us fix

$$
S_{X_{1}}(x)=\frac{1}{1+x} \text { and } S_{X_{2}}(x)=\exp \left\{-\lambda_{2} x^{2}-\lambda_{3} x\right\} \quad \text { for } \quad x \geq 0, \lambda_{2}>0, \lambda_{3}>0 .
$$

Since $f_{X_{1}}(x)=\frac{1}{(1+x)^{2}}$ we have $r_{X_{1}}(x)=\frac{1}{1+x}$, and $X_{1}$ has decreasing failure rate. We choose the random variable $X_{2}$ to be the same as in Example 5.3.6, e.g. having an increasing failure rate.

From (5.20a) and (5.20c) we have the first restrictions

$$
a_{0} \geq \max \left(1, \lambda_{3}\right), a_{1} \geq 0 \text { and } a_{2} \geq 2 \lambda_{2} .
$$


When $x_{1}=x_{2}=0$, inequality (5.20b) is equivalent to $0 \leq a_{1} \leq a_{0}-2$. In order to have $a_{1} \geq 0$ we should impose $a_{0} \geq 2$ which implies $\lambda_{3} \geq 1$ and the range for $a_{0}$ is

$$
\max \left(2, \lambda_{3}\right) \leq a_{0} \leq 1+\lambda_{3}
$$

For $\left(x_{1}, x_{2}\right) \neq(0,0)$ and $x_{1} \geq x_{2} \geq 0$, the validity of (5.20b) requires that

$$
\left(1+x_{1}-x_{2}\right)\left[a_{0}-a_{1}+\left(a_{2}-a_{1}\right) x_{2}\right]+a_{1} x_{2}\left[a_{0}+a_{1}\left(x_{1}-x_{2}\right)+a_{2} x_{2}\right]\left(1+x_{1}-x_{2}\right)^{2} \geq a_{0}-a_{1} .
$$

The last inequality is satisfied when $0 \leq a_{1} \leq a_{2}$, say.

In Example 5.3.6 we got $2 \lambda_{2} \leq a_{2} \leq 2 \lambda_{2}+\lambda_{3}\left(a_{0}-\lambda_{3}\right)$ when $x_{2} \geq x_{1} \geq 0$. Thus, the parameter space in this case is

$$
\begin{gathered}
\lambda_{3} \geq 1, \max \left(2, \lambda_{3}\right) \leq a_{0} \leq 1+\lambda_{3}, 0 \leq a_{1} \leq a_{0}-2,0 \leq a_{1} \leq a_{2} \\
\text { and } 2 \lambda_{2} \leq a_{2} \leq 2 \lambda_{2}+\lambda_{3}\left(a_{0}-\lambda_{3}\right) .
\end{gathered}
$$

If $a_{0}=1+\lambda_{3} \geq 2$, we can obtain an absolutely continuous bivariate version, substituting $a_{1}=\alpha_{1}\left(\lambda_{3}-1\right), a_{2}=2 \lambda_{2}+\alpha_{2} \theta, \quad \alpha_{i} \in[0,1], \quad i=1,2$ in (5.6).

To ensure that $a_{2} \geq a_{1}$, the parameters involved have to satisfy the inequality $2 \lambda_{2}+\left(\alpha_{2}-\alpha_{1}\right) \lambda_{3}+$ $\alpha_{1} \geq 0$. Hence we obtain

$S_{X_{1}, X_{2}}\left(x_{1}, x_{2}\right)= \begin{cases}\frac{1}{1+x_{1}-x_{2}} \exp \left\{-\left[\left(\lambda_{2}+\frac{\alpha_{2}}{2} \lambda_{3}-\frac{\alpha_{1}}{2}\left(\lambda_{3}-1\right)\right) x_{2}^{2}+\left(1+\lambda_{3}\right) x_{2}+\alpha_{1}\left(\lambda_{3}-1\right) x_{1} x_{2}\right]\right\}, & \text { if } x_{1} \geq x_{2} \geq 0, \\ \exp \left\{-\left[\left(\frac{\alpha_{1}}{2}\left(\lambda_{3}-1\right)-\frac{\alpha_{2}}{2} \lambda_{3}\right) x_{1}^{2}+x_{1}+\lambda_{2} x_{2}^{2}+\lambda_{3} x_{2}+\alpha_{2} \lambda_{3} x_{1} x_{2}\right]\right\}, & \text { if } x_{2} \geq x_{1} \geq 0 .\end{cases}$

Bivariate survival functions with absolutely continuous and singular components do exist by letting $\lambda_{3}>2$ and $a_{0}=\lambda_{3}+\alpha_{0}, \alpha_{0} \in[0,1)$. Now, the restrictions are

$$
0 \leq a_{1} \leq \lambda_{3}+\alpha_{0}-2, \quad 0 \leq a_{1} \leq a_{2} \quad \text { and } \quad 2 \lambda_{2} \leq a_{2} \leq 2 \lambda_{2}+\lambda_{3}\left(a_{0}-\lambda_{3}\right)
$$

The inequality $a_{2} \geq a_{1}$, is equivalent to $2 \lambda_{2}+\left(\alpha_{0} \alpha_{2}-\alpha_{1}\right) \lambda_{3}+\alpha_{1}\left(2-\alpha_{0}\right) \geq 0$.

Setting

$$
a_{1}=\alpha_{1}\left(\lambda_{3}+\alpha_{0}-2\right), \quad a_{2}=2 \lambda_{2}+\alpha_{0} \alpha_{2} \lambda_{3}, \quad \alpha_{i} \in[0,1], \quad i=1,2
$$

in (5.6) one can find the corresponding expression for $S_{X_{1}, X_{2}}\left(x_{1}, x_{2}\right)$.

We finish this section with additional resources of the class $\mathcal{L}(\mathbf{x} ; \mathbf{a})$ : its closure properties.

\subsubsection{Closure properties of the class $\mathcal{L}(\mathrm{x} ; \mathrm{a})$}

Let $\mathcal{L}(\mathbf{x} ; \mathbf{D})$ denote the class of all survival functions given by (5.6) with a parameter space $\Theta(\mathbf{D})$ defined by (5.19), i.e.

$$
D_{0} \in\left[\max \left\{\tau, \max \left(f_{X_{1}}(0), f_{X_{2}}(0)\right)\right\}, f_{X_{1}}(0)+f_{X_{2}}(0)\right]
$$

$D_{1} \geq 0$ and $D_{2} \geq 0$, with $D_{1}+D_{2}>0$. Note that $\Theta(\mathbf{D})$ may be different for different vectors $\mathbf{D}=$ $\left(D_{0}, D_{1}, D_{2}\right)$ satisfying $(5.2)$. 
We will present in the next statement closure properties of the class $\mathcal{L}(\mathbf{x} ; \mathbf{D})$. These properties along with Theorem 5.2.14 and Proposition 5.2.17 can be used to generate families of distributions belonging to the same class as a second step to the methodology developed and illustrated in Section 5.3.2.

Theorem 5.3.8. Denote by $\mathcal{S}, \mathcal{S}_{1}$ and $\mathcal{S}_{2}$ survival functions belonging to the class $\mathcal{L}(\mathbf{x} ; \mathbf{D})$. The following closure properties are fulfilled:

(LS1) If $\mathcal{S}_{1}, \mathcal{S}_{2} \in \mathcal{L}(\mathbf{x} ; \mathbf{D})$ then their product $\mathcal{S}_{1} \mathcal{S}_{2} \in \mathcal{L}(\mathbf{x} ; \mathbf{D})$;

(LS2) If $\mathcal{S} \in \mathcal{L}(\mathbf{x} ; \mathbf{D})$ then $(\mathcal{S})^{c} \in \mathcal{L}(\mathbf{x} ; \mathbf{D})$ for $c \geq 1$;

(LS3) If $\mathcal{S}_{1}, \mathcal{S}_{2} \in \mathcal{L}(\mathbf{x} ; \mathbf{D})$ then $\left(\mathcal{S}_{1}\right)^{c_{1}}\left(\mathcal{S}_{2}\right)^{c_{2}} \in \mathcal{L}(\mathbf{x} ; \mathbf{D})$ for $c_{1}, c_{2} \geq 1$

(LS4) If $S_{Y_{1}, Y_{2}}\left(x_{1}, x_{2}\right)$ belongs to $\mathcal{L}(\mathbf{x} ; \mathbf{D})$ and $\beta>0$, then $S_{X_{1}, X_{2}}\left(x_{1}, x_{2}\right)=S_{Y_{1}, Y_{2}}\left(\beta x_{1}, \beta x_{2}\right)$ also belongs to $\mathcal{L}(\mathbf{x} ; \mathbf{D})$;

(LS5) If $\mathcal{S}_{1}, \mathcal{S}_{2}$ have the same parameter vector $\mathbf{D}$ and belong to $\mathcal{L}(\mathbf{x} ; \mathbf{D})$, then $\frac{\mathcal{S}_{1}+\mathcal{S}_{2}}{2} \in \mathcal{L}(\mathbf{x} ; \mathbf{D})$;

(LS6) If $S_{Y_{1}, Y_{2}}\left(x_{1}, x_{2}\right)$ belongs to $\mathcal{L}(\mathbf{x} ; \mathbf{D})$ and the exponentially distributed random variable $Y_{3}$ is independent of $\left(Y_{1}, Y_{2}\right)$, define $\left(X_{1}, X_{2}\right)=\left(\min \left(Y_{1}, Y_{3}\right), \min \left(Y_{2}, Y_{3}\right)\right)$. Then $S_{X_{1}, X_{2}}\left(x_{1}, x_{2}\right)$ belongs to $\mathcal{L}(\mathbf{x} ; \mathbf{D})$.

Proof. The properties (LS1)-(LS6) follow after applying relation (5.6) for the corresponding case.

Relations (LS1), (LS3), (LS5) and (LS6) can be easily extended to any finite number of survival functions belonging to $\mathcal{L}(\mathbf{x} ; \mathbf{D})$.

If the vector $\left(Y_{1}, Y_{2}\right)$ is independent of the vector $\left(Z_{1}, Z_{2}\right)$ and their survival functions belong to $\mathcal{L}(\mathbf{x} ; \mathbf{D})$ class, the implication (LS1) means that the survival function of the vector $\left(X_{1}, X_{2}\right)=$ $\left[\min \left(Y_{1}, Z_{1}\right), \min \left(Y_{2}, Z_{2}\right)\right]$ also belongs to $\mathcal{L}(\mathbf{x} ; \mathbf{D})$. This fact has applications in series systems. One may observe the similarity of the stochastic representation of $\left(X_{1}, X_{2}\right)$ with those given by (2.9).

The closure properties listed in Theorem 5.3.8 enable us to construct a wealth of bivariate distributions belonging to $\mathcal{L}(\mathbf{x} ; \mathbf{D})$ by using as a base a known distribution from the $\mathcal{L}(\mathbf{x} ; \mathbf{a})$ class defined by (5.2). We will give two applications below.

Example 5.3.9 ((LS1) based construction). Let us assume that a vector $\left(Y_{1}, Y_{2}\right)$ belonging to $\mathcal{L}(\mathbf{x} ; \mathbf{a})$ is already built starting from its (previously known or pre-specified) marginal failure rates $r_{Y_{1}}(x)$ and $r_{Y_{2}}(x)$. Consider a particular joint survival distribution given by

$$
S_{Z_{1}, Z_{2}}\left(x_{1}, x_{2}\right)=\exp \left\{-\left(b_{1} x_{1}+b_{2} x_{1}^{2}+c_{1} x_{2}+c_{2} x_{2}^{2}\right)\right\}, x_{1}, x_{2} \geq 0
$$

that belongs to $\mathcal{L}(\mathbf{x} ; \mathbf{D})$ where $b_{1}, c_{1}, b_{2}, c_{2} \geq 0$. Then the marginal failure rates of $X_{1}$ and $X_{2}$ are given by $r_{X_{1}}(x)=r_{Y_{1}}(x)+r_{Z_{1}}(x)=r_{Y_{1}}(x)+b_{1}+2 b_{2} x$ and $r_{X_{2}}(x)=r_{Y_{2}}(x)+r_{Z_{2}}(x)=$ $r_{Y_{1}}(x)+c_{1}+2 c_{2} x$.

To finalize building procedure, one may use $r_{X_{1}}\left(x_{1}\right)$ and $r_{X_{2}}\left(x_{2}\right)$ to get a joint distribution $\left(X_{1}, X_{2}\right)$ from the class $\mathcal{L}(\mathbf{x} ; \mathbf{D})$, but with different parameter vector $\mathbf{D}$. 
Example 5.3.10 ((LS6) based construction). Let the survival function of the bivariate random vector $\left(Y_{1}, Y_{2}\right)$ have Gumbel's type I bivariate exponential distribution given by (5.3). Also let $Y_{3}$ be exponentially distributed with parameter $\lambda_{3}>0$ and independent of $\left(Y_{1}, Y_{2}\right)$. Therefore,

$$
\left(X_{1}, X_{2}\right)=\left[\min \left(Y_{1}, Y_{3}\right), \min \left(Y_{2}, Y_{3}\right)\right]
$$

belongs to $\mathcal{L}(\mathbf{x} ; \mathbf{D})$ and its survival function is given by

$$
S_{X_{1}, X_{2}}\left(x_{1}, x_{2}\right)= \begin{cases}\exp \left\{-\left(\lambda_{1}+\lambda_{3}\right) x_{1}-\lambda_{2} x_{2}-\theta \lambda_{1} \lambda_{2} x_{1} x_{2}\right\}, & \text { if } x_{1} \geq x_{2} \geq 0 \\ \exp \left\{-\lambda_{1} x_{1}-\left(\lambda_{2}+\lambda_{3}\right) x_{2}-\theta \lambda_{1} \lambda_{2} x_{1} x_{2}\right\}, & \text { if } x_{2} \geq x_{1} \geq 0\end{cases}
$$

Notice that $S_{Y_{1}, Y_{2}}\left(x_{1}, x_{2}\right)$ is absolutely continuous, but $S_{X_{1}, X_{2}}\left(x_{1}, x_{2}\right)$ displays a singular component along the line $x_{1}=x_{2}=x \geq 0$. This survival function will be reconsidered in Examples 7.2.5 and 7.3.18.

Remark 5.3.11 (Expanding Gumbel's bivariate law). The construction in Example 5.3.10 incorporates a singular component into the resulting distribution, which belongs to a wider class of Extended Marshall Olkin (EMO) bivariate distributions introduced in Chapter 3.

It is worth noting that $S_{X_{1}, X_{2}}\left(x_{1}, x_{2}\right)$ given by (5.23), despite being continuous (but not absolutely continuous), preserves the local constancy of the conditional failure rates $r_{1}\left(x_{1}, x_{2}\right)$ and $r_{2}\left(x_{1}, x_{2}\right)$ in a very similar fashion as Gumbel's bivariate distribution (5.3) does. In fact, the components of the hazard gradient vector in this case are

$$
r_{i}\left(x_{1}, x_{2}\right)= \begin{cases}\lambda_{i}+\lambda_{3}+\theta \lambda_{1} \lambda_{2} x_{3-i}, & \text { if } x_{i}>x_{3-i} \geq 0, \\ \text { does not exist } & \text { if } x_{1}=x_{2}=x \geq 0 \\ \lambda_{i}+\theta \lambda_{1} \lambda_{2} x_{3-i}, & \text { if } 0 \leq x_{i}<x_{3-i}\end{cases}
$$

for $i=1,2$. Therefore, we may consider (5.23) as a Gumbel's extended bivariate exponential distribution with a singularity along the line $x_{1}=x_{2}=x \geq 0$ (see also Remark 6.3.6). If substitute $\lambda_{3}=0$ in (5.23), one will get absolutely continuous Gumbel's version (5.3).

Observe that in Example 5.3.5 we got an absolutely continuous version represented by (5.21) which is another generalization of the Gumbel's bivariate law. See related Remark 6.3.6.

\subsection{Alternative representations and generalizations of the class $\mathcal{L}(\mathrm{x} ; \mathrm{a})$}

We will first discuss two equivalent representations of the class $\mathcal{L}(\mathbf{x} ; \mathbf{a})$ based on mean residual life time and reciprocal of the coordinate subtangent vector. In Section 5.4.2 we suggest several possible extensions: analytical, covering the case of censoring and multivariate one.

\subsubsection{Comparable representations}

We will assume in this subsection that $\mathbb{P}\left(X_{1}=X_{2}\right)=0$, i.e. we will consider absolutely continuous bivariate distributions belonging to the class $\mathcal{L}(\mathbf{x} ; \mathbf{a})$.

In the next remarks we outline two versions of relation (5.2) that defines the class $\mathcal{L}(\mathbf{x} ; \mathbf{a})$. Of course, there exist other possibilities depending on the choice: residual variance vector, vitality 
function vector, etc.

Remark 5.4.1 (Mean residual lifetime vector). Shanbhag and Kotz (1987) provided a definition of bivariate mean residual life vector $\mathbf{M}\left(x_{1}, x_{2}\right)$ with components given by (2.18)

$$
M_{i}\left(x_{1}, x_{2}\right)=\mathbb{E}\left[X_{i}-x_{i} \mid X_{1}>x_{i}, X_{2}>x_{2}\right], \quad i=1,2 .
$$

It is shown by above authors that $M_{1}\left(x_{1}, x_{2}\right)$ and $M_{2}\left(x_{1}, x_{2}\right)$ determine the joint distribution of $\left(X_{1}, X_{2}\right)$ uniquely.

Note that the vector $\mathbf{M}\left(x_{1}, x_{2}\right)$ is determinable as a simple descriptive statistics (being mathematical expectation), while the hazard gradient vector $\mathbf{R}\left(x_{1}, x_{2}\right)$ is defined as an instantaneous failure rate and involves limits. Intuitively, the components of $\mathbf{M}\left(x_{1}, x_{2}\right)$ and $\mathbf{R}\left(x_{1}, x_{2}\right)$ are inversely related to each other. For example, if the joint distribution is given by $S_{X_{1}, X_{2}}\left(x_{1}, x_{2}\right)=\exp \left\{-b_{1} x_{1}-b_{2} x_{2}\right\}$ then both vectors have constant components

$$
\left(r_{1}\left(x_{1}, x_{2}\right), r_{2}\left(x_{1}, x_{2}\right)\right)=\left(b_{1}, b_{2}\right) \quad \text { and } \quad\left(M_{1}\left(x_{1}, x_{2}\right), M_{2}\left(x_{1}, x_{2}\right)\right)=\left(b_{1}^{-1}, b_{2}^{-1}\right),
$$

i.e. being really inverse, with products $r_{i}\left(x_{1}, x_{2}\right) M_{i}\left(x_{1}, x_{2}\right)=1, i=1,2$.

In general, the relation of the components of both vectors is

$$
r_{i}\left(x_{1}, x_{2}\right)=\frac{1}{M_{i}\left(x_{1}, x_{2}\right)}+\frac{\partial}{\partial x_{i}} \ln \left[M_{i}\left(x_{1}, x_{2}\right)\right], i=1,2 .
$$

Then relation (5.2) can be equivalently represented by

$$
\frac{1}{M_{1}\left(x_{1}, x_{2}\right)}+\frac{1}{M_{2}\left(x_{1}, x_{2}\right)}+\frac{\partial}{\partial x_{1}} \ln \left[M_{1}\left(x_{1}, x_{2}\right)\right]+\frac{\partial}{\partial x_{2}} \ln \left[M_{2}\left(x_{1}, x_{2}\right)\right]=a_{0}+a_{1} x_{1}+a_{2} x_{2} .
$$

A counterpart to (5.2) in terms of $\mathbf{M}\left(x_{1}, x_{2}\right)$ could be the class of distributions such that

$$
M_{1}\left(x_{1}, x_{2}\right)+M_{2}\left(x_{1}, x_{2}\right)=C_{1}\left(x_{1}\right)+C_{2}\left(x_{2}\right),
$$

for some nonnegative functions $C_{i}\left(x_{i}\right), i=1,2$. For example, consider the vector

$$
\left(M_{1}\left(x_{1}, x_{2}\right), M_{2}\left(x_{1}, x_{2}\right)\right)=\left(K x_{1}+B_{1}\left(x_{2}\right), K x_{2}+B_{2}\left(x_{1}\right)\right)
$$

for a constant $K \in(-\infty, \infty)$ and positive functions $B_{1}\left(x_{2}\right)$ and $B_{2}\left(x_{1}\right)$.

Sankaran (1992) shows that there exist three distributions satisfying the last relation (bivariate Gumbel represented by (5.3), when $K=0$; bivariate Pareto, if $K>0$ and bivariate finite range, whenever $K<0$ ), see his Theorem 3.1, page 94 (see also the characterization result of Roy (2004) just before Remark 2.4.1). Asadi (1999) characterizes one more distribution with a similar property.

It is worth noting that the components of $\mathbf{R}\left(x_{1}, x_{2}\right)$ corresponding to the components of $\mathbf{M}\left(x_{1}, x_{2}\right)$ given above are

$$
\left(r_{1}\left(x_{1}, x_{2}\right), r_{2}\left(x_{1}, x_{2}\right)\right)=\left[\left(C x_{1}+D_{1}\left(x_{2}\right)\right)^{-1},\left(C x_{2}+D_{2}\left(x_{1}\right)\right)^{-1}\right]
$$

where $C=K(1+K)^{-1}$ and $D_{i}\left(x_{j}\right)=B_{i}\left(x_{j}\right)(1+K)^{-1}, \quad i \neq j, i=1,2$. 
Remark 5.4.2 (Reciprocal of the coordinate subtangent vector). The components of the vector valued reciprocal of the coordinate subtangent have been defined by Roy and Roy (2010) as

$$
T_{i}\left(x_{1}, x_{2}\right)=-\frac{\partial}{\partial x_{i}} \ln \left[f_{X_{1}, X_{2}}\left(x_{1}, x_{2}\right)\right], i=1,2,
$$

where $f_{X_{1}, X_{2}}\left(x_{1}, x_{2}\right)$ is the corresponding bivariate density. The authors show that $T_{i}\left(x_{1}, x_{2}\right)=$ $r_{i}\left(x_{1}, x_{2}\right), i=1,2$, if and only if the underlying distribution is a mixture of exponential distributions with density

$$
f_{X_{1}, X_{2}}\left(x_{1}, x_{2}\right)=\frac{1}{\mathbb{E}\left(X_{1} X_{2}\right)} \int_{0}^{\infty} \int_{0}^{\infty} \exp \left\{-\lambda_{1} x_{1}-\lambda_{2} x_{2}\right\} D\left(d \lambda_{1}, d \lambda_{2}\right), \quad \lambda_{1}, \lambda_{2}>0
$$

where $D(.,$.$) is a probability law located on the set \Lambda=\left\{\lambda_{1} \lambda_{2}=\left[\mathbb{E}\left(X_{1} X_{2}\right)\right]^{-1}\right\}$. Therefore, these (equilibrium) distributions belong to the class $\mathcal{L}(\mathbf{x} ; \mathbf{a})$ whenever $T_{1}\left(x_{1}, x_{2}\right)+T_{2}\left(x_{1}, x_{2}\right)=a_{0}+a_{1} x_{1}+$ $a_{2} x_{2}$ according to (5.2).

It happens that the constancy of the bivariate failure rate $\frac{f_{X_{1}, X_{2}}\left(x_{1}, x_{2}\right)}{S_{X_{1}, X_{2}}\left(x_{1}, x_{2}\right)}$ due to Basu (1971) is characterized also by equalities $T_{i}\left(x_{1}, x_{2}\right)=r_{i}\left(x_{1}, x_{2}\right), i=1,2$. As a complement, Nair et al. (2004) characterize the class defined in (5.24) by constancy of $M\left(x_{1}, x_{2}\right)=\mathbb{E}\left[\left(X_{1}-x_{1}\right)\left(X_{2}-x_{2}\right) \mid X_{1}>\right.$ $\left.x_{i}, X_{2}>x_{2}\right]$, the product moment residual life function.

\subsubsection{Extensions}

We list three possible extensions of the class $\mathcal{L}(\mathbf{x} ; \mathbf{a})$ that may be treated as open problems.

- Analytical extensions. We found a very fruitful idea in the recent paper by Nair and Sankaran (2013). The authors construct a bivariate absolutely continuous and symmetric survival function in the form $S_{X_{1}, X_{2}}\left(x_{1}, x_{2}\right)=\exp \left\{-g\left(x_{1}, x_{2}\right)\right\}$ where the continuous function $g\left(x_{1}, x_{2}\right)$ is defined by functional equation $g\left(x_{1}, x_{2}\right)=H_{Y}^{-1}\left(H_{Y}\left(x_{1}\right)+H_{Y}\left(x_{2}\right)\right)$. Here $H_{Y}(x)$ is the cumulative failure rate function of nonnegative random variable $Y$ which satisfies the relation $\mathbb{P}\left(Y>g\left(x_{1}, x_{2}\right) \mid Y>x_{1}\right)=\mathbb{P}\left(Y>x_{2}\right)$, see Proposition 2.1 in Nair and Sankaran (2013). The usual univariate LMP is a particular case if substitute $g\left(x_{1}, x_{2}\right)=x_{1}+x_{2}$ in the last equation. It is known, since Muliere and Scarsini (1987), that in this case $g\left(x_{1}, x_{2}\right)=x_{1} \oplus x_{2}$, where " $\oplus$ " means associative and reducible binary operation, see Section 2.4.

Our suggestion is as follows: To define a kind of BLMP by condition

$$
\mathbb{P}\left(X_{1}>\gamma_{1}\left(x_{1}, t\right), X_{2}>\gamma_{1}\left(x_{2}, t\right) \mid X_{1}>\gamma_{2}(t), X_{2}>\gamma_{2}(t)\right) \text { is independent of } t,
$$

under corresponding compatibility constraints on continuous functions $\gamma_{1}(x, t)$ and $\gamma_{2}(t)$. It is evident that the BLMP $_{1}$ characterized by $(2.7)$ is the result of letting $\gamma_{1}(x, t)=x+t$ and $\gamma_{2}(t)=t$ in the foregoing relation;

- Censoring case. Our considerations did not cover the censoring data case. This can be done by applying the methodology suggested in Dabrowska (1988) who developed a product integral representation of the bivariate survival functions, see her Proposition 2. This decomposition allows one to evaluate bivariate distributions with any kind of marginal supports: purely continuous, purely discrete, or supports that have discontinuities lying along lines 
orthogonal to the coordinate axes. Moreover, the tool can be used for analysis of partially observed data, i.e. in the frequently met in practice case of censoring. In addition, Dabrowska (1988) introduced a bivariate analogue of the Kaplan-Meier estimate of the survival function of the censored failure times. The results in the cited paper can serve as a key-stone approach in order to extend the class $\mathcal{L}(\mathbf{x} ; \mathbf{a})$ of continuous bivariate distributions to the larger class having arbitrary marginal distributions;

- Multivariate extension. Let us consider the $n$-variate nonnegative continuous random vector $\mathbf{X}=\left(X_{1}, \ldots, X_{n}\right), n \geq 2$. The components of the hazard gradient vector are given by

$$
r_{i}\left(x_{1}, \ldots x_{n}\right)=-\frac{\partial}{\partial x_{i}} \ln \left[S_{X_{1}, \ldots, X_{n}}\left(x_{1}, \ldots x_{n}\right)\right], \quad i=1,2, \ldots n .
$$

The class $\mathcal{L}^{(n)}(\mathbf{x} ; \mathbf{a})$ in $n$-dimensional case can be defined by relation

$$
\sum_{i=1}^{n} r_{i}\left(x_{1}, \ldots x_{n}\right)=a_{0}+a_{1} x_{1}+\ldots+a_{n} x_{n}, \quad a_{j} \geq 0, j=0,1, \ldots, n
$$

One could get representation of the survival function $S_{X_{1}, \ldots, X_{n}}\left(x_{1}, \ldots x_{n}\right)$ corresponding to (5.6) in multivariate case, but the expressions are cumbersome. We provide below the analog of Theorem 5.2.11 when $n=3$.

Theorem 5.4.3. If $S_{X_{1}, X_{2}, X_{3}}\left(x_{1}, x_{2}, x_{3}\right)$ is continuous and nonnegative with continuous first partial derivatives, the relation

$$
r_{1}\left(x_{1}, x_{2}, x_{3}\right)+r_{2}\left(x_{1}, x_{2}, x_{3}\right)+r_{3}\left(x_{1}, x_{2}, x_{3}\right)=a_{0}+a_{1} x_{1}+a_{2} x_{2}+a_{3} x_{3}, \quad a_{i} \geq 0, x_{i} \geq 0,
$$

is fulfilled if and only if the corresponding joint survival function is given by

$$
\begin{cases}S_{X_{1}, X_{2}}\left(x_{1}-x_{3}, x_{2}-x_{3}\right) \exp \left\{-a_{0} x_{3}-\left(a_{1} x_{1}+a_{2} x_{2}\right) x_{3}-\frac{a_{3}-a_{1}-a_{2}}{2} x_{3}^{2}\right\}, & \text { if } x_{1}, x_{2} \geq x_{3} \geq 0 \\ S_{X_{1}, X_{3}}\left(x_{1}-x_{2}, x_{3}-x_{2}\right) \exp \left\{-a_{0} x_{2}-\left(a_{1} x_{1}+a_{3} x_{3}\right) x_{2}-\frac{a_{2}-a_{1}-a_{3}}{2} x_{2}^{2}\right\}, & \text { if } x_{1}, x_{3} \geq x_{2} \geq 0 \\ S_{X_{2}, X_{3}}\left(x_{2}-x_{1}, x_{3}-x_{1}\right) \exp \left\{-a_{0} x_{1}-\left(a_{2} x_{2}+a_{3} x_{3}\right) x_{1}-\frac{a_{1}-a_{2}-a_{3}}{2} x_{1}^{2}\right\}, & \text { if } x_{2}, x_{3} \geq x_{1} \geq 0 .\end{cases}
$$

\subsection{Concluding remarks}

In this chapter we recalled known results and the latest progress associated with celebrated BLMP. We introduced the class $\mathcal{L}(\mathbf{x} ; \mathbf{a})$ and characterized it by the following equivalent relations

$$
\text { Lemma } 5.2 .8 \Leftrightarrow \mathcal{L}(\mathbf{x} ; \mathbf{a}) \Leftrightarrow(\mathbf{5 . 2}) \Leftrightarrow(\mathbf{5 . 6})
$$

It was shown that many bivariate distributions, in addition to those possessing $B L M P_{1}$ and $B L M P_{2}$, belong to $\mathcal{L}(\mathbf{x} ; \mathbf{a})$. A building methodology was developed for various scenarios and illustrated with many examples in Section 5.3. We discussed possible extensions and tried to put out open problems in Section 5.4.2. It is question of time to the corresponding successful solutions and further investigations appear in literature. We do believe that a parallel methodology will be elaborated in bivariate and multivariate discrete settings as well. 
Thus, the class $\mathcal{L}(\mathbf{x} ; \mathbf{a})$ helps to profound the BLMP-notion giving possibility to model the aging phenomena in the complement to the "non-aging" one, which fixes the world on equations (2.6) or (5.4), via $B L M P_{1}$ and $B L M P_{2}$ correspondingly. So, our "world" and base is now equation (5.2). Its graphical interpretation can be seen on Figure 5.2.

The introduced class is promising in modeling dynamic aging dependence, being much more realistic than the virtual "non-aging world". The class $\mathcal{L}(\mathbf{x} ; \mathbf{a})$ includes symmetric and asymmetric continuous distributions with possible singularity, those which are positive or negative quadrant dependent, distributions from the GMO and EMO classes, etc.

This huge variety of bivariate distributions would help to choose the "right" model consistent with the physical nature of the observations. The selection of bivariate distribution to be used depends on considerations involving both the physical scenario at hand and the properties of chosen distribution. Of course, nobody can forbid the influence of the subjective intuition and personal point of view of the investigator who takes the final decision.

We would like to recall the attention on the following. The seminal Gumbel's type I bivariate exponential distribution given by (5.3) has a central place in probability theory, being object of many characterization results. To mention some of them: it is the only absolutely continuous bivariate distribution possessing $B L M P_{2}$, a key bivariate extreme value distribution and it belongs to the class $\mathcal{L}(\mathbf{x} ; \mathbf{a})$. See also Section 2.4 for other characterizations of this distribution. We got two extensions of the Gumbel's type I distribution: one being absolutely continuous and the other not, represented by (5.21) and (5.23) respectively, consult Remark 5.3.11. We do believe that further characterizations based on relations (5.21) and (5.23) will elevate these generalized Gumbel's laws as a new starting point and base for richer bivariate models, with higher flexibility and chances to model the genuine dependence structure.

We did not consider the inference part related with the class $\mathcal{L}(\mathbf{x} ; \mathbf{a})$ given by (5.2), nor its application for real data set. Difficulties would come in the case of continuous distributions with singular part. In general, one may make use of techniques for estimation of multivariate density function with partially differentiable kernels, e.g. Scott (1992). Another option is to apply the Kaplan-Meier estimate of bivariate survival function, even in the case of censoring, following Dabrowska (1988), for example. Possible local linearities or local/global constancies of the observed data can be examined from the corresponding empirical estimates. Once the model is selected, goodness of the fit can be tested with conventional methods. 


\section{Chapter 6}

\section{Linear Sibuya Type Bivariate Lack-of-memory Property}

\subsection{Introduction}

In Chapter 5 we considered two classical versions of bivariate lack-of-memory property:

1. the $B L M P_{1}$, where the joint distributions of $\mathbf{X}_{t}=\left[X_{1}-t, X_{2}-t \mid X_{1}>t, X_{2}>t\right]$ and $\mathbf{X}=\left(X_{1}, X_{2}\right)$ as well as their marginals coincide and do not depend on $t>0$, and

2. the local univariate lack of memory, denoted by $B L M P_{2}$, preserving the univariate LMP for the conditional distributions $\left[X_{i} \mid X_{3-i}>x_{3-i}\right], i=1,2$, as in relation (5.4).

In this chapter we profound the notion of BLMP by introducing a version of lack of memory that joins three objects: a new concept of bivariate lack-of-memory property, continuity (including absolutely continuous distributions or distributions having absolutely continuous and singular parts) and arbitrary nonnegative marginals.

The new notion of the bivariate lack-of-memory property is based on the flexible and (possibly) asymmetric class of bivariate continuous nonnegative distributions with joint survival function specified by the relation

$$
S_{X_{1}, X_{2}}\left(x_{1}+t, x_{2}+t\right)=S_{X_{1}, X_{2}}\left(x_{1}, x_{2}\right) S_{X_{1}, X_{2}}(t, t) B\left(x_{1}, x_{2}, t\right), \quad x_{1}, x_{2} \geq 0, t \geq 0 .
$$

It can be recognized that members of this class may have a singular component (or not) depending on the specific building scheme chosen.

The continuous function $B\left(x_{1}, x_{2}, t\right)$ should satisfy the boundary conditions

$$
B\left(x_{1}, x_{2}, 0\right)=B(0,0, t)=1 .
$$

It may be decreasing or increasing in $x_{1}$ and $x_{2}$ and in $t$ depending of parameters of distributions involved. The function $B\left(x_{1}, x_{2}, t\right)$ is named "aging factor" by Ghurye (1987), see page 457 in Balakrishnan and Lai (2009) as well.

Without some simplifying assumptions on $B\left(x_{1}, x_{2}, t\right)$ such a class is too cumbersome to be of use. We will therefore perform our analysis for an aging function of the form $B\left(x_{1}, x_{2}, t\right)=$ $\exp \left\{-a_{1} x_{1} t-a_{2} x_{2} t\right\}$, where $a_{1}$ and $a_{2}$ are given nonnegative constants. This choice introduces 
a specific aging effect on distribution which will be discussed in Section 6.3. Of course, other expressions for $B\left(x_{1}, x_{2}, t\right)$ are possible, depending on the real problem to be modeled, and are considered in Chapter 7.

To justify this specific choice of the "aging factor", let us consider a system with two elements with lifetimes given by nonnegative random variables $X_{1}$ and $X_{2}$. We view $\left(x_{1}, x_{2}\right)$ as a realization of a continuous random vector $\left(X_{1}, X_{2}\right)$ taking values on the first quadrant $\mathbf{R}_{+}^{2}=$ $\left\{\left(x_{1}, x_{2}\right) \mid x_{1} \geq 0, x_{2} \geq 0\right\}$. Suppose that during the first $t$ units of time the system is protected by breakdowns (by warranty or insurance, say). It is natural to assume that $t<\min \left(x_{1}, x_{2}\right)$. After these first $t$ units of time the system can be affected by two independent "fatal shocks" governed by homogeneous Poisson processes: the $i$-th unit is damaged with intensity $a_{i} t, i=1,2$, see Figure 6.1. The probability of the system survival is given by

$$
S_{X_{1}, X_{2}}\left(x_{1}+t, x_{2}+t\right)=S_{X_{1}, X_{2}}(t, t) S_{X_{1}, X_{2}}\left(x_{1}, x_{2}\right) \exp \left\{-a_{1} x_{1} t-a_{2} x_{2} t\right\}
$$

for all $x_{1}, x_{2} \geq 0$ and $t \geq 0$.

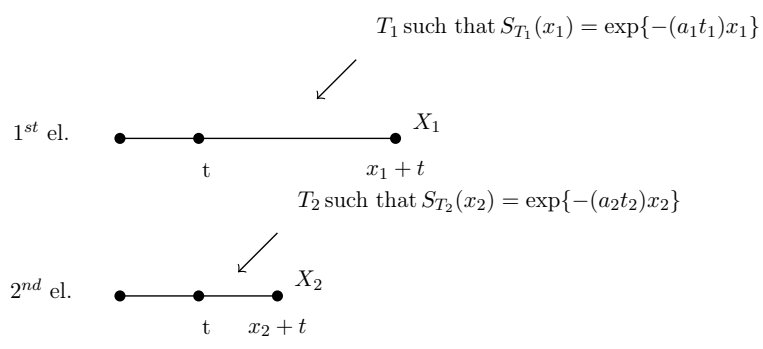

Figure 6.1: An interpretation for relation (6.3).

Remark 6.1.1. As an interpretation, the right hand side in (6.3) is the probability $S_{X_{1}, X_{2}}(t, t)$ that both elements survive the protected initial $t$ units of time multiplied by $S_{X_{1}, X_{2}}\left(x_{1}, x_{2}\right) \exp \left\{-a_{1} x_{1} t-\right.$ $\left.a_{2} x_{2} t\right\}$ - the probability of absence of shocks during the following $x_{i}$ units of time for the $i$-th element, $i=1,2$.

Two potential applications of the scenario described are given below:

- Let a vehicle have a warranty period of $t$ units of time, $t=3$ years, say. During this period, any problem with the car is repaired at no cost to its owner. Later, suppose the proprietary is concerned with problems in the engine as well as in the transmission of the car. Based on the probabilities of occurrence of such problems and associated costs to repair, the decision when to sell the car after warranty period is taken.

- Consider a bank deciding upon lending money in a scheme that the first of several payments is due $t$ months from today. The decision is taken based on the expert analysis of the solvency of the debtor and its collateral after this period.

In Section 6.2 we define a new, Sibuya-type of bivariate lack-of-memory property based on relation (6.3). We characterize that property and show that it is equivalent to the class of distributions $\mathcal{L}(\mathbf{x} ; \mathbf{a})$ introduced in Chapter 5. A related survival copula relation is obtained. In Section 6.3 we use the closure properties presented in Theorem 5.3.8 as a base for constructing related bivariate distributions. Reliability properties treating the aging phenomena are discussed as well. We finish with a short discussion. 


\subsection{A new notion of BLMP}

In this section we will define a new version of the BLMP which also characterizes the class $\mathcal{L}(\mathbf{x} ; \mathbf{a})$ introduced in Chapter 5 . We will obtain a relation between survival copulas of the continuous random vector $\mathbf{X}=\left(X_{1}, X_{2}\right)$, which belongs to $\mathcal{L}(\mathbf{x} ; \mathbf{a})$, and of its corresponding residual lifetime vector $\mathbf{X}_{t}$, as well.

\subsubsection{Linear Sibuya-type BLMP}

Let $S_{X_{1 t}}\left(x_{1}\right)$ and $S_{X_{2 t}}\left(x_{2}\right)$ be the marginal survival functions of residual lifetime vector $\mathbf{X}_{t}$ given by relation (3.13). We have

$$
S_{X_{1 t}}\left(x_{1}\right)=\frac{S_{X_{1}, X_{2}}\left(x_{1}+t, t\right)}{S_{X_{1}, X_{2}}(t, t)} \quad \text { and } \quad S_{X_{2 t}}\left(x_{2}\right)=\frac{S_{X_{1}, X_{2}}\left(t, x_{2}+t\right)}{S_{X_{1}, X_{2}}(t, t)} .
$$

In fact, we will require in Definition 6.2.1 that the baseline vector $\mathbf{X}=\left(X_{1}, X_{2}\right)$ and its residual lifetime vector $\mathbf{X}_{t}$ have the same dependence function $H_{X_{1}, X_{2}}\left(x_{1}, x_{2}\right)$ given by (3.4), which does not depend on $t>0$. Hence, our aim is to transfer the memory-less property of the distribution of the vector $\mathbf{X}_{t}$ or conditional distributions into the "lacking of memory" dependence function $H_{X_{1}, X_{2}}\left(x_{1}, x_{2}\right)$ corresponding to $\left(X_{1}, X_{2}\right)$ via (3.4). Such an approach has not been considered in the literature for the best of our knowledge.

Thus, under the above notations, we propose the following definition.

Definition 6.2.1. The continuous bivariate distribution of $\mathbf{X}=\left(X_{1}, X_{2}\right)$ possesses Linear Sibuyatype BLMP (to be abbreviated LS-BLMP) if and only if

$$
\frac{S_{\mathbf{X}_{t}}\left(x_{1}, x_{2}\right)}{S_{X_{1 t}}\left(x_{1}\right) S_{X_{2 t}}\left(x_{2}\right)}=\frac{S_{X_{1}, X_{2}}\left(x_{1}, x_{2}\right)}{S_{X_{1}}\left(x_{1}\right) S_{X_{2}}\left(x_{2}\right)}
$$

for all $x_{1}, x_{2} \geq 0, t \geq 0$ and

$$
S_{X_{i t}}\left(x_{i}\right)=S_{X_{i}}\left(x_{i}\right) \exp \left\{-a_{i} x_{i} t\right\} \quad \text { for } \quad a_{i}>0, i=1,2 .
$$

Remark 6.2.2. We named the BLMP in Definition 6.2.1 Sibuya-type referring to the work of Sibuya (1960) who considered bivariate distributions written as a product of the two marginals and dependence function $\Psi_{X_{1}, X_{2}}^{F}\left(x_{1}, x_{2}\right)$ defined for $x_{1}, x_{2} \geq 0$ via

$$
\Psi_{X_{1}, X_{2}}^{F}\left(x_{1}, x_{2}\right) F_{X_{1}}\left(x_{1}\right) F_{X_{2}}\left(x_{2}\right)=F_{X_{1}, X_{2}}\left(x_{1}, x_{2}\right) \quad \text { or } \quad \Psi_{X_{1}, X_{2}}^{F}\left(x_{1}, x_{2}\right)=\frac{F_{X_{1}, X_{2}}\left(x_{1}, x_{2}\right)}{F_{X_{1}}\left(x_{1}\right) F_{X_{2}}\left(x_{2}\right)} .
$$

The function $F_{X_{1}, X_{2}}\left(x_{1}, x_{2}\right)$ is the joint distribution of $X_{1}$ and $X_{2}$ with marginal distributions $F_{X_{1}}\left(x_{1}\right)$ and $F_{X_{2}}\left(x_{2}\right)$. A similar expression in terms of survival functions, with dependence function $\Psi_{X_{1}, X_{2}}^{S}\left(x_{1}, x_{2}\right)$, say, is valid too, i.e.

$$
S_{X_{1}, X_{2}}\left(x_{1}, x_{2}\right)=S_{X_{1}}\left(x_{1}\right) S_{X_{2}}\left(x_{2}\right) \Psi_{X_{1}, X_{2}}^{S}\left(x_{1}, x_{2}\right)
$$

In other words, any bivariate survival function may be decomposed as a product of marginal survival functions (representing the independence case) and a dependence function $\Psi_{X_{1}, X_{2}}^{S}\left(x_{1}, x_{2}\right)$ (see 
Proposition 3.2.5 for a related result). Due to relation $\Psi_{X_{1}, X_{2}}^{S}\left(x_{1}, x_{2}\right)=\exp \left\{H_{X_{1}, X_{2}}\left(x_{1}, x_{2}\right)\right\}$, we call the corresponding BLMP "Sibuya-type".

Remark 6.2.3. Equation (6.3) profounds the usual Sibuya-type decomposition given by (6.7) in the following sense: The joint distribution $S_{X_{1}, X_{2}}\left(x_{1}+t, x_{2}+t\right)$ can be represented by a product of bivariate survival functions with non-overlapping arguments and an aging function, $\exp \left\{-a_{1} x_{1} t-\right.$ $\left.a_{2} x_{2} t\right\}$ in our case.

It is direct to verify that condition (6.5) is equivalent to

$$
\frac{S_{X_{1}, X_{2}}\left(x_{1}+t, x_{2}+t\right)}{S_{X_{1}, X_{2}}\left(x_{1}+t, t\right) S_{X_{1}, X_{2}}\left(t, x_{2}+t\right)}=\frac{S_{X_{1}, X_{2}}\left(x_{1}, x_{2}\right)}{S_{X_{1}}\left(x_{1}\right) S_{X_{2}}\left(x_{2}\right) S_{X_{1}, X_{2}}(t, t)} .
$$

Now we will show the importance of representation (6.3) in connection with the LS-BLMP introduced by relations (6.5) and (6.6) in Definition 6.2.1.

Theorem 6.2.4. Relation (6.3) characterizes the LS-BLMP specified by Definition 6.2.1.

Proof. Let (6.3) be true. Put $x_{i}=0$ in (6.3) to get $\exp \left\{-a_{j} x_{j} t\right\}=\frac{S_{X_{j t}}\left(x_{j}\right)}{S_{X_{j}}\left(x_{j}\right)}$ for $i \neq j, i, j=1,2$, which is (6.6), in fact. Substitute the last exponents in (6.3) to obtain relation (6.5). Conversely, let (6.5) and (6.6) be fulfilled. Substitute expression (6.6) in the left hand side of (6.5) to recover the representation $(6.3)$.

The linearity with respect to $x_{1}$ and $x_{2}$ in the exponent participating on (6.3) was our motivation to add the term Linear before Sibuya-BLMP in Definition 6.2.1.

Of course, there exist joint distributions that satisfy (6.5) but not (6.6) and therefore do not possess LS-BLMP according to Definition 6.2.1. A simple illustration is given in the following example.

Example 6.2.5. Let $X_{1}$ and $X_{2}$ be two nonnegative, absolutely continuous and independent random variables that are not exponentially distributed. Their absolutely continuous joint distribution does not satisfy $B L M P_{1}$ nor $B L M P_{2}$. Because of independence between $X_{1}$ and $X_{2}$, the components of the corresponding residual lifetime vector $\mathbf{X}_{t}=\left(X_{1 t}, X_{2 t}\right)$ are also independent. In this case the joint distribution of $\left(X_{1}, X_{2}\right)$ and $\mathbf{X}_{t}$ satisfies (6.5). But since (6.6) does not hold true, the LS-BLMP is not verified.

Remark 6.2.6. Let us analyse the condition (6.6) in Definition 6.2.1 for $i=1$. From (6.4) it may be rewritten as

$$
S_{X_{1 t}}\left(x_{1}\right)=\frac{S_{X_{1}, X_{2}}\left(x_{1}+t, t\right)}{S_{X_{1}, X_{2}}(t, t)}=S_{X_{1}}\left(x_{1}\right) \exp \left\{-a_{1} x_{1} t\right\}
$$

i.e.

$$
S_{X_{1}, X_{2}}\left(x_{1}+t, t\right)=S_{X_{1}}\left(x_{1}\right) S_{X_{1}, X_{2}}(t, t) \exp \left\{-a_{1} x_{1} t\right\} .
$$

First note that $S_{X_{1}, X_{2}}(t, t)$ is the survival function of the random variable $X=\min \left\{X_{1}, X_{2}\right\}$, to be denoted by $S_{X}(t)$. Therefore $S_{X_{1}, X_{2}}\left(x_{1}+t, t\right)$ may be represented as a product of univariate survival functions $S_{X_{1}}\left(x_{1}\right)$ and $S_{X}(t)=S_{X_{1}, X_{2}}(t, t)$ and an "aging" function given by $\exp \left\{-a_{1} x_{1} t\right\}$.

To give another interpretation, observe that $X_{1 t}$ (being the first element of residual lifetime vector $\mathbf{X}_{t}$ ) admits a stochastic representation $X_{1 t}=\min \left\{X_{1}, Z_{1}\right\}$ where $Z_{1}$ is exponentially distributed with parameter $a_{1} t$ and independent of $X_{1}$. Similar conclusion is valid for $S_{X_{2 t}}\left(x_{2}\right)$. 
The following two examples illustrate the importance and generality of the LS-BLMP in comparison to the $\mathrm{BLMP}_{1}$ or $\mathrm{BLMP}_{2}$.

Example 6.2.7. All bivariate distributions possessing the BLMP $P_{1}$ also exhibit LS-BLMP. Gumbel's type I bivariate exponential (5.3) possesses $B L M P_{2}$ and $L S$-BLMP. The product of two bivariate survival functions possessing $B L M P_{1}$ results a new distribution with $B L M P_{1}$. Similarly, the product of two bivariate Gumbel's distributions defined by (5.3) possesses BLMP $P_{2}$. Therefore, the class of distributions possessing $B L M P_{1}$ is closed under the product operation. The same happens with the class of $B L M P_{2}$ distributions. Additionally, in both cases LS-BLMP is preserved.

Example 6.2.8. Multiplying a survival function possessing $B L M P_{1}$ with Gumbel's type I bivariate exponential given by (5.3) one obtains a new survival function that does not exhibit neither BLMP nor $B L M P_{2}$, but satisfies $L S-B L M P$.

In fact, Examples 6.2.7 and 6.2.8 are particular cases of the closure properties of the LS-BLMP distributions discussed in Section 5.3.

Observe that Theorem 5.2.11 states that the class $\mathcal{L}(\mathbf{x} ; \mathbf{a})$ specified by $(5.2)$ is characterized by the joint survival function $S_{X_{1}, X_{2}}\left(x_{1}, x_{2}\right)$ given by (5.6). Second, it was shown in Theorem 6.2.4 that LS-BLMP can be equivalently represented by (6.3). To close the circle and to conclude that LS-BLMP is characterized by the class $\mathcal{L}(\mathbf{x} ; \mathbf{a})$, we will demonstrate in Theorem 6.2.9 that relations (5.6) and (6.3) are equivalent as well.

The next characterization theorem serves as a bridge between relation (5.6) and LS-BLMP. The result helps to give an alternative representation for distributions belonging to the class $\mathcal{L}(\mathbf{x} ; \mathbf{a})$ and serves as a tool for their generation.

Theorem 6.2.9. The joint survival function of $\left(X_{1}, X_{2}\right)$ is given by (5.6) if and only if (6.3) is satisfied.

Proof. Notice that from (5.6) we have $S_{X_{1}, X_{2}}(t, t)=\exp \left\{-a_{0} t-\frac{a_{1}+a_{2}}{2} t^{2}\right\}$. In addition, applying (5.6) for $S_{X_{1}, X_{2}}\left(x_{1}+t, x_{2}+t\right)$ we obtain

$S_{X_{1}, X_{2}}\left(x_{1}+t, x_{2}+t\right)=S_{X_{1}}\left(x_{1}+t-x_{2}-t\right) \exp \left\{-a_{0}\left(x_{2}+t\right)-a_{1}\left(x_{1}+t\right)\left(x_{2}+t\right)-\frac{a_{2}-a_{1}}{2}\left(x_{2}+t\right)^{2}\right\}$,

which is identical to (6.3). The result is the same if $x_{2} \geq x_{1} \geq 0$.

Conversely, suppose (6.3) holds. Define the function $S\left(x_{1}, x_{2}\right)$ such that

$$
S_{X_{1}, X_{2}}\left(x_{1}, x_{2}\right)=S\left(x_{1}, x_{2}\right) \exp \left\{-\frac{a_{1}}{2} x_{1}^{2}-\frac{a_{2}}{2} x_{2}^{2}\right\} .
$$

Notice that $S\left(x_{1}, x_{2}\right)$ does not need to be a bivariate survival function. Substituting (6.9) in (6.3) we obtain the functional equation

$$
S\left(x_{1}+t, x_{2}+t\right)=S\left(x_{1}, x_{2}\right) S(t, t) .
$$

Fix $x_{1}=x_{2}=x \geq 0$ to get $S(x+t, x+t)=S(x, x) S(t, t)$. From Theorem 3.6 in Sahoo and Kannappan (2011), this functional equation has as solution $S(x, x)=\exp \left(-a_{0} x\right)$ for some constant $a_{0}>0$.

Now consider $x_{1} \geq x_{2} \geq 0$ and let $x_{2}=0$ in (6.10) to obtain $S\left(x_{1}+t, t\right)=S\left(x_{1}, 0\right) S(t, t)$. Put 
$y_{1}=x_{1}+t$ and $y_{2}=t$ to get $S\left(y_{1}, y_{2}\right)=S\left(y_{1}-y_{2}, 0\right) \exp \left\{-a_{0} y_{2}\right\}$. From (6.9) we have

$$
S\left(y_{1}-y_{2}, 0\right)=S_{X_{1}, X_{2}}\left(y_{1}-y_{2}, 0\right) \exp \left\{\frac{a_{1}}{2}\left(y_{1}-y_{2}\right)^{2}\right\}=S_{X_{1}}\left(y_{1}-y_{2}\right) \exp \left\{\frac{a_{1}}{2}\left(y_{1}-y_{2}\right)^{2}\right\} .
$$

Therefore

$$
S_{X_{1}, X_{2}}\left(y_{1}, y_{2}\right) \exp \left\{\frac{a_{1}}{2} y_{1}^{2}+\frac{a_{2}}{2} y_{2}^{2}\right\}=S_{X_{1}}\left(y_{1}-y_{2}\right) \exp \left\{\frac{a_{1}}{2}\left(y_{1}-y_{2}\right)^{2}\right\} \exp \left\{-a_{0} y_{2}\right\},
$$

i.e. $S_{X_{1}, X_{2}}\left(y_{1}, y_{2}\right)$ has the form (5.6).

Identical steps and conclusion follow if $0 \leq x_{1} \leq x_{2}$.

With a direct application of Theorem 6.2.9 we have the following

Corollary 6.2.10. If $S_{X_{1}, X_{2}}\left(x_{1}, x_{2}\right)$ is continuous with continuous first partial derivatives, relation (5.2) is fulfilled if and only if (6.3) is fulfilled.

Remark 6.2.11. In the proof of Theorem 6.2.9, notice that if $S\left(x_{1}, x_{2}\right)$ in (6.9) is a survival function, then it exhibits the $B L M P_{1}$, because of (6.10), and satisfies (5.6) with $a_{1}=a_{2}=0$.

To summarize, the LS-BLMP, being equivalent to relation (6.3), characterizes the class $\mathcal{L}(\mathbf{x} ; \mathbf{a})$ as well.

Remark 6.2.12. Ghurye (1987) investigated a similar multiplicative representation of the joint survival function as given by (6.3), see his Section 4. However, the findings in Ghurye (1987) are particular case of our characterization Theorem 6.2.9. For example, Gumbel's bivariate distribution (5.3) satisfies relation (6.9) but the corresponding $S\left(x_{1}, x_{2}\right)$ is not a survival function. Indeed, (5.3) does not meet equation (4.9) in the cited paper.

The proof of Theorem 6.2.9 indicates a procedure for constructing bivariate distributions belonging to the class $\mathcal{L}(\mathbf{x} ; \mathbf{a})$, using as base a member of $B L M P_{1}$-distributions as follows:

1. Choose a bivariate distribution $\left(Y_{1}, Y_{2}\right)$ possessing $B L M P_{1}$;

2. Following (6.9), multiply $S_{Y_{1}, Y_{2}}\left(x_{1}, x_{2}\right)$ by $\exp \left\{-\frac{a_{1}}{2} x_{1}^{2}-\frac{a_{2}}{2} x_{2}^{2}\right\}, a_{1}, a_{2} \geq 0$, to obtain a new joint distribution that satisfies (5.6) for all $x_{1}, x_{2} \geq 0$.

This procedure is demonstrated by the following example.

Example 6.2.13 (Baseline BLMP 1 distribution used to construct LS-BLMP one). Suppose $\left(Y_{1}, Y_{2}\right)$ follow the bivariate exponential distribution considered in Block and Basu (1974), i.e.

$$
S_{Y_{1}, Y_{2}}\left(x_{1}, x_{2}\right)=\frac{\lambda}{\lambda_{1}+\lambda_{2}} \exp \left\{-\lambda_{1} x_{1}-\lambda_{2} x_{2}-\lambda_{12} \max \left(x_{1}, x_{2}\right)\right\}-\frac{\lambda_{12}}{\lambda_{1}+\lambda_{2}} \exp \left\{-\lambda \max \left(x_{1}, x_{2}\right)\right\},
$$

where the parameters $\lambda_{1}, \lambda_{2}$ and $\lambda_{12}$ are positive and $\lambda=\lambda_{1}+\lambda_{2}+\lambda_{12}$. This distribution is absolutely continuous and possesses BLMP $P_{1}$. Applying (6.9), multiply $S_{Y_{1}, Y_{2}}\left(x_{1}, x_{2}\right)$ by $\exp \left\{-\frac{a_{1}}{2} x_{1}^{2}-\frac{a_{2}}{2} x_{2}^{2}\right\}$, $a_{1}, a_{2} \geq 0$, to obtain

$$
S_{X_{1}, X_{2}}\left(x_{1}, x_{2}\right)= \begin{cases}\frac{\lambda}{\lambda_{1}+\lambda_{2}} \exp \left\{-\lambda_{1} x_{1}-\lambda_{2} x_{2}-\lambda_{12} x_{1}-\frac{a_{1}}{2} x_{1}^{2}-\frac{a_{2}}{2} x_{2}^{2}\right\} & \\ -\frac{\lambda_{12}}{\lambda_{1}+\lambda_{2}} \exp \left\{-\lambda x_{1}-\frac{a_{1}}{2} x_{1}^{2}-\frac{a_{2}}{2} x_{2}^{2}\right\}, & \text { if } x_{1} \geq x_{2} \geq 0, \\ \frac{\lambda}{\lambda_{1}+\lambda_{2}} \exp \left\{-\lambda_{1} x_{1}-\lambda_{2} x_{2}-\lambda_{12} x_{2}-\frac{a_{1}}{2} x_{1}^{2}-\frac{a_{2}}{2} x_{2}^{2}\right\} & \\ -\frac{\lambda_{12}}{\lambda_{1}+\lambda_{2}} \exp \left\{-\lambda x_{2}-\frac{a_{1}}{2} x_{1}^{2}-\frac{a_{2}}{2} x_{2}^{2}\right\}, & \text { if } x_{2} \geq x_{1} \geq 0,\end{cases}
$$


which possesses LS-BLMP according to Theorem 6.2.9. One may check that $S_{X_{1}, X_{2}}\left(x_{1}, x_{2}\right)$ admits the representation given by (5.6): consider $x_{1} \geq x_{2} \geq 0$ and notice that $X_{1}$ has marginal survival function written as

$$
\left.S_{X_{1}}\left(x_{1}\right)=\frac{\lambda}{\lambda_{1}+\lambda_{2}} \exp \left\{-\lambda_{1} x_{1}-\lambda_{12} x_{1}-\frac{a_{1}}{2} x_{1}^{2}\right\}-\frac{\lambda_{12}}{\lambda_{1}+\lambda_{2}} \exp \left\{-\lambda x_{1}-\frac{a_{1}}{2} x_{1}^{2}\right)\right\} .
$$

Using $x_{1}-x_{2}$ instead of $x_{1}$ in the last equation we obtain just the form (5.6) of $S_{X_{1}, X_{2}}\left(x_{1}, x_{2}\right)$ when $\lambda=a_{0}$. By analogy, we obtain the second expression in (5.6) when $x_{2} \geq x_{1} \geq 0$.

\subsubsection{Survival copula representations of the class $\mathcal{L}(\mathrm{x} ; \mathrm{a})$}

Recall that a bivariate copula, denoted by the function $C(u, v):[0,1]^{2} \rightarrow[0,1]$, is a bivariate probability distribution with marginals uniformly distributed on $[0,1]$, and the corresponding survival copula $\bar{C}(u, v)$ is given by

$$
\bar{C}(u, v)=u+v-1+C(1-u, 1-v),
$$

see Definition 2.3.4 and relation (2.14), respectively. The bivariate survival function, its marginals and the corresponding survival copula are related by the equation

$$
S_{X_{1}, X_{2}}\left(x_{1}, x_{2}\right)=\bar{C}\left(S_{X_{1}}\left(x_{1}\right), S_{X_{2}}\left(x_{2}\right)\right)
$$

Since copulas are widely used to describe the dependence between random variables, it is worthwhile to consider how this concept is related with Definition 6.2.1, where it is required that $\mathbf{X}=\left(X_{1}, X_{2}\right)$ and $\mathbf{X}_{t}$ have the same dependence function $H_{X_{1}, X_{2}}\left(x_{1}, x_{2}\right)$, see relation (6.5). Let us first consider the following Lemma.

Lemma 6.2.14. Let $\left(X_{1}, X_{2}\right)$ be a nonnegative bivariate random vector with continuous joint survival function $S_{X_{1}, X_{2}}\left(x_{1}, x_{2}\right)$ and residual lifetime vector $\mathbf{X}_{t}$ given by (3.13). If $S_{X_{1}, X_{2}}\left(x_{1}, x_{2}\right)$ possesses the BLPM $M_{1}$, given by the functional equation (2.6), then $\mathbf{X}=\left(X_{1}, X_{2}\right)$ and $\mathbf{X}_{t}$ have the same survival copula.

Proof. Denote by $\bar{C}(u, v)$ and $\bar{C}_{t}(u, v)$ the survival copulas corresponding to $S_{X_{1}, X_{2}}\left(x_{1}, x_{2}\right)$ and $S_{X_{1 t}, X_{2 t}}\left(x_{1}, x_{2}\right)$, respectively, and suppose the BLMP 1 holds true. Thus, from the definition of residual lifetime vector (3.13) and the functional equation (2.6) we have

$$
S_{X_{1 t}, X_{2 t}}\left(x_{1}, x_{2}\right)=\frac{S_{X_{1}, X_{2}}\left(x_{1}+t, x_{2}+t\right)}{S_{X_{1}, X_{2}}(t, t)}=S_{X_{1}, X_{2}}\left(x_{1}, x_{2}\right)
$$

for all $x_{1}, x_{2} \geq 0$ and $t \geq 0$. Thus, $X_{i}$ and $X_{i t}, i=1,2$, have the same continuous survival functions. From Sklar's Theorem, see Theorem 2.2.3 in Nelsen (2006), we have $\bar{C}(u, v)=\bar{C}_{t}(u, v)$, for all $u, v \in[0,1]$ and $t \geq 0$.

The converse in Lemma 6.2.14 is not true, as shown in the next example.

Example 6.2.15 ( $\mathbf{X}$ and $\mathbf{X}_{t}$ have the same survival copula but BLMP 1 does not hold true). Let us consider the Clayton copula given by $C(u, v ; \delta)=\left(u^{-\delta}+v^{-\delta}-1\right)^{-\frac{1}{\delta}}$, where $u, v \in[0,1]$ and $0<\delta<\infty$, see family $B 4$ in Joe (1997), and two unit exponential marginals, $S_{X_{i}}\left(x_{i}\right)=\exp \left\{-x_{i}\right\}$, 
$i=1,2$, to obtain the joint survival function $S_{X_{1}, X_{2}}\left(x_{1}, x_{2}\right)=\frac{1}{\exp \left\{x_{1}\right\}+\exp \left\{x_{2}\right\}-1}$. The joint survival function of the residual lifetime vector is given by

$$
S_{X_{1 t}, X_{2 t}}\left(x_{1}, x_{2}\right)=\frac{S_{X_{1}, X_{2}}\left(x_{1}+t, x_{2}+t\right)}{S_{X_{1}, X_{2}}(t, t)}=\frac{2-\exp \{-t\}}{\exp \left\{x_{1}\right\}+\exp \left\{x_{2}\right\}-\exp \{-t\}},
$$

and the marginals are $S_{X_{i t}}\left(x_{i}\right)=\frac{2-\exp \{-t\}}{\exp \left\{x_{i}\right\}+1-\exp \{-t\}}, i=1,2$. Letting $u=S_{X_{1 t}}\left(x_{1}\right), v=S_{X_{2 t}}\left(x_{2}\right)$ and noticing that

$$
S_{X_{1 t}, X_{2 t}}\left(x_{1}, x_{2}\right)=\frac{1}{\frac{\exp \left\{x_{1}\right\}+1-\exp \{-t\}}{2-\exp \{-t\}}+\frac{\exp \left\{x_{2}\right\}+1-\exp \{-t\}}{2-\exp \{-t\}}-\frac{2-\exp \{-t\}}{2-\exp \{-t\}}},
$$

using the relation between survival functions and survival copulas (6.11) we obtain $\bar{C}_{t}(u, v)=$ $\bar{C}(u, v)$, for all $u, v \in[0,1]$ and $t \geq 0$. Meanwhile, $S_{X_{1 t}, X_{2 t}}\left(x_{1}, x_{2}\right) \neq S_{X_{1}, X_{1}}\left(x_{1}, x_{2}\right)$ for $t>0$, i.e., $S_{X_{1}, X_{1}}\left(x_{1}, x_{2}\right)$ does not possess $B L M P_{1}$.

In the next theorem we present the relation between the survival copulas of $\mathbf{X}$ and $\mathbf{X}_{t}$ when the bivariate random vector $\mathbf{X}=\left(X_{1}, X_{2}\right)$ belongs to $\mathcal{L}(\mathbf{x} ; \mathbf{a})$.

Theorem 6.2.16. Consider a bivariate random vector $\mathbf{X}=\left(X_{1}, X_{2}\right)$ that belongs to the class $\mathcal{L}(\mathbf{x} ; \mathbf{a})$ and the corresponding residual lifetime vector $\mathbf{X}_{t}$ with survival function given by

$$
S_{\mathbf{X}_{t}}\left(x_{1}, x_{2}\right)=\frac{S_{X_{1}, X_{2}}\left(x_{1}+t, x_{2}+t\right)}{S_{X_{1}, X_{2}}(t, t)}=S_{X_{1}, X_{2}}\left(x_{1}, x_{2}\right) \exp \left\{-a_{1} x_{1} t-a_{2} x_{2} t\right\} .
$$

Denote by $\bar{C}(u, v)$ and $\bar{C}_{t}(u, v)$ the survival copulas of $\mathbf{X}$ and $\mathbf{X}_{t}$, respectively. Then

$$
\begin{aligned}
\bar{C}_{t}(u, v)= & \bar{C}\left(\exp \left\{-H_{X_{1}}\left(G_{1 t}^{-1}(-\ln u)\right)\right\}, \exp \left\{-H_{X_{2}}\left(G_{2 t}^{-1}(-\ln v)\right)\right\}\right) \\
& \times \exp \left\{-a_{1} t G_{1 t}^{-1}(-\ln u)-a_{2} t G_{2 t}^{-1}(-\ln v)\right\},
\end{aligned}
$$

where $G_{i t}(x)=H_{X_{i}}\left(x_{i}\right)+a_{i} x_{i}$ t are the cumulative failure rates of $X_{i t}, i=1,2$.

Proof. From (6.6) the marginals of $\mathbf{X}_{t}$ have survival functions given by $S_{X_{i}}\left(x_{i}\right) \exp \left\{-a_{i} x_{i} t\right\}, i=1,2$. Using the relation between survival functions and survival copulas (6.11), equation (6.13) can be rewritten as

$$
\begin{aligned}
\bar{C}_{t}\left(S_{X_{1}}\left(x_{1}\right) \exp \left\{-a_{1} x_{1} t\right\}, S_{X_{2}}\left(x_{2}\right) \exp \left\{-a_{2} x_{2} t\right\}\right)= & \bar{C}\left(S_{X_{1}}\left(x_{1}\right), S_{X_{2}}\left(x_{2}\right)\right) \\
& \times \exp \left\{-a_{1} x_{1} t-a_{2} x_{2} t\right\} .
\end{aligned}
$$

Let $u=S_{X_{1}}\left(x_{1}\right) \exp \left\{-a_{1} x_{1} t\right\}$ and $v=S_{X_{2}}\left(x_{2}\right) \exp \left\{-a_{2} x_{2} t\right\}$. Since $S_{X_{i}}\left(x_{i}\right)=\exp \left\{-H_{X_{i}}\left(x_{i}\right)\right\}$, $i=1,2$, then $G_{i t}(x)=H_{X_{i}}\left(x_{i}\right)+a_{i} x_{i} t$, so we get $x_{1}=G_{1 t}^{-1}(-\ln u)$ and $x_{2}=G_{2 t}^{-1}(-\ln v)$. Substituting $u$ and $v$ in (6.15) we have relation (6.14).

Corollary 6.2.17. Consider a bivariate random vector $\mathbf{X}=\left(X_{1}, X_{2}\right)$ that belongs to the class $\mathcal{L}(\mathbf{x} ; \mathbf{a})$ and the corresponding residual lifetime vector $\mathbf{X}_{t}$, with survival copulas $\bar{C}(u, v)$ and $\bar{C}_{t}(u, v)$, respectively. If $a_{1}=a_{2}=0$ in (5.2) then $\bar{C}(u, v)=\bar{C}_{t}(u, v)$ for all $u, v \in(0,1]$ and $t \geq 0$.

Proof. Besides relation (5.2), equation (6.3) also characterize the members of $\mathcal{L}(\mathbf{x} ; \mathbf{a})$, see Theorems 6.2.4 and 6.2.9. Thus, considering $a_{1}=a_{2}=0$ in (6.3) we have $S_{X_{1}, X_{2}}\left(x_{1}, x_{2}\right)$ possessing BLMP 1 since the functional equation (2.6) holds true. The result follows from Lemma 6.2.14. 
The next example illustrates an application of Theorem 6.2.16.

Example 6.2.18. Suppose $S_{X_{1}, X_{2}}\left(x_{1}, x_{2}\right)$ belongs to $\mathcal{L}(\mathbf{x} ; \mathbf{a})$ with marginals exponentially distributed, $S_{X_{i}}(x)=\exp \left\{-\lambda_{i} x_{i}\right\}, \lambda_{i}>0, i=1,2$. Then $G_{i t}(x)=\lambda_{i} x_{i}+a_{i} x_{i} t$, and $G_{i t}^{-1}(u)=\frac{u}{\lambda_{i}+a_{i} t}$, $i=1,2$. From (6.14), we have

$$
\bar{C}_{t}(u, v)=\bar{C}\left(\exp \left\{\frac{\lambda_{1} \ln u}{\lambda_{1}+a_{1} t}\right\}, \exp \left\{\frac{\lambda_{2} \ln v}{\lambda_{2}+a_{2} t}\right\}\right) \exp \left\{\frac{a_{1} t \ln u}{\lambda_{1}+a_{1} t}+\frac{a_{2} t \ln v}{\lambda_{2}+a_{2} t}\right\},
$$

which can be simplified to

$$
\bar{C}_{t}(u, v)=\bar{C}\left(u^{\frac{\lambda_{1}}{\lambda_{1}+a_{1} t}}, v^{\frac{\lambda_{2}}{\lambda_{2}+a_{2} t}}\right) u^{\frac{a_{1} t}{\lambda_{1}+a_{1} t}} v^{\frac{a_{2} t}{\lambda_{2}+a_{2} t}}
$$

First let $\left(X_{1}, X_{2}\right)$ follow Gumbel's type I exponential distribution with survival function given by (5.3), so that $S_{X_{1}, X_{2}}\left(x_{1}, x_{2}\right)$ belongs to $\mathcal{L}(\mathbf{x} ; \mathbf{a})$. Therefore, $\bar{C}(u, v)=u v \exp \{-\theta \ln u \ln v\}, \theta \in(0,1]$, i.e., we obtain the Gumbel-Barnett survival copula with parameter $\theta$, see (3.17). Substituting $\bar{C}(u, v)$ in $(6.16)$

$$
\bar{C}_{t}(u, v)=u v \exp \left\{-\frac{\theta}{\left(1+\theta \lambda_{2} t\right)\left(1+\theta \lambda_{1} t\right)} \ln u \ln v\right\} .
$$

Therefore, $\bar{C}_{t}(u, v)$ also follows the Gumbel-Barnett survival copula, but with parameter that depends on $t$. When $t=0$ we recover the survival copula $\bar{C}(u, v)$ of $\left(X_{1}, X_{2}\right)$ and letting $t \rightarrow \infty$, we obtain the independence copula $\bar{C}_{\infty}(u, v)=u v$.

Second, let $S_{X_{1}, X_{2}}\left(x_{1}, x_{2}\right)$ possess $B L M P_{1}$, i.e., $a_{1}=a_{2}=0$ in (6.3), so that $S_{X_{1}, X_{2}}\left(x_{1}, x_{2}\right)=$ $\exp \left\{-\lambda_{1} x_{1}-\lambda_{2} x_{2}\right\}$, corresponding to $\theta=0$ in (5.3). Thus, from (6.16) we obtain $\bar{C}_{t}(u, v)=\bar{C}(u, v)$ for all $t \geq 0$.

In the sequel we obtain a characterizing functional equation involving the survival copula $\bar{C}_{t}$ of $\mathbf{X}_{t}$ for the absolutely continuous members of the class $\mathcal{L}(\mathbf{x} ; \mathbf{a})$.

Theorem 6.2.19. The absolutely continuous random vector $\left(X_{1}, X_{2}\right)$ belongs to the class $\mathcal{L}(\mathbf{x} ; \mathbf{a})$, if and only if there exist nonnegative constants $a_{1}$ and $a_{2}$, such that the functional equation

$$
\bar{C}_{t}\left(\frac{S_{X_{1}, X_{2}}\left(x_{1}+t, t\right)}{S_{X_{1}, X_{2}}(t, t)}, \frac{S_{X_{1}, X_{2}}\left(t, x_{2}+t\right)}{S_{X_{1}, X_{2}}(t, t)}\right)=\bar{C}_{t}\left(S_{X_{1}}\left(x_{1}\right) \exp \left\{-a_{1} x_{1} t\right\}, S_{X_{2}}\left(x_{2}\right) \exp \left\{-a_{2} x_{2} t\right\}\right),
$$

is satisfied for all $x_{1}, x_{2}, t \geq 0$, where the survival copula $\bar{C}_{t}$ of $\mathbf{X}_{t}$ is differentiable in its arguments.

Proof. Let us assume that the functional equation (6.17) is satisfied. We will show that (5.2) is fulfilled. Using the chain rule, we take the derivative of (6.17) with respect to $t$ to obtain

$$
\begin{aligned}
& \bar{C}_{t}^{1}\left(\frac{S_{X_{1}, X_{2}}\left(x_{1}+t, t\right)}{S_{X_{1}, X_{2}}(t, t)}, \frac{S_{X_{1}, X_{2}}\left(t, x_{2}+t\right)}{S_{X_{1}, X_{2}}(t, t)}\right) \frac{\left[S_{X_{1}, X_{2}}^{1}\left(x_{1}+t, t\right)+S_{X_{1}, X_{2}}^{2}\left(x_{1}+t, t\right)\right] S_{X_{1}, X_{2}}(t, t)-S_{X_{1}, X_{2}}\left(x_{1}+t, t\right)\left[S_{X_{1}, X_{2}}^{1}(t, t)+S_{X_{1}}^{2}, X_{2}(t, t)\right]}{\left[S_{X_{1}, X_{2}}(t, t)\right]^{2}}
\end{aligned}
$$

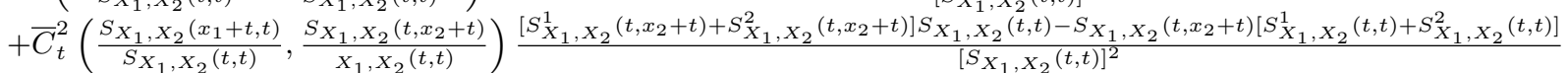

$$
\begin{aligned}
& =\bar{C}_{t}^{1}\left(S_{X_{1}}\left(x_{1}\right) \exp \left\{-a_{1} x_{1} t\right\}, S_{X_{2}}\left(x_{2}\right) \exp \left\{-a_{2} x_{2} t\right\}\right)\left(-a_{1} x_{1} S_{X_{1}}\left(x_{1}\right) \exp \left\{-a_{1} x_{1} t\right\}\right) \\
& +\bar{C}_{t}^{2}\left(S_{X_{1}}\left(x_{1}\right) \exp \left\{-a_{1} x_{1} t\right\}, S_{X_{2}}\left(x_{2}\right) \exp \left\{-a_{2} x_{2} t\right\}\right)\left(-a_{2} x_{2} S_{X_{2}}\left(x_{2}\right) \exp \left\{-a_{2} x_{2} t\right\}\right),
\end{aligned}
$$

where the superscripts ${ }^{1}$ and ${ }^{2}$ denote the partial derivatives with respect to the first and second 
arguments of the corresponding functions. Letting $x_{1}=0$ in last equation we have

$$
\begin{aligned}
& \bar{C}_{t}^{2}\left(1, \frac{S_{X_{1}, X_{2}}\left(t, x_{2}+t\right)}{S_{X_{1}, X_{2}}(t, t)}\right) \frac{\left[S_{X_{1}, X_{2}}^{1}\left(t, x_{2}+t\right)+S_{X_{1}, X_{2}}^{2}\left(t, x_{2}+t\right)\right] S_{X_{1}, X_{2}}(t, t)-S_{X_{1}, X_{2}}\left(t, x_{2}+t\right)\left[S_{X_{1}, X_{2}}^{1}(t, t)+S_{X_{1}, X_{2}}^{2}(t, t)\right]}{\left[S_{X_{1}, X_{2}}(t, t)\right]^{2}} \\
& =\bar{C}_{t}^{2}\left(1, S_{X_{2}}\left(x_{2}\right) \exp \left\{-a_{2} x_{2} t\right\}\right)\left(-a_{2} x_{2} S_{X_{2}}\left(x_{2}\right) \exp \left\{-a_{2} x_{2} t\right\}\right) .
\end{aligned}
$$

When $x_{i}=0$ in (6.17) we get relations (6.6) in Definition 6.2.1, $i=1,2$ and therefore

$$
\begin{aligned}
& \frac{\left[S_{X_{1}, X_{2}}^{1}\left(t, x_{2}+t\right)+S_{X_{1}, X_{2}}^{2}\left(t, x_{2}+t\right)\right] S_{X_{1}, X_{2}}(t, t)-S_{X_{1}, X_{2}}\left(t, x_{2}+t\right)\left[S_{X_{1}, X_{2}}^{1}(t, t)+S_{X_{1}, X_{2}}^{2}(t, t)\right]}{\left[S_{X_{1}, X_{2}}(t, t)\right]^{2}} \\
& =-a_{2} x_{2} S_{X_{2}}\left(x_{2}\right) \exp \left\{-a_{2} x_{2} t\right\} .
\end{aligned}
$$

Since $r\left(t, x_{2}+t\right)=-\frac{S_{X_{1}, X_{2}}^{1}\left(t, x_{2}+t\right)+S_{X_{1}, X_{2}}^{2}\left(t, x_{2}+t\right)}{S_{X_{1}, X_{2}}\left(t, x_{2}+t\right)}$ and $r(t, t)=-\frac{S^{1}(t, t)+S^{2}(t, t)}{S(t, t)}$ we get

$$
-\frac{S_{X_{1}, X_{2}}\left(t, x_{2}+t\right)}{S_{X_{1}, X_{2}}(t, t)}\left[r\left(t, x_{2}+t\right)-r(t, t)\right]=-a_{2} x_{2} S_{X_{2}}\left(x_{2}\right) \exp \left\{-a_{2} x_{2} t\right\},
$$

which is equivalent to

$$
r\left(t, x_{2}+t\right)=r(t, t)+a_{2} x_{2} .
$$

Analogously we obtain the equation

$$
r\left(x_{1}+t, t\right)=r(t, t)+a_{1} x_{1} .
$$

Now we will represent $r(t, t)$ as a function of $a_{0}, a_{1}, a_{2}$ and $t$. Taking the partial derivative of (6.17) with respect to $x_{1}$ we have

$$
\begin{aligned}
\bar{C}_{t}^{1}\left(\frac{S_{X_{1}, X_{2}}\left(x_{1}+t, t\right)}{S_{X_{1}, X_{2}}(t, t)}, \frac{S_{X_{1}, X_{2}}\left(t, x_{2}+t\right)}{S_{X_{1}, X_{2}}(t, t)}\right) \frac{S_{X_{1}, X_{2}}^{1}\left(x_{1}+t, t\right)}{S_{X_{1}, X_{2}}(t, t)} & =\bar{C}_{t}^{1}\left(S_{X_{1}}\left(x_{1}\right) \exp \left\{-a_{1} x_{1} t\right\}, S_{X_{2}}\left(x_{2}\right) \exp \left\{-a_{2} x_{2} t\right\}\right) \\
& \times\left(-f_{X_{1}}\left(x_{1}\right) \exp \left\{-a_{1} x_{1} t\right\}-a_{1} t S_{X_{1}}\left(x_{1}\right) \exp \left\{-a_{1} x_{1} t\right\}\right),
\end{aligned}
$$

where $f_{X_{1}}\left(x_{1}\right)$ is the density of $X_{1}$. Applying (6.6) in last equation we obtain

$$
\frac{S_{X_{1}, X_{2}}^{1}\left(x_{1}+t, t\right)}{S_{X_{1}, X_{2}}(t, t)}=-f_{X_{1}}\left(x_{1}\right) \exp \left\{-a_{1} x_{1} t\right\}-a_{1} t S_{X_{1}}\left(x_{1}\right) \exp \left\{-a_{1} x_{1} t\right\}
$$

and fixing $x_{1}=0$ we arrive to

$$
r_{1}(t, t)=f_{X_{1}}(0)+a_{1} t
$$

Similarly we have

$$
r_{2}(t, t)=f_{X_{2}}(0)+a_{2} t
$$

where $f_{X_{2}}\left(x_{2}\right)$ is the density of $X_{2}$. The sum of last two equations gives

$$
r(t, t)=r_{1}(t, t)+r_{2}(t, t)=\left[f_{X_{1}}(0)+f_{X_{2}}(0)\right]+a_{1} t+a_{2} t .
$$

Let $t=0$ in last relation to get $f_{X_{1}}(0)+f_{X_{2}}(0)=a_{0} \geq 0$. Thus,

$$
r(t, t)=a_{0}+a_{1} t+a_{2} t .
$$

Taking into account (6.18) and (6.19) we conclude that $r\left(x_{1}, x_{2}\right)=a_{0}+a_{1} x_{1}+a_{2} x_{2}$. Therefore, we obtain relation (5.2) which defines the class $\mathcal{L}(\mathbf{x} ; \mathbf{a})$. In addition, corresponding bivariate distribu- 
tions are absolutely continuous because of equation $f_{X_{1}}(0)+f_{X_{2}}(0)=a_{0}$, see Remark 5.2.18.

Conversely, assume that the random vector $\left(X_{1}, X_{2}\right)$ belonging to the class $\mathcal{L}(\mathbf{x} ; \mathbf{a})$ is absolutely continuous. From Theorem 6.2.4 we have (6.3) equivalent to (6.5) and (6.6). Applying Sklar's theorem to (6.3) we obtain the functional equation (6.17).

In the next example we find a particular form of functional equation (6.17).

Example 6.2.20. Let us consider the absolutely continuous joint survival function given by (5.21),

$$
S_{X_{1}, X_{2}}\left(x_{1}, x_{2}\right)= \begin{cases}\exp \left\{-\left[\lambda_{1} x_{1}+\lambda_{2} x_{2}+\lambda_{1} \lambda_{2} x_{2}\left(\theta_{1} x_{1}+\frac{\theta_{2}-\theta_{1}}{2} x_{2}\right)\right]\right\}, & \text { if } x_{1} \geq x_{2} \geq 0 \\ \exp \left\{-\left[\lambda_{1} x_{1}+\lambda_{2} x_{2}+\lambda_{1} \lambda_{2} x_{1}\left(\theta_{2} x_{2}+\frac{\theta_{1}-\theta_{2}}{2} x_{1}\right)\right]\right\}, & \text { if } x_{2} \geq x_{1} \geq 0\end{cases}
$$

where $\theta_{i} \in(0,1]$, and $\lambda_{i}>0, i=1,2$.

The marginal survival functions are $S_{X_{i}}\left(x_{i}\right)=\exp \left\{-\lambda_{i} x_{i}\right\}, i=1,2$. The survival function of the residual lifetime vector $\mathbf{X}_{t}$ can be obtained from (6.3). After some algebra we get the corresponding survival copula

$$
\bar{C}_{t}(u, v)= \begin{cases}u v \exp \left\{-\frac{\theta_{1}}{\gamma_{1}(t) \gamma_{2}(t)} \ln u \ln v\right\} \exp \left\{-\frac{\lambda_{1}\left(\theta_{2}-\theta_{1}\right)}{2 \lambda_{2} \gamma_{1}^{2}(t)}(\ln v)^{2}\right\}, & \text { if } u^{-\lambda_{2} \gamma_{1}(t)} \geq v^{-\lambda_{1} \gamma_{2}(t)} ; \\ u v \exp \left\{-\frac{\theta_{2}}{\gamma_{1}(t) \gamma_{2}(t)} \ln u \ln v\right\} \exp \left\{-\frac{\lambda_{2}\left(\theta_{1}-\theta_{2}\right)}{2 \lambda_{1} \gamma_{2}^{2}(t)}(\ln u)^{2}\right\}, & \text { if } u^{-\lambda_{2} \gamma_{1}(t)}<v^{-\lambda_{1} \gamma_{2}(t)},\end{cases}
$$

where $\gamma_{1}(t)=1+\lambda_{1} \theta_{2} t, \gamma_{2}(t)=1+\lambda_{2} \theta_{1}$ t and $u, v \in(0,1]$. Fix $a_{i}=\lambda_{1} \lambda_{2} \theta_{i}, i=1,2$ in (6.17) to verify that

$$
\bar{C}_{t}\left(\exp \left\{-\lambda_{1} x_{1}-\lambda_{1} \lambda_{2} \theta_{1} x_{1} t\right\}, \exp \left\{-\lambda_{2} x_{2}-\lambda_{1} \lambda_{2} \theta_{2} x_{2} t\right\}\right)=\frac{S_{X_{1}, X_{2}}\left(x_{1}+t, x_{2}+t\right)}{S_{X_{1}, X_{2}}(t, t)},
$$

for all $t \geq 0$. Therefore, the generalized Gumbel's bivariate exponential distribution given by (5.21) is member of the class $\mathcal{L}(\mathbf{x} ; \mathbf{a})$.

\subsection{Complementary patrimony of LS-BLMP}

We will consider in this section closure and reliability properties of the class $\mathcal{L}(\mathbf{x} ; \mathbf{a})$, or equivalently, of the survival functions possessing LS-BLMP.

\subsubsection{Closure properties}

The closure properties from Theorem 5.3.8 enable us to construct a wealth of bivariate distributions possessing LS-BLMP using as base a distribution having LS-BLMP, or equivalently, starting from a distribution belonging to the $\mathcal{L}(\mathbf{x} ; \mathbf{a})$ class defined by (5.6). Several consequences in terms of univariate failure rates are discussed below.

Remark 6.3.1. In Section 5.3 we suggested a procedure to generate distributions belonging to the class $\mathcal{L}(\mathbf{x} ; \mathbf{a})$ starting from given marginal failure rate functions. The construction scheme was then enriched by the closure properties listed in Theorem 5.3.8. Few comments are given below. 
- Relation (LS1) says that if $S_{Y_{1}, Y_{2}}\left(x_{1}, x_{2}\right)$ and $S_{Z_{1}, Z_{2}}\left(x_{1}, x_{2}\right)$ belong to $\mathcal{L}(\mathbf{x} ; \mathbf{D})$ then their product $S_{X_{1}, X_{2}}\left(x_{1}, x_{2}\right)=S_{Y_{1}, Y_{2}}\left(x_{1}, x_{2}\right) S_{Z_{1}, Z_{2}}\left(x_{1}, x_{2}\right) \in \mathcal{L}(\mathbf{x} ; \mathbf{D})$. Hence, the marginal failure rate function of $X_{1}$ is sum of the failure rates of $Y_{1}$ and $Z_{1}$ (analogously for $X_{2}$ ). Therefore, using $\left(Y_{1}, Y_{2}\right)$ and $\left(Z_{1}, Z_{2}\right)$ as a baseline distributions having $L S$-BLMP (constructed by procedure developed in Section 5.3), one will obtain the resulting vector $\left(X_{1}, X_{2}\right)$ which possesses LSBLMP, but with different constants $a_{i}, i=1,2$, in (6.6).

- Property (LS2) may be interpreted as follows. If $S_{Y_{1}, Y_{2}}\left(x_{1}, x_{2}\right)$ has $L S-B L M P$, then $S_{X_{1}, X_{2}}\left(x_{1}, x_{2}\right)=$ $\left[S_{Y_{1}, Y_{2}}\left(x_{1}, x_{2}\right)\right]^{c}$ will possess LS-BLMP for $c \geq 1$ and the vector $\left(X_{1}, X_{2}\right)$ may be constructed through marginal failure rate functions $r_{X_{1}}(x)=c r_{Y_{1}}(x)$ and $r_{X_{2}}(x)=c r_{Y_{2}}(x)$;

- The resulting marginal failure rate functions from (LS3) are given by

$$
r_{X_{1}}(x)=c_{1} r_{Y_{1}}(x)+c_{1} r_{Z_{1}}(x) \quad \text { and } \quad r_{X_{2}}(x)=c_{1} r_{Y_{2}}(x)+c_{2} r_{Z_{2}}(x)
$$

and one would be able to generate a bivariate distribution of $\left(X_{1}, X_{2}\right)$ having LS-BLMP;

- Following (LS4) the corresponding marginal failure rate functions are $r_{X_{1}}(x)=\alpha r_{Y_{1}}(\alpha x)$ and $r_{X_{2}}(x)=\alpha r_{Y_{2}}(\alpha x)$.

\subsubsection{Reliability properties}

As mentioned in Section 6.1, the function $B\left(x_{1}, x_{2}, t\right)$ introduces a specific aging effect on distributions belonging to the class $\mathcal{L}(\mathbf{x} ; \mathbf{a})$. Here we will present few reliability properties of the class $\mathcal{L}(\mathbf{x} ; \mathbf{a})$. Initially consider the following definition.

Definition 6.3.2 (Brindley and Thompson (1972)). A survival function defined in the positive quadrant is Bivariate Increasing Failure Rate (BIFR) if the conditional probability $\mathbb{P}\left(X_{1}>x_{1}+\right.$ $\left.t, X_{2}>x_{2}+t \mid X_{1}>x_{1}, X_{2}>x_{2}\right)$ is decreasing in $x_{1}, x_{2}$ for all $t>0$. It is called Bivariate Decreasing Failure Rate (BDFR) if the last conditional probability exhibits an increasing behaviour.

Therefore all bivariate distributions that satisfy (6.3), e.g. that possess LS-BLMP, are BIFR according to Definition 6.3.2.

The following statement is true.

Lemma 6.3.3. If $S_{X_{1}, X_{2}}\left(x_{1}, x_{2}\right)$ satisfies (6.3) then $X=\min \left(X_{1}, X_{2}\right)$ is increasing failure rate.

Proof. Let $S_{X}(t)=\mathbb{P}\left(\min \left(X_{1}, X_{2}\right)>t\right)$. From Theorem 6.2.9, we have (6.3) equivalent to (5.6). Since $S_{X}(t)=S_{X_{1}, X_{2}}(t, t)$, from (5.6), $S_{X_{1}, X_{2}}(t, t)=\exp \left\{-a_{0} t-\frac{a_{1}+a_{2}}{2} t^{2}\right\}$, which is IFR.

Note that if $a_{1}=a_{2}=0$ we have the $\mathrm{BLMP}_{1}$ and $X=\min \left(X_{1}, X_{2}\right)$ has constant failure rate, i.e. it is exponentially distributed.

The next results are related with bivariate increasing hazard rate (BIHR) and bivariate decreasing hazard rate (BDHR) definitions of Johnson and Kotz (1975), see Definition 3.6.21.

Lemma 6.3.4. Suppose $S_{X_{1}, X_{2}}\left(x_{1}, x_{2}\right)$ satisfies (6.3). If $a_{1}=a_{2}=a>0$ and $X_{1}$ and $X_{2}$ are univariate IFR then $S_{X_{1}, X_{2}}\left(x_{1}, x_{2}\right)$ is BIHR. 
Proof. Let us first analyse $r_{1}\left(x_{1}, x_{2}\right)$. From Theorem 6.2.9, we have (6.3) equivalent to (5.6). Thus, imposing the condition $a_{1}=a_{2}=a$ in the expression for $r_{1}\left(x_{1}, x_{2}\right)$ given in (5.9) we obtain

$$
r_{1}\left(x_{1}, x_{2}\right)= \begin{cases}a_{0}+a x_{2}-r_{X_{2}}\left(x_{2}-x_{1}\right), & \text { if } 0 \leq x_{1}<x_{2} \\ r_{X_{1}}\left(x_{1}-x_{2}\right)+a x_{2}, & \text { if } x_{1}>x_{2} \geq 0\end{cases}
$$

Clearly, $r_{1}\left(x_{1}, x_{2}\right)$ is increasing in $x_{1}$ in both regions $\left\{0 \leq x_{1}<x_{2}\right\}$ and $\left\{x_{1}>x_{2} \geq 0\right\}$.

Let us analyse $r_{1}\left(x_{1}, x_{2}\right)$ when $x_{1}=x_{2}=x \geq 0$. First, suppose $S_{X_{1}, X_{2}}\left(x_{1}, x_{2}\right)$ is absolutely continuous, i.e. $a_{0}=f_{X_{1}}(0)+f_{X_{2}}(0)=r_{X_{1}}(0)+r_{X_{2}}(0)$. Thus, $r_{1}\left(x_{1}, x_{2}\right)$ is continuous at $\left(x_{1}, x_{2}\right)=$ $(x, x)$, which implies

$$
r_{1}(x, x)=a_{0}+a x-r_{X_{2}}(0)=r_{X_{1}}(0)+a x .
$$

Therefore, $r_{1}\left(x_{1}, x_{2}\right)$ is increasing in $x_{1}$ for all $x_{1}, x_{2} \geq 0$. Similar conclusion holds for $r_{2}\left(x_{1}, x_{2}\right)$.

Note that in the absolutely continuous case we have

$$
\lim _{x_{1} \rightarrow x_{2}^{-}} r_{i}\left(x_{1}, x_{2}\right)=\lim _{x_{1} \rightarrow x_{2}^{+}} r_{i}\left(x_{1}, x_{2}\right), \quad i=1,2 .
$$

If $S_{X_{1}, X_{2}}\left(x_{1}, x_{2}\right)$ is not absolutely continuous, $r_{1}\left(x_{1}, x_{2}\right)$ and $r_{2}\left(x_{1}, x_{2}\right)$ are not defined when $x_{1}=x_{2}=x \geq 0$ but one can still consider the limits

$$
\lim _{x_{1} \rightarrow x_{2}^{-}} r_{1}\left(x_{1}, x_{2}\right) \quad \text { and } \lim _{x_{1} \rightarrow x_{2}^{+}} r_{1}\left(x_{1}, x_{2}\right) .
$$

For bivariate distributions with singular part we have $a_{0}<r_{X_{1}}(0)+r_{X_{2}}(0)$, so we get the inequality

$$
\lim _{x_{1} \rightarrow x_{2}^{-}} r_{1}\left(x_{1}, x_{2}\right)<\lim _{x_{1} \rightarrow x_{2}^{+}} r_{1}\left(x_{1}, x_{2}\right) .
$$

Thus, $r_{1}\left(x_{1}, x_{2}\right)$ exhibits an upward jump at points $\left(x_{1}, x_{2}\right)=(x, x)$. Consequently, is increasing in $x_{1}$ for all $x_{1}, x_{2} \geq 0$.

Analogously one can verify that $r_{2}\left(x_{1}, x_{2}\right)$ realizes a jump at points $\left(x_{1}, x_{2}\right)=(x, x)$, i.e. it is increasing in $x_{2}$ for all $x_{1}, x_{2} \geq 0$.

Remark 6.3.5. Consider the proof of Lemma 6.3.4 and let $T_{i}$ be independent exponentially distributed with parameter $\lambda_{i}$ in stochastic representation (2.9). Then, expression (6.20) for bivariate MO exponential distribution takes the form

$$
r_{i}\left(x_{1}, x_{2}\right)= \begin{cases}\lambda_{i}, & \text { if } 0 \leq x_{i}<x_{3-i} \\ \lambda_{i}+\lambda_{3}, & \text { if } x_{i}>x_{3-i} \geq 0\end{cases}
$$

for $i=1,2$. This example is just an illustration of the fact that when $a_{1}=a_{2}=a, X_{i}$ is IFR and in the presence of singularity $r_{i}\left(x_{1}, x_{2}\right), i=1,2$, is increasing in $x_{1}$, with a jump at $x_{1}=x_{2}=x \geq 0$. In this example we have $a=0$.

Remark 6.3.6. If $X_{1}$ and $X_{2}$ have constant failure rates, e.g. $r_{X_{1}}(x)=c_{1}$ and $r_{X_{2}}(x)=c_{2}$, then $r_{1}\left(x_{1}, x_{2}\right)$ and $r_{2}\left(x_{1}, x_{2}\right)$ given by (6.20) are locally constants under conditions of Lemma 6.3.4. So, we obtain the Gumbel's bivariate exponential distribution given by (5.3) after imposing absolutely continuity to $S_{X_{1}, X_{2}}\left(x_{1}, x_{2}\right)$. Without imposing absolutely continuity, one would get a distribution 
with singularity along the line $x_{1}=x_{2}=x \geq 0$ such that the two components of the hazard gradient vector $r_{1}\left(x_{1}, x_{2}\right)$ and $r_{2}\left(x_{1}, x_{2}\right)$ will be locally constants as well, see the survival function given by (5.23) and Remark 5.3.11, as well.

In the proof of Lemma 6.3.4 we got that

$$
\lim _{x_{1} \rightarrow x_{2}^{-}} r_{1}\left(x_{1}, x_{2}\right) \leq \lim _{x_{1} \rightarrow x_{2}^{+}} r_{1}\left(x_{1}, x_{2}\right)
$$

with equality only in the case of absolute continuity. An analogous relation is valid for $r_{2}\left(x_{1}, x_{2}\right)$. Therefore, one can obtain a similar condition $S_{X_{1}, X_{2}}\left(x_{1}, x_{2}\right)$ to be BDHR under absolute continuity assumption as stated in the next lemma.

Lemma 6.3.7. Suppose $S_{X_{1}, X_{2}}\left(x_{1}, x_{2}\right)$ satisfies (6.3) with $a_{1}=a_{2}$. If $X_{1}$ and $X_{2}$ are univariate DFR and $S_{X_{1}, X_{2}}\left(x_{1}, x_{2}\right)$ is absolutely continuous, then $S_{X_{1}, X_{2}}\left(x_{1}, x_{2}\right)$ is BDHR.

Proof. Follows the same steps of Lemma 6.3.4.

When $a_{1} \neq a_{2}$ in (6.3) we have the following version.

Lemma 6.3.8. Suppose $S_{X_{1}, X_{2}}\left(x_{1}, x_{2}\right)$ satisfies (6.3). If $a_{1}>a_{2}$ (or $a_{2}>a_{1}$ ) and $X_{1}$ and $X_{2}$ are univariate IFR such that

$$
\frac{d}{d x} r_{X_{1}}(x) \geq a_{1}-a_{2} \quad\left(\text { or } \quad \frac{d}{d x} r_{X_{2}}(x) \geq a_{2}-a_{1}\right) .
$$

Then, $S_{X_{1}, X_{2}}\left(x_{1}, x_{2}\right)$ is BIHR.

Proof. Suppose $a_{1}>a_{2}$. From Theorem 6.2.9, we have (6.3) equivalent to (5.6). Thus, from (5.9), $r_{1}\left(x_{1}, x_{2}\right)$ is an increasing function of $x_{1}$ for all $x_{1}, x_{2} \geq 0$. In particular,

$$
\lim _{x_{1} \rightarrow x_{2}^{-}} r_{1}\left(x_{1}, x_{2}\right) \leq \lim _{x_{1} \rightarrow x_{2}^{+}} r_{1}\left(x_{1}, x_{2}\right) .
$$

From the expression for $r_{2}\left(x_{1}, x_{2}\right)$ given in (5.10) we have

$$
r_{2}\left(x_{1}, x_{2}\right)= \begin{cases}a_{0}+a_{1} x_{1}+\left(a_{2}-a_{1}\right) x_{2}-r_{X_{1}}\left(x_{1}-x_{2}\right), & \text { if } 0 \leq x_{2}<x_{1}, \\ r_{X_{2}}\left(x_{2}-x_{1}\right)+a_{2} x_{1}, & \text { if } x_{2}>x_{1} \geq 0 .\end{cases}
$$

When $0 \leq x_{2}<x_{1}$ under the condition $\frac{d}{d x} r_{X_{1}}(x) \geq a_{1}-a_{2}$, we conclude that $r_{2}\left(x_{1}, x_{2}\right)$ is increasing in $x_{2}$. If $x_{2}>x_{1} \geq 0$, we similarly conclude that $r_{2}\left(x_{1}, x_{2}\right)$ is increasing in $x_{2}$ if $X_{2}$ is IFR. Moreover

$$
\lim _{x_{2} \rightarrow x_{1}^{-}} r_{2}\left(x_{1}, x_{2}\right) \leq \lim _{x_{2} \rightarrow x_{1}^{+}} r_{2}\left(x_{1}, x_{2}\right)
$$

and $r_{2}\left(x_{1}, x_{2}\right)$ is an increasing function of $x_{2}$ for all $x_{1}, x_{2} \geq 0$.

The proof is analogous if we assume $a_{2}>a_{1}$ and $\frac{d}{d x} r_{X_{2}}(x) \geq a_{2}-a_{1}$.

\subsection{Concluding remarks}

In this chapter a new notion called "Linear Sibuya-type of bivariate lack-of-memory property" (LS-BLMP) was introduced and its usefulness for explaining the aging phenomena pointed out. 
The set of bivariate distributions possessing LS-BLMP is rich: it is composed by continuous distributions that are $\mathrm{BLMP}_{1}$ or $\mathrm{BLMP}_{2}$; symmetric or asymmetric; $\mathrm{PQD}$ or NQD; absolutely continuous or having a singular component. None of these distributional subsets are related to each other. Therefore, starting the modeling process with a flexible family of distributions with enough members (as LS-BLMP is) increases the chances to accommodate different data situations.

Our motivation was to link the two classical BLMP versions $\left(\mathrm{BLMP}_{1}\right.$ and $\left.\mathrm{BLMP}_{2}\right)$ into a more general one. The key idea was to preserve the dependence function $H_{X_{1}, X_{2}}\left(x_{1}, x_{2}\right)$ specified by (3.4) for the residual lifetime vector $\mathbf{X}_{t}$ and the original one $\mathbf{X}=\left(X_{1}, X_{2}\right)$, independent of time $t$. Notice that the preservation of the dependence function is a weaker condition than the preservation of both the dependence function and marginals, as required under BLMP 1 .

In Definition 6.2.1 of the LS-BLMP we additionally impose natural aging conditions on marginals, see corresponding expression (6.6). This new definition of BLMP is not limited to exchangeability, which is a desirable feature since the symmetry model becomes more and more restricted in large dimensions.

It happens that LS-BLMP is characterized by relation (6.3), see Theorem 6.2.4. Therefore, the LS-BLMP is an appropriate candidate to describe the aging phenomena, offering a model (6.3) as a counterpart and complement to the virtual "non-aging" BLMP ${ }_{1}$ world represented by (2.7). The additional nonnegative parameters $a_{1}$ and $a_{2}$ enter naturally as intensities of homogeneous Poisson processes causing individual shocks and provide flexibility with respect to modeling.

The LS-BLMP has an important interpretation related with the hazard vector fields known in classical physics. It was proved in Theorem 6.2.9 that LS-BLMP is characterized by the class $\mathcal{L}(\mathbf{x} ; \mathbf{a})$, introduced in Chapter 5 and composed by bivariate continuous distributions such that the sum of the components of hazard gradient vector is a linear function of both arguments $x_{1}$ and $x_{2}$, i.e. satisfy (5.2).

From the point of view of distribution theory, we provided a method for constructing a wealth of bivariate distributions possessing LS-BLMP starting from another distribution belonging to the class $\mathcal{L}(\mathbf{x} ; \mathbf{a})$, see Section 6.3.

A multivariate version of LS-BLMP can also be introduced. We did not consider inference procedures related to LS-BLMP, nor application for real data set. This important issue is part of our future lines of research. We do believe that the new bivariate memory-less notion will be both of theoretical and practical interest. 


\section{Chapter 7}

\section{Sibuya Type Bivariate Lack-of-memory Property}

\subsection{Introduction}

In Chapter 5, Definition 5.2.1 introduced the class $\mathcal{L}(\mathbf{x} ; \mathbf{a})$ of nonnegative bivariate continuous distributions such that the sum of the components of hazard gradient vector is a linear function of both arguments $x_{1}$ and $x_{2}$ and Theorem 5.2.11 presented the general form of the corresponding survival function. In Chapter 6, Definition 6.2.1 introduced the linear Sibuya BLMP and Theorem 6.2.9 showed the class $\mathcal{L}(\mathbf{x} ; \mathbf{a})$ can be characterized by the LS-BLMP.

Remark 7.1.1 (Restriction on the distributions). Because of this important equivalence between $L S-B L M P$ and the class $\mathcal{L}(\mathbf{x} ; \mathbf{a})$, it will be assumed hereafter that $S_{X_{1}, X_{2}}\left(x_{1}, x_{2}\right)$ is decomposable into an absolutely continuous component, with support on $\mathbf{R}_{+}^{2}$, and a singular one, with support on the set $\Omega$ given in (2.10),

$$
\Omega=\left\{\left(x_{1}, x_{2}\right) \in \mathbf{R}_{+}^{2} \mid x_{1}=x_{2}=x\right\} .
$$

Whenever we make use of such equivalence, we suppose the continuous survival function $S_{X_{1}, X_{2}}\left(x_{1}, x_{2}\right)$ has continuous first partial derivatives, with the possibility of nonexistence on the set $\Omega$. See also the related Remarks 5.2.2, 5.2.7, 5.2.10 and 7.3.10.

According with Definition 6.2.1, the nonnegative continuous random vector $\left(X_{1}, X_{2}\right)$ possesses the linear Sibuya BLMP if and only if conditions (6.5) and (6.6), i.e.,

$$
\begin{gathered}
\frac{S_{\mathbf{X}_{t}}\left(x_{1}, x_{2}\right)}{S_{X_{1 t}}\left(x_{1}\right) S_{X_{2 t}}\left(x_{2}\right)}=\frac{S_{X_{1}, X_{2}}\left(x_{1}, x_{2}\right)}{S_{X_{1}}\left(x_{1}\right) S_{X_{2}}\left(x_{2}\right)} \quad \text { for all } \quad x_{1}, x_{2} \geq 0, t \geq 0 \quad \text { and } \\
S_{X_{i t}}\left(x_{i}\right)=S_{X_{i}}\left(x_{i}\right) \exp \left\{-a_{i} x_{i} t\right\} \quad \text { for } \quad a_{i}>0, i=1,2 .
\end{gathered}
$$

hold true, where $S_{X_{1 t}}\left(x_{1}\right)$ and $S_{X_{2 t}}\left(x_{2}\right)$ are the marginal survival functions of residual lifetime vector $\mathbf{X}_{t}$ corresponding to $\left(X_{1}, X_{2}\right)$.

It is direct to check that LS-BLMP transforms into BLMP when $a_{1}=a_{2}=0$ in relation (5.2) which defines the class $\mathcal{L}(\mathbf{x} ; \mathbf{a})$. Really, for continuous bivariate distributions with $\mathrm{BLMP}_{1}$, we always have $r_{1}\left(x_{1}, x_{2}\right)+r_{2}\left(x_{1}, x_{2}\right)=a_{0}$, see Theorem 2 in Kulkarni (2006).

Since many bivariate distributions possess $\mathrm{BLMP}_{1}$ when relaxing the assumption of marginals exponentially distributed, it would be fruitful to ignore condition (6.6) in Definition 6.2.1 of LS- 
BLMP and obtain the class of distributions characterized by this weaker property, to be named Sibuya BLMP in Definition 7.2.1. Of course, this larger class, to be denoted by $\mathcal{L}(\mathbf{x} ; \mathbf{A})$, will contain all members of $\mathcal{L}(\mathbf{x} ; \mathbf{a})$.

In this chapter we find the functional form for the sum $r_{1}\left(x_{1}, x_{2}\right)+r_{2}\left(x_{1}, x_{2}\right)$ when only requiring relation (6.5) to be satisfied, i.e. in the case of memory-less dependence function $H_{X_{1}, X_{2}}\left(x_{1}, x_{2}\right)$. In Section 7.2 we define the Sibuya BLMP based on relation (6.5), establish a characterizing functional equation in terms of dependence function $H_{X_{1}, X_{2}}\left(x_{1}, x_{2}\right)$ and, considering the Generalized Marshall-Olkin (GMO) models studied by Li and Pellerey (2011), provide an indication for the decomposition of the sum $r_{1}\left(x_{1}, x_{2}\right)+r_{2}\left(x_{1}, x_{2}\right)$ we are looking for.

In Section 7.3 we introduce the extended class $\mathcal{L}(\mathbf{x} ; \mathbf{A})$ and show its equivalence with the class of distributions characterized by Sibuya BLMP. Several characterizations of $\mathcal{L}(\mathbf{x} ; \mathbf{A})$ are also obtained and illustrated with examples. In Section 7.4 we present the closure properties of the extended class and suggest a general building procedure to generate its members. Non-aging survival copulas are briefly considered in Section 7.5. In Section 7.6 we offer a stronger version of S-BLMP. Conclusions are presented in Section 7.7.

\subsection{Weaker version of the linear Sibuya-BLMP}

In the first part of this section we define a weaker form of LS-BLMP and characterize the distributions with this property via a functional equation involving the dependence function $H_{X_{1}, X_{2}}\left(x_{1}, x_{2}\right)$. In the second part we find under which conditions the GMO distributions satisfy the introduced property. This will help us to define the corresponding extended class $\mathcal{L}(\mathbf{x} ; \mathbf{A})$ in Section 7.3.

In what follows, we consider nonnegative bivariate continuous distributions that, besides condition stated at Remark 7.1.1, satisfy the weaker form of LS-BLMP as postulated by the following

Definition 7.2.1. The nonnegative continuous bivariate distribution $\left(X_{1}, X_{2}\right)$ possesses Sibuya $B L M P$ (to be abbreviated $S$-BLMP) if and only if relation (6.5)

$$
\frac{S_{\mathbf{X}_{t}}\left(x_{1}, x_{2}\right)}{S_{X_{1 t}}\left(x_{1}\right) S_{X_{2 t}}\left(x_{2}\right)}=\frac{S_{X_{1}, X_{2}}\left(x_{1}, x_{2}\right)}{S_{X_{1}}\left(x_{1}\right) S_{X_{2}}\left(x_{2}\right)}
$$

is satisfied for all $x_{1}, x_{2} \geq 0$ and $t \geq 0$.

Definition 7.2.1 can be interpreted as follows: the baseline vector $\left(X_{1}, X_{2}\right)$ and its residual life time vector $\mathbf{X}_{t}$, given by relation (3.13), should share the same dependence function $H_{X_{1}, X_{2}}\left(x_{1}, x_{2}\right)$, defined by relation (3.4), which does not depend on $t \geq 0$. This new concept includes as a special case the preservation of joint distributions of $\left(X_{1}, X_{2}\right)$ and corresponding $\mathbf{X}_{t}$, as advocated by BLMP.

\subsubsection{A characterizing functional equation}

Remind that the marginal survival functions of residual lifetime vector $\mathbf{X}_{t}$ are given by

$$
S_{X_{1 t}}\left(x_{1}\right)=\frac{S_{X_{1}, X_{2}}\left(x_{1}+t, t\right)}{S_{X_{1}, X_{2}}(t, t)} \quad \text { and } \quad S_{X_{2 t}}\left(x_{2}\right)=\frac{S_{X_{1}, X_{2}}\left(t, x_{2}+t\right)}{S_{X_{1}, X_{2}}(t, t)}
$$


see relation (6.4). Moreover, from Section 6.2, condition (6.5) from Definition 7.2.1 is equivalent to (6.8), given by

$$
\frac{S_{X_{1}, X_{2}}\left(x_{1}+t, x_{2}+t\right)}{S_{X_{1}, X_{2}}\left(x_{1}+t, t\right) S_{X_{1}, X_{2}}\left(t, x_{2}+t\right)}=\frac{S_{X_{1}, X_{2}}\left(x_{1}, x_{2}\right)}{S_{X_{1}}\left(x_{1}\right) S_{X_{2}}\left(x_{2}\right) S_{X_{1}, X_{2}}(t, t)} .
$$

The following characterization is valid.

Lemma 7.2.2. The $S-B L M P$ is characterized by the functional equation

$$
\begin{aligned}
H_{X_{1}, X_{2}}\left(x_{1}+t, x_{2}+t\right) & =H_{X_{1}, X_{2}}\left(x_{1}+t, t\right)+H_{X_{1}, X_{2}}\left(t, x_{2}+t\right) \\
& +H_{X_{1}, X_{2}}\left(x_{1}, x_{2}\right)-H_{X_{1}, X_{2}}(t, t),
\end{aligned}
$$

which has to be fulfilled for all $x_{1}, x_{2}, t \geq 0$. The solution has to satisfy the inequalities

$$
\begin{gathered}
\max \left[0, \exp \left\{H_{X_{1}}\left(x_{1}\right)\right\}+\exp \left\{H_{X_{2}}\left(x_{2}\right)\right\}-\exp \left\{H_{X_{1}}\left(x_{1}\right)+H_{X_{2}}\left(x_{2}\right)\right\}\right] \leq \exp \left\{H_{X_{1}, X_{2}}\left(x_{1}, x_{2}\right)\right\} \\
\leq \min \left[\exp \left\{H_{X_{1}}\left(x_{1}\right)\right\}, \exp \left\{H_{X_{2}}\left(x_{2}\right)\right\}\right]
\end{gathered}
$$

where $H_{X_{i}}\left(x_{i}\right)$ are the marginal cumulative failure rates, $i=1,2$.

Proof. Apply the exponential representation (3.3) in (6.8) to get the functional equation (7.1). Inversely, use the defining relation for dependence function given by (3.4) in (7.1) to recover (6.8).

Notice that equation (7.1) does not involve the marginal cumulative failure rates and one can obtain solutions that do not generate a proper survival function $S_{X_{1}, X_{2}}\left(x_{1}, x_{2}\right)$ given in its exponential representation form by (3.3). Hence, restrictions on $H_{X_{1}, X_{2}}\left(x_{1}, x_{2}\right)$ are needed.

It is easy to deduce that applying the usual Fréchet-Hoeffding bounds given by (3.7) to $S_{X_{1}, X_{2}}\left(x_{1}, x_{2}\right)$ we get

$$
\max \left\{0, \frac{S_{X_{1}}\left(x_{1}\right)+S_{X_{2}}\left(x_{2}\right)-1}{S_{X_{1}}\left(x_{1}\right) S_{X_{2}}\left(x_{2}\right)}\right\} \leq \frac{S_{X_{1}, X_{2}}\left(x_{1}, x_{2}\right)}{S_{X_{1}}\left(x_{1}\right) S_{X_{2}}\left(x_{2}\right)} \leq \min \left\{\frac{1}{S_{X_{1}}\left(x_{1}\right)}, \frac{1}{S_{X_{2}}\left(x_{2}\right)}\right\} .
$$

One can obtain the inequalities in Lemma 7.2.2 using the definition of dependence function given by relation (3.4) and $\frac{1}{S_{X_{i}}\left(x_{i}\right)}=\exp \left\{H_{X_{i}}\left(x_{i}\right)\right\}, i=1,2$.

Remark 7.2.3. In Section 3.6 we obtained relation (7.1) as the functional equation (3.23) and used it to establish BLMP 1 in EMO models.

The trivial solution of the functional equation (7.1) is $H_{X_{1}, X_{2}}\left(x_{1}, x_{2}\right)=0$, which characterizes the independence between nonnegative continuous random variables $X_{1}$ and $X_{2}$. In fact, if $X_{1}$ and $X_{2}$ are independent, so are the components of the corresponding residual lifetime vector $\mathbf{X}_{t}$.

As a direct consequence of Lemma 7.2.2 we have the following

Corollary 7.2.4. Let $H_{Y_{1}, Y_{2}}\left(x_{1}, x_{2}\right)$ and $H_{Z_{1}, Z_{2}}\left(x_{1}, x_{2}\right)$ be two different solutions of the functional equation (7.1). Then, any linear combination of these solutions is also a solution of (7.1).

The next example illustrates an application of Corollary 7.2.4.

Example 7.2.5 (Revisiting Example 5.3.10). Let the bivariate random vector $\left(T_{1}, T_{2}\right)$ follow the absolutely continuous Gumbel's type I bivariate exponential distribution with survival function given 
by (5.3)

$$
S_{T_{1}, T_{2}}\left(x_{1}, x_{2}\right)=\exp \left\{-\lambda_{1} x_{1}-\lambda_{2} x_{2}-\theta \lambda_{1} \lambda_{2} x_{1} x_{2}\right\}, \quad \theta \in[0,1], \lambda_{1}, \lambda_{2}>0 .
$$

Its dependence function is $H_{T_{1}, T_{2}}\left(x_{1}, x_{2}\right)=-\theta \lambda_{1} \lambda_{2} x_{1} x_{2}$ and satisfies the functional equation (7.1), i.e. the distribution possesses S-BLMP. Let $T_{3}$ be exponentially distributed with parameter $\lambda_{3}>0$ and independent of $\left(T_{1}, T_{2}\right)$. Applying the stochastic representation (2.9), the resulting joint survival function for $\left(X_{1}, X_{2}\right)$ is given by equation (5.23), i.e.,

$$
S_{X_{1}, X_{2}}\left(x_{1}, x_{2}\right)= \begin{cases}\exp \left\{-\left(\lambda_{1}+\lambda_{3}\right) x_{1}-\lambda_{2} x_{2}-\theta \lambda_{1} \lambda_{2} x_{1} x_{2}\right\}, & \text { if } x_{1} \geq x_{2} \geq 0, \\ \exp \left\{-\lambda_{1} x_{1}-\left(\lambda_{2}+\lambda_{3}\right) x_{2}-\theta \lambda_{1} \lambda_{2} x_{1} x_{2}\right\}, & \text { if } x_{2} \geq x_{1} \geq 0 .\end{cases}
$$

This survival function has absolutely continuous and singular parts, the last one with support on the set $\Omega$ given by (2.10). Its dependence function is given by

$$
H_{X_{1}, X_{2}}\left(x_{1}, x_{2}\right)=\lambda_{3} \min \left(x_{1}, x_{2}\right)-\theta \lambda_{1} \lambda_{2} x_{1} x_{2},
$$

which is a linear combination of dependence functions that solve the functional equation (7.1). Therefore, according with Corollary 7.2.4, the distribution given by (5.23) possesses S-BLMP.

In the next example, where we consider a stochastic representation different from (2.9), Corollary 7.2.4 can be applied as well.

Example 7.2.6 (A stochastic representation generating S-BLMP distributions). Let $\left(Y_{1}, Y_{2}\right)$ and $\left(Z_{1}, Z_{2}\right)$ be two independent nonnegative continuous random vectors. Suppose that their dependence functions $H_{Y_{1}, Y_{2}}\left(x_{1}, x_{2}\right)$ and $H_{Z_{1}, Z_{2}}\left(x_{1}, x_{2}\right)$ satisfy the functional equation (7.1). Consider the stochastic representation

$$
\left(X_{1}, X_{2}\right)=\left(\min \left\{Y_{1}, Z_{1}\right\}, \min \left\{Y_{2}, Z_{2}\right\}\right)
$$

and notice that

$$
S_{X_{1}, X_{2}}\left(x_{1}, x_{2}\right)=P\left(Y_{1}>x_{1}, Z_{1}>x_{1}, Y_{2}>x_{2}, Z_{2}>x_{2}\right)=S_{Y_{1}, Y_{2}}\left(x_{1}, x_{2}\right) S_{Z_{1}, Z_{2}}\left(x_{1}, x_{2}\right) .
$$

Therefore, $H_{X_{1}, X_{2}}\left(x_{1}, x_{2}\right)=H_{Y_{1}, Y_{2}}\left(x_{1}, x_{2}\right)+H_{Z_{1}, Z_{2}}\left(x_{1}, x_{2}\right)$. According with Corollary 7.2.4, $S_{X_{1}, X_{2}}\left(x_{1}, x_{2}\right)$ possesses $S$-BLMP.

In what follows, we find under which conditions the GMO distributions satisfy the S-BLMP introduced in Definition 7.2.1. This will help us to define the corresponding extended class of bivariate distributions in Section 7.3.

\subsubsection{Related GMO models}

Let us consider the GMO models introduced by Li and Pellerey (2011), with joint survival function given by (3.1). GMO models satisfy the stochastic representation (2.9) but the independent nonnegative absolutely continuous random variables $T_{i}$ are not necessarily exponentially distributed, $i=1,2,3$.

Since GMO models satisfy the functional equation (2.6) only in the case of exponentially distributed $T_{i}, i=1,2,3$, in general such models do not possess BLMP 1 . But in some special cases 
GMO distributions follow LS-BLMP: for example see the survival function given by (5.5) and Example 7.3.14. Notice that GMO models exhibit a singular component with support on the set $\Omega$ given by (2.10), so they are not absolutely continuous.

Let us verify under which conditions the GMO models fulfill (6.5), i.e., we are interested when GMO distributions possess S-BLMP. The answer is given in the following lemma.

Lemma 7.2.7. The GMO distributions (3.1) follow $S$-BLMP if and only if $T_{3}$ is exponentially distributed.

Proof. The dependence function $H_{X_{1}, X_{2}}\left(x_{1}, x_{2}\right)$ of GMO models (3.1) is given by

$$
H_{X_{1}, X_{2}}\left(x_{1}, x_{2}\right)=\ln \left\{\frac{S_{X_{1}, X_{2}}\left(x_{1}, x_{2}\right)}{S_{X_{1}}\left(x_{1}\right) S_{X_{2}}\left(x_{2}\right)}\right\}=H_{T_{3}}\left(\min \left\{x_{1}, x_{2}\right\}\right),
$$

see defining relation (3.4).

Let the GMO distribution follow S-BLMP. From Lemma 7.2.2, the functional equation (7.1) holds true. Initially suppose that $x_{1} \geq x_{2} \geq 0$. Substitute (7.3) in (7.1) to obtain the Cauchy functional equation

$$
H_{T_{3}}\left(x_{2}+t\right)=H_{T_{3}}\left(x_{2}\right)+H_{T_{3}}(t) \text { for all } \quad x_{2}, t \geq 0
$$

with only continuous solution $H_{T_{3}}(x)=a_{0} x$ for some constant $a_{0}$. Since $H_{T_{3}}(x) \geq 0$ for all $x \geq 0$, we have $a_{0} \geq 0$. The same is the conclusion when $x_{2} \geq x_{1} \geq 0$. This means that $T_{3}$ is exponentially distributed with parameter $a_{0}$.

To finish the proof, observe that if $T_{3}$ is exponentially distributed with parameter $a_{0}$ in (3.1), from (7.3) we have the dependence function $H_{X_{1}, X_{2}}\left(x_{1}, x_{2}\right)=a_{0} \min \left\{x_{1}, x_{2}\right\}$ and the functional equation (7.1) is fulfilled.

From expression (7.3), Lemma 7.2.7 and relations $r_{i}\left(x_{1}, x_{2}\right)=-\frac{\partial}{\partial x_{i}} \ln S_{X_{1}, X_{2}}\left(x_{1}, x_{2}\right), i=1,2$, one arrives to the following

Lemma 7.2.8. The GMO distributions with survival function given by (3.1) follow $S$-BLMP if and only if

$$
r_{1}\left(x_{1}, x_{2}\right)+r_{2}\left(x_{1}, x_{2}\right)=a_{0}+r_{T_{1}}\left(x_{1}\right)+r_{T_{2}}\left(x_{2}\right), \quad a_{0}>0
$$

for all $x_{1}, x_{2} \geq 0$, where $r_{T_{i}}(x)$ are the failure rate functions of random variables $T_{i}, i=1,2$.

Therefore, the sum of the components of the hazard gradient vector of GMO models can be represented as a sum of a constant and two continuous integrable functions of $x_{i}$, being marginal failure rate functions $r_{T_{i}}\left(x_{i}\right), i=1,2$.

\subsection{Characterizations of S-BLMP}

Based on the additive decomposition of the sum $r_{1}\left(x_{1}, x_{2}\right)+r_{2}\left(x_{1}, x_{2}\right)$ obtained in Lemma 7.2.8 for GMO models, in the first part of this section we extend the class $\mathcal{L}(\mathbf{x} ; \mathbf{a})$, characterized in Chapter 5 by relation (5.2), and denote it by $\mathcal{L}(\mathbf{x} ; \mathbf{A})$. We also obtain the general expression of the survival function for the members of $\mathcal{L}(\mathbf{x} ; \mathbf{A})$. In the second part, we show the equivalence between the class $\mathcal{L}(\mathbf{x} ; \mathbf{A})$ and the distributions possessing S-BLMP. In the third part, we provide a 
stochastic representation for the members of $\mathcal{L}(\mathbf{x} ; \mathbf{A})$. We close this section presenting a condition that characterizes when a member of $\mathcal{L}(\mathbf{x} ; \mathbf{A})$ is absolutely continuous or, additionally, has a singular component with support on the set $\Omega$ given by (2.10).

\subsubsection{Definition and characterization of the extended class $\mathcal{L}(\mathrm{x} ; \mathrm{A})$}

The representation for the sum of the hazard gradient vector components obtained in Lemma 7.2.8 characterizes the GMO distributions possessing S-BLMP. That particular additive decomposition for $r\left(x_{1}, x_{2}\right)$ is not restricted to GMO models. Observe that, from Section 6.2, the characterizing relation (6.5) is equivalent to (6.8), which can be alternatively presented as

$$
S_{X_{1}, X_{2}}\left(x_{1}+t, x_{2}+t\right)=S_{X_{1}, X_{2}}\left(x_{1}, x_{2}\right) S_{X_{1}, X_{2}}(t, t) Q_{1}\left(x_{1}, t\right) Q_{2}\left(x_{2}, t\right),
$$

where the functions $Q_{i}\left(x_{i}, t\right), i=1,2$, are given by

$$
Q_{1}\left(x_{1}, t\right)=\frac{S_{X_{1}, X_{2}}\left(x_{1}+t, t\right)}{S_{X_{1}, X_{2}}(t, t) S_{X_{1}}\left(x_{1}\right)} \quad \text { and } \quad Q_{2}\left(x_{2}, t\right)=\frac{S_{X_{1}, X_{2}}\left(t, x_{2}+t\right)}{S_{X_{1}, X_{2}}(t, t) S_{X_{2}}\left(x_{2}\right)},
$$

and are such that $Q_{i}(0, t)=Q_{i}\left(x_{i}, 0\right)=1, i=1,2$. Notice that $Q_{i}\left(x_{i}, t\right)=\frac{S_{X_{i t}}\left(x_{i}\right)}{S_{X_{i}}\left(x_{i}\right)}, i=1,2$.

According with Remark 5.2.4, if $S_{X_{1}, X_{2}}\left(x_{1}, x_{2}\right)$ is differentiable at $\left(x_{1}, x_{2}\right)$, the sum of the components of the hazard gradient vector can be equivalently represented by the derivative of the function of $-\ln \left[S_{X_{1}, X_{2}}\left(x_{1}, x_{2}\right)\right]$ along the direction of the vector $\vec{u}=(1,1)$. Thus, from Definition 5.2 .3 with $z=t$ and $\left(u_{1}, u_{2}\right)=(1,1)$ we have

$$
r\left(x_{1}, x_{2}\right)=\lim _{t \rightarrow 0} \frac{-\ln \left[S_{X_{1}, X_{2}}\left(x_{1}+t, x_{2}+t\right)\right]}{t}=-\frac{\partial}{\partial t} \ln \left[S_{X_{1}, X_{2}}\left(x_{1}+t, x_{2}+t\right)\right],
$$

evaluated at $t=0$. Substituting $S_{X_{1}, X_{2}}\left(x_{1}+t, x_{2}+t\right)$ by its alternative expression given by (7.4) we obtain

$$
r\left(x_{1}, x_{2}\right)=-\left.\frac{\partial}{\partial t} \ln \left[Q_{1}\left(x_{1}, t\right) Q_{2}\left(x_{2}, t\right) S_{X_{1}, X_{2}}(t, t) S_{X_{1}, X_{2}}\left(x_{1}, x_{2}\right)\right]\right|_{t=0} .
$$

If all the derivatives exist,

$$
r\left(x_{1}, x_{2}\right)=\left.\left[-\frac{d}{d t} \ln \left[S_{X_{1}, X_{2}}(t, t)\right]-\frac{\partial}{\partial t} \ln \left[Q_{1}\left(x_{1}, t\right)\right]-\frac{\partial}{\partial t} \ln \left[Q_{2}\left(x_{2}, t\right)\right]\right]\right|_{t=0}
$$

The conclusion is that $r\left(x_{1}, x_{2}\right)$ can be decomposed into a sum of a constant and two continuous and integrable functions, one that only depends on $x_{1}$ and the other that only depends on $x_{2}$. Notice that representation (7.5) has the same form advocated by Lemma 7.2.8, valid for GMO models.

Therefore, based on relation (7.5) we propose the following definition for the extended class of bivariate distributions $\mathcal{L}(\mathbf{x} ; \mathbf{A})$.

Definition 7.3.1. The nonnegative continuous bivariate survival function $S_{X_{1}, X_{2}}\left(x_{1}, x_{2}\right)$ belongs to the class $\mathcal{L}(\mathbf{x} ; \mathbf{A})$, where $\mathbf{x}=\left(x_{1}, x_{2}\right)$ and $\mathbf{A}=\left(a_{0}, A_{1}\left(x_{1}\right), A_{2}\left(x_{2}\right)\right)$, if and only if the sum of the components of its hazard gradient vector can be represented as

$$
r\left(x_{1}, x_{2}\right)=r_{1}\left(x_{1}, x_{2}\right)+r_{2}\left(x_{1}, x_{2}\right)=a_{0}+A_{1}\left(x_{1}\right)+A_{2}\left(x_{2}\right),
$$

where $a_{0}>0$ and the continuous integrable functions $A_{i}\left(x_{i}\right)$ are such that $A_{i}(0)=0$ and $A_{i}\left(x_{i}\right)>$ 
$-a_{0}$ for all $x_{i}>0, i=1,2$.

Observe that relation (5.2), that characterizes the class $\mathcal{L}(\mathbf{x} ; \mathbf{a})$, can be obtained from expression (7.6) substituting $A_{i}\left(x_{i}\right)=a_{i} x_{i}, i=1,2$. Therefore, all members of the class $\mathcal{L}(\mathbf{x} ; \mathbf{a})$ belong to the extended class $\mathcal{L}(\mathbf{x} ; \mathbf{A})$, introduced by Definition 7.3.1.

Remark 7.3.2 (Sum of hazard gradient vector components rule). Our construction of survival functions relies on the performance of $S_{X_{1}, X_{2}}\left(x_{1}, x_{2}\right)$ along the direction of the vector $\vec{u}=(1,1)$ and, according with Remark 7.1.1, we allow the existence of a singular component with support on the set $\Omega$ given by (2.10). Therefore, we can obtain absolutely continuous distributions as well as distributions composed by absolutely continuous and singular parts. When there exists a singular part, the hazard gradient vector components are no longer defined for $\left(x_{1}, x_{2}\right) \in \Omega$, so one should assume that

$$
r(x, x)=\frac{d}{d x}\left[-\ln S_{X_{1}, X_{2}}(x, x)\right]
$$

see related Remark 5.2.2.

The following statement is a consequence of Definition 7.3.1 and will be applied in Theorem 7.3.6, where we establish the equivalence between the class $\mathcal{L}(\mathbf{x} ; \mathbf{A})$ and the class of distributions possessing S-BLMP.

Lemma 7.3.3. Relation (7.6) that defines the class $\mathcal{L}(\mathbf{x} ; \mathbf{A})$ is equivalent to the functional equation

$$
r\left(x_{1}+t, x_{2}+t\right)=r\left(x_{1}+t, t\right)+r\left(t, x_{2}+t\right)-r(t, t)
$$

for all $x_{1}, x_{2} \geq 0$ and $t \geq 0$.

Proof. Let (7.6) be valid. Making the corresponding substitutions we obtain (7.7). Now suppose (7.7) holds true. Let $t=0$ to obtain $r\left(x_{1}, x_{2}\right)=r\left(x_{1}, 0\right)+r\left(0, x_{2}\right)-r(0,0)$. Denoting $r(0,0)=a_{0}$, notice that $r\left(x_{1}, 0\right)=a_{0}+A_{1}\left(x_{1}\right)$ and $r\left(0, x_{2}\right)=a_{0}+A_{2}\left(x_{2}\right)$ for some functions $A_{i}\left(x_{i}\right)$ such that $A_{i}(0)=0$ and $A_{i}\left(x_{i}\right)>-a_{0}$ for $x_{i}>0, i=1,2$. Thus we obtain (7.6).

Substitute in (7.6) $\psi\left(x_{1}, x_{2}\right)=r\left(x_{1}, x_{2}\right)-a_{0}$ and let $A_{1}\left(x_{1}\right)=\psi\left(x_{1}, 0\right)$ and $A_{2}\left(x_{2}\right)=\psi\left(0, x_{2}\right)$. Hence we get the functional equation

$$
\psi\left(x_{1}, x_{2}\right)=\psi\left(x_{1}, 0\right)+\psi\left(0, x_{2}\right)
$$

with solution

$$
\psi\left(x_{1}, x_{2}\right)=A_{1}\left(x_{1}\right)+A_{2}\left(x_{2}\right) .
$$

Therefore, the class $\mathcal{L}(\mathbf{x} ; \mathbf{A})$ can be equivalently defined by (7.6) or by the functional equations (7.7) or (7.8).

In Theorem 7.3.4 we provide the general expression for the survival function of the members of the class $\mathcal{L}(\mathbf{x} ; \mathbf{A})$.

Theorem 7.3.4. If $S_{X_{1}, X_{2}}\left(x_{1}, x_{2}\right)$ is continuous with continuous first partial derivatives, with a possible exception on the set $\Omega$ given by (2.10), then the defining relation (7.6) is fulfilled if and only if the corresponding joint survival function can be represented by

$$
S_{X_{1}, X_{2}}\left(x_{1}, x_{2}\right)= \begin{cases}S_{X_{1}}\left(x_{1}-x_{2}\right) \exp \left\{-a_{0} x_{2}-I_{1}\left(x_{1}\right)+I_{1}\left(x_{1}-x_{2}\right)-I_{2}\left(x_{2}\right)\right\}, & \text { if } x_{1} \geq x_{2} \geq 0 \\ S_{X_{2}}\left(x_{2}-x_{1}\right) \exp \left\{-a_{0} x_{1}-I_{1}\left(x_{1}\right)-I_{2}\left(x_{2}\right)+I_{2}\left(x_{2}-x_{1}\right)\right\}, & \text { if } x_{2} \geq x_{1} \geq 0\end{cases}
$$


where $\frac{d}{d x} I_{i}(x)=A_{i}(x), i=1,2$.

Proof. Assume (7.6) is fulfilled. Following the same steps as in the proof of Theorem 5.2.11 in Section 5.2, we obtain

$$
S_{X_{1}, X_{2}}\left(x_{1}, x_{2}\right)= \begin{cases}S_{X_{1}}\left(x_{1}-x_{2}\right) \exp \left\{-\int_{0}^{x_{2}}\left[a_{0}+A_{1}\left(x_{1}-x_{2}+t\right)+A_{2}(t)\right] \mathrm{d} t\right\}, & \text { if } x_{1} \geq x_{2} \geq 0, \\ S_{X_{2}}\left(x_{2}-x_{1}\right) \exp \left\{-\int_{0}^{x_{1}}\left[a_{0}+A_{1}(t)+A_{2}\left(x_{2}-x_{1}+t\right)\right] \mathrm{d} t\right\}, & \text { if } x_{2} \geq x_{1} \geq 0 .\end{cases}
$$

Taking into account that $\frac{d}{d x} I_{i}\left(x_{i}\right)=A_{i}\left(x_{i}\right)$, we have $\int_{a}^{b} A_{i}(u) \mathrm{d} u=I_{i}(b)-I_{i}(a), i=1,2$. In particular, notice that $I_{1}(0)=I_{2}(0)=0$.

Let $x_{1} \geq x_{2} \geq 0$. To evaluate $\int_{0}^{x_{2}} A_{1}\left(x_{1}-x_{2}+t\right) \mathrm{d} t$, consider the change of variable $u=x_{1}-x_{2}+t$ and notice that $\mathrm{d} u=\mathrm{d} t$ to obtain

$$
\int_{0}^{x_{2}} A_{1}\left(x_{1}-x_{2}+t\right) \mathrm{d} t=\int_{x_{1}-x_{2}}^{x_{1}} A_{1}(u) \mathrm{d} u=I_{1}\left(x_{1}\right)-I_{1}\left(x_{1}-x_{2}\right) .
$$

Solving the integrals in the foregoing equation we get (7.9). We proceed similarly when $x_{2} \geq x_{1} \geq 0$ and the necessary part of the statement is proved.

Now suppose that the joint survival $S_{X_{1}, X_{2}}\left(x_{1}, x_{2}\right)$ function is given by (7.9). Direct calculus show that hazard gradient vector components $r_{i}\left(x_{1}, x_{2}\right)=-\frac{\partial}{\partial x_{i}} \ln S_{X_{1}, X_{2}}\left(x_{1}, x_{2}\right), i=1,2$, should satisfy relations

$$
r_{1}\left(x_{1}, x_{2}\right)= \begin{cases}r_{X_{1}}\left(x_{1}-x_{2}\right)+A_{1}\left(x_{1}\right)-A_{1}\left(x_{1}-x_{2}\right), & \text { if } x_{1}>x_{2} \geq 0 \\ -r_{X_{2}}\left(x_{2}-x_{1}\right)+a_{0}+A_{1}\left(x_{1}\right)+A_{2}\left(x_{2}-x_{1}\right), & \text { if } x_{2}>x_{1} \geq 0\end{cases}
$$

and

$$
r_{2}\left(x_{1}, x_{2}\right)= \begin{cases}-r_{X_{1}}\left(x_{1}-x_{2}\right)+a_{0}+A_{1}\left(x_{1}-x_{2}\right)+A_{2}\left(x_{2}\right), & \text { if } x_{1}>x_{2} \geq 0 \\ r_{X_{2}}\left(x_{2}-x_{1}\right)+A_{2}\left(x_{2}\right)-A_{2}\left(x_{2}-x_{1}\right), & \text { if } x_{2}>x_{1} \geq 0\end{cases}
$$

In particular, when $x_{1}=x_{2}=x \geq 0$, from (7.9) and Remark 7.3.2 we have

$$
r(x, x)=\frac{d}{d x}\left[-\ln S_{X_{1}, X_{2}}(x, x)\right]=\frac{d}{d x}\left[a_{0} x+I_{1}(x)+I_{2}(x)\right]=a_{0}+A_{1}(x)+A_{2}(x),
$$

and condition (7.6) is fulfilled.

Remark 7.3.5 (Bounds for marginal failure rates). Since $r_{i}\left(x_{1}, x_{2}\right) \geq 0, i=1,2$, from (7.10), let $x_{1}=0$ to obtain $0 \leq r_{X_{2}}(x) \leq a_{0}+A_{2}(x)$. Analogously, from (7.11) we obtain $0 \leq r_{X_{1}}(x) \leq$ $a_{0}+A_{1}(x)$. Therefore, the behaviour of the marginal failure rate functions $r_{X_{i}}(x)$ is limited from above by a constant $a_{0}$ added to the corresponding function $A_{i}(x), i=1,2$. See also related Remark 5.3.3. These restrictions are important and have to be taken into account when generating members of the class $\mathcal{L}(\mathbf{x} ; \mathbf{A})$, see Section 7.4.

\subsubsection{Equivalence between the class $\mathcal{L}(\mathrm{x} ; \mathrm{A})$ and distributions possessing $\mathrm{S}-$ BLMP}

Following the same arguments as in Theorems 5.2.11 and 6.2.9, where we established the class $\mathcal{L}(\mathbf{x} ; \mathbf{a})$ can be characterized by LS-BLMP, in Theorem 7.3.6 we show that S-BLMP characterizes 
the distributions belonging to the class $\mathcal{L}(\mathbf{x} ; \mathbf{A})$. Having this equivalence at hand, we prove the characterization Theorem 7.3.8 which is, in fact, an additional bridge between the class $\mathcal{L}(\mathbf{x} ; \mathbf{A})$ and the distributions possessing S-BLMP.

The next theorem establishes the equivalence between the class of distributions possessing SBLMP and the class $\mathcal{L}(\mathbf{x} ; \mathbf{A})$.

Theorem 7.3.6. The S-BLMP given by relation (6.5) characterizes the distributions belonging to the class $\mathcal{L}(\mathbf{x} ; \mathbf{A})$ defined by (7.6).

Proof. Suppose $S_{X_{1}, X_{2}}\left(x_{1}, x_{2}\right)$ possesses S-BLMP, so that (6.8) is satisfied, for it is equivalent to (6.5), see Section 6.2. Let $x_{1} \geq x_{2} \geq 0$ to obtain

$$
S_{X_{1}, X_{2}}\left(x_{1}+t, x_{2}+t\right)=S_{X_{1}}\left(x_{1}-x_{2}\right) \exp \left\{\int_{0}^{x_{2}+t} r\left(x_{1}-x_{2}+u, u\right) \mathrm{d} u\right\} .
$$

In a similar way one may obtain corresponding expressions for

$$
S_{X_{1}, X_{2}}\left(x_{1}+t, t\right), S_{X_{1}, X_{2}}\left(t, x_{2}+t\right), S_{X_{1}, X_{2}}\left(x_{1}, x_{2}\right) \quad \text { and } \quad S_{X_{1}, X_{2}}(t, t)
$$

In particular, when $x_{1}=x_{2}=t \geq 0$, we assume that $\frac{d}{d t}\left[-\ln S_{X_{1}, X_{2}}(t, t)\right]=r(t, t)$, see Remark 7.3.2. Substituting these quantities in (6.8), after some algebra (taking logarithm in both sides and differentiating with respect to $t$ ) we obtain the functional equation (7.7), which is valid for $x_{1} \geq x_{2} \geq 0$ and all $t \geq 0$. From Lemma 7.3.3, (7.7) is equivalent to the defining relation (7.6), which characterizes the class $\mathcal{L}(\mathbf{x} ; \mathbf{A})$. The same is the conclusion when $x_{2} \geq x_{1} \geq 0$. Therefore, $S_{X_{1}, X_{2}}\left(x_{1}, x_{2}\right)$ belongs to the class $\mathcal{L}(\mathbf{x} ; \mathbf{A})$.

Now suppose $S_{X_{1}, X_{2}}\left(x_{1}, x_{2}\right)$ belongs to the class $\mathcal{L}(\mathbf{x} ; \mathbf{A})$ defined by relation (7.6). From Theorem 7.3.4, $S_{X_{1}, X_{2}}\left(x_{1}, x_{2}\right)$ can be represented by the general expression (7.9) whenever $S_{X_{1}, X_{2}}\left(x_{1}, x_{2}\right)$ is continuous with continuous first partial derivatives. Thus, from (7.9), it is direct to check that (6.8) holds true. But, as already noted, relation (6.8) is equivalent to (6.5). According with Definition 7.2.1, the survival function possesses the S-BLMP, finishing the proof.

From Theorems 7.3.4 and 7.3.6 we arrive to the following

Theorem 7.3.7. Let $S_{X_{1}, X_{2}}\left(x_{1}, x_{2}\right)$ be continuous with continuous first partial derivatives, possibly non existing on the set $\Omega$ given by (2.10). Then bivariate survival functions exhibiting S-BLMP given by (6.5) can be alternatively characterized by the general expression (7.9).

In the next theorem we obtain a functional equation relating the survival functions of $\left(X_{1}, X_{2}\right)$ and of its corresponding residual lifetime vector $\mathbf{X}_{t}$. This functional equation also characterizes the members of the class $\mathcal{L}(\mathbf{x} ; \mathbf{A})$.

Theorem 7.3.8. The joint survival function of $\left(X_{1}, X_{2}\right)$ is given by expression (7.9) if and only if holds true the functional equation

$$
S_{X_{1}, X_{2}}\left(x_{1}+t, x_{2}+t\right)=S_{X_{1}, X_{2}}\left(x_{1}, x_{2}\right) S_{X_{1}, X_{2}}(t, t) Q_{1}\left(x_{1}, t\right) Q_{2}\left(x_{2}, t\right),
$$

where the functions

$$
Q_{i}\left(x_{i}, t\right)=\exp \left\{-\left[I_{i}\left(x_{i}+t\right)-I_{i}\left(x_{i}\right)-I_{i}(t)\right]\right\},
$$

with $\frac{d}{d x} I_{i}(x)=A_{i}(x), i=1,2$. 
Proof. Suppose $x_{1} \geq x_{2} \geq 0$. From (7.9) we have

$$
S_{X_{1}, X_{2}}(t, t)=\exp \left\{-a_{0} t-I_{1}(t)-I_{2}(t)\right\} .
$$

In addition, from (7.9) the expression for $S_{X_{1}, X_{2}}\left(x_{1}+t, x_{2}+t\right)$ is

$$
\begin{aligned}
S_{X_{1}, X_{2}}\left(x_{1}+t, x_{2}+t\right) & =S_{X_{1}}\left(x_{1}-x_{2}\right) \\
& \times \exp \left\{-a_{0}\left(x_{2}+t\right)-I_{1}\left(x_{1}+t\right)+I_{1}\left(x_{1}-x_{2}\right)-I_{2}\left(x_{2}+t\right)\right\},
\end{aligned}
$$

which is identical with (7.12). The result is the same if $x_{2} \geq x_{1} \geq 0$ and the first part of the theorem is proved.

Conversely, suppose relation (7.12) holds true. Define the continuous function $S\left(x_{1}, x_{2}\right)$ such that

$$
S_{X_{1}, X_{2}}\left(x_{1}, x_{2}\right)=S\left(x_{1}, x_{2}\right) \exp \left\{-I_{1}\left(x_{1}\right)-I_{2}\left(x_{2}\right)\right\} .
$$

Notice that $S\left(x_{1}, x_{2}\right)$ do not need to be a bivariate survival function. Substituting (7.14) in (7.12) we obtain the functional equation

$$
S\left(x_{1}+t, x_{2}+t\right)=S\left(x_{1}, x_{2}\right) S(t, t),
$$

which is valid for all nonnegative $x_{1}, x_{2}$ and $t$. Consider $x_{1} \geq x_{2} \geq 0$ to obtain the only continuous solution of the previous functional equation as

$$
S\left(x_{1}, x_{2}\right)=S\left(x_{1}-x_{2}, 0\right) \exp \left[-a_{0} x_{2}\right]
$$

for some $a_{0}>0$. From (7.14) we have

$$
\begin{aligned}
S\left(x_{1}-x_{2}, 0\right) & =S_{X_{1}, X_{2}}\left(x_{1}-x_{2}, 0\right) \exp \left[I_{1}\left(x_{1}-x_{2}\right)\right] \\
& =S_{X_{1}}\left(x_{1}-x_{2}\right) \exp \left[I_{1}\left(x_{1}-x_{2}\right)\right],
\end{aligned}
$$

since $I_{2}(0)=0$, see the proof of Theorem 7.3.4. Therefore

$$
S_{X_{1}, X_{2}}\left(x_{1}, x_{2}\right) \exp \left[I_{1}\left(x_{1}\right)+I_{2}\left(x_{2}\right)\right]=S_{X_{1}, X_{2}}\left(x_{1}-x_{2}, 0\right) \exp \left[I_{1}\left(x_{1}-x_{2}\right)\right] \exp \left[-a_{0} x_{2}\right],
$$

i.e. $S_{X_{1}, X_{2}}\left(x_{1}, x_{2}\right)$ is represented by (7.9). Identical steps and conclusion follow if $x_{2} \geq x_{1} \geq 0$, finishing the proof.

Corollary 7.3.9. Relation (7.12) characterizes the class $\mathcal{L}(\mathbf{x} ; \mathbf{A})$ as well as the class of bivariate distributions possessing $S$-BLMP.

Proof. The result is a direct consequence of Theorems 7.3.6 and 7.3.8.

Remark 7.3.10. Notice that Theorem 7.3.8 holds true without the assumption of continuity of the first partial derivatives of $S_{X_{1}, X_{2}}\left(x_{1}, x_{2}\right)$. But this condition is necessary to obtain the remaining equivalence relations, see Remark 7.1.1.

The equivalences so far obtained are summarized in Figure 7.1 in Section 7.7. 


\subsubsection{Stochastic representation}

We will obtain a stochastic representation of the class $\mathcal{L}(\mathbf{x} ; \mathbf{A})$ assuming additional properties for the functions $A_{i}\left(x_{i}\right), i=1,2$, given in Definition 7.3.1. In fact, we have the following result.

Theorem 7.3.11. Let the joint survival function of $\left(X_{1}, X_{2}\right)$ belong to the class $\mathcal{L}(\mathbf{x} ; \mathbf{A})$ and the continuous and integrable functions $A_{i}\left(x_{i}\right)$ in (7.6) be strictly increasing with $\lim _{x_{i} \rightarrow \infty} A_{i}\left(x_{i}\right)=\infty$, $i=1,2$. Then there exists a random vector $\left(Z_{1}, Z_{2}\right)$ independent of $\left(X_{1}, X_{2}\right)$ such that $\mathbf{X}_{t}=$ $\left(\min \left\{X_{1}, Z_{1}\right\}, \min \left\{X_{2}, Z_{2}\right\}\right)$.

Proof. From Theorem 7.3.4 it follows that if the joint survival function of $\left(X_{1}, X_{2}\right)$ belongs to the class $\mathcal{L}(\mathbf{x} ; \mathbf{A})$ and is continuous with continuous first partial derivatives, then $S_{X_{1}, X_{2}}\left(x_{1}, x_{2}\right)$ is given by (7.9). According with Theorem 7.3.8, relation (7.9) can be equivalently represented by (7.12). Fix $t=t_{0}>0$ and let us introduce the functions

$$
I_{i}\left(x_{i}+t_{0}\right)-I_{i}\left(x_{i}\right)-I_{i}\left(t_{0}\right), \quad i=1,2,
$$

entering in the exponent of (7.13). Since $A_{i}\left(x_{i}\right)=\frac{d}{d x_{i}} I_{i}\left(x_{i}\right)$ are increasing in $x_{i}, i=1,2$ we have

$$
\frac{d}{d x_{i}}\left[I_{i}\left(x_{i}+t_{0}\right)-I_{i}\left(x_{i}\right)-I_{i}\left(t_{0}\right)\right]=A_{i}\left(x_{i}+t_{0}\right)-A_{i}\left(x_{i}\right)>0, \quad i=1,2,
$$

i.e., the functions in (7.15) are increasing in $x_{i}$. Observe that when $x_{i}=0$ we have $I_{i}\left(t_{0}\right)-I_{i}(0)-$ $I_{i}\left(t_{0}\right)=0$. From the monotonicity of $A_{i}\left(x_{i}\right)$, it follows that $I_{i}\left(x_{i}+t_{0}\right)-I_{i}\left(x_{i}\right)=\int_{x_{i}}^{x_{i}+t_{0}} A_{i}(u) \mathrm{d} u \geq$ $t_{0} A_{i}\left(x_{i}\right)$. Thus, taking into account the conditions $\lim _{x_{i} \rightarrow \infty} A_{i}\left(x_{i}\right)=\infty, i=1,2$, we arrive to

$$
\lim _{x_{i} \rightarrow \infty}\left[I_{i}\left(x_{i}+t_{0}\right)-I_{i}\left(x_{i}\right)-I_{i}\left(t_{0}\right)\right]=\infty
$$

Therefore, the functions given in (7.15) share the properties of cumulative failure rate functions of continuous nonnegative random variables to be denoted by $Z_{i}, i=1,2$. The survival function of $Z_{i}$ depends on $t_{0}$ and is given by $Q_{i}\left(x_{i}, t_{0}\right)$ in $(7.13), i=1,2$. Thus, the product $Q_{1}\left(x_{1}, t_{0}\right) Q_{2}\left(x_{2}, t_{0}\right)$ is the joint survival function of the random vector $\left(Z_{1}, Z_{2}\right)$ with independent marginals. Since $\frac{S_{X_{1}, X_{2}}\left(x_{1}+t, x_{2}+t\right)}{S_{X_{1}, X_{2}}(t, t)}$ is the joint survival function of $\mathbf{X}_{t}$, the required stochastic representation follows from (7.12).

Remark 7.3.12. The class $\mathcal{L}(\mathbf{x} ; \mathbf{a})$ introduced in Chapter 5 is characterized by the relation $r\left(x_{1}, x_{2}\right)=$ $a_{0}+a_{1} x_{1}+a_{2} x_{2}$, so that we have $A_{i}\left(x_{i}\right)=a_{i} x_{i}, a_{i}, x_{i} \geq 0, i=1,2$. Therefore, the conditions stated in Theorem 7.3.11 are satisfied. Due to the linear form of the functions involved, the stochastic relation between $\left(X_{1}, X_{2}\right)$ and $\mathbf{X}_{t}$ stated in Theorem 7.3.11 admits a simple interpretation in terms of shocks, represented by the random variables $Z_{1}$ and $Z_{2}$, governed by independent Poisson processes.

Remark 7.3.13 (Reliability property). Notice that expression (7.15) besides increasing in $x_{i}$ is also increasing in $t_{0}$, meaning that $\frac{S_{X_{1}, X_{2}}\left(x_{1}+t_{1}, x_{2}+t_{1}\right)}{S_{X_{1}, X_{2}}\left(x_{1}, x_{2}\right)} \geq \frac{S_{X_{1}, X_{2}}\left(x_{1}+t_{2}, x_{2}+t_{2}\right)}{S_{X_{1}, X_{2}}\left(x_{1}, x_{2}\right)}$ for $t_{1} \geq t_{2}>0$. Therefore, whenever $A_{i}\left(x_{i}\right)$ are strictly increasing in $x_{i}, i=1,2, S_{X_{1}, X_{2}}\left(x_{1}, x_{2}\right)$ is bivariate increasing failure rate (BIFR), according with the Definition 6.3.2.

Example 7.3.14. Consider the GMO distributions given by (3.1). Let $H_{T_{i}}(x)=\lambda_{i} x^{3}, \lambda_{i}>0$, 
$i=1,2$, and $H_{T_{3}}(x)=a_{0} x, a_{0}>0$, to obtain

$$
S_{X_{1}, X_{2}}\left(x_{1}, x_{2}\right)=\exp \left\{-\lambda_{1} x_{1}^{3}-\lambda_{2} x_{2}^{3}-a_{0} \max \left(x_{1}, x_{2}\right)\right\} .
$$

The sum of the components of the hazard gradient vector is $r\left(x_{1}, x_{2}\right)=a_{0}+3 \lambda_{1} x_{1}^{2}+3 \lambda_{2} x_{2}^{2}$, so that $A_{i}\left(x_{i}\right)=3 \lambda_{i} x_{i}^{2}, i=1,2$ in defining relation (7.6). From Lemma 7.2.8 this distribution possesses $S$-BLMP and from Theorem 7.3.4, with $I_{i}\left(x_{i}\right)=\lambda_{i} x_{i}^{3}, i=1,2$, admits the representation

$$
S_{X_{1}, X_{2}}\left(x_{1}, x_{2}\right)= \begin{cases}\exp \left\{-\lambda_{1}\left(x_{1}-x_{2}\right)^{3}-a_{0}\left(x_{1}-x_{2}\right)\right\} \exp \left\{-a_{0} x_{2}-\lambda_{1} x_{1}^{3}+\lambda_{1}\left(x_{1}-x_{2}\right)^{3}-\lambda_{2} x_{2}^{3}\right\}, & \text { if } x_{1} \geq x_{2} \geq 0, \\ \exp \left\{-\lambda_{2}\left(x_{2}-x_{1}\right)^{3}-a_{0}\left(x_{2}-x_{1}\right)\right\} \exp \left\{-a_{0} x_{1}-\lambda_{1} x_{1}^{3}-\lambda_{2} x_{2}^{3}+\lambda_{2}\left(x_{2}-x_{1}\right)^{3}\right\}, & \text { if } x_{2} \geq x_{1} \geq 0 .\end{cases}
$$

Applying Theorem 7.3.8,

$$
\begin{aligned}
S_{X_{1}, X_{2}}\left(x_{1}+t, x_{2}+t\right)= & S_{X_{1}, X_{2}}\left(x_{1}, x_{2}\right) S_{X_{1}, X_{2}}(t, t) \\
& \times \exp \left\{-\left[\lambda_{1}\left(\left(x_{1}+t\right)^{3}-x_{1}^{3}-t^{3}\right)+\lambda_{2}\left(\left(x_{2}+t\right)^{3}-x_{2}^{3}-t^{3}\right)\right]\right\} .
\end{aligned}
$$

Since $A_{i}\left(x_{i}\right)=3 \lambda_{i} x_{i}^{2}$, then $A_{i}(0)=0$ and $A_{i}\left(x_{i}\right)$ is strictly increasing in $x_{i}$ and unbounded from above, $i=1$, 2. From Theorem 7.3.11, the residual lifetime vector admits the stochastic representation $\mathbf{X}_{t}=\left(\min \left\{X_{1}, Z_{1}\right\}, \min \left\{X_{2}, Z_{2}\right\}\right)$, where $Z_{1}$ and $Z_{2}$ are two absolutely continuous nonnegative independent random variables such that $S_{Z_{i}}\left(x_{i}, t\right)=\exp \left\{-3 \lambda_{i} t\left(x_{i}^{2}+t x_{i}\right)\right\}$, i.e., $Z_{i}$ follow distributions with parameters that depend on $t, i=1,2$.

\subsubsection{Characterization of absolutely continuous S-BLMP distributions}

The parameter $a_{0}$ plays an important role in the class $\mathcal{L}(\mathbf{x} ; \mathbf{a})$, being equivalent to LS-BLMP. In fact, if $a_{0}=f_{X_{1}}(0)+f_{X_{2}}(0)$ then the joint survival function $S_{X_{1}, X_{2}}\left(x_{1}, x_{2}\right)$ is absolutely continuous and, if $a_{0}<f_{X_{1}}(0)+f_{X_{2}}(0)$, the distribution exhibits a singular component with support on the set $\Omega$ given in (2.10), see Remark 5.2.18. Similar property can be established for distributions possessing $\mathrm{S}-\mathrm{BLMP}$, as is shown in the next theorem.

Theorem 7.3.15. Consider the members of the class $\mathcal{L}(\mathbf{x} ; \mathbf{A})$ with survival function given by (7.9) and let $X_{i}$ be absolutely continuous random variables with density function $f_{X_{i}}\left(x_{i}\right), i=1,2$. Then $a_{0} \leq f_{X_{1}}(0)+f_{X_{2}}(0)$. The survival function (7.9) is absolutely continuous if and only if $f_{X_{1}}(0)+$ $f_{X_{2}}(0)=a_{0}$.

Proof. Since distributions possessing S-BLMP property may have a singular component with support on the set $\Omega$ given by (2.10), let $\alpha=\mathbb{P}\left(X_{1}=X_{2}\right) \in[0,1]$. According with (2.11), let us decompose the continuous bivariate survival function $S_{X_{1}, X_{2}}\left(x_{1}, x_{2}\right)$ in (7.9) into an absolutely continuous part $S_{X_{1}, X_{2}}^{a c}\left(x_{1}, x_{2}\right)$ and a singular one $S_{X_{1}, X_{2}}^{s i}\left(x_{1}, x_{2}\right)$ so that

$$
S_{X_{1}, X_{2}}\left(x_{1}, x_{2}\right)=(1-\alpha) S_{X_{1}, X_{2}}^{a c}\left(x_{1}, x_{2}\right)+\alpha S_{X_{1}, X_{2}}^{s i}\left(\max \left\{x_{1}, x_{2}\right\}\right)
$$

Let us denote $(1-\alpha) f_{X_{1}, X_{2}}^{a c}\left(x_{1}, x_{2}\right)=\frac{\partial^{2}}{\partial x_{1} \partial x_{2}} S_{X_{1}, X_{2}}\left(x_{1}, x_{2}\right)$, see equation (5.13). To evaluate the parameter $\alpha$, notice that $1-\alpha=\mathbb{P}\left(X_{1} \neq X_{2}\right)=\mathbb{P}\left(X_{1}>X_{2}\right)+\mathbb{P}\left(X_{2}>X_{1}\right)$. We first compute

$$
\mathbb{P}\left(X_{1}>X_{2}\right)=\int_{0}^{\infty} \int_{0}^{u}(1-\alpha) f_{X_{1}, X_{2}}^{a c}(u, v) \mathrm{d} v \mathrm{~d} u .
$$


The inner integral $I(u)=\int_{0}^{u}(1-\alpha) f_{X_{1}, X_{2}}^{a c}(u, v) \mathrm{d} v$ gives

$$
\begin{aligned}
I(u)=f_{X_{1}}(u) & -f_{X_{1}}(0) \exp \left\{-a_{0} u-I_{1}(u)-I_{2}(u)\right\} \\
& -A_{1}(u) \exp \left\{-a_{0} u-I_{1}(u)-I_{2}(u)\right\} .
\end{aligned}
$$

Therefore,

$$
\begin{aligned}
\mathbb{P}\left(X_{1}>X_{2}\right) & =\int_{0}^{\infty} I(u) \mathrm{d} u \\
& =1-\int_{0}^{\infty}\left[\left(f_{X_{1}}(0)+A_{1}(u)\right) \exp \left\{-a_{0} u-I_{1}(u)-I_{2}(u)\right\}\right] \mathrm{d} u .
\end{aligned}
$$

Analogously, we obtain

$$
P\left(X_{2}>X_{1}\right)=1-\int_{0}^{\infty}\left[\left(f_{X_{2}}(0)+A_{2}(v)\right) \exp \left\{-a_{0} v-I_{1}(v)-I_{2}(v)\right\}\right] \mathrm{d} v .
$$

Thus,

$$
1-\alpha=2-\int_{0}^{\infty}\left[\left(f_{X_{1}}(0)+f_{X_{2}}(0)-a_{0}+a_{0}+A_{1}(y)+A_{2}(y)\right) \exp \left\{-a_{0} y-I_{1}(y)-I_{2}(y)\right\}\right] \mathrm{d} y .
$$

Notice that

$$
\int_{0}^{\infty}\left[\left(a_{0}+A_{1}(y)+A_{2}(y)\right) \exp \left\{-a_{0} y-I_{1}(y)-I_{2}(y)\right\}\right] \mathrm{d} y=1,
$$

being the integral of the density function of the random variable $X=\min \left(X_{1}, X_{2}\right)$ over its support. Hence

$$
\alpha=\int_{0}^{\infty}\left[\left(f_{X_{1}}(0)+f_{X_{2}}(0)-a_{0}\right) \exp \left\{-a_{0} y-I_{1}(y)-I_{2}(y)\right\}\right] \mathrm{d} y
$$

Since for all $y \geq 0$ we have $\exp \left\{-a_{0} y-I_{1}(y)-I_{2}(y)\right\} \geq 0$ and $\alpha=\mathbb{P}\left(X_{1}=X_{2}\right) \in[0,1]$, then $a_{0} \leq f_{X_{1}}(0)+f_{X_{2}}(0)$.

To conclude the proof, the survival function given by (7.9) is absolutely continuous if and only if $\alpha=0$, which is equivalent to the condition $f_{X_{1}}(0)+f_{X_{2}}(0)=a_{0}$.

Remark 7.3.16 (Lower and upper bounds for $a_{0}$ ). Additional conditions, which depend on the functional forms of $A_{i}\left(x_{i}\right), i=1,2$, and on the distributions of $X_{1}$ and $X_{2}$, have to be imposed so that $S_{X_{1}, X_{2}}^{a c}\left(x_{1}, x_{2}\right)$ and $S_{X_{1}, X_{2}}^{s i}\left(\max \left\{x_{1}, x_{2}\right\}\right)$ in Theorem 7.3 .15 are proper survival functions. As a result, improved lower and upper bounds for $a_{0}$ can be established. For example, in the case of $B L M P_{1}$, i.e., $A_{i}\left(x_{i}\right)=0, i=1,2$, see Theorem 3 in Kulkarni (2006) and for LS-BLMP, i.e., $A_{i}\left(x_{i}\right)=a_{i} x_{i}, a_{i} \geq 0, i=1,2$, see Theorem 5.2.14.

Example 7.3.17 (Singular component in GMO distributions). Consider the GMO distribution from Example 7.3.14 where $S_{X_{i}}\left(x_{i}\right)=\exp \left\{-\lambda_{i} x_{i}^{3}-a_{0} x_{i}\right\}, i=1,2$. We have $f_{X_{i}}(0)=a_{0}, i=1,2$, and $f_{X_{1}}(0)+f_{X_{2}}(0)=2 a_{0}>a_{0}$. According with Theorem 7.3.15, the corresponding survival function has an absolutely continuous and a singular component.

Example 7.3.18 (Gumbel's type bivariate distributions (5.3) and (5.23)). Consider the Gumbel's Type I bivariate exponential distribution given by (5.3). We have $S_{X_{i}}\left(x_{i}\right)=\exp \left\{-\lambda_{i} x_{i}\right\}$, i.e., $f_{X_{i}}(0)=\lambda_{i}, i=1,2$. From defining relation (7.6), $a_{0}=\lambda_{1}+\lambda_{2}$ and $A_{i}\left(x_{i}\right)=\theta \lambda_{1} \lambda_{2} x_{i}$, 
$i=1,2$. Thus, $f_{X_{1}}(0)+f_{X_{2}}(0)=a_{0}$ and the corresponding survival function is absolutely continuous. The last conclusion fails for the survival function given by (5.23) in Example 5.3.10, where $S_{X_{i}}\left(x_{i}\right)=\exp \left\{-\left(\lambda_{i}+\lambda_{3}\right) x_{i}\right\}$, and $f_{X_{i}}(0)=\lambda_{i}+\lambda_{3}, i=1,2$. From defining relation (7.6), $a_{0}=\lambda_{1}+\lambda_{2}+\lambda_{3}$. Thus, $f_{X_{1}}(0)+f_{X_{2}}(0)=a_{0}+\lambda_{3}>a_{0}$. Applying Theorem 7.3 .15 we conclude that the corresponding joint survival function has absolutely continuous and singular components.

\subsection{Generation of distributions possessing S-BLMP}

In this section we discuss the closure properties of the class $\mathcal{L}(\mathbf{x} ; \mathbf{A})$. In the sequel these properties, together with the methodology developed in Kulkarni (2006) to generate distributions possessing $\mathrm{BLMP}_{1}$, are used to construct distributions with S-BLMP.

\subsubsection{Closure properties}

Let $\mathcal{L}(\mathbf{x} ; \mathbf{D})$ denote the class of all survival functions possessing S-BLMP, according to Definition 7.2.1, with a parameter space $\Theta(\mathbf{D})$ established in Definition 7.3.1. Note that $\Theta(\mathbf{D})$ may have different decomposition for different vectors $\mathbf{D}=\left(D_{0}, D_{1}, D_{2}\right)$ satisfying (6.5) with $x_{1}, x_{2} \geq 0$.

The closure properties of the class $\mathcal{L}(\mathbf{x} ; \mathbf{D})$ are given in the following theorem.

Theorem 7.4.1. Denote by $\mathcal{L}(\mathbf{x} ; \mathbf{D})$ the class of survival functions possessing $S$-BLMP according to Definition 7.2.1. This class has the following closure properties.

(S1) If $\mathcal{S}_{1}, \mathcal{S}_{2} \in \mathcal{L}(\mathbf{x} ; \mathbf{D})$ then their product $\mathcal{S}_{1} \mathcal{S}_{2} \in \mathcal{L}(\mathbf{x} ; \mathbf{D})$;

(S2) If $\mathcal{S} \in \mathcal{L}(\mathbf{x} ; \mathbf{D})$ then $[\mathcal{S}]^{c} \in \mathcal{L}(\mathbf{x} ; \mathbf{D})$ for $c \geq 1$;

(S3) If $\mathcal{S}_{1}, \mathcal{S}_{2} \in \mathcal{L}(\mathbf{x} ; \mathbf{D})$ then $\left[\mathcal{S}_{1}\right]^{c_{1}}\left[\mathcal{S}_{2}\right]^{c_{2}} \in \mathcal{L}(\mathbf{x} ; \mathbf{D})$ for constants $c_{1}, c_{2} \geq 1$;

(S4) If $S_{Y_{1}, Y_{2}}\left(x_{1}, x_{2}\right)$ belongs to $\mathcal{L}(\mathbf{x} ; \mathbf{D})$ and $\alpha>0$, then $S_{X_{1}, X_{2}}\left(x_{1}, x_{2}\right)=S_{Y_{1}, Y_{2}}\left(\alpha x_{1}, \alpha x_{2}\right)$ also belongs to $\mathcal{L}(\mathbf{x} ; \mathbf{D})$;

(S5) If $S_{Y_{1}, Y_{2}}\left(x_{1}, x_{2}\right)$ belongs to $\mathcal{L}(\mathbf{x} ; \mathbf{D})$ and the exponentially distributed random variable $Y_{3}$ is independent of $\left(Y_{1}, Y_{2}\right)$, then $S_{X_{1}, X_{2}}\left(x_{1}, x_{2}\right)$ belongs to $\mathcal{L}(\mathbf{x} ; \mathbf{D})$, where $\left(X_{1}, X_{2}\right)=$ $\left(\min \left(Y_{1}, Y_{3}\right), \min \left(Y_{2}, Y_{3}\right)\right)$.

Proof. It is direct to check the distributions obtained in (S1) to (S5) satisfy relation (7.6).

Remark 7.4.2 (Extensions of the closure properties). Closure properties (S1) and (S3) can be easily extended to any finite number of survival functions belonging to $\mathcal{L}(\mathbf{x} ; \mathbf{D})$.

The next two examples illustrate applications of the closure properties (S1) and (S5). They will be useful to offer a general scheme to generate a wealth of distributions possessing S-BLMP.

Example 7.4.3 (An application of (S1) to stochastic representation (7.2)). Suppose $S_{Y_{1}, Y_{2}}\left(x_{1}, x_{2}\right)$ and $S_{Z_{1}, Z_{2}}\left(x_{1}, x_{2}\right)$ belong to the class $\mathcal{L}(\mathbf{x} ; \mathbf{A})$, where $\left(Y_{1}, Y_{2}\right)$ is assumed to be independent of $\left(Z_{1}, Z_{2}\right)$. In particular, let $S_{Z_{1}, Z_{2}}\left(x_{1}, x_{2}\right)$ be composed by independent nonnegative absolutely continuous marginals, so that the dependence function $H_{Z_{1}, Z_{2}}\left(x_{1}, x_{2}\right)=0$, for all $x_{1}, x_{2} \geq 0$. Apply the stochastic representation (7.2) and the closure property (S1) to obtain a new survival function $S_{X_{1}, X_{2}}\left(x_{1}, x_{2}\right)=S_{Y_{1}, Y_{2}}\left(x_{1}, x_{2}\right) S_{Z_{1}, Z_{2}}\left(x_{1}, x_{2}\right)$. The following three relations are fulfilled: 
- $r_{X_{i}}\left(x_{i}\right)=r_{Y_{i}}\left(x_{i}\right)+r_{Z_{i}}\left(x_{i}\right), i=1,2$;

- $r_{\mathbf{X}}\left(x_{1}, x_{2}\right)=r_{\mathbf{Y}}\left(x_{1}, x_{2}\right)+r_{\mathbf{Z}}\left(x_{1}, x_{2}\right)$, where $r_{\mathbf{X}}\left(x_{1}, x_{2}\right), r_{\mathbf{Y}}\left(x_{1}, x_{2}\right)$ and $r_{\mathbf{Z}}\left(x_{1}, x_{2}\right)$ denote the sum of the components of the hazard gradient vectors of $S_{X_{1}, X_{2}}\left(x_{1}, x_{2}\right), S_{Y_{1}, Y_{2}}\left(x_{1}, x_{2}\right)$ and $S_{Z_{1}, Z_{2}}\left(x_{1}, x_{2}\right)$, respectively;

- $H_{X_{1}, X_{2}}\left(x_{1}, x_{2}\right)=H_{Y_{1}, Y_{2}}\left(x_{1}, x_{2}\right)$.

Example 7.4.4 (Closure property (S5) applied to Generalized and Extended Marshall-Olkin distributions). Consider two independent nonnegative absolutely continuous random variables $Y_{1}$ and $Y_{2}$ such that their joint survival function belongs to the class $\mathcal{L}(\mathbf{x} ; \mathbf{A})$. Let $Y_{3}$ be independent of $\left(Y_{1}, Y_{2}\right)$ and exponentially distributed with parameter $a_{0}$. Consider the stochastic representation (2.9) and apply the closure property (S5) to obtain the corresponding GMO distribution that belongs to the class $\mathcal{L}(\mathbf{x} ; \mathbf{A})$, has survival function given by (3.1) and dependence function $H_{X_{1}, X_{2}}\left(x_{1}, x_{2}\right)=a_{0} \min \left(x_{1}, x_{2}\right)$, see Lemma 7.2.7.

Assume now that $Y_{1}$ and $Y_{2}$ are dependent random variables with joint survival function belonging to the class $\mathcal{L}(\mathbf{x} ; \mathbf{A})$. If $\left(Y_{1}, Y_{2}\right)$ is independent of $Y_{3}$, apply the stochastic representation (2.9) to obtain an EMO model with survival function given by (3.2) and dependence function $H_{X_{1}, X_{2}}\left(x_{1}, x_{2}\right)=H_{Y_{1}, Y_{2}}\left(x_{1}, x_{2}\right)+a_{0} \min \left(x_{1}, x_{2}\right)$. See equation (5.23) and Example 7.2.5 for particular cases. From closure property (S5) the resulting EMO model is a member of the class $\mathcal{L}(\mathbf{x} ; \mathbf{A})$. The following relations are valid:

- $r_{X_{i}}\left(x_{i}\right)=a_{0}+r_{Y_{i}}\left(x_{i}\right), i=1,2$;

- $r_{\mathbf{X}}\left(x_{1}, x_{2}\right)=a_{0}+r_{\mathbf{Y}}\left(x_{1}, x_{2}\right)$, where $r_{\mathbf{X}}\left(x_{1}, x_{2}\right)$ and $r_{\mathbf{Y}}\left(x_{1}, x_{2}\right)$ denote the sum of the components of the hazard gradient vectors of $S_{X_{1}, X_{2}}\left(x_{1}, x_{2}\right)$ and $S_{Y_{1}, Y_{2}}\left(x_{1}, x_{2}\right)$, respectively;;

- $S_{X_{1}, X_{2}}\left(x_{1}, x_{2}\right)$ and $S_{Y_{1}, Y_{2}}\left(x_{1}, x_{2}\right)$ share the same function $A_{i}\left(x_{i}\right), i=1,2$, in relation (7.6).

\subsubsection{Common building scheme}

In order to construct distributions possessing S-BLMP we take into account the characterizing Theorem 7.3.6, so that we equivalently consider distributions that belong to the class $\mathcal{L}(\mathbf{x} ; \mathbf{A})$.

For each pair of functions $A_{i}\left(x_{i}\right), i=1,2$, in relation (7.6), a specific building scheme that characterizes all members of the corresponding class $\mathcal{L}(\mathbf{x} ; \mathbf{A})$ should be used. For instance, the case $A_{i}\left(x_{i}\right)=0, i=1,2$, is considered in Theorem 3 in Kulkarni (2006), where the whole class of $\mathrm{BLMP}_{1}$ survival functions is obtained. If $A_{i}\left(x_{i}\right)=a_{i} x_{i}, a_{i} \geq 0, i=1,2$, we refer to Section 5.3 to obtain all possible survival functions having LS-BLMP.

Depending on the functional form of the functions $A_{i}\left(x_{i}\right), i=1,2$, the specific construction scheme may lead to complex relations for the parameter constraints of the resulting joint survival function given by expression (7.9) in Theorem 7.3.4.

Alternatively, we propose two common building schemes based on the closure properties of the class $\mathcal{L}(\mathbf{x} ; \mathbf{A})$ given in Theorem 7.4.1 and on the methodology for constructing $\mathrm{BLMP}_{1}$ distributions developed in Theorem 3 in Kulkarni (2006).

As mentioned in Remark 7.3.5, the marginal failure rates $r_{X_{i}}(x)$ are limited from above by $a_{0}+A_{i}(x), i=1,2$, so that one has to respect this restriction. Thus, we will consider the following two possible scenarios to generate S-BLMP distributions: 
(Scenario 1) The pre-specified continuous integrable functions $A_{i}\left(x_{i}\right)$ in Definition 7.3.1 of SBLMP are supposed to be nonnegative and to satisfy $\int_{0}^{\infty} A_{i}(u) \mathrm{d} u=\infty, i=1,2$;

(Scenario 2) The distributions possessing S-BLMP have pre-specified differentiable marginal failure rate functions $r_{X_{i}}\left(x_{i}\right), i=1,2$.

Considering Scenario 1, we propose the following three-step building procedure:

1. Start with a set of survival functions that possesses BLMP 1 , i.e., satisfy relation (7.6) with $A_{i}\left(x_{i}\right)=0$, for all $x_{i} \geq 0, i=1,2$. Denote this set of survival functions by $\mathcal{S}_{B L M P}^{1}$;

2. Use the closure property (S1) from Theorem 7.4.1 and multiply each member of $\mathcal{S}_{B L M P}^{1}$ by $S_{Y_{1}, Y_{2}}\left(x_{1}, x_{2}\right)=\exp \left\{-I_{1}\left(x_{1}\right)-I_{2}\left(x_{2}\right)\right\}$, where $I_{i}\left(x_{i}\right)=\int_{0}^{x_{i}} A_{i}(u) \mathrm{d} u, i=1,2$. The resulting survival functions will satisfy the defining relation (7.6) with the pre-specified functions $A_{i}\left(x_{i}\right)$, $i=1,2$, see Example 7.4.3;

3. Apply closure property (S5) from Theorem 7.4.1 to enrich the set of distributions obtained in the previous step.

Remark 7.4.5. Notice that in step 1 we only take into account the constant $a_{0}$ in relation (7.6), while in step 2 we introduce the two pre-specified continuous integrable functions $A_{i}\left(x_{i}\right), i=1,2$.

Remark 7.4.6. In the last step only the constant $a_{0}$ in relation (7.6) will be affected, see Example 7.4.4. Moreover, if the distribution obtained in step 2 is absolutely continuous, then, in step 3, the resulting survival function will display a singular component, with support on the set $\Omega$ given by (2.10).

Remark 7.4.7 (Limitation of the building scheme in Scenario 1). The building procedure suggested for Scenario 1 is a general one and unable to cover all the elements of the class $\mathcal{L}(\mathbf{x} ; \mathbf{A})$ for the given functions $A_{i}\left(x_{i}\right), i=1,2$. In fact, in Section 5.3 we obtained the Gumbel's Type I bivariate exponential distribution (5.3) using a specific procedure that characterizes nonnegative continuous survival functions such that $A_{i}\left(x_{i}\right)=a_{i} x_{i}$, with $a_{i} \geq 0, i=1,2$. This distribution is not an outcome of the three-step building scheme presented above. See also the related Remark 6.2.12.

Remark 7.4.8 (Obtaining different solutions). The obtained set of distributions is not unique, since it will depend on the choice of two failure rate functions in step 1. Considering other choices, at the end of step 3 different bivariate survival functions will be found, but sharing the same functions $A_{i}\left(x_{i}\right), i=1,2$, in relation (7.6), see Example 7.4 .9 in the sequel.

Example 7.4.9 (Building S-BLMP survival functions with given functions $\left.A_{i}\left(x_{i}\right), i=1,2\right)$. Let us obtain nonnegative continuous survival functions possessing $S$-BLMP such that $A_{1}\left(x_{1}\right)=\sqrt{x_{1}}$ and $A_{2}\left(x_{2}\right)=\ln \left(1+x_{2}\right)$ in defining relation (7.6). Notice that $A_{i}(0)=0, A_{i}\left(x_{i}\right)$ are nonnegative, $i=1,2$, with $I_{1}\left(x_{1}\right)=\int_{0}^{x_{1}} A_{1}(u) \mathrm{d} u=\frac{2 \sqrt{x_{1}^{3}}}{3}$ and $I_{2}\left(x_{2}\right)=\int_{0}^{x_{2}} A_{2}(u) \mathrm{d} u=\left(1+x_{2}\right) \ln \left(1+x_{2}\right)-x_{2}$, so that $I_{1}(x)$ and $I_{2}(x)$ are unbounded from above. Thus, the proposed building procedure can be applied. In step 1, choose two differentiable failure rate functions satisfying Theorem 3 in Kulkarni (2006) and obtain the corresponding BLMP 1 survival functions (for instance, consider the bivariate survival functions to be obtained in step 2 of Example 7.4.12). In step 2, consider $S_{Y_{1}, Y_{2}}\left(x_{1}, x_{2}\right)=$ $\exp \left\{-\frac{2 \sqrt{x_{1}^{3}}}{3}-\left(1+x_{2}\right) \ln \left(1+x_{2}\right)+x_{2}\right\}$. Finally, in step 3, use an exponentially distributed random 
variable $Y_{3}$ and apply the closure property (S5) from Theorem 7.4.1 to get the output. Notice that choosing two other failure rate functions in step 1 will lead to a different set of survival functions at the end of step 3.

In Scenario 2 one wishes to obtain survival functions possessing S-BLMP with prescribed differentiable marginal failure rates $r_{X_{i}}\left(x_{i}\right), i=1,2$. The building algorithm is as follows:

1. Choose a real constant $K>0$ and two differentiable failure rate functions $r_{Z_{i}}(x), i=1,2$, such that

(a) $0 \leq r_{Z_{i}}(x) \leq K$, for all $x \geq 0$;

(b) $r_{Z_{i}}(x)\left[K-r_{Z_{i}}(x)\right]+\frac{d}{d x} r_{Z_{i}}(x) \geq 0, \forall x \geq 0$;

(c) $r_{Z_{1}}(0)+r_{Z_{2}}(0)>K$;

(d) $\int_{0}^{x_{i}}\left[r_{X_{i}}(u)-r_{Z_{i}}(u)\right] \mathrm{d} u$ is non-decreasing and unbounded from above;

2. Apply the procedure presented in Theorem 3 in Kulkarni (2006) to obtain all BLMP survival $_{1}$ functions having as marginal failure rates the chosen functions $r_{Z_{i}}\left(x_{i}\right), i=1,2$, from step 1 . Denote this set of survival functions by $\mathcal{S}_{B L M P}^{2}$;

3. Define the nonnegative functions $\alpha_{i}\left(x_{i}\right)=r_{X_{i}}\left(x_{i}\right)-r_{Z_{i}}\left(x_{i}\right)$ and denote by $I_{i}\left(x_{i}\right)=\int_{0}^{x_{i}} \alpha_{i}(u) \mathrm{d} u$, $i=1,2$. To obtain the desired survival functions, according with the closure property (S1) in Theorem 7.4.1, multiply each member of $\mathcal{S}_{B L M P}^{2}$ by $S_{Y_{1}, Y_{2}}\left(x_{1}, x_{2}\right)=\exp \left\{-I_{1}\left(x_{1}\right)-I_{2}\left(x_{2}\right)\right\}$.

Remark 7.4.10. Notice that the restriction (a)-(c) on the marginal failure rate functions in step 1 are, in fact, the conditions in Theorem 3 in Kulkarni (2006). Moreover, we require condition (d) in order to ensure valid marginal cumulative failure rate functions $I_{i}\left(x_{i}\right), i=1,2$.

Remark 7.4.11 (Various possible solutions). The resulting set of survival functions under Scenario 2 is not unique, since it depends on the choice of the constant $K$ and on the two failure rate functions $r_{Z_{i}}(x), i=1,2$. Considering other choices, different distributions, with different functions $A_{i}\left(x_{i}\right)$, $i=1,2$, in relation (7.6) will result. Nonetheless, all bivariate survival functions obtained will share the same pre-specified marginal failure rates $r_{X_{i}}\left(x_{i}\right), i=1,2$.

In this second building procedure we did not make use of closure property (S5) from Theorem 7.4.1 as a final step since this property does not preserve the marginal failure rates, see Example 7.4.4.

Example 7.4.12 (Building S-BLMP survival functions from pre-specified differentiable marginal failure rates $\left.r_{X_{i}}\left(x_{i}\right), i=1,2\right)$. Let us obtain nonnegative continuous bivariate survival functions possessing S-BLMP with marginal failure rate functions given by $r_{X_{1}}\left(x_{1}\right)=\lambda_{1}-\frac{\lambda_{2}}{a+x_{1}^{2}}+a_{1} x_{1}^{3}$ and $r_{X_{2}}\left(x_{2}\right)=\lambda_{1}-\frac{\lambda_{2}}{a+x_{2}^{2}}+a_{2} x_{2}^{5}$, where $\lambda_{1}, \lambda_{2}, a>0$ and $\lambda_{1} \geq \frac{2 \lambda_{2}}{a}$. Notice that $\lambda_{1}-\frac{\lambda_{2}}{a+x^{2}} \leq \lambda_{1} \forall x \geq 0$. In step 1 let us choose $K=\lambda_{1}$, and consider $r_{Z_{1}}(x)=r_{Z_{2}}(x)=\lambda_{1}-\frac{\lambda_{2}}{a+x^{2}}$. Notice that with these choices, conditions (a)-(d) are satisfied. In step 2 we follow Section 5.4 in Kulkarni (2006) and obtain the set of survival functions $\mathcal{S}_{B L M P}^{2}$ given by

$$
S_{Z_{1}, Z_{2}}\left(x_{1}, x_{2}\right)= \begin{cases}\exp \left\{-\lambda_{1} x_{1}-\left(d-\lambda_{1}\right) x_{2}+\frac{\lambda_{2}}{\sqrt{a}} \arctan \left(\frac{x_{1}-x_{2}}{\sqrt{a}}\right)\right\}, & \text { if } x_{1} \geq x_{2} \geq 0, \\ \exp \left\{-\lambda_{1} x_{2}-\left(d-\lambda_{1}\right) x_{1}+\frac{\lambda_{2}}{\sqrt{a}} \arctan \left(\frac{x_{2}-x_{1}}{\sqrt{a}}\right)\right\}, & \text { if } x_{2} \geq x_{1} \geq 0,\end{cases}
$$


where the constant $d \in\left[\lambda_{1}, 2\left(\lambda_{1}-\frac{\lambda_{2}}{a}\right)\right]$. In the last step we consider $\alpha_{1}\left(x_{1}\right)=a_{1} x_{1}^{3}$ and $\alpha_{2}\left(x_{2}\right)=$ $a_{2} x_{2}^{5}$ to obtain the increasing and unbounded functions $I_{1}\left(x_{1}\right)=\frac{a_{1}}{4} x_{1}^{4}$ and $I_{2}\left(x_{2}\right)=\frac{a_{2}}{6} x_{2}^{6}$. Let $S_{Y_{1}, Y_{2}}\left(x_{1}, x_{2}\right)=\exp \left\{-\frac{a_{1}}{4} x_{1}^{4}-\frac{a_{2}}{6} x_{2}^{6}\right\}$ to obtain the desired survival functions as $S_{X_{1}, X_{2}}\left(x_{1}, x_{2}\right)=$ $S_{Z_{1}, Z_{2}}\left(x_{1}, x_{2}\right) S_{Y_{1}, Y_{2}}\left(x_{1}, x_{2}\right)$. Notice that the resulting distributions are absolutely continuous if $c=$ $2\left(\lambda_{1}-\frac{\lambda_{2}}{a}\right)$, otherwise have a singular component with support on the set $\Omega$ given by (2.10). Finally, choosing another value for $K$ and two other failure rate functions $r_{Z_{i}}\left(x_{i}\right), i=1,2$, in step 1 , a different set of survival functions will result at the end of step 3.

\subsection{Bivariate survival functions with non-aging survival copulas}

In this section, instead of memoryless dependence functions $H_{X_{1}, X_{2}}\left(x_{1}, x_{2}\right)$, we consider nonaging survival copulas $\bar{C}(u, v)$. Let us denote by $\mathcal{A}$ the class of continuous bivariate survival functions $S_{X_{1}, X_{2}}\left(x_{1}, x_{2}\right)$ such that $\left(X_{1}, X_{2}\right)$ and the corresponding residual lifetime vector $\mathbf{X}_{t}$ have the same survival copula for all $x_{1}, x_{2}$ and $t \geq 0$, i.e., $\bar{C}(u, v)=\bar{C}_{t}(u, v)$ for all $u, v \in[0,1]$. Therefore, the following functional equation

$$
\frac{\bar{C}\left(S_{X_{1}}\left(x_{1}+t\right), S_{X_{2}}\left(x_{2}+t\right)\right)}{\bar{C}\left(S_{X_{1}}(t), S_{X_{2}}(t)\right)}=\bar{C}\left(\frac{\bar{C}\left(S_{X_{1}}\left(x_{1}+t\right), S_{X_{2}}(t)\right)}{\bar{C}\left(S_{X_{1}}(t), S_{X_{2}}(t)\right)}, \frac{\bar{C}\left(S_{X_{1}}(t), S_{X_{2}}\left(x_{2}+t\right)\right)}{\bar{C}\left(S_{X_{1}}(t), S_{X_{2}}(t)\right)}\right)
$$

has to be satisfied for all $x_{1}, x_{2} \geq 0$ and $t \geq 0$. We will assume that the survival copula $\bar{C}$ is time invariant (or non-aging) if it corresponds to a member of the class $\mathcal{A}$.

All bivariate survival functions possessing $\operatorname{BLMP}_{1}$ belong to $\mathcal{A}$. It happens that this time invariance property is not restricted to $\mathrm{BLMP}_{1}$ survival functions. Other members of the class $\mathcal{A}$ are presented in Examples 7.5.1 and 7.5.2 in this section.

Let $\mathcal{D}(t)=\left\{(u, v) \in(0,1] \mid u=S_{X_{1}}(t), v=S_{X_{2}}(t), t>0\right\}$ represent the curve on the unit square parameterized by $t>0$. In such a case, from (7.17) we may obtain non-aging survival copula whenever $\bar{C}$ is invariant along the curve $\mathcal{D}(t)$. In particular, if the equality in distribution $X_{1} \stackrel{d}{=} X_{2}$ holds true, we have invariance of the survival copula along the main diagonal of the unit square.

Example 7.5.1 (Invariance along the main diagonal). The Cuadras-Augé copula

$$
\bar{C}_{\alpha}(u, v)=[\min (u v)]^{\alpha}[u v]^{1-\alpha}, \quad \alpha \in[0,1]
$$

is invariant on the main diagonal of the unit square, see Charpentier and Juri (2006). Let us initially consider equally distributed marginals $S_{X_{1}}(x)=S_{X_{2}}(x)=S_{X}(x)$. If $S_{X}(x)$ is exponentially distributed, then $S_{X_{1}, X_{2}}\left(x_{1}, x_{2}\right)=\bar{C}_{\alpha}\left(S_{X_{1}}\left(x_{1}\right), S_{X_{2}}\left(x_{2}\right)\right)$ is a particular case of Marshall-Olkin's bivariate exponential distribution, see Marshall and Olkin (1967), possessing BLMP 1 and, consequently, belonging to the class $\mathcal{A}$. Now fix a gamma distribution for $S_{X}(x)$. In this case, $B L M P_{1}$ does not hold true but the corresponding joint survival function still belongs to $\mathcal{A}$. In a third scenario, where $X_{1}$ and $X_{2}$ do not share the same distribution but are joined by the Cuadras-Augé survival copula, $S_{X_{1}, X_{2}}\left(x_{1}, x_{2}\right)$ neither possesses BLMP nor belongs to $\mathcal{A}$.

Example 7.5.2 (Invariance along a curve). The Marshall-Olkin survival copula

$$
\bar{C}_{\eta_{1}, \eta_{2}}(u, v)=\min \left(u^{1-\eta_{1}} v, u v^{1-\eta_{2}}\right), \quad \eta_{1}, \eta_{2} \in(0,1)
$$


is invariant on the curve $\left\{(u, v)=\left(z^{\eta_{1}}, z^{\eta_{2}}\right), z \in(0,1)\right\}$, see Charpentier and Juri (2006). Notice that when $\eta_{1}=\eta_{2}$ we obtain the Cuadras-Augé copula from Example 7.5.1. Initially let us consider a baseline survival function $S_{X}(x) x \geq 0$, and marginals $S_{X_{1}}(x)=\left[S_{X}(x)\right]^{\eta_{1}}$ and $S_{X_{2}}(x)=\left[S_{X}(x)\right]^{\eta_{2}}$. Then the corresponding joint survival function $S\left(x_{1}, x_{2}\right)=\bar{C}_{\eta_{1}, \eta_{2}}\left(S_{X_{1}}\left(x_{1}\right), S_{X_{2}}\left(x_{2}\right)\right)$ belongs to $\mathcal{A}$. In particular, if the marginals are exponentially distributed, not necessarily sharing the same parameter, then $S\left(x_{1}, x_{2}\right)$ possesses $B L M P_{1}$. But choosing $X_{1}$ exponentially distributed and $X_{2}$ beta distributed, say, the corresponding joint survival function is not a member of the class $\mathcal{A}$.

The cases considered in the last two examples depend on the choice of the marginal survival functions. A general invariance property can be obtained when we consider the Clayton survival copula. In such a case, for any marginals we have time invariant survival copulas, see Section 7.6 for a related discussion. We refer the reader to Section 4 in Charpentier and Juri (2006) for more details on time invariant copulas.

Remark 7.5.3. In Mulero and Pellerey (2010) it is given a characterization of the survival functions which simultaneously have Clayton copula and possess $B L M P_{1}$, see their Theorem 3.2.

In the next statement we establish a necessary condition to an absolutely continuous bivariate survival function be a member of the class $\mathcal{A}$.

Theorem 7.5.4. Let $S_{X_{1}, X_{2}}\left(x_{1}, x_{2}\right)$ be an absolutely continuous survival function belonging to the class $\mathcal{A}$. Then its survival copula satisfies the functional equation

$$
\bar{C}(u, v)=\left[u-\frac{f_{X_{2}}(0) \bar{C}^{2}(u, 1)}{a_{0}}\right] \bar{C}^{1}(u, v)+\left[v-\frac{f_{X_{1}}(0) \bar{C}^{1}(1, v)}{a_{0}}\right] \bar{C}^{2}(u, v),
$$

for all $u, v \in[0,1]$ and $a_{0}>0$, where $\bar{C}^{1}$ and $\bar{C}^{2}$ mean the partial derivative of $\bar{C}$ with respect to the first and second argument, correspondingly.

Proof. Take the derivative in (7.17) with respect to $t$ and substitute $t=0$ to get (7.18).

The knowledge of first partial derivatives of survival copula $\bar{C}(u, v)$ is sufficient to recover the distribution of $\min (U, V)$, where $U$ and $V$ are uniformly distributed with survival copula $\bar{C}(u, v)$. In fact, $\mathbb{P}(\min (U, V)>t)=\bar{C}(t, t)$ for $t \in[0,1]$. Substitute $u=v=t$ in (7.18) to get the corresponding equation (and the main diagonal copula).

In the next remark we show two known functional equations which are particular cases of the necessary condition given by relation (7.18).

Remark 7.5.5. Additionally to the assumptions of Theorem 7.5.4, let $f_{X_{1}}(0)=f_{X_{2}}(0)$ in relation (7.18). Then

$$
\bar{C}(u, v)=\left[u-\frac{\bar{C}^{2}(u, 1)}{2}\right] \bar{C}^{1}(u, v)+\left[v-\frac{\bar{C}^{1}(1, v)}{2}\right] \bar{C}^{2}(u, v) .
$$

The same equation is obtained in Proposition 3 (ii) in Charpentier (2003) under the condition that $X_{1}$ and $X_{2}$ are uniformly distributed on the unit square, i.e. $f_{X_{1}}(0)=f_{X_{2}}(0)=1$.

Further, assume that $\bar{C}(u, v)$ is exchangeable. Thus, $\bar{C}^{2}(u, 1)=\bar{C}^{1}(1, u), \bar{C}^{2}(u, v)=\bar{C}^{1}(v, u)$ and the last equation transforms into

$$
\bar{C}(u, v)=\left[u-\frac{\bar{C}^{1}(1, u)}{2}\right] \bar{C}^{1}(u, v)+\left[v-\frac{\bar{C}^{1}(1, v)}{2}\right] \bar{C}^{1}(v, u),
$$


see Proposition 3 on page 18 in Gourieroux and Monfort (2003).

\subsection{A stronger version of S-BLMP}

As we noticed in Section 2.3, in one of the first attempts to extend the lack of memory property to the bivariate dimension Marshall and Olkin (1967) considered the functional equation

$$
S_{X_{1}, X_{2}}\left(x_{1}+t_{1}, x_{2}+t_{2}\right)=S_{X_{1}, X_{2}}\left(x_{1}, x_{2}\right) S_{X_{1}, X_{2}}\left(t_{1}, t_{2}\right),
$$

for all $x_{1}, x_{2}, t_{1}, t_{2}>0$, that expresses the residual life be independent of age. According with Theorem 2.1 in Marshall and Olkin (1967), the only continuous solution of this functional equation is

$$
S_{X_{1}, X_{2}}\left(x_{1}, x_{2}\right)=\exp \left\{-b_{1} x_{1}-b_{2} x_{2}\right\}, b_{1}, b_{2}>0 .
$$

Therefore, in a similar way, it is natural to investigate when the dependence function of the random vector $\mathbf{X}_{\left(t_{1}, t_{2}\right)}=\left(X_{1}-t_{1}, X_{2}-t_{2} \mid X_{1}>t_{1}, X_{2}>t_{2}\right)$ is the same as that of $\mathbf{X}=\left(X_{1}, X_{2}\right)$, i.e., when

$$
H_{\mathbf{X}}\left(x_{1}, x_{2}\right)=H_{\mathbf{X}_{\left(t_{1}, t_{2}\right)}}\left(x_{1}, x_{2}\right),
$$

for all $x_{1}, x_{2}, t_{1}, t_{2} \geq 0$ and to expect that the result in Theorem 2.1 in Marshall and Olkin (1967) belongs to the set of survival functions to be obtained under condition (7.19). In fact, in Example 7.6.3 we show this last fact.

Let us consider the definition of dependence function given by equation (3.4) and apply the exponential representation (3.3) to relation (7.19) and get the equivalent functional equation

$$
\begin{aligned}
H_{X_{1}, X_{2}}\left(x_{1}+t_{1}, x_{2}+t_{2}\right) & =H_{X_{1}, X_{2}}\left(x_{1}+t_{1}, t_{2}\right)+H_{X_{1}, X_{2}}\left(t_{1}, x_{2}+t_{2}\right) \\
& +H_{X_{1}, X_{2}}\left(x_{1}, x_{2}\right)-H_{X_{1}, X_{2}}\left(t_{1}, t_{2}\right)
\end{aligned}
$$

in terms of dependence function $H_{X_{1}, X_{2}}\left(x_{1}, x_{2}\right)$, which has to be satisfied for all $x_{1}, x_{2}, t_{1}, t_{2} \geq 0$.

Remark 7.6.1. Notice that in (7.20), the boundary conditions for a dependence function of two nonnegative random variables $X_{1}$ and $X_{2}$, namely $H_{X_{1}, X_{2}}\left(0, x_{2}\right)=H_{X_{1}, X_{2}}\left(x_{1}, 0\right)=0$ for all $x_{1}, x_{2} \geq 0$, always hold true.

In the next theorem we characterize all continuous solution of the functional equation (7.20).

Theorem 7.6.2. The only continuous solution of the functional equation (7.20) is $H_{X_{1}, X_{2}}\left(x_{1}, x_{2}\right)=$ $c x_{1} x_{2}$ for some constant $c$ and all $x_{1}, x_{2} \geq 0$.

Proof. Let $t_{2}=0$ in the functional equation (7.20) to obtain

$$
H_{X_{1}, X_{2}}\left(x_{1}+t_{1}, x_{2}\right)=H_{X_{1}, X_{2}}\left(x_{1}, x_{2}\right)+H_{X_{1}, X_{2}}\left(t_{1}, x_{2}\right) .
$$

Considering $x_{2}>0$ as a constant, the last expression is a Cauchy functional equation in $x_{1}$ and $t_{1}$ with continuous solution given by $H_{X_{1}, X_{2}}\left(x_{1}, x_{2}\right)=x_{1} c_{2}\left(x_{2}\right)$, for some continuous function $c_{2}\left(x_{2}\right)$. Analogously, let $t_{1}=0$ and $x_{2}>0$ in (7.20) to obtain $H_{X_{1}, X_{2}}\left(x_{1}, x_{2}\right)=x_{2} c_{1}\left(x_{1}\right)$, for some continuous function $c_{1}\left(x_{1}\right)$. Comparing the two solutions we conclude that $\frac{c_{1}\left(x_{1}\right)}{x_{1}}=\frac{c_{2}\left(x_{2}\right)}{x_{2}}=c$ for $x_{1}, x_{2}>0$, i.e., $c_{i}\left(x_{i}\right)=c x_{i}$, for some real constant $c, i=1,2$. The cases $x_{1}=0$ or $x_{2}=0$ 
are addressed in Remark 7.6.1. Therefore we have $H_{X_{1}, X_{2}}\left(x_{1}, x_{2}\right)=c x_{1} x_{2}$, what concludes the proof.

Example 7.6.3. If $c=0$ we obtain $H_{X_{1}, X_{2}}\left(x_{1}, x_{2}\right)=0$ for all $x_{1}, x_{2} \geq 0$, which is the trivial solution of the functional equation (7.20) and corresponds to $X_{1}$ being independent of $X_{2}$. Additionally, if $X_{1}$ and $X_{2}$ are exponentially distributed, we recover the result in Theorem 2.1 in Marshall and Olkin (1967).

Recall that Gumbel's Type I bivariate exponential distribution (5.3) has dependence function $H_{X_{1}, X_{2}}\left(x_{1}, x_{2}\right)=-\theta \lambda_{1} \lambda_{2} x_{1} x_{2}=c x_{1} x_{2}$. Therefore, (5.3) satisfies this stronger version of S-BLMP.

In fact, it is not the only distribution with such a property. For example, consider $\left(Y_{1}, Y_{2}\right)$ distributed according with (5.3) and the nonnegative bivariate random vector $\left(Z_{1}, Z_{2}\right)$ composed by independent absolutely continuous marginals. Both distributions possess the stronger version of S-BLMP. Since the dependence function $H_{Z_{1}, Z_{2}}\left(x_{1}, x_{2}\right)=0$ for all $x_{1}, x_{2}$, we conclude that $S_{Y_{1}, Y_{2}}\left(x_{1}, x_{2}\right) S_{Z_{1}, Z_{2}}\left(x_{1}, x_{2}\right)$ also possess the the property. In fact, closure properties similar with (S1) to (S4) in Theorem 7.4.1 hold true for this stronger version of S-BLMP.

Remark 7.6.4. In spite of the support of (5.3) be $\mathbf{R}_{+}^{2}$, the result obtained in Theorem 7.6.2 is also valid for survival functions with support in $\mathbf{R}^{2}$. In fact, consider the trivial solution of the functional equation (7.20), $H_{X_{1}, X_{2}}\left(x_{1}, x_{2}\right)=0$ for all $x_{1}, x_{1} \in \mathbf{R}$, and two independent marginals $X_{1}$ and $X_{2}$ with support in $\mathbf{R}$, for instance, two independent standard normal distributions. Then the support of $S_{X_{1}, X_{2}}\left(x_{1}, x_{2}\right)$ is $\mathbf{R}^{2}$ and the stronger version of $S$-BLMP holds true.

Since copulas are widely used to describe the dependence between random variables, it is worthwhile to consider how this concept is related with the result obtained in Theorem 7.6.2, where it is required that $\mathbf{X}$ and $\mathbf{X}_{\left(t_{1}, t_{2}\right)}$ have the same dependence function, see condition (7.19). In the rest of this section we provide a comparison between the stronger version of S-BLMP and the preservation of copula structure under truncation.

Oakes (2005) investigated a similar property, but with the dependence structure between the random variables $X_{1}$ and $X_{2}$ specified by the corresponding survival copula. According with Oakes (2005), if $\mathbf{X}$ has absolutely continuous distribution, $\mathbf{X}$ and $\mathbf{X}_{\left(t_{1}, t_{2}\right)}$ will share the same survival copula if and only if the survival copula is the Clayton copula given by

$$
C(u, v ; \delta)=\left(u^{-\delta}+v^{-\delta}-1\right)^{-\frac{1}{\delta}}, 0<\delta<\infty,
$$

see family B4 in Joe (1997).

Our result goes in a different direction, since we consider the original dependence function $H_{X_{1}, X_{2}}\left(x_{1}, x_{2}\right)$ while Oakes (2005) works with bivariate copulas, where $H_{X_{1}, X_{2}}\left(x_{1}, x_{2}\right)$ is transformed from the support of the original distribution to $[0,1]^{2}$, the support of bivariate copulas.

Paralleling the two approaches, notice that if one considers that a bivariate copula expresses the dependence between two random variables, then by just "plugging" univariate distributions into the copula, several bivariate distributions with same dependence structure can be obtained. Alternatively, considering the function $H_{X_{1}, X_{2}}\left(x_{1}, x_{2}\right)$ expresses the dependence, one has to rely on closure properties to generate other distributions with the same dependence function.

Interesting to notice that in both results unique solutions are obtained, but with different assumptions: Oakes (2005) requires absolutely continuity of $S_{X_{1}, X_{2}}\left(x_{1}, x_{2}\right)$ while we require the 
continuity of $H_{X_{1}, X_{2}}\left(x_{1}, x_{2}\right)$.

When $X_{1}$ and $X_{2}$ are independent random variables, the two results coincide. In fact, under independence, $\mathbf{X}$ and $\mathbf{X}_{\left(t_{1}, t_{2}\right)}$ have the same dependence function $H_{\mathbf{X}}\left(x_{1}, x_{2}\right)=H_{\mathbf{X}_{\left(t_{1}, t_{2}\right)}}\left(x_{1}, x_{2}\right)=0$, which can be obtained from Theorem 7.6.2 letting $c=0$, and also have the same survival copula, i.e., the independence copula $\bar{C}(u, v)=u v$, which can be obtained from Clayton copula as a limiting case, letting $\delta \rightarrow 0$ in (7.21).

Meanwhile, the result obtained in Theorem 7.6.2 does not hold true for any marginal distributions. In fact, $S_{X_{1}, X_{2}}\left(x_{1}, x_{2}\right)=\exp \left\{-x_{1}^{2}-x_{2}^{2}-c x_{1} x_{2}\right\}, x_{1}, x_{2} \geq 0, c \neq 0$, is not a proper survival function, since $\frac{\partial}{\partial x_{1} \partial x_{2}} S_{X_{1}, X_{2}}\left(x_{1}, x_{2}\right)$ is not necessarily nonnegative for all $x_{1}, x_{2} \geq 0$. Thus, the result is not a copula property and, as such, is not expressed in copula terms as in Oakes (2005).

\subsection{Concluding remarks}

In this chapter we introduced the Sibuya BLMP (S-BLMP) via relation (6.5), which covers a huge class of nonnegative bivariate continuous distributions $S_{X_{1}, X_{2}}\left(x_{1}, x_{2}\right)$ such that the dependence function $H_{X_{1}, X_{2}}\left(x_{1}, x_{2}\right)$, defined by relation (3.4), is shared with the survival function of the corresponding residual lifetime vector $\mathbf{X}_{t}$ for all $t>0$.

The S-BLMP is a weaker version of both BLMP (which preserves the joint survival functions of $\left(X_{1}, X_{2}\right)$ and $\left.\mathbf{X}_{t}\right)$ and LS-BLMP, introduced in Chapter 6. Therefore, the set of bivariate distributions possessing S-BLMP is very rich.

It happens that S-BLMP is equivalent to the class $\mathcal{L}(\mathbf{x} ; \mathbf{A})$ defined by relation (7.6), where the functions $A_{1}\left(x_{1}\right)$ and $A_{2}\left(x_{2}\right)$ are continuous and integrable. This equivalence is established by characterization theorems 7.3.4, 7.3.6 and 7.3.8. Additional characterizations in terms of functional equations have been obtained as well, see Lemma 7.2.2 and Lemma 7.3.3. The corresponding relations are displayed in Figure 7.1.

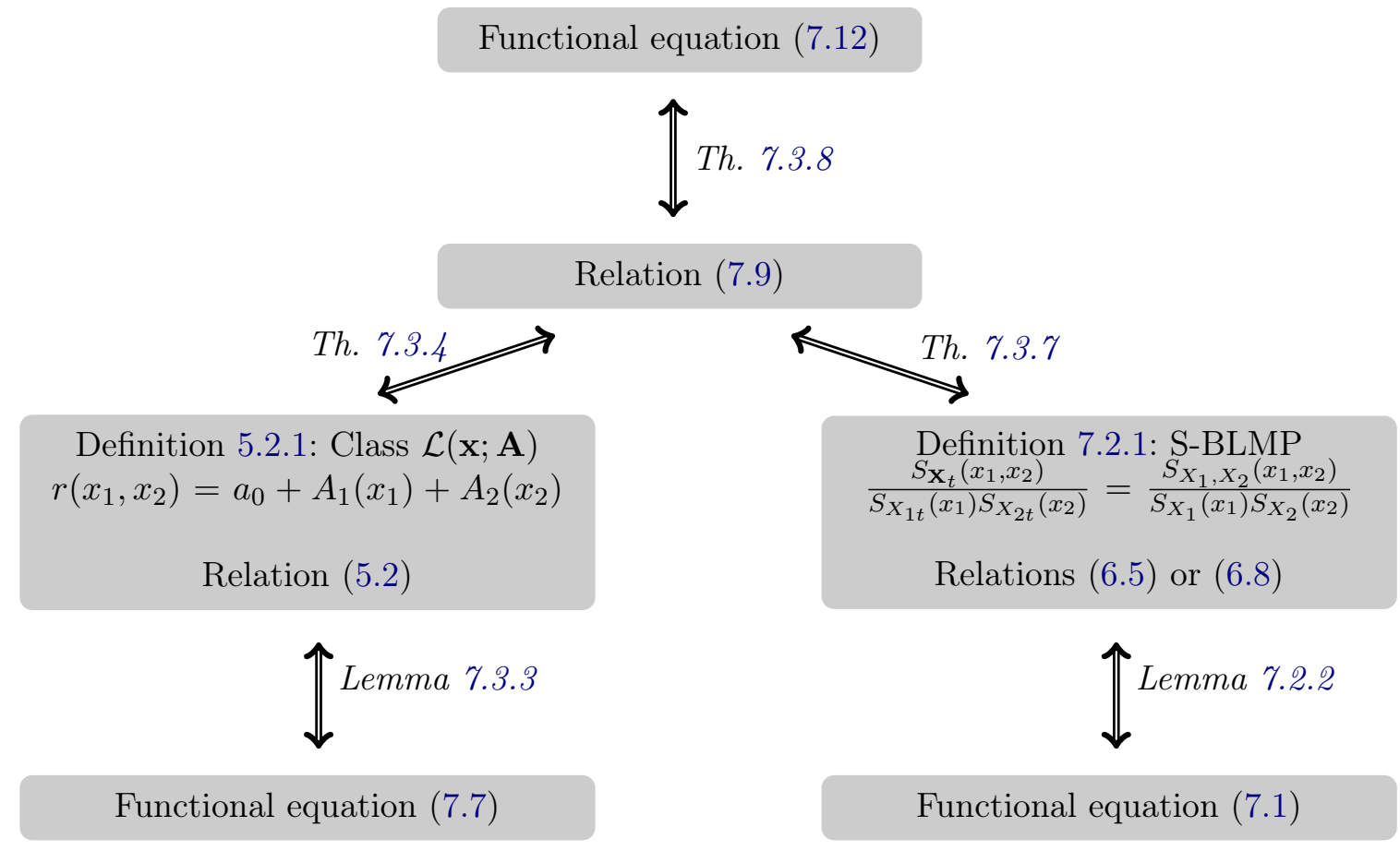

Figure 7.1: Equivalence relations for $S-B L M P$ and class $\mathcal{L}(\mathbf{x} ; \mathbf{A})$. 
Observe that the functional equation (7.12) is identical with (7.4), which was used to motivate the additive representation (7.6), used to define the class $\mathcal{L}(\mathbf{x} ; \mathbf{A})$. It should be underlined that expression (7.9) for the joint survival function $S_{X_{1}, X_{2}}\left(x_{1}, x_{2}\right)$ is, in fact, the solution of the functional equation (7.12).

A stochastic representation of the class $\mathcal{L}(\mathbf{x} ; \mathbf{A})$ was specified in Theorem 7.3.11 under the additional assumption of strictly increasing functions $A_{i}\left(x_{i}\right)$ in relation (7.6). The members of $\mathcal{L}(\mathbf{x} ; \mathbf{A})$ can be absolutely continuous or, additionally, exhibit a singular component. In Theorem 7.3.15 we obtained a simple condition that characterizes the absolutely continuous survival functions that belong to the extended class.

Based on characterization results and closure properties of S-BLMP we suggested in Section 7.4 two algorithms to generate distributions possessing S-BLMP.

It can be seen in Figure 7.2 the relation among the three lack-of-memory concepts so far discussed (classical BLMP, LS-BLMP introduced in Chapter 6 and S-BLMP). One will find in the corresponding intersection areas the position of many discussed examples, including Gumbel's type bivariate distributions, specified by (5.3) and (5.23), GMO and EMO models. In particular, GMO models possess S-BLMP if and only if $T_{3}$ is exponentially distributed in stochastic representation (2.9), see Lemma 7.2.7.

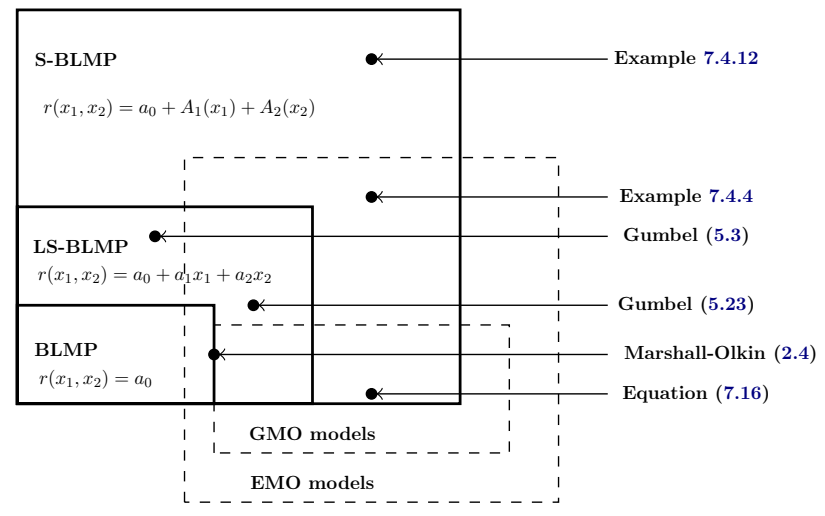

Figure 7.2: The three BLMP concepts and corresponding examples of distributions

We considered a stronger form of the S-BLMP and, under the assumption of continuity of the dependence function, obtained a unique solution for $H_{X_{1}, X_{2}}\left(x_{1}, x_{2}\right)$. This result is compared and contrasted with Oakes (2005) that, in a similar context, but considering the survival copula represents the dependence between two random variables, obtained as unique solution the Clayton copula.

The characterization results and established relations between well-known and new classes of bivariate distributions obtained in this chapter will help to profound and complement the BLMP knowledge by considering "non-aging" dependence function $H_{X_{1}, X_{2}}\left(x_{1}, x_{2}\right)$ as a base.

Further research on this topic include inference procedures, appropriate fields of application of S-BLMP distributions and multivariate extension of the discussed results. 


\section{Chapter 8}

\section{Conclusions}

In this last chapter, in Section 8.1 we summarize the main results obtained in the thesis. Moreover, since during the course of our work we identified open problems and possible extensions of our results, in Section 8.2 we suggest potential topics for future research. Finally, in Section 8.3 we report talks, technical reports and submitted papers based on the material presented in the previous chapters.

\subsection{Concluding remarks}

The classical Marshall-Olkin bivariate exponential distribution is relevant not only for its wide range of applications, such as Reliability, Survival Analysis, Finance and Life Insurance, but also for extending the univariate lack-of-memory property to the bivariate and multivariate settings. Among recent theoretical and applied advances in Marshall-Olkin exponential distribution we can mention Mai and Scherer (2010), Mai (2010), Mai and Scherer (2011), Lin and Li (2012), Bernhart et al. (2013) and Baglioni and Cherubini (2013).

In this thesis, our starting point was the generalized Marshall-Olkin distribution of Li and Pellerey (2011), where we introduced dependence among the individual shocks obtaining the extended Marshall-Olkin model. Corresponding survival copula representation, probabilistic and reliability properties as well as extreme value result were provided. Applying a similar approach, we introduced dependence among the individual shocks in the dual generalized Marshall-Olkin distribution of Fang and Li (2013) obtaining the dual extended Marshall-Olkin model. Related copula representation as well as probabilistic and reliability properties were discussed.

Given that the Marshall-Olkin bivariate exponential distribution is characterized by the bivariate lack-of-memory property, in a related topic of research we considered a weaker version of this property, where the lack-of-memory holds only for the dependence function $H_{X_{1}, X_{2}}\left(x_{1}, x_{2}\right)$. Initially we introduced a new class of distributions such that the sum of the components of the hazard gradient vector is a linear function of both arguments $x_{1}$ and $x_{2}$. Then we showed all bivariate distributions that belong to this class are characterized by a memoryless dependence function along with a specific aging behaviour on the marginals. Geometric interpretations, characterization results building procedures, survival copula relation and reliability properties were discussed.

Then we introduced a more general class of bivariate distributions, such that the sum of the components of the hazard gradient vector is decomposable into a sum of two functions, one that only depends on $x_{1}$ and another that only depends on $x_{2}$. All bivariate distributions that belong 
to this class are characterized by a memoryless dependence function, i.e., this new class is obtained from the previously introduced class relaxing the aging behaviour condition on the marginals. Stochastic representation, characterization of the absolutely continuous members and a general building procedure were presented.

Finally, we offered a stronger version of the bivariate lack-of-memory property previously introduced, obtaining a result comparable to Oakes (2005), where copulas are considered to represent the dependence between two random variables.

\subsection{Future research}

The topics addressed in this thesis open some potential subjects for future research which are presented at the end of each chapter. In this section we summarize them. Concerning the extended Marshall-Olkin model introduced in Chapter 3, one possibility consists in obtaining an absolutely continuous version of the EMO distribution, following an approach similar to Block and Basu (1974). Such a model should be suitable to analyse data without ties. Estimation techniques for extended MO models, with or without censoring, remain to be developed as well as the multivariate version. Similar research, related to the dual extended Marshall-Olkin model introduced in Chapter 4, can also be performed.

In Chapter 5 we introduced the class $\mathcal{L}(\mathbf{x} ; \mathbf{a})$ composed by nonnegative bivariate continuous distributions such that the sum of the components of hazard gradient vector is a linear function of both arguments $x_{1}$ and $x_{2}$. A parallel methodology remains to be elaborated in bivariate and multivariate discrete settings. Our approach did not cover the censoring data case as well. The inference procedures for the elements of $\mathcal{L}(\mathbf{x} ; \mathbf{a})$ and applications for real data sets are also object of future research. Difficulties would come in the case of continuous distributions with singular part and one possible solution, even in the case of censoring, is to apply the Kaplan-Meier estimate of bivariate survival functions, following Dabrowska (1988). In fact, the results in the cited paper can serve as a key-stone approach to extend the class $\mathcal{L}(\mathbf{x} ; \mathbf{a})$ of continuous bivariate distributions to a larger class having arbitrary marginal distributions.

Another potential topic for future research is based on the recent paper by Nair and Sankaran (2013). The authors construct a bivariate absolutely continuous and symmetric survival function of the form $S_{X_{1}, X_{2}}\left(x_{1}, x_{2}\right)=\exp \left\{-g\left(x_{1}, x_{2}\right)\right\}$ where the continuous function $g\left(x_{1}, x_{2}\right)$ is defined by functional equation $g\left(x_{1}, x_{2}\right)=H_{Y}^{-1}\left(H_{Y}\left(x_{1}\right)+H_{Y}\left(x_{2}\right)\right)$. Here, $H_{Y}(x)$ is the cumulative failure rate function of the nonnegative random variable $Y$ which satisfies the relation $\mathbb{P}\left(Y>g\left(x_{1}, x_{2}\right) \mid Y>\right.$ $\left.x_{1}\right)=\mathbb{P}\left(Y>x_{2}\right)$, see Proposition 2.1 in Nair and Sankaran (2013). The usual univariate LMP is a particular case substituting $g\left(x_{1}, x_{2}\right)=x_{1}+x_{2}$ in the last equation. Our suggestion is to define a new kind of BLMP by the condition

$$
\mathbb{P}\left(X_{1}>\gamma_{1}\left(x_{1}, t\right), X_{2}>\gamma_{1}\left(x_{2}, t\right) \mid X_{1}>\gamma_{2}(t), X_{2}>\gamma_{2}(t)\right) \text { is independent of } t,
$$

under corresponding compatibility constraints on continuous functions $\gamma_{1}(x, t)$ and $\gamma_{2}(t)$. It is clear that the classical bivariate lack-of-memory property, see relation (2.7), is the result of letting $\gamma_{1}(x, t)=x+t$ and $\gamma_{2}(t)=t$ in the foregoing relation.

In Chapter 6 we defined the concept of LS-BLMP and showed it can be used to characterize the class $\mathcal{L}(\mathbf{x} ; \mathbf{a})$. In Chapter 7 we extended the results obtained in Chapter 6 . Similar approaches 
can be followed as the discrete and multivariate versions of the class $\mathcal{L}(\mathbf{x} ; \mathbf{a})$ are developed.

\subsection{Talks, technical reports and submitted papers}

Earlier versions of the results obtained in this thesis have been reported in several opportunities, as shown in Table 8.1.

Table 8.1: Conferences, workshops and talks

\begin{tabular}{|c|c|}
\hline & Conferences, Workshops, Talks \\
\hline $\begin{array}{l}\text { On the IFR aging of biv } \\
\text { butions under binary as }\end{array}$ & $\begin{array}{l}\text { Probability Theory and its Applications - In } \\
\text { Commemoration of the Centennial of B.V. } \\
\text { Gnedenko (Moscow, June 2012) }\end{array}$ \\
\hline $\begin{array}{l}\text { Extended Marshall-Olkin Model and Finance } \\
\text { Applications }\end{array}$ & $\begin{array}{l}\text { Talk held at IMPA (Rio de Janeiro, August } \\
\text { 2012) }\end{array}$ \\
\hline $\begin{array}{l}\text { Extended Marshall-Olkin Model and Finance } \\
\text { Applications }\end{array}$ & $\begin{array}{l}1^{\text {st }} \text { European Actuarial Journal (EAJ) Con- } \\
\text { ference (Lausanne, September 2012) }\end{array}$ \\
\hline $\begin{array}{l}\text { Extended Marshall-Olkin Model and Finance } \\
\text { Applications }\end{array}$ & $\begin{array}{l}\text { Talk at Technische Universität München } \\
\text { (München, September 2012) }\end{array}$ \\
\hline $\begin{array}{l}\text { Marshall-Olkin Model and Finance } \\
\text { ons }\end{array}$ & $\begin{array}{l}\text { Talk at Technical University of Innsbruck } \\
\text { (Innsbruck, September 2012) }\end{array}$ \\
\hline $\begin{array}{l}\text { Marshall-Olkin Model and Finance } \\
\text { ons }\end{array}$ & $\begin{array}{l}\text { Workshop on Stochastic Modeling at Piraeus } \\
\text { University (Athens, October 2012) }\end{array}$ \\
\hline $\begin{array}{l}\text { Marshall-Olkin Model and Finance } \\
\text { ons }\end{array}$ & $\begin{array}{l}\text { University of Economics } \\
012 \text { ) }\end{array}$ \\
\hline $\begin{array}{l}\text { d Marshall-Olkin Model and Finance } \\
\text { ions }\end{array}$ & $\begin{array}{l}\text { Satellite Copula Work } \\
\text { CAMP (Campinas, M }\end{array}$ \\
\hline $\begin{array}{l}\text { Properties of the Extended Marshall-Olkin } \\
\text { Model }\end{array}$ & $\begin{array}{l}\text { Satellite Copula Worksh } \\
\text { CAMP (Campinas, Mar }\end{array}$ \\
\hline on bivariate extreme value copulas & $\begin{array}{l}\text { Sixth Brazilian Conference on Statistical } \\
\text { Modelling in Insurance and Finance (Mare- } \\
\text { sias, March 2013) }\end{array}$ \\
\hline $\begin{array}{l}\text { ate distribut } \\
\text { gradient cc }\end{array}$ & $\begin{array}{l}\text { Talk held at UFSCAR and ICMC-USP (São } \\
\text { Carlos, August 2013) }\end{array}$ \\
\hline la function & $\begin{array}{l}\text { Risk Management Reloaded - Technische } \\
\text { Universität München (München, September } \\
\text { 2013) }\end{array}$ \\
\hline $\begin{array}{l}\text { Continuous bivariate distributions with linear } \\
\text { sum of the hazard gradient components and } \\
\text { actuarial applications }\end{array}$ & $\begin{array}{l}\text { Financial and Actuarial Mathematics at } \\
\text { Technische Universität Wien (Vienna, } \\
\text { September 2013) }\end{array}$ \\
\hline $\begin{array}{l}\text { Extended Marshall-Olkin Model and its dual } \\
\text { version }\end{array}$ & $\begin{array}{l}\text { Marshall-Olkin Distributions: Advances in } \\
\text { Theory and Applications (Bologne, October } \\
\text { 2013) }\end{array}$ \\
\hline $\begin{array}{l}\text { Extended Marshall-Olkin Model and Appli- } \\
\text { cations }\end{array}$ & $\begin{array}{l}\text { Marshall-Olkin Distributions: Advances in } \\
\text { Theory and Applications (Bologne, October } \\
\text { 2013) }\end{array}$ \\
\hline $\begin{array}{l}\text { A new notion of bivariate lack-of-memory } \\
\text { property }\end{array}$ & $\begin{array}{l}3^{r d} \text { Workshop in Survival Analysis and Ap- } \\
\text { plications (Campinas, November 2013) }\end{array}$ \\
\hline
\end{tabular}


Four Technical Reports were elaborated at the Institute of Mathematics and Statistics of the University of São Paulo, according with Table 8.2:

Table 8.2: Technical Reports at Institute of Mathematics and Statistics (USP)

\begin{tabular}{|l|l|}
\hline \multicolumn{1}{|c|}{ Title } & \multicolumn{1}{|c|}{ Number } \\
\hline Extended Marshall-Olkin model and applications & RT-MAE 2012-06, 13p. \\
\hline $\begin{array}{l}\text { Continuous bivariate distributions with linear sum of the hazard } \\
\text { gradient components }\end{array}$ & RT-MAE 2013-05, 32p. \\
\hline Sibuya-type Bivariate Lack of Memory Property & RT-MAE 2014-01, 23p. \\
\hline Extreme value properties of the extended Marshall-Olkin model & RT-MAE 2014-02, 12p. \\
\hline
\end{tabular}

Six papers were submitted for publication:

1. Extended Marshall-Olkin Model and Applications;

2. Dual Extended Marshall-Olkin Model and Applications;

3. Continuous Bivariate Distributions with Linear Sum of the Hazard Gradient Components;

4. Linear Sibuya-type Bivariate Lack of Memory Property;

5. Extreme value properties of the Extended Marshall-Olkin model;

6. Sibuya-type bivariate lack of memory property.

Finally, there are two invitations to write chapters in Proceedings of Springer Verlag covering part of the material presented in this thesis. 


\section{Bibliography}

Aczèl (1966) J. Aczèl. Lectures on Functional Equations and Their Applications. Academic Press. Cited on page(s) 10

Al-Zahrani and Stoyanov (2008) B. Al-Zahrani and J. Stoyanov. On some properties of live distributions with increasing elasticity and log-concavity. Applied Mathematical Sciences, 2:23492361. Cited on page(s) 39

An (1998) M.Y. An. Logconcavity versus logconvexity: a complete characterization. Journal of Economic Theory, 80:350-369. Cited on page(s) 39, 40

Arnold (1968) B. Arnold. Parameter estimation for a multivariate exponential distribution. Journal of the American Statistical Association, 63:848-852. Cited on page(s) 9

Arnold and Strauss (1988) B.C. Arnold and D. Strauss. Pseudolikelihood estimation. Sankhya: The Indian Journal of Statistics, 53:233-243. Series B. Cited on page(s) 5

Asadi (1999) M. Asadi. Multivariate distributions characterized by a relationship between mean residual life and hazard gradient. Metrika, 49:121-126. Cited on page(s) 98

Asha and John (2009) G. Asha and R.C. John. Characterizations using the generalized reversed lack of memory property. Statistics \& Probability Letters, 79:1480-1487. Cited on page(s) 13

Azzalini (1985) A. Azzalini. A class of distributions which include the normal ones. Scandinavian Journal of Statistics, 12:172-178. Cited on page(s) 40

Azzalini and Dalla Valle (1996) A. Azzalini and A. Dalla Valle. The multivariate skew-normal distribution. Biometrika, 83:715-726. Cited on page(s) 40

Baglioni and Cherubini (2013) A. Baglioni and U. Cherubini. Within and between systemic country risk. theory and evidence from the sovereign crisis in Europe. Journal of Economic Dynamics and Control, 37:1581-1597. Cited on page(s) 143

Bagnoli and Bergstrom (2005) M. Bagnoli and T. Bergstrom. Log-concave probability and applications. Economic Theory, 26:445-469. Cited on page(s) 39, 42

Balakrishnan and Basu (1995) N. Balakrishnan and A.P. Basu. The Exponential Distribution: Theory, Methods and Applications. Gordon \& Breach Science Publishers, Newark, N.J. Cited on page(s) 1

Balakrishnan and Lai (2009) N. Balakrishnan and C.D. Lai. Continuous Bivariate Distributions. Springer, Second ed. Cited on page(s) 5, 6, 9, 24, 29, 30, 41, 50, 73, 103

Barlow and Mendel (1992) R. Barlow and M. Mendel. De-Finetti type representations for life distributions. Journal of the American Statistical Association, 87:1116-1122. Cited on page(s) 78

Barlow and Proschan (1981) R.E. Barlow and F. Proschan. Statistical Theory of Reliability and Life Testing. Silver Spring. Cited on page(s) 17, 19, 37, 40, 62, 75 
Barlow et al. (1963) R.E. Barlow, A.W. Marshall and F. Proschan. Properties of probability distributions with monotone hazard rate. The Annals of Mathematical Statistics, 34:375-389. Cited on page(s) 59

Barnett (1976) V. Barnett. The ordering of multivariate data (with discussion). Journal of the Royal Statistical Society, 139:318-354. Cited on page(s) 43

Bassan and Spizzichino (2005) B. Bassan and F. Spizzichino. Relations among univariate aging, bivariate aging and dependence for exchangeable lifetimes. Journal of Multivariate Analysis, 93: 313-339. Cited on page(s) 26

Basu (1971) A.P. Basu. Bivariate failure rate. Journal of American Statistical Association, 66: 103-104. Cited on page(s) 17, 40, 75, 99

Basu (1988) A.P. Basu. Multivariate exponential distributions and their applications in reliability. Handbook of Statistics, 7:467-477. Cited on page(s) 5

Beirlant et al. (2005) J. Beirlant, Y. Goegebeur, J. Segers and J. Teugels. Statistics of Extremes: Theory and Application. John Wiley \& Sons. Cited on page(s) 42, 44, 45

Bemis et al. (1972) B. Bemis, L.J. Bain and J.J. Higgins. Estimation and hypothesis testing for the parameters of a bivariate exponential distribution. Journal of the American Statistical Association, 67:927-929. Cited on page(s) 9, 83

Bernhart et al. (2013) G. Bernhart, M.E. Anel, J. Mai and M. Scherer. Default models based on scale mixtures of Marshall-Olkin copulas: properties and applications. Metrika, 76:179-203. Cited on page(s) 143

Bismi (2005) G. Bismi. Bivariate Burr distributions. Master Thesis, Cochin University of Science and Technology, India. Cited on page(s) 59

Block (1977) H.W. Block. A characterization of a bivariate exponential distribution. The Annals of Statistics, 5:808-812. Cited on page(s) 8

Block and Basu (1974) H.W. Block and A.P. Basu. A continuous bivariate exponential extension. Journal of the American Statistical Association, 69:1031-1037. Cited on page(s) xvii, 8, 27, 36, 52, $53,54,55,73,108,144$

Brindley and Thompson (1972) E.C. Brindley and W.A. Thompson. Dependence and aging aspects of multivariate survival. Journal of the American Statistical Association, 67:822-830. Cited on page(s) 40, 114

Charpentier (2003) A. Charpentier. Tail distribution and dependence measures. 2003. Cited on page(s) 137

Charpentier and Juri (2006) A. Charpentier and Alessandro Juri. Limiting dependence structures for tail events, with applications to credit derivatives. Journal of Applied Probability, 43: 563-586. Cited on page(s) 136, 137

Chukova and Dimitrov (1992) S. Chukova and B. Dimitrov. On distributions having the almostlack-of-memory property. Journal of Applied Probability, 29:691-698. Cited on page(s) 13

Dabrowska (1988) D. Dabrowska. Kaplan-Meier estimate on the plane. The Annals of Statistics, 16:1475-1489. Cited on page(s) 99, 100, 101, 144

Dimitrov and Kolev (2013) B. Dimitrov and N. Kolev. The BALM copula. International Journal of Stochastic Analysis, 2013:1-6. Cited on page(s) 13 
Dimitrov et al. (1994) B. Dimitrov, S. Chukova and Z. Khalil. Approximation, Probability and Related Fields, chapter Bivariate probability distributions similar to exponential, pages 167-178. Springer. Cited on page(s) 13

Fang and Li (2013) R. Fang and X Li. A note on bivariate dual generalized Marshall-Olkin distributions with applications. Probability in the Engineering and Informational Sciences, 27: 367-374. Cited on page(s) 2, 10, 57, 61, 62, 64, 67, 68, 71, 143

Finkelstein (2003) M. Finkelstein. On one class of bivariate distributions. Statistics \& Probability Letters, 65:1-6. Cited on page(s) 17

Finkelstein and Esaulova (2005) M.S. Finkelstein and V. Esaulova. On the weak IFR aging of bivariate lifetime distributions. Appl. Stochastic Models Bus. Ind., 21:265-272. Cited on page(s) 37, 38

Freund (1961) E.J. Freund. A bivariate extension of the exponential distribution. Journal of American Statistical Association, 56:971-977. Cited on page(s) 5, 73

Friday and Patil (1977) D.S. Friday and G.P. Patil. The Theory and Applications of Reliability, volume 1, chapter A bivariate exponential model with applications to reliability and computer generation of random variables, pages 527-549. Academic Press, New York. Cited on page(s) 5, 73

Galambos (1978) J. Galambos. The Asymptotic Theory of Extreme Order Statistics. John Wiley \& Sons. Cited on page(s) 42, 43, 45

Galambos and Kotz (1978) J. Galambos and S. Kotz. Characterizations of probability distributions. Springer, Berlin. Cited on page(s) 1, 5

Ghorpade and Limaye (2010) S.R. Ghorpade and B.V. Limaye. A Course in Multivariate Calculus and Analysis. Springer. Cited on page(s) 39

Ghurye (1987) S.G. Ghurye. Some multivariate lifetime distributions. Advances in Applied Probability, 19:138-155. Cited on page(s) 13, 103, 108

Ghurye and Marshall (1984) S.G. Ghurye and A.W. Marshall. Shock processes with aftereffects and multivariate lack of memory. Journal of Applied Probability, 21:786-801. Cited on page(s) 8

Gourieroux and Monfort (2003) C. Gourieroux and A. Monfort. Age and term structure in duration models. Working paper. Cited on page(s) 138

Gradshteyn and Ryzhik (2007) I.S. Gradshteyn and I.M. Ryzhik. Tables of Integrals, Series and Products. Academic Press, Seventh ed. Cited on page(s) 83

Gumbel (1960) E.J. Gumbel. Bivariate exponential distributions. Journal of American Statistical Association, 55:698-707. Cited on page(s) xvii, 5, 52, 53, 54

Gupta and Gupta (1997) P.L. Gupta and R.C. Gupta. On the multivariate normal hazard. Journal of Multivariate Analysis, 62:64-73. Cited on page(s) 40

Gupta and Gupta (2001) P.L. Gupta and R.C. Gupta. Failure rate of the minimum and maximum of a multivariate normal distribution. Metrika, 53:39-49. Cited on page(s) 40

Gupta and Balakrishnan (2012) R. Gupta and N. Balakrishnan. Log-concavity and monotonicity of hazard and reversed hazard functions of univariate and multivariate skew normal distributions. Metrika, 75:181-191. Cited on page(s) 40

Gupta et al. (1998) R.C. Gupta, R.D. Gupta and P.L. Gupta. Modeling failure time data by Lehman alternatives. Communications in Statistics - Theory and Methods, 27:887-904. Cited on page(s) 64 
Harris (1970) R. Harris. A multivariate definition for increasing hazard rate distribution functions. Institute of Mathematical Statistics, 41:713-717. Cited on page(s) 40

Horrance (2005) W.C. Horrance. Some results on the multivariate truncated normal distribution. Journal of Multivariate Analysis, 94:209-221. Cited on page(s) 40

$\mathrm{Hu}$ and $\mathrm{Li} \mathrm{(2007)} \mathrm{T} \mathrm{Hu}$ and $\mathrm{Y} \mathrm{Li}$. Increasing failure rate and decreasing reversed hazard rate properties of the minimum and maximum of multivariate distributions with log-concave densities. Metrika, 65:325-330. Cited on page(s) 39, 40

Joe (1997) H. Joe. Multivariate Models and Dependence Concepts. Chapman \& Hall, London. Cited on page(s) 24, 31, 44, 47, 50, 64, 109, 139

John (2011) R.C. John. A study on the reversed lack of memory property and its generalizations. PhD Thesis, Cochin University of Science and Technology. Cited on page(s) 13

Johnson and Kotz (1975) N.L. Johnson and S. Kotz. A vector multivariate hazard rate. Journal of Multivariate Analysis, 5:53-66. Cited on page(s) 6, 13, 17, 40, 74, 81, 114

Karlis (2003) D. Karlis. ML estimation for multivariate shock models via an EM algorithm. Annals of the Institute of Statistical Mathematics, 55:817-830. Cited on page(s) 9

Keilson and Sumita (1982) J. Keilson and U. Sumita. Uniform stochastic ordering and related inequalities. The Canadian Journal of Statistics, 10:181-198. Cited on page(s) 59

Kotz et al. (2000) S. Kotz, N. Balakrishnan and N.L. Johnson. Continuous Multivariate Distributions, volume 1. John Wiley and Sons, New York, Second ed. Cited on page(s) 5, 6, 9

Kulkarni (2006) H.V. Kulkarni. Characterizations and modelling of multivariate lack of memory property. Metrika, 64:167-180. Cited on page(s) 8, 36, 73, 74, 77, 81, 82, 88, 92, 119, 131, 132, 133, 134,135

Kundu and Gupta (2010) D. Kundu and R.D. Gupta. A class of absolutely continuous bivariate distributions. Journal of Statistical Methodology, 7:464-477. Cited on page(s) 55

Lehmann (1966) E.L. Lehmann. Some concepts of dependence. The Annals of Mathematical Statistics, 37:1137-1153. Cited on page(s) 18, 61

Li (2008) H. Li. Duality of the multivariate distributions of Marshall-Olkin type and tail dependence. Communications in Statistics - Theory and Methods, 37:1721-1733. Cited on page(s) 64

Li and Pellerey (2011) X. Li and F. Pellerey. Generalized Marshall-Olkin distributions and related bivariate aging properties. Journal of Multivariate Analysis, 102:1399-1409. Cited on page(s) $10,15,19,24,26,57,71,78,94,120,122,143$

Lin and Li (2012) J. Lin and X. Li. Multivariate generalized Marshall-Olkin distributions and copulas. Methodology and Computing in Applied Probability. doi: 10.1007/s11009-012-9297-4. Cited on page(s) 143

Ma (1996) C. Ma. Multivariate survival functions characterized by constant product of mean remaining lives and hazard rates. Metrika, 44:71-83. Cited on page(s) 30, 41

Ma (2000) C. Ma. A note on the multivariate normal hazard. Journal of Multivariate Analysis, 73:282-283. Cited on page(s) 40

Mai (2010) J. Mai. Extendibility of Marshall-Olkin distributions via Lévy subordinators and an application to portfolio credit risk. PhD Thesis, Technische Universität München. Cited on page(s) 143 
Mai and Scherer (2010) J.F. Mai and M. Scherer. The Pickands representation of survival Marshall-Olkin copulas. Statistics \& Probability Letters, 80:357-360. Cited on page(s) 50, 143

Mai and Scherer (2011) J.F. Mai and M. Scherer. Reparameterizing Marshall-Olkin copulas with applications sampling. Journal of Statistical Computation and Simulation, 81:59-78. Cited on page(s) 143

Marshall (1975) A.W. Marshall. Some comments on the hazard gradient. Stochastic Processes and their Applications, 3:293-300. Cited on page(s) 74, 79

Marshall and Olkin (1967) A.W. Marshall and I. Olkin. A multivariate exponential distribution. Journal of American Statistical Association, 62:30-41. Cited on page(s) xvii, 1, 2, 5, 6, 7, 8, 10, 15, $52,53,73,86,88,136,138,139$

Marshall and Olkin (1995) A.W. Marshall and I. Olkin. Multivariate exponential and geometric distributions with limited memory. Journal of Multivariate Analysis, 53:110-125. Cited on page(s) 12

McNeil et al. (2005) A. McNeil, L. Frey and P. Embrechts. Quantitative Risk Management. Princeton University Press. Cited on page(s) 26, 44

Meintanis (2007) S.G. Meintanis. Test of fit for Marshall-Olkin distributions with applications. Journal of Statistical Planning and Inference, 137:3954-3963. Cited on page(s) xv, 9, 52, 55

Mulero and Pellerey (2010) J. Mulero and F. Pellerey. Bivariate aging properties under Archimedean dependence structures. Communications in Statistics: Theory and Methods, 39: 3108-3121. Cited on page(s) 26, 137

Muliere and Scarsini (1987) P. Muliere and M. Scarsini. Characterization of a Marshall-Olkin type class of distributions. Annals of the Institute of Statistical Mathematics, 39:429-441. Cited on page(s) $10,11,99$

Munkres (1991) J.R. Munkres. Analysis on Manifolds. Addison-Wesley Publishing Company. Cited on page(s) 75,76

Nair and Nair (1988a) K.R.M. Nair and N.U. Nair. On characterizing the bivariate exponential and geometric distributions. Annals of the Institute of Statistical Mathematics, 40:267-271. Cited on page(s) 6,12

Nair and Nair (1988b) N.U. Nair and V.K.R. Nair. A characterization of the bivariate exponential distribution. Biometrical Journal, 30:107-112. Cited on page(s) 6

Nair and Sankaran (2013) U. Nair and P. Sankaran. A family of bivariate exponential distributions and their copulas. Sankhya B. doi: 10.1007/s13571-013-0067-2. Cited on page(s) 78, 99, 144

Nair et al. (2004) U. Nair, G. Asha and P. Sankaran. On the covariance of residual lifes. Statistica, 64:573-585. Cited on page(s) 99

Navarro (2008) J. Navarro. Characterizations using the bivariate failure rate function. Statistics \& Probability Letters, 78:1349-1354. Cited on page(s) 75

Nelsen et al. (2001) R. Nelsen, J.J. Quesada-Molina, J.A. Rodríguez-Lallena and M. Úbeda Flores. Bounds on bivariate distribution functions with given margins and measures of association. Communications in Statistics - Theory and Methods, 30:1155-1162. Cited on page(s) 22

Nelsen (2006) R.B. Nelsen. An Introduction to Copulas. Springer, Second ed. Cited on page(s) 9, $19,21,22,23,43,48,63,68,109$ 
Oakes (2005) D. Oakes. On the preservation of copula structure under truncation. The Canadian Journal of Statistics, 33:465-468. Cited on page(s) 2, 139, 140, 141, 144

Obretenov (1985) A. Obretenov. Characterization of the multivariate Marshall-Olkin exponential distribution. Probability and Mathematical Statistics, 6:51-56. Cited on page(s) 8

Peña and Gupta (1990) A. Peña and A.K. Gupta. Bayes estimation for the Marshall-Olkin exponential distribution. Journal of the Royal Statistical Society, 52:379-389. Cited on page(s) 9

Pickands (1981) J. Pickands. Multivariate extreme value distributions. Bulletin of the International Statistical Institute, Proceedings of the 43rd Session, pages 859-878. Cited on page(s) 44

Proschan and Sullo (1976) F. Proschan and P. Sullo. Estimating the parameters of a multivariate exponential distribution. Journal of the American Statistical Association, 71:465-472. Cited on page(s) 9,73

Raja Rao et al. (1993) B. Raja Rao, C.V. Damaraju and J.M. Alhumound. Setting the clock back to zero property of a class of bivariate distributions. Communications in Statistics: Theory and Methods, 22:2067-2080. Cited on page(s) 6, 11

Roy (2002a) D. Roy. On bivariate lack of memory property and a new definition. Annals of the Institute of Statistical Mathematics, 54:404-410. Cited on page(s) 6, 13, 74

Roy (2002b) D. Roy. A characterization of model approach for generating bivariate life distributions using reversed hazard rates. Journal of The Japan Statistical Society, 32:239-245. Cited on page(s) 60

Roy (2004) D. Roy. A generalization of the lack of memory property and related characterization results. Communications in Statistics: Theory and Methods, 33:3145-3158. Cited on page(s) 6, 12, 98

Roy and Gupta (1996) D. Roy and R.P. Gupta. Bivariate extension of lomax and finite range distributions through characterization approach. Journal of Multivariate Analysis, 59:22-22. Cited on page(s) 12

Roy and Mukherjee (1989) D. Roy and S.P. Mukherjee. Characterizations of some bivariate life-distributions. Journal of Multivariate Analysis, 28:1-8. Cited on page(s) 8

Roy and Roy (2010) D. Roy and R. Roy. Characterizations of bivariate and multivariate life distributions based on reciprocal subtangent. Communications in Statistics - Theory and Methods, 39:158-169. Cited on page(s) 99

Sahoo and Kannappan (2011) P.K. Sahoo and P. Kannappan. Introduction to Functional Equations. CRC Press, Boca Raton. Cited on page(s) 36, 76, 107

Sankaran (1992) P.G. Sankaran. Characterization of Probability Distributions by Reliability Concepts. PhD Thesis, Cochin University, India. Cited on page(s) 12, 98

Sankaran and Gleeja (2006) P.G. Sankaran and V.L. Gleeja. On bivariate reversed hazard rates. Journal of The Japan Statistical Society, 36:213-224. Cited on page(s) 59, 60

Scott (1992) D. Scott. Multivariate Density Estimation: Theory, Practice and Visualization. Wiley-Interscience, New York. Cited on page(s) 101

Shaked and Shanthikumar (2007) M. Shaked and J.G. Shanthikumar. Stochastics Orders. Springer, New York, New York. Cited on page(s) 27, 67 
Shamseldin (1979) A.A. Shamseldin. Bayesian Parameter and Reliability Estimation for a Multivariate Exponential Distribution. PhD Thesis, University of California, Riverside, CA. Cited on page(s) 9

Shamseldin and Press (1984) A.A. Shamseldin and S.J. Press. Bayesian parameter and reliability estimation for a bivariate exponential distribution: parallel sampling. Journal of Econometrics, 24:363-378. Cited on page(s) 9

Shanbhag and Kotz (1987) D. Shanbhag and S. Kotz. Some new approaches to multivariate probability distributions. Journal of Multivariate Analysis, 22:189-211. Cited on page(s) 98

Sibuya (1960) M. Sibuya. Bivariate extreme statistics, I. Annals of the Institute of Statistical Mathematics, 11:195-210. Cited on page(s) 105

Singpurwalla (2006) N. Singpurwalla. Reliability and Risk: A Bayesian Perspective. Wiley, Chichester. Cited on page(s) 26, 32

Spiegelhalter et al. (2002) D.J. Spiegelhalter, N.G. Best, B.P. Carlin and A. Van Der Linde. Bayesian measures of model complexity and fit (with discussion). Journal of the Royal Statistical Society, 64:583-639. Cited on page(s) 53

Tawn (1988) J.A. Tawn. Bivariate extreme value theory: Models and estimation. Biometrika, 75: 397-415. Cited on page(s) 45, 50

Walther (2009) G. Walther. Inference and modeling with log-concave distributions. Statistical Science, 24:319-327. Cited on page(s) 39 